UC-NRLF

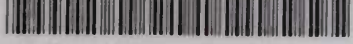

B 3 7b6 500 


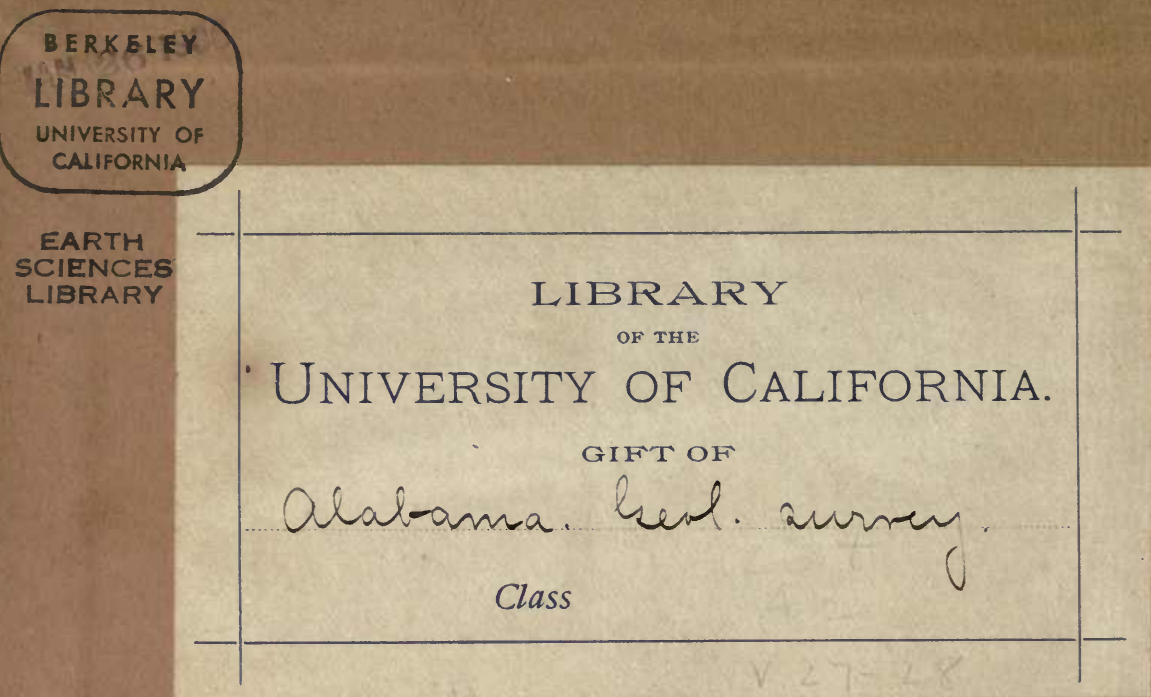






\title{
List no.27
}

\section{GEOLOGICAL SURVEY OF ALABAMA} EUGENE ALLEN SMITH, STATE GeOLOGIST

\section{BULLETIN No. 7.}

\author{
A PRELIMINARY REPORT
}

ON A PART OF THE

\section{Water Powers of Alabama} BY

B. M. HALL, CONAUT,TING ENGINEER, U. S. GEOL. SERVEY, For Gkorgia, Plotida, Tennegske and Mrgsigsippi.

1903 



\section{GEOLOGICAL SURVEY OF ALABAMA}

EUGENE ALLEN SMITH, State Geologist

\section{BULLETIN No. 7}

\section{A PRELIMINARY REPORT}

ON A PART OF THE

\section{WATER POWERs of Alabama}

BY

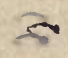

B. M. HALL,

- Congulting Engin aer, U. S. Geol. Survey,

for Geolegia, Florida, Tennessee and Mississippl.

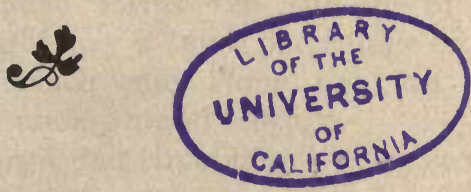

MONTGOMERY, ALA.:

THE BROWN PIINTING CO.. PRINTERS AND BINDERS.

1903. 

To His Excellency, William D. Jelks, Governor of Alabama:

Dear Sir: I have the honor to transmit herewith a preliminary report on the Water Powers of Alabama, by B. M. Hall, of the United States Geological Survey, Consulting Engineer, Hydrographic Division, for Alabama, Florida, Georgia, Tennessee, and Mississippi.

Our Alabama Geological Survey, in coöperation with the United States Geological Survey, has for a number of years been engaged in a systematic investigation of the Water Resources of the State. In this investigation we have naturally been less interested in that portion of the rainfall which passes back into the atmosphere by evaporation, than in those portions which, temporarily at least, become more or less incorporated with the materials forming our land surface, and which on that account may be considered as forming a part of our territory. And our investigation of this earth water, (to use a term to distinguish it from the atmospheric water), may appropriately be followed along two lines: It may be concerned, I, with that part of the water which, collecting in rivulets, creeks and rivers, flows on towards the sea by open channels, $i$. e., the "run-off"; or 2 , it may take into account that part which soaks into the ground, and reaches the water courses or the sea only after an underground passage of greater or less duration, $i . e$., the ground water or the "in-soak," if we may be allowed the use of such a word.

While the proportion of the rainfall which appears in the run-off of the streams varies between very wide limits, depending on the geological formations, the locality, etc., in Alabama on an average, about fifty per cent. of the rainfall is lost by evaporation and the remainder forms the run-off of the streams, and, curiously enough, only a small percentage of this run-off is supplied by the surface water alone, for most of it reaches the water courses by underground seepage.

In the course of this underground circulation the water may reach the surface from springs, from ordinary shallow and deep wells, and from artésian wells, and may be utilized for domestic and municipal water supply, and rarely, in Alabama at least, for irrigation and for power. 
The present writer has had charge of this branch of the investigation, and his report on the Artesian and other underground Water Systems of the State is now in manuscript, and practically ready for the printers.

Most of the material for this report has been collected by the Alabama Geological Survey.

The run-off, on the other hand, is utilized for transportation, for domestic and municipal water supply, and for power, and this branch of the subject has been in charge of Mr. Hall, who has for some years been employed by the United States Geological Survey in collecting records of the gage heights, and in making surveys and discharge measurements of the principal streams of Alabama (and adjacent States), from which the values of these streams for the various purposes above enumerated may be closely estimated. In the collection of these data, the Alabama Geological Survey has contributed to the extent of paying the observers of the gage heights at seven stations along Alabama streams, but with this exception and apart from the map, the present Report has been prepared without cost to the State of Alabama. We are also indebted to the United States Geological Survey for most of the illustrations which appear in the body of the Report, and these cuts, as well as most of the data from which this Report has been compiled by Mr. Hall, have been published in the Annual Reports of the Director of the National Geological Survey.

While the present report deals with only one of the many uses to which the run-off of our streams may be nut, viz., for the production of power, this is in many respects, - pecially in Alabama, the most important of these uses, for the great increase in the applications of electricity has of late turned attention to the utilization for its production, of water powers which have heretofore been $a^{\prime}$ lowed to run to waste, and there can be little doubt but that 'a comparatively short time, all the available water power of the State will be turned to account.

Very respectfully,

EUGENE A. SMITH,

University of Alabama, State Geologist

Dec. 1, 1902. 


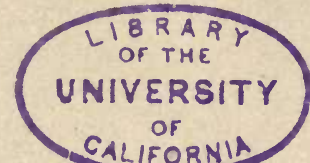

3

2 


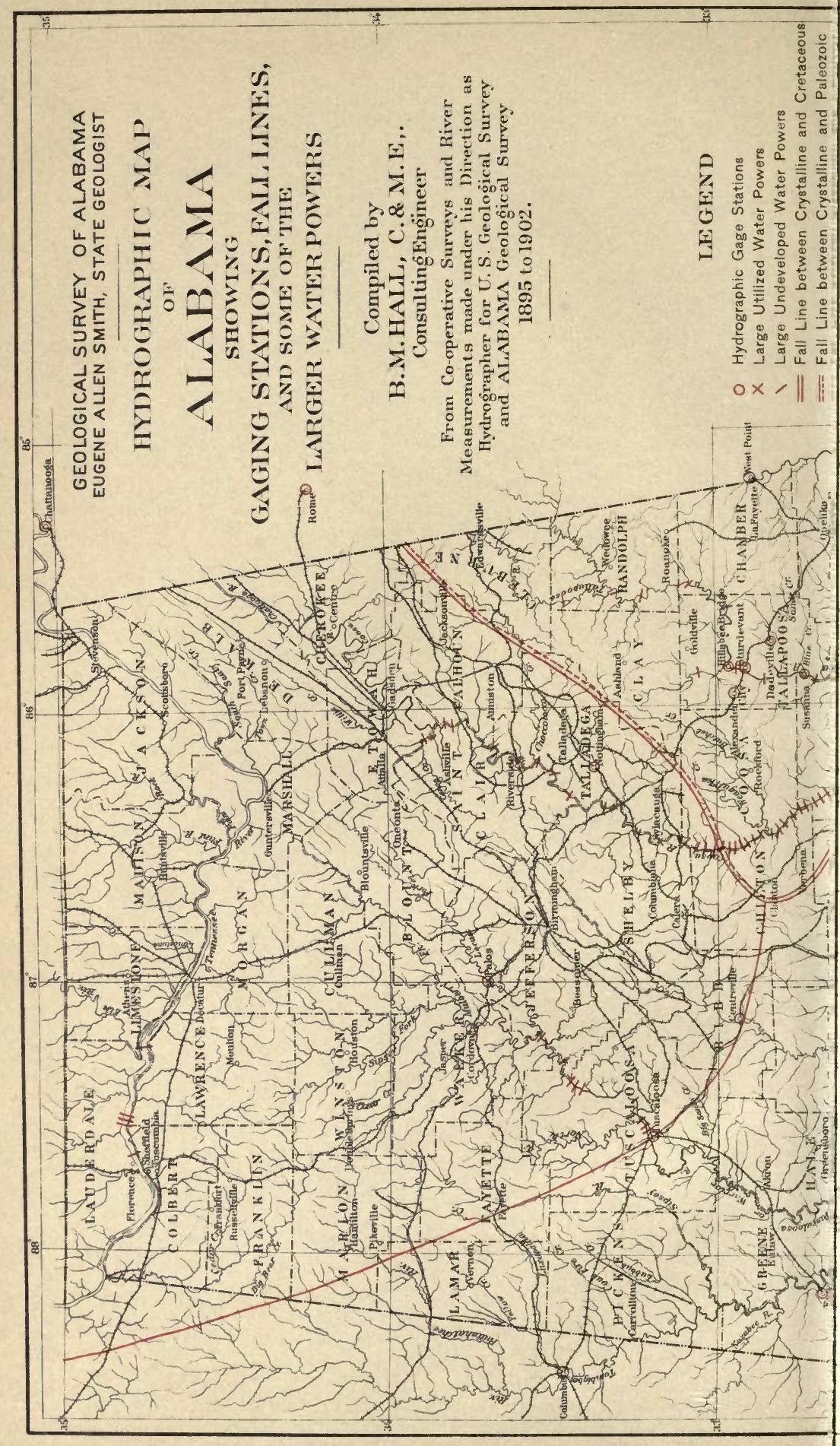




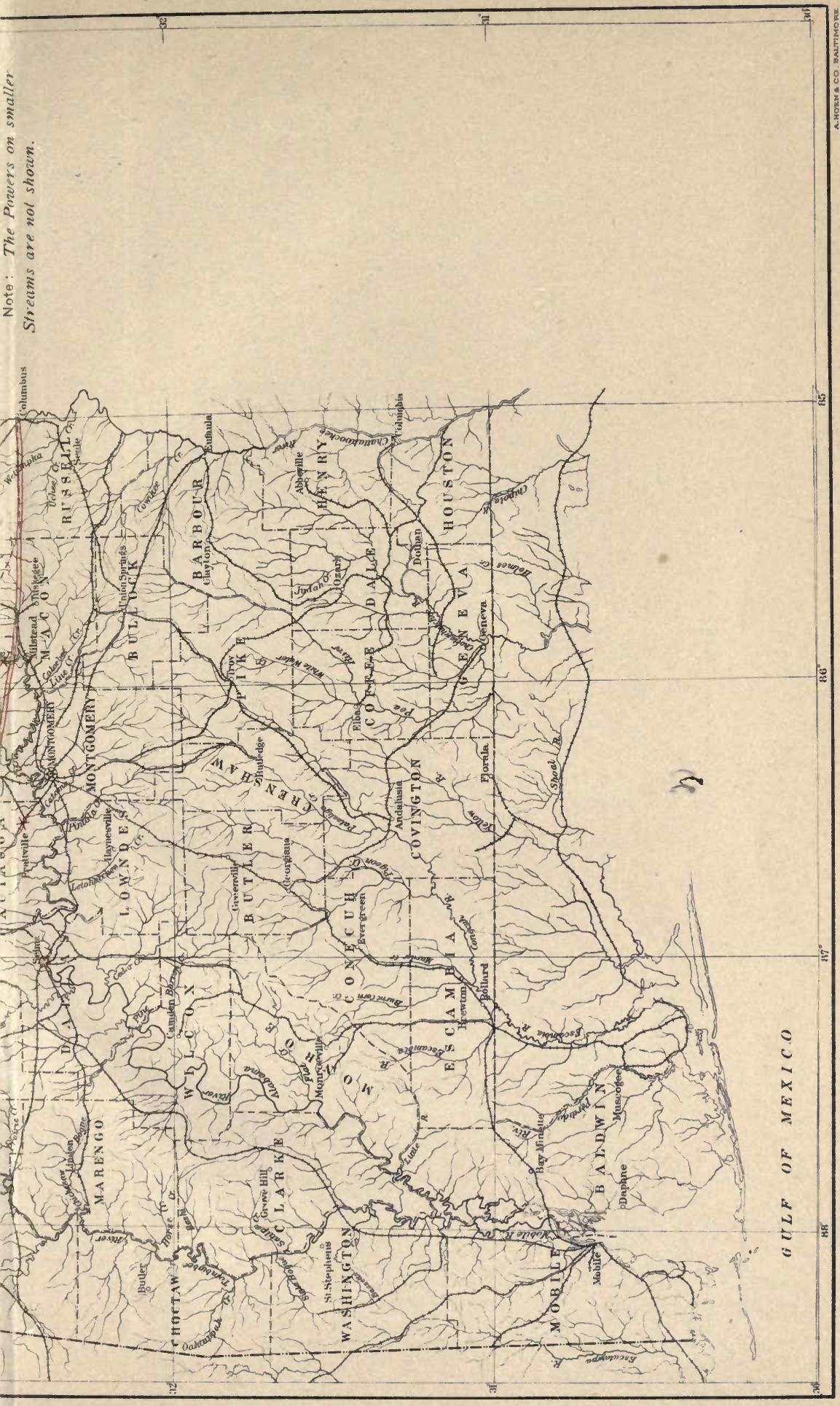


18R2:Y L'NIVERSITY

CALIFORNI 


\section{TABLE OF CONTENTS.}

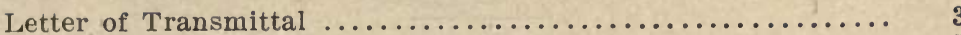

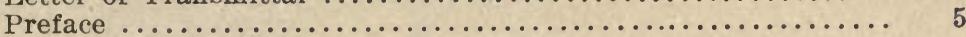

Chapter I.

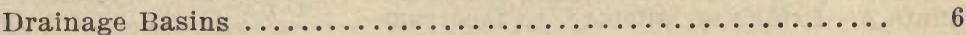

Streams and Water Powers......................... 9

Explanation of Station Records and Tabular Statements de-

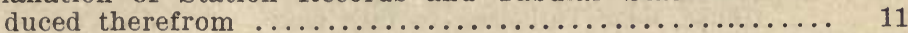

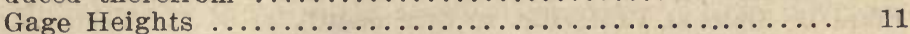

Discharge Measurements ...................... 12

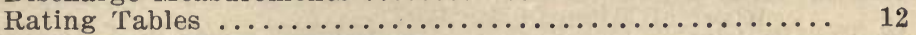

Estimated Monthly Discharge .................... 13

Chapter II.

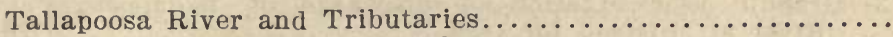

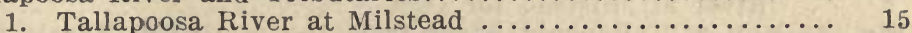

2. Tallapcosa River near Susanna ................ 28

3. Tallapoosa River near Sturdevant.............. 30

4. Survey of the Tallapoosa River in Alabama........ 32

5. Big sandy Creek near Dadeville .................. 39

6. Hillabee Creek near Alexander City ............. 46

7. Alabama Tributaries of the Tallapoosa River from

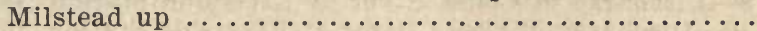

Chapter iII.

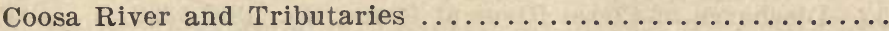

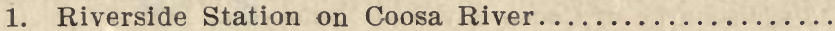

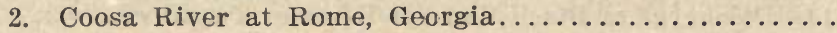

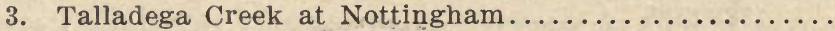

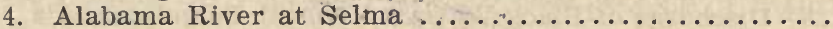

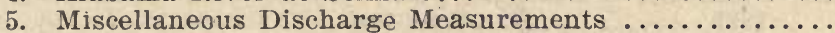

6. Tributaries of the Coosa River from Wetumpka up....

7. Water Powers on Tributaries of Coosa River.........

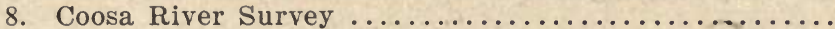

9. Table of Discharge and Net Horse Power at thity-one Locks and Proposed Locks on the Coosa River, at Lowest Water of 1897 and $1900 \ldots \ldots \ldots \ldots \ldots \ldots$.

Chapter IV.

Cahaba River:

1. Cahaba River at Centerville $\ldots \ldots \ldots \ldots \ldots \ldots \ldots \ldots . .69$

2. Survey of the Cahaba River $\ldots \ldots \ldots \ldots \ldots \ldots \ldots \ldots \ldots 1$

Chapter V.

Black Warrior River and Tributaries ................. 104

1. Tuscaloosa Station on Black Warrior River........ 104

2. Black Warrior River at Cordova .............. 120

3. Survey of che Black Warrior River .................. 124

4. Appendix to Black Warrior Report ............. 127

5. Black Warrior River Tributaries............... 128

Tombigbee River:

Chapter Vi.

1. Tombigbee River at Columbus, Miss............ 128

2. Tombigbee River near Epes ..................... 133

3. Tributaries of the Tombigbee $\ldots \ldots \ldots \ldots \ldots \ldots \ldots \ldots 138$

Chapter VII.

Tennessee River and Tributaries:

1. Tennessee River and Tributaries:

2. Shoals in Tennessee River near Florence ........... 161

3. Tributaries of Tennessee River .................... 164

Chapter VIII.

List of Utilized Water Powers, arranged by Counties. 


\section{LIST OF ILLUSTRATIONS.}

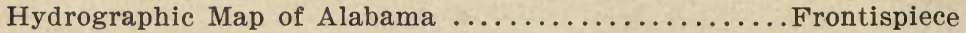

Plate A-Bridge at Milstead, to face page .................... 15

Plate $\mathrm{B}$-Rapids at Tallassee, to face page .................. 32

Plate C-Montgomery Power Company's Dam and Power House, near Tallassee, to face page............ 33

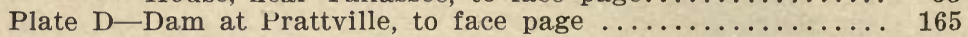

Fig. 1-Graphic representation of discharge of Tallapoosa River at Milstead for $1897 \ldots \ldots \ldots \ldots \ldots \ldots \ldots \ldots$

Fig. 2-Graphic representation of discharge of Tallapoosa

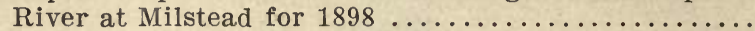

Fig. 3-Graphic representation of discharge of Tallapoosa River at Milstead for $1899 \ldots \ldots \ldots \ldots \ldots \ldots \ldots$.

Fig. 4-Map of Tallapoosa River from top of Griffin Shoals,

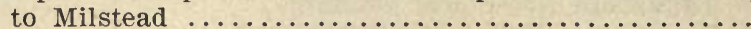

Figs. 5 and 6-Profile of Tallapoosa River from top of Griffin

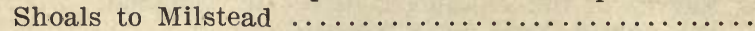

Fig. 8-Profile of Big Sandy Creek, Tallapoosa County.......

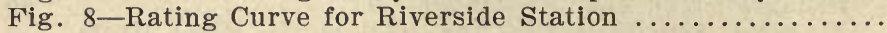

Fig. 9-Discharge of Coosa River at Riverside Station, 1897..

Fig. 10-Discharge of Coosa River at Riverside Station, 1898..

Fig. 11-Discharge of Coosa River at Riverside Station, 1899..

Fig. 12-Discharge of Coosa River at Rome, Ga., 1897-1898...

Fig. 13-Discharge of Coosa River at Rome, Ga., $1899 \ldots \ldots \ldots$.

Fig. 14-Discharge of Black Warrior River at Tuscaloosa, Ala.,

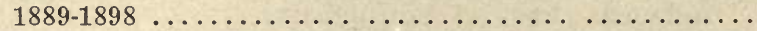

Fig. 15-Discharge of Black Warrior River at Tuscaloosa, Ala.,

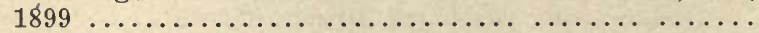

Fig. 16-Discharge Tennessee River at Chattanooga, 1891-1898

Fig. 17-Map showing shoals in Tennessee River near Florence 


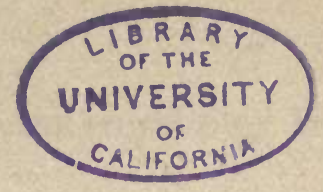

PREFACE.

Very recently two large Water Powers have been developed on the Tallapoosa River, one of which is at Tallassee, Ala., and the other is three miles above Tallassee. These developments have awakened considerable interest in the undeveloped powers of the State, and this Bulletin undertakes to answer in a general way the numerous inquiries concerning them. Some of the largest of these unpublished powers are:

Poiver Site No. 3, on Tallapoosa River, at Double Bridges, about ten miles above-Tallassee, where a head of 40 feet can be obtained. And other similar powers farther up the river.

Black and Sanford Shoal on Big Sandy Creek, near Dadeville, with 80 feet of fall.

Thirty-one locks on the Coosa River, capable of furnishing I,300 to 4,500 horse power each, or an aggnegate of 100,000 horse power during low season of an ordinary year like 1900.

Seven power sites on the Cahaba River capable of furnishing from 500 to I, IOO horse power each.

Squaw Shoals on the Black Warrior, with 43 feet of fall.

Also the following shoals on the Tennessee River:

\begin{tabular}{|c|c|c|c|}
\hline & Fall in & $\begin{array}{c}\text { Minimum } \\
\text { H. P. } \\
\text { dryest }\end{array}$ & $\begin{array}{c}\text { Minimum } \\
\text { H. P. } \\
\text { average }\end{array}$ \\
\hline Shoal: & feet. & years. & years. \\
\hline Elk River Shoal & 26 & 15,600 & 30,550 \\
\hline Big Muscle Shoal ... & 85 & 51,000 & 99,875 \\
\hline Little Muscle Shoal ........... & 23 & 13,800 & 27,025 \\
\hline Colbert Shoal $\ldots \ldots \ldots \ldots \ldots \ldots$ & 21 & 12,600 & 24,675 \\
\hline
\end{tabular}

These and other powers will be described more fully in Chapters II to VIII.

The water powers of Alabama are conveniently located for running cotton factories and other manufacturing plants, and also for generating electricity that can be transmitted to cities for power, light, etc. The larger powers are all close to water transportation, and are also on important railroads. These advantages will naturally make them more valuable than if they were otherwise located.

B. M. HALL,

Nov. I, 1902. 
CHAP'TER I.

DRAINAGE BASINS, STREAMS, AND WATER POWERS.

DFAINAGE BASINS.

The five principal drainage basins of the State are:

First-The Apalachicola Basin, diaining to the Chattahoochee and Apalachicola River, and entering the Gulf at Apalachicola, Fla.

Second-The Choctawhatchee Basin, draining to the Gulf through Choctawhatchee Bay.

Third-The Pensacola Basin, draining to Pensacola Bay and Perdido Bay, near Pensacola, Fla.

Fourth-The Mobile Basin, including the waters of Tallapoosa, Coosa, Cahaba, Alabama, Warrior, and Tombigbee Rivers, and draining into the Gulf at Mobile, Ala.

Fifth-The Tennessee Basin, draining into the Tennessee River, and thence through the Mississippi to the Gulf at New Orleans.

The water powers of the State are mainly in the Mobile and Tennessee Basins, which practically cover the entire State, except a small area in the southeast corner.

The area of crystalline rocks in Alabama is a triangle on the east side of the State, including Cleburne, Randolph, Chambers, Lee, Tallapoosa, Clay, Coosa, and parts of Elmore, Chilton, and Talladega counties. The "fall line," or escarpment dividing the Crystalline region from the Cretaceous formation of the Coastal plain on the southwest, runs from Columbus, Ga., crossing the Tallapoosa River at Tallassee, and the Coosa at Wetumpka. The northwestern boundary of the area of the Crystalline rocks which divides it from the Paleozoic formations, recrosses the Coosa River near Marble Valley postoffice, in Coosa county, and runs in a northeasterly direction towards Cedartown, Ga., crossing the Alabama line near Warner.

The line between the Paleozoic region and the Cretaceous formation runs from a point near Strasburgh, in Chilton county, 
in a northwesterly direction to Tuscaloosa, thence in a northerly direction to a point near Tuscumbia, and thence northwesterly to the Mississippi line.

The southwestern boundary of the Cretaceous passes from Fort Gaines, approximately, through Clayton, Troy, Snow Hill, and Livingston, in a northwesterly direction.

It may be said in a general way that the streams have their greatest falls in passing from an older to a younger geological formation. Tallassee Falls, on the Tallapoosa, and Wetumpka Falls, on the Coosa, are made in passing from the Crystalline to the Cretaceous. Those on Talladega Creek and other small. streams in entering the Coosa Valley from the southeast in Talladega, Calhoun, and Cleburne counties, are from the Crystalline to the Paleozoic. The shoals above Centerville, on the Cahaba, above Tuscaloosa, on the Black Warrior, and near Tuscumbia, on the Tennessee River, are made in passing from the Paleozoic to the Cretaceous. As the Coosa River runs off of the Paleozoic on to the Crystalline near Talladega Springs, the shoals above this point reverse the general order by being made in passing from a younger to an older formation.

\section{STREAMS AND WATER POWERS.}

The following is a statement, according to water--shed, of the important streams and such data concerning them as can be compiled from the work of the Alabama Geological Survey, the United States Weather Bureau, and the United States Engineering Corps, combined with the hydrographic investigations of the United States Geological Survey under the direction of the compiler of this report. Aside from certain surveys made to obtain maps and profiles of Tallapoosa River and Big Sandy Creek, the work done by the Hydrographic Division of the United States Geological Survey in this State deals exclusively with the amount of water flowing in the streams, and is intended to give a safe basis for calculation of low water volumes at all seasons of the year, and for several consecutive years, in order to arrive at their value for water power, irrigation, municipal supply, mining, navigation, etc. In .order to do this certain convenient stations lave been established on important rivers. At each of these stations a gage rod is set to show the fluctuations of the streams; and a gage reader is employed to observe the height of the water every morning at the same hour, 
and to make a weekly report of the same to the Hydrographerin-charge. As far as possible the river stations of the United States Weather Bureau and the United States Engineer Corps have been utilized for this purpose. From time to time the Hydrographer or one of his field assistants, visits the station and makes an accurate meter discharge measurement of the stream, noting the height of the water on the gage at the time the discharge measurement is made. After a number of such discharge measurements have been made at different gageheights, a rating table is made from the data thus obtained, which gives the amount of water ffowing in the stream, at that station, for any gage-height shown on the rod. Thus, by inspection of the table of daily gage-heights, the flow of the stream is shown for every day in the year, or years, covered by the observation of gage-height. At seasons of uniform low water, when the daily fluctuations of the rod are very slight for weeks at a time, discharge measurements are made of the stream at many points above and below the gage station in order to establish a relation between the discharge at these points and at the station. In like manner the principal tributaries are measured for the same purpose, where it is practicable to do so. In this way it is possible to arrive at a close estimate of the flow of all the streams of the water-shed, and make a rating of the gage for each that will represent its flow under average conditions, not including the floods caused by local rains. Such tributaries as have not been measured can be estimated by water-shed comparison with similar tribitaries that have been measured.

In the following statement the actual gage-heights and discharge measurements are given in order to show the data upon which the conclusions are based. The regular gage stations that have been utilized are:

Station.

Stream.

observer.

Paid by.

1-Milstead, Ala..... Tallapoosa River Seth Johnson... Ala. Geo. Sv.

2 -Sturdevant, Ala.... Tallapoosa River B. F. Neighbors. Ala. Geo. Sv.

3-Dadeville, Ala...... Big Sandy Creek T. H. Finch..... Ala. Geo. Sv.

4-Alexander City, Ala.Hillabee Creek.. J. H. Chisolm... Ala. Geo. Sv.

5-Nottingham, Ala... Talladega Creek. R. M. McClatchy.Ala. Geo. Sv.

6-Riverside, Ala...... Coosa River......J. W. Foster.....Ala. Geo. Sv.

7 -Cordova, Ala....... Black Warrior R A. B. Logan.....Ala. Geo. Sv.

8-Montgomery, Ala... Alabama River.. U. S. W. B ...... U. S. W. B.

9-Selma, Ala......... Alabama River.. U. S. W. B...... U. S. W. B.

10-Tuscaloosa, Ala.... Black Warrior R W. S. wyman, JrU. S. Eng. C.

11-Epes, Ala ......... Tombigbee River J. C. Horton......A. G. S. Ry.

12-Rome, Ga......... Coosa River..... W. M. Towers... U. S. W. B.

13-Chattanooga, Tenn. Tennessee River. U. S. W. B..... U. S. W. B. 
As the investigations in this State have been confined so far mainly to the Mobile and Tennessee basins, only the streams of these basins will be considered in the following discussion. It is to be remembered that from West Point, Ga., southwards, the line of Alabama is on the west bank of the Chattahoochee River, along the line where ordinary vegetation ceases to grow. This leaves all of the water power of the main stream on Georgia territory. There are many creeks flowing into the river from Alabama, some of which have considerable fall, as they come from a high plateau. Holland Creek, opposite Columbus, Ga., furnishes the Columbus water supply by gravity, having a fall of II 7 feet in less than four miles. No doubt many of the others have as much fall, but as they have not been examined, a report on them cannot be made at present, but a recent reconnoissance along the Chattahoochee gives the following estimate of power obtained from some of them, I2 hours per day for each foot of fall, if the water is stored during the 12 idle hours:

Big Uchee Creek, Russell County...... 7 H. P. per foot of fall. Ihagee Creek, Russell County........ $2 \mathrm{H}$. P. per foot of fall. Hatchechubbee Creek, Russell County... 7 H. P. per foot of fall. Cowikee Creek, Barbour County........ 11 H. P. per foot of fall. Yattayabba Creek, Henry County...... 9 H. P. per foot of fall. Omussee Creek, Henry county........ $7 \mathrm{H}$. P. per foot of fall.

EXPLANATION OF STATION RECORDS AND TABULAR STATEMENTS DEDUCED THEREFROM.

GAGE HEIGHTS.

The "Table of Gage Heights" is a record of the height of water on a gage rod, graded to feet and hundredths of a foot, set into the river vertically, and fastened permanently to a convenient tree or pier. The rod is read every day in the year, at the same time of day, which is about 8 o'clock in the morning. Inches are not used in these records, as the daily height of water on the gage is written in feet and decimals of a foot. 


\section{DISCHARGE MEASUREMENTS.}

These records show the date, the gage height at time of measurement, and the amount of water in cubic feet per second, or "second-feet," that is found by the measurement to be flowing in the river. (Second-feet means the same as cubic feet per second.) If we imagine a small stream filling a rectangular flue I foot wide and I foot deep, we have a stream whose sectional area is I square foot. The volume of this stream will vary in proportion to the speed with which the water flows through the flume. If the water is moving at a velocity of I foot per second, the flowl or volume of water is I cubic foot per second, and would fill a vessel 5 feet wide, 5 feet long, and 4 feet deep in just Ioo seconds, as such a vessel would hold Ioo cubic feet of water. If the water in the flume I foot wide and I foot deep flows with a velocity of 2 feet per second, the volume will be 2 cubic feet per second, or 2 second-feet, and so on for any other velocity. In the same way if the flume is 20 feet wide, and 5 feet deep, its sectional area will be Ioo square feet, and if the average velocity is 3 feet per second, the volume will be 300 cubic feet per second, or 300 second-feet. In each of the discharge measurements here enumerated, a cross-section of the stream is measured, and velocities taken with an electric current-meter at many points of the cross-section. Instead of multiplying the entire cross-section by an average velocity, the area was divided up into a large number of small sections by soundings from 5 to ro feet apart, and the area of each of the small sections multiplied by the velocity at the small section, thus giving the second-feet flowing in each small section. The sum of the discharges of all the small sections makes the total discharge of the stream.

\section{RATING TABLE.}

This is a table showing the discharges in second-feet (cubic feet per second) for all stages of water on the gage. Hence when the gage heights are known, the corresponding discharges can be taken from the rating table and written opposite each daily gage height, thus giving the flow in cubic feet per second on each day in the entire year. 
FSTIMATED MONTHLY DISCHARGE, ETC.

This table gives in the first three columns, the maximum, minimum, and mean discharge for each month in cubic feet per second (second feet.) Column No. 4 gives the "total acre feet" flowing down the stream during each month. An "acre-foot" is the amount of water that would be necessary to cover one acre with a depth of one foot, which is 43,560 cubic feet. It furnishes a convenient unit for storage, where the water is to be used for irrigation. A cubic foot is practically 7.48 gallons, and is usually estimated at 7.5 gallons. An acre-foot is 43,560 cubic feet, or 320,851 gallons. One cubic foot per second flowing for 24 hours will cover an acre to a depth of 1.98 feet. It is therefore customary in round numbers to state that a cubic foot per second for a day of 24 hours is equivalent to 2 acre feet. Now, as one inch of rainfall per hour falling for $\mathrm{I} 2$ hours would cover one acre a foot deep, it is evident that rainfall at the rate of $I$ inch per hour will produce a flow of I cubic foot per second, or 2 acre feet per 24 hours for each acre of watershed, no allowance being made for evaporation or percolation. It is also convenient to remember that $\mathrm{I}, 000,000$ gallons in a reservoir are equal to a little more than 3 acre feet (3.069). In a general way it may be said that water stored in reservoirs is reckoned in acre-feet for irrigation, cubic feet for water power, and in millions of gallons for city watersupply.

Columns 5 and 6 give the "run-off" from the drainage area. The run-off in inches and decimals of an inch is given, just as rainfall is given. For instance, a run-off of 2.23 inches from a given drainage area, means that enough water ran off during the month to have covered the entire drainage area or water-shed to a depth of 2.23 inches. This is convenient in estimating the proportion of the rain-fall on any drainage area that can be stored for irrigation, city water supply, or other purposes. The run-off in second-feet per square mile of drainage area, is obtained by dividing the mean discharge for the month by the number of square miles in the drainage area, and is useful in estimating the mean discharge of a tributary whose drainage area is known, and in comparing different drainage areas. The "run-off" is not a fixed percentage of the rainfall, but is that part of the rainfall which is not lost by evaporation into the air, or by percolation in subterranean outlets. Being a remainder and 
not a percentage, it necessarily forms a much larger proportion of a heavy annual rainfall than it does of a small annual rainfall. For instance, in the Crystalline region of Georgia or Alabama where the annual precipitation is 45 to 55 inches, the run-off from the water-sheds is equal to fully one-half of the rainfall, while in regions having a precipitation of only io to 20 inches annually, the run-off is frequently less than one-fifth of the rainfall. Again, the geological character of the water-shed makes a vast difference in the run-off, even where the annual rainfall is the same, and where practically the same conditions of climate, topography, forest area and cultivation exist. There will be a smaller run-off from the water-shed having permeable geological strata underneath it, into which a part of the rain water can percolate, and furnish the supply to artesian wells in the lower country under which the same strata run, without regard to surface topography. In a comparison of two such water-sheds, one in the crystalline region, and the other in a regularly stratified formation, the difference of run-off should form a basis for estimating the artesian supply obtainable from the latter as a fountain head. 



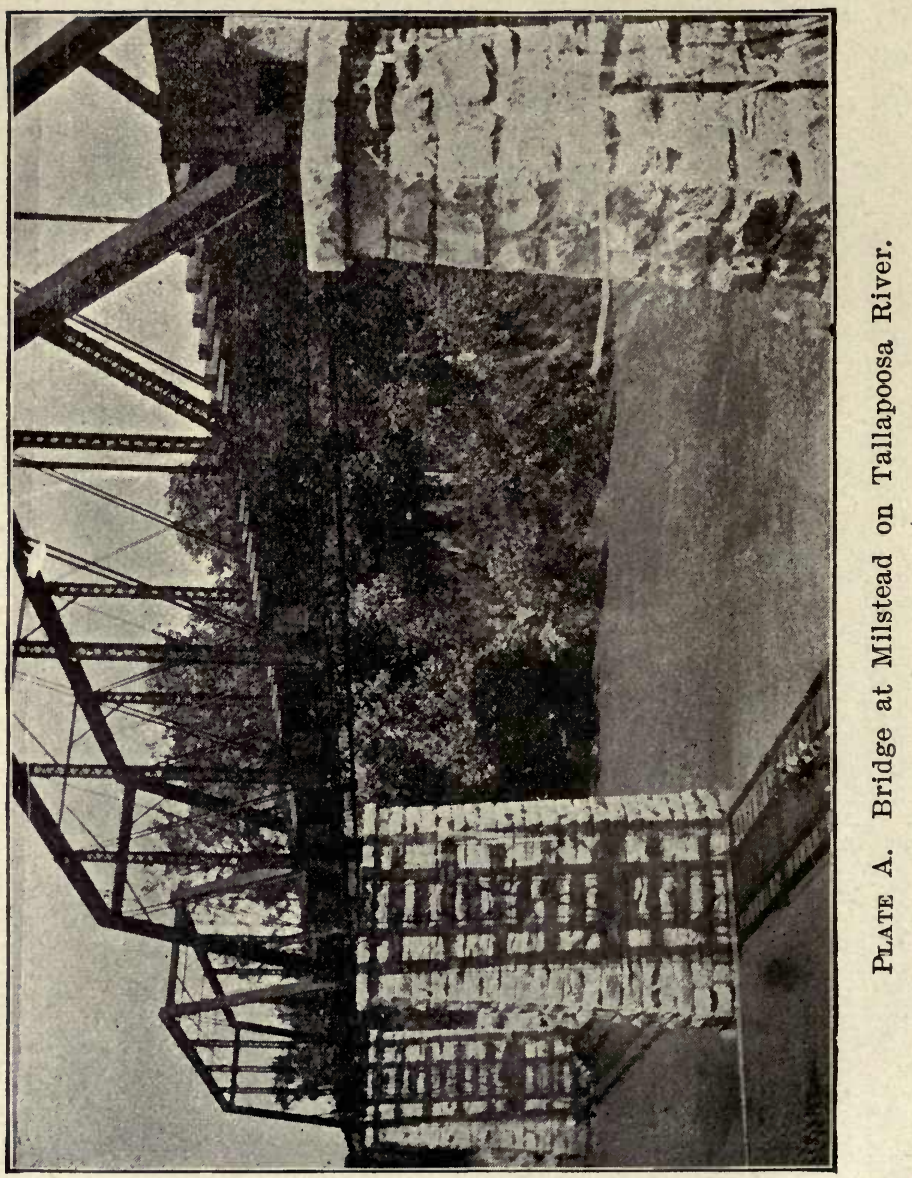


CHAPTER II.

TALLAPOOSA RIVER AND TRIBUTARIES.

1. TALI APOOSA RIVER AT MILSTEAD, ALABAMA.

Tallapoosa River rises in the west-central part of Georgia and flows in a southwesterly direction into Alabama, where it joins the Coosa, to form Alabama River, 6 miles above Montgomery, Alabama. Its upper tributaries drain an area between the Chattahoochee and Coosa basins. At Tallassee, Alabama, it crosses the southern fall line. The shoals at this place have a fall of 60 feet, forming an obstruction to navigation. The drainage area is largely wooded, with cultivated fields at short intervals. A gaging station was established at Milstead on August 7, 1897, at the bridge of the Tallassee \& Montgomery Railway, about one-fourth of a mile from Milstead, Alabama. The bridge is of iron, two spans of about I55 feet each, with short wooden trestles at each end. The initial point of measurement is the end of the iron bridge, left bank, downstream side. The rod of wire gage is fastened to outside of guard rail on downstream side of bridge. The bench mark is top of second cros beam from left-bank pier, downstream end, and is 60.00 feet above datum. The channel is straight at the bridge, and bends above and below. The current is sluggish at low water and obstructed by center pier of bridge. The banks are high, but overflow at extreme high water for several hundred feet on each side. The bed is fairly constant, and all water is confined to the main channel by railroad embankments. The observer is Seth Johnson, a farmer and fruit grower, Milstead, Alabama. The plate A opposite shows this station.

The following discharge measurements were made during 1897 by Max Hall :

May 3, gage height, 6.20 feet; discharge, 7,333 second-feet.

July 15, gage height, 1.95 feet; discharge, 1,692 second-feet.

August 7, gage height, 2.42 feet; discharge, 2,292 second-feet.

September 4, gage height, 1.60 feet; discharge, 1,271 second-feet.

November 23, gage height, 1.20 feet; discharge, 677 second-feet.

December 16, gage height, 3.58 feet; discharge, 4,210 second-feet. 
*Daily gage height, in feet, of Tallapoosa River at Milstead, Alabama, from August to December, 1897.

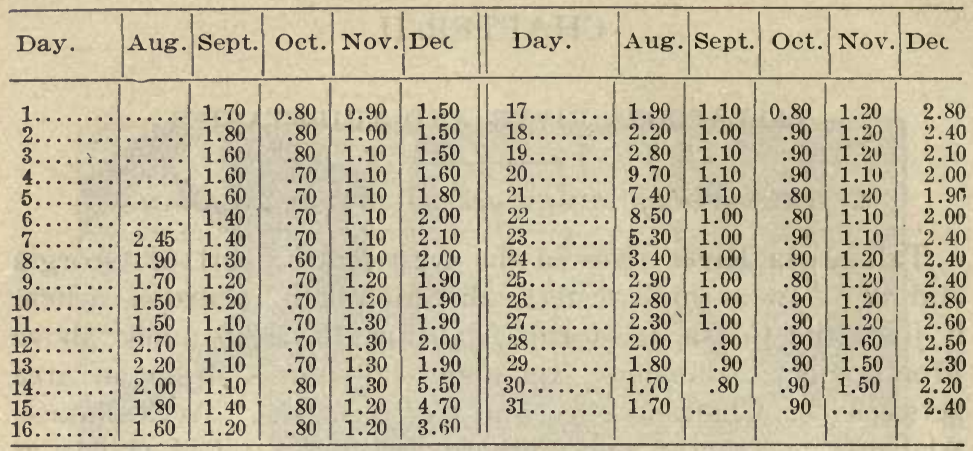

*See explanation, pages 11 to 14 .

Rating table for Tallapoosa River at Milstead, Alabama, for $189 \%$.

\begin{tabular}{|c|c|c|c|c|c|c|c|}
\hline 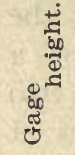 & 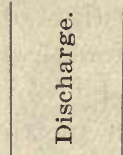 & 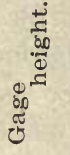 & 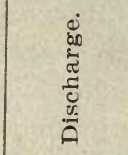 & & 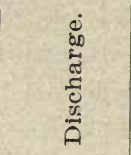 & 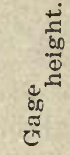 & 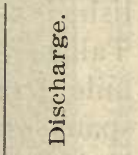 \\
\hline Feet. & Second-feet & Feet. & Second-feet & Fee! & second-feet. & Feet. & Second-feet. \\
\hline 0.5 & 330 & 1.5 & 1,070 & 3.0 & 3,129 & 5.0 & 5,90 \\
\hline 0.6 & 350 & 1.6 & 1,200 & 3.2 & 3,407 & 5.2 & 6,1 \\
\hline 0.7 & 380 & 1.7 & 1,333 & 3.4 & 3,685 & 5.4 & 6,4 \\
\hline 0.8 & 420 & 1.8 & 1,467 & 3.6 & 3,96 & 5.6 & 6,7 \\
\hline 0.9 & 470 & 1.9 & 1,600 & 3.8 & & 5.8 & 7,0 \\
\hline 1.0 & 530 & 2.0 & 1,733 & 4.0 & 4,519 & 6.0 & $7,2 !$ \\
\hline 1.1 & 620 & 2.2 & 2,007 & 4.2 & 4,79 & 7.0 & 8,6 \\
\hline 1.2 & 720 & 2.4 & 2,285 & 4.4 & 5,075 & 8.0 & 10,079 \\
\hline 1.3 & 830 & 2.6 & 2,573 & 4.6 & 5,353 & 9.0 & 11,469 \\
\hline 1.4 & 950 & 2.8 & 2,851 & 4.8 & 5,631 & & \\
\hline
\end{tabular}

Note-This table applied to the foregoing "Daily gage heights" gives the cubic. feet per second flowing in the river on each date for which the gage height is given. 
The following discharge measurements were made during I 898 by Max Hall and others :

Jan. 19-Gage height, 2.13 feet; discharge, 1,889 second-feet. Feb. 19-Gage height, 2.20 feet; discharge, 2,045 secod-feet. March 18-Gage height, 2.56 feet; discharge, 2,646 second-feet. April 26-Gage height, 5.83 feet; discharge, 6,648 second-feet. May 17-Gage height, 1.55 feet; discharge, 1,059 second-feet. June 22-Gage height, 3.05 feet; discharge, 3,421 second-feet. July 7-Gage height, 1.62 feet; discharge, 1,262 second-feet.

Aug. 5-Gage height, 13.67 feet; discharge, 15,295 second-feet.

Sept. 3-Gage height, 2.76 feet; discharge, 3,010 second-feet.

Nov. 29-Gage height, 5.16 feet; discharge, 5,477 second-feet.

Daily gage height, in feet, of Tallapoosa River at Milstead, Alabama, for 1898.

\begin{tabular}{r|r|r|r|r|r|r|r|r|r|r|r|r|}
\hline Day & Jan. & Feb. & Mar. & April & May & Jun. & July & Aug. & Sept. & Oct. & Nov. & Dec \\
\hline & & & & & & & & \\
\end{tabular}


Rating table for Tällapoosa River at Milstead, Alabama, for 1898.

\begin{tabular}{|c|c|c|c|c|c|}
\hline & 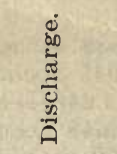 & 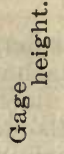 & 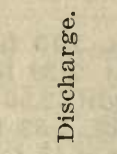 & 造 & $\begin{array}{l}\dot{0} \\
\infty \\
\dot{\sigma} \\
\tilde{0} \\
\overline{0} \\
\tilde{0} \\
\vec{A}\end{array}$ \\
\hline Feel. & Second $F t$. & Feet. & Second $F t$. & Feet. & Second $F t$. \\
\hline 0.8 & 540 & 2.4 & 2,380 & 8.0 & 8,820 \\
\hline 0.9 & 655 & 2.5 & 2,495 & 8.5 & 9,395 \\
\hline 1.0 & 770 & 2.6 & 2,610 & 9.0 & 9,970 \\
\hline 1.1 & 885 & 2.7 & 2,725 & 9.5 & 10,545 \\
\hline 1.2 & 1,000 & 2.8 & 2,840 & 10.0 & 11,120 \\
\hline 1.3 & 1,115 & 2.9 & 2,955 & 10.5 & 11,695 \\
\hline 1.4 & 1,230 & 3.0 & 3,070 & 11.0 & 12,270 \\
\hline 1.5 & 1,345 & 3.5 & 3,645 & 11.5 & 12,845 \\
\hline 1.6 & 1,460 & 4.0 & 4,220 & 12.0 & 13,420 \\
\hline 1.7 & 1,575 & 4.5 & 4,795 & 12.5 & 13,995 \\
\hline 1.8 & 1,690 & 5.0 & 5,370 & 13.0 & 14,570 \\
\hline 1.9 & 1,805 & 5.5 & 5,945 & 13.5 & 15,145 \\
\hline 2.0 & 1,920 & 6.0 & 6,520 & 14.0 & 15,720 \\
\hline 2.1 & 2,035 & 6.5 & 7,095 & 14.5 & 16,295 \\
\hline 2.2 & 2,150 & 7.0 & 7,670 & 15.0 & 16,870 \\
\hline 2.3 & 2,265 & 7.5 & 8,245 & & \\
\hline
\end{tabular}

Note.-This table applied to the foregoing "Daily gage heights" gives the cubic feet per second flowing in the river on each date for which the gage height is given.

The following discharge measurements were made during I899 by Max Hall :

April 17-Gage height, 6.34 feet; discharge, 7,444 second-feet. April 18-Gage height, 5.63 feet; discharge, 6,853 second-feet. May 17-Gage height, 2.80 feet; discharge, 3,000 second-feet.

June 26-Gage height, 2.05 feet; discharge, 1,847 second-feet.

September 9-Gage height, 1.36 feet; discharge, 1,016 second-feet.

November 8-Gage height, 1.25 feet; discharge, 972 second-feet.

December 18-Gage height, 2.66 feet; discharge, 2,844 second-feet. 
Daily gage height, in feet, of Tallapoosa River, at Milstead, Alabama, for 1899.

\begin{tabular}{|c|c|c|c|c|c|c|c|c|c|c|c|c|}
\hline Day & Jan. & Feb. & Mar. & April & May & Jun. & July & Aug. & Sept. & Oct. & Nov. & Dec \\
\hline 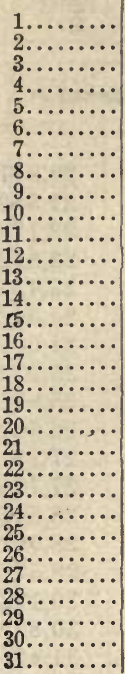 & $\begin{array}{r}5.00 \\
4.60 \\
3.90 \\
3.90 \\
3.90 \\
3.80 \\
7.40 \\
8.00 \\
7.10 \\
6.40 \\
18.50 \\
16.80 \\
13.00 \\
11.60 \\
9.40 \\
7.80 \\
12.70 \\
10.00 \\
8.00 \\
6.50 \\
5.70 \\
5.30 \\
5.10 \\
5.20 \\
5.20 \\
5.10 \\
4.80 \\
4.70 \\
5.10 \\
5.20 \\
6.50\end{array}$ & $\begin{array}{r}17.00 \\
11.60 \\
24.50 \\
20.00 \\
12.90 \\
11.90 \\
17.50 \\
27.00 \\
19.00 \\
13.80 \\
10.00 \\
8.30 \\
7.40 \\
7.00 \\
6.10 \\
10.40 \\
11.50 \\
10.60 \\
9.30 \\
8.30 \\
8.30 \\
8.40 \\
7.60 \\
6.90 \\
6.40 \\
6.30 \\
25.00 \\
37.00 \\
\ldots \ldots . . \\
\ldots \ldots . . \\
\ldots . . .\end{array}$ & $\begin{array}{r}27.00 \\
19.00 \\
13.50 \\
9.60 \\
14.20 \\
13.20 \\
10.10 \\
8.60 \\
7.70 \\
7.20 \\
6.80 \\
6.60 \\
6.50 \\
6.40 \\
7.20 \\
12.20 \\
11.00 \\
10.20 \\
14.80 \\
13.90 \\
10.40 \\
8.30 \\
8.10 \\
12.70 \\
8.70 \\
7.30 \\
6.90 \\
6.80 \\
9.00 \\
8.90 \\
13.85\end{array}$ & $\begin{array}{r}18.00 \\
12.20 \\
6.50 \\
6.40 \\
8.50 \\
9.70 \\
10.30 \\
13.00 \\
13.00 \\
11.20 \\
8.40 \\
7.00 \\
6.50 \\
6.20 \\
6.00 \\
7.10 \\
6.60 \\
5.60 \\
5.50 \\
5.40 \\
5.20 \\
5.00 \\
4.90 \\
6.00 \\
10.00 \\
7.50 \\
6.60 \\
5.80 \\
4.90 \\
4.60 \\
\ldots . .\end{array}$ & $\begin{array}{l}4.30 \\
4.00 \\
3.90 \\
3.80 \\
3.70 \\
3.60 \\
3.50 \\
3.60 \\
3.50 \\
3.40 \\
3.30 \\
3.20 \\
3.10 \\
3.10 \\
3.00 \\
2.90 \\
2.80 \\
2.70 \\
2.60 \\
2.50 \\
2.60 \\
2.70 \\
2.60 \\
3.30 \\
4.60 \\
3.30 \\
2.80 \\
2.60 \\
2.50 \\
2.60 \\
2.50\end{array}$ & $\begin{array}{l}2.40 \\
2.90 \\
2.40 \\
2.40 \\
2.30 \\
2.20 \\
2.10 \\
2.00 \\
1.90 \\
1.70 \\
1.90 \\
2.00 \\
2.00 \\
2.70 \\
2.70 \\
2.60 \\
2.10 \\
1.90 \\
1.80 \\
1.80 \\
1.70 \\
1.50 \\
1.50 \\
1.50 \\
1.50 \\
2.00 \\
2.50 \\
2.50 \\
2.20 \\
2.70 \\
\ldots . .\end{array}$ & $\begin{array}{r}2.80 \\
2.30 \\
1.90 \\
1.80 \\
1.10 \\
1.50 \\
1.50 \\
2.00 \\
2.20 \\
2.00 \\
1.80 \\
1.70 \\
1.50 \\
1.40 \\
1.30 \\
1.30 \\
1.20 \\
1.10 \\
1.60 \\
1.40 \\
8.40 \\
16.75 \\
14.00 \\
16.95 \\
7.90 \\
6.70 \\
6.80 \\
8.40 \\
10.10 \\
5.40 \\
4.40\end{array}$ & $\begin{array}{l}3.00 \\
2.70 \\
3.10 \\
3.10 \\
2.50 \\
2.30 \\
2.20 \\
2.00 \\
1.80 \\
1.60 \\
1.50 \\
1.70 \\
1.60 \\
1.60 \\
1.50 \\
4.00 \\
3.90 \\
2.20 \\
1.90 \\
1.70 \\
1.50 \\
1.60 \\
2.00 \\
2.60 \\
1.90 \\
1.80 \\
3.70 \\
2.80 \\
2.10 \\
1.90 \\
2.30\end{array}$ & $\begin{array}{r}2.40 \\
2.30 \\
2.10 \\
2.00 \\
1.80 \\
1.60 \\
1.60 \\
1.40 \\
1.40 \\
1.30 \\
1.20 \\
1.20 \\
1.20 \\
1.10 \\
1.00 \\
1.00 \\
1.00 \\
1.00 \\
1.10 \\
1.00 \\
.90 \\
1.00 \\
1.00 \\
.90 \\
.90 \\
.90 \\
.90 \\
.80 \\
.80 \\
.80 \\
\ldots . . .\end{array}$ & $\begin{array}{r}0.70 \\
.70 \\
.80 \\
.90 \\
1.00 \\
1.40 \\
1.40 \\
1.30 \\
1.30 \\
1.30 \\
1.40 \\
1.40 \\
1.30 \\
1.30 \\
1.20 \\
1.10 \\
1.20 \\
1.10 \\
1.20 \\
1.30 \\
1.40 \\
1.50 \\
1.60 \\
1.80 \\
1.50 \\
1.40 \\
1.30 \\
1.20 \\
1.50 \\
1.50 \\
1.60\end{array}$ & $\begin{array}{l}1.50 \\
1.40 \\
1.40 \\
1.30 \\
1.30 \\
1.20 \\
1.20 \\
1.20 \\
1.20 \\
1.20 \\
1.30 \\
1.30 \\
1.30 \\
1.30 \\
1.30 \\
1.30 \\
1.60 \\
1.50 \\
1.50 \\
1.50 \\
1.40 \\
1.40 \\
1.60 \\
1.80 \\
2.20 \\
4.60 \\
6.20 \\
4.80 \\
3.60 \\
2.80 \\
\ldots . .\end{array}$ & $\begin{array}{r}2.40 \\
2.50 \\
2.60 \\
2.60 \\
2.90 \\
2.20 \\
2.00 \\
1.90 \\
1.80 \\
1.90 \\
2.00 \\
15.20 \\
13.20 \\
8.20 \\
5.00 \\
3.70 \\
3.00 \\
2.60 \\
2.50 \\
2.70 \\
2.70 \\
2.70 \\
2.60 \\
9.30 \\
9.40 \\
7.20 \\
5.00 \\
4.00 \\
3.50 \\
3.10 \\
2.90\end{array}$ \\
\hline
\end{tabular}

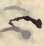


Rating table for Tallapoosa River at Milstead, Alabama, for 1899.

\begin{tabular}{|c|c|c|c|c|c|}
\hline & 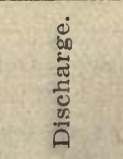 & 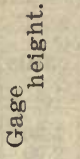 & 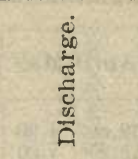 & 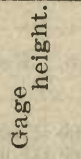 & 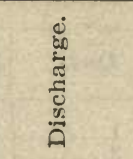 \\
\hline Feet. & Second $F$. & Feet. & Second Feet. & Feet. & Second $F t$. \\
\hline 0.7 & 320 & 6.5 & 7,437 & 17.5 & 20,977 \\
\hline 0.8 & 430 & 7.0 & 8,052 & 18.0 & 21,582 \\
\hline 0.9 & 550 & 7.5 & 8,667 & 18.5 & 22,179 \\
\hline 1.0 & 672 & 8.0 & 9,282 & 19.0 & 22,812 \\
\hline 1.1 & 795 & 8.5 & 9,897 & 19.5 & 23,427 \\
\hline 1.2 & 918 & 9.0 & 10,512 & 20.0 & 24,042 \\
\hline 1.3 & 1,041 & 9.5 & 11,127 & 20.5 & 24,657 \\
\hline 1.4 & 1,164 & 10.0 & 11,742 & 21.0 & 25,272 \\
\hline 1.5 & 1,287 & 10.5 & 12,357 & 21.5 & 25,887 \\
\hline 1.6 & 1,410 & 11.0 & 12,972 & 22.0 & 26,502 \\
\hline 1.7 & 1,533 & 11.5 & 13,587 & 22.5 & $27 ; 117$ \\
\hline 1.8 & 1,656 & 12.0 & 14,202 & 23.0 & 27,732 \\
\hline 1.9 & 1,779 & 12.5 & 14,817 & 23.5 & 28,347 \\
\hline 2.0 & 1,902 & 13.0 & 15,432 & 24.0 & 28,962 \\
\hline 2.5 & 2,517 & 13.5 & 16,047 & 24.5 & 29,577 \\
\hline 3.0 & 3,132 & 14.0 & 16,662 & 25.0 & 30,192 \\
\hline 3.5 & 3,747 & 14.5 & 17,277 & 25.5 & 30,807 \\
\hline 4.0 & 4,362 & 15.0 & 17,892 & 26.0 & 31,422 \\
\hline 4.5 & 4,977 & 15.5 & 18,507 & 26.5 & 32,037 \\
\hline 5.0 & 5,592 & 16.0 & 19,122 & 27.0 & 32,652 \\
\hline 5.5 & 6,207 & 16.5 & 19,737 & 27.5 & 33,287 \\
\hline 6.0 & 6,822 & 17.0 & 20,352 & 27.9 & 33,779 \\
\hline
\end{tabular}

Note-This table applied to the foregoing "Daily gage heights" gives the cubic feet per second flowing in the river on each date for which the gage height is given.

During the year Igoo the following discharge measurements were made by Max Hall:

Feb. 23-Gage heigst, 9.20 feet; discharge, 9,956 second-feet.

March 5-Gage height, 6.70 feet; discharge, 7,088 second-feet.

Dec. 3-Gage height, 2.95 feet; discharge, 3,031 second-feet. 
Daily gage height, in feet, of Tallapoosa River near Milstead, Ala bama, for 1900.

\begin{tabular}{r|r|r|r|r|r|r|r|r|r|r|r|r}
\hline \multicolumn{1}{r|}{ Day. } & Jan. & Feb. & Mar. & April & May & June & July & Aug. & Sept. & Oct. & Nov. & Dec \\
& &
\end{tabular}

The following measurements were made by Max Hall and James R. Hall during rgor:

Feb. 12-Gage height, 10.70 feet; discharge, 11,759 second-feet.

March 13-Gage height, 5.55 feet; discharge, 5,644 second-feet.

October 29-Gage height, 1.70 feet; discharge, 1,583 second-feet. 
Daily gage height of Tallapoosa River at Milstead, Ala., for 1901.

\begin{tabular}{r|r|r|r|r|r|r|r|r|r|r|r|r}
\hline Day. & Jan. & Feb. & Mar. & Apr & May. & Jun . July & Aug. & Sept. & Oct. & Nov. & Dec. \\
&
\end{tabular}


Rating table for Tallapoosa River at Milstead, Ala., for 1900 and 1901.

\begin{tabular}{|c|c|c|c|c|c|}
\hline 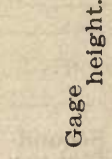 & 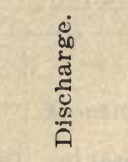 & 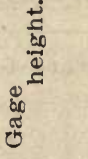 & 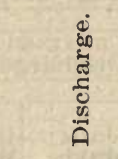 & 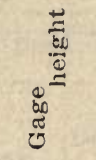 & 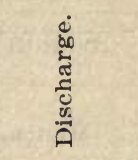 \\
\hline Feet. & Second $\boldsymbol{F}^{\prime} t$. & Feel. & Second $F t$ & Feet. & Second Ft. \\
\hline 1.5 & 1,337 & 4.1 & 3,262 & 18.0 & 19,900 \\
\hline 1.6 & 1,450 & 4.2 & 4,375 & 19.0 & 21,025 \\
\hline 1.7 & 1,562 & 4.3 & 4,487 & 20.0 & 22,150 \\
\hline 1.8 & 1,675 & 4.4 & 4,600 & 21.0 & 23,275 \\
\hline 1.9 & 1,787 & 4.5 & 4,712 & 22.0 & 24,400 \\
\hline 2.0 & 1,900 & 4.6 & 4,825 & 23.0 & 25,525 \\
\hline 2.1 & 2,012 & 4.7 & 4,937 & 24.0 & 26,650 \\
\hline 2.2 & 2,125 & 4.8 & 5,050 & 25.0 & 27,775 \\
\hline 2.3 & 2,237 & 4.9 & 5,162 & 26.0 & 28,900 \\
\hline 2.4 & 2,350 & 5.0 & 5,275 & 27.0 & 30,025 \\
\hline 2.5 & 2,462 & 5.5 & 5,837 & 28.0 & 31,150 \\
\hline 2.6 & 2,575 & 6.0 & 6,400 & 29.0 & 32,275 \\
\hline 2.7 & 2,687 & 6.5 & 6,962 & 30.0 & 33,400 \\
\hline 2.8 & 2,800 & 7.0 & 7,525 & 31.0 & 34,525 \\
\hline 2.9 & 2,912 & 7.5 & 8,087 & 32.0 & 35,650 \\
\hline 3.0 & 3,025 & 8.0 & 8,650 & 33.0 & 36,775 \\
\hline 3.1 & 3,137 & 8.5 & 9,212 & 34.0 & 37,900 \\
\hline 3.2 & 3,250 & 9.0 & 9,775 & 35.0 & 39,025 \\
\hline 3.3 & 3,362 & 10.0 & 10,900 & 36.0 & 41,150 \\
\hline 3.4 & 3,475 & 11.0 & 12,025 & 37.0 & 41,275 \\
\hline 3.5 & 3,587 & 12.0 & 13,150 & 38.0 & 42,400 \\
\hline 3.6 & 3,700 & 13.0 & 14,275 & 39.0 & 43,525 \\
\hline 3.7 & 3,812 & 14.0 & 15,400 & 40.0 & 44,650 \\
\hline 3.8 & 3,925 & 15.0 & 16,525 & 41.0 & 45,775 \\
\hline 3.9 & 4,037 & 16.0 & 17,650 & 42.0 & 46,900 \\
\hline 4.0 & 4,150 & 17.0 & 18,775 & 43.0 & 48,025 \\
\hline
\end{tabular}

Note-This table applied to the foregoing "Daily gage heights" gives the cubic feet per second flowing in the river on each date for which the gage height is given. 
*Estimated monthly discharge of Tallapoosa River at Milstead, Ala.

[Drainage area, 3,840 square miles.]

\begin{tabular}{|c|c|c|c|c|c|c|}
\hline \multirow[b]{2}{*}{ Month. } & \multicolumn{3}{|c|}{ Discharge in second-feet. } & \multirow[b]{2}{*}{$\begin{array}{l}\text { Total in } \\
\text { acre- } \\
\text { feet. }\end{array}$} & \multicolumn{2}{|c|}{ Run-off. } \\
\hline & $\begin{array}{l}\text { Maxi- } \\
\text { mum. }\end{array}$ & $\begin{array}{l}\text { Mini- } \\
\text { mum. }\end{array}$ & Mean. & & $\begin{array}{l}\text { Depth } \\
\text { in } \\
\text { inches. }\end{array}$ & $\begin{array}{l}\text { Second. } \\
\text { feet per } \\
\text { square } \\
\text { mile. }\end{array}$ \\
\hline 1897. & & & & & & \\
\hline August, $7-31 \ldots \ldots \ldots$ & 12,440 & 1,070 & 3,173 & 157,340 & 0.77 & 0.83 \\
\hline September ......... & 1,467 & 420 & 742 & 44,155 & 0.21 & 0.19 \\
\hline October ............ & 470 & 380 & 424 & 26,070 & 0.12 & 0.11 \\
\hline November $\ldots . . . .$. & 1,200 & 470 & 729 & 43,379 & 0.21 & 0.19 \\
\hline $\begin{array}{l}\text { December } \cdots \cdots \\
1898 \text {. }\end{array}$ & 6,604 & 1,070 & 2,214 & 136,135 & 0.67 & 0.58 \\
\hline January........ & 4,105 & 1,575 & 2,426 & 149,170 & 0.72 & 0.63 \\
\hline February....... & 2,265 & 1,690 & 1,912 & 106,187 & 0.52 & 0.50 \\
\hline March $\ldots \ldots \ldots \ldots$ & 5,715 & 1,575 & 2,313 & 142,222 & 0.69 & 0.60 \\
\hline April $\ldots \ldots \ldots \ldots \ldots$ & 21,240 & 2,150 & 5,748 & 342,029 & 1.67 & 1.50 \\
\hline May ............. & 2,610 & 885 & 1,493 & 91,802 & 0.45 & 0.39 \\
\hline June $\ldots \ldots \ldots$. . . & 3,070 & 540 & 1,314 & 78,188 & 0.38 & 0.34 \\
\hline July $\ldots \ldots . . . \ldots$ & 5,485 & 885 & 2,493 & 153,290 & 0.75 & 0.65 \\
\hline August $\ldots \ldots \ldots \ldots$ & 25,610 & 1,920 & 7,418 & 456,118 & 2.22 & 1.93 \\
\hline September $\ldots . . . .$. & 6,520 & 1,345 & 2,637 & 156,912 & 0.77 & 0.69 \\
\hline October $\ldots \ldots \ldots \ldots$ & 36,420 & 1,115 & 7,280 & 447,633 & 2.19 & 1.90 \\
\hline November ......... & 16,180 & 2,035 & 6,049 & 359,940 & 1.76 & 1.58 \\
\hline $\begin{array}{l}\text { December } \ldots . \cdots \\
1899 \text {. }\end{array}$ & 11,120 & 3,530 & 5,741 & 353,003 & 1.73 & 1.50 \\
\hline January ...... & 22,197 & 4,116 & 8,417 & 517,541 & 2.53 & 2.19 \\
\hline February $\ldots . . \ldots$ & 44,952 & 6,945 & 15,688 & 871,267 & 4.26 & 4.09 \\
\hline March $\ldots \ldots \ldots \ldots$ & 32,652 & 7,314 & 12,399 & 762,385 & 3.72 & 3.23 \\
\hline April $\ldots \ldots \ldots \ldots$ & 21,582 & 5,100 & 9,016 & 536,489 & 2.62 & 2.35 \\
\hline May $\ldots \ldots \ldots \ldots$ & 4,731 & 2,517 & 3,351 & 206,045 & 1.00 & 0.87 \\
\hline June $\ldots \ldots . . . .$. & 2,999 & 1,287 & 2,040 & 121,388 & 0.59 & 0.53 \\
\hline July ........... & 20,290 & 795 & 4,985 & 306,516 & 1.50 & 1.30 \\
\hline August ........... & 4,362 & 1,287 & 2,222 & 136,625 & 0.67 & 0.58 \\
\hline September .... & 2,394 & 430 & 984 & 58,552 & 0.29 & 0.26 \\
\hline October ....... & 1,656 & 320 & 1,014 & 62,348 & 0.30 & 0.26 \\
\hline November ......... & 7,068 & 918 & 1,787 & 106,334 & 0.53 & 0.47 \\
\hline $\begin{array}{l}\text { December } \ldots . \cdots \\
1900 \text {. }\end{array}$ & 18,138 & 1,656 & 4,728 & 290,713 & 1.42 & 1.23 \\
\hline January ......... & 10,335 & 2,125 & 3,728 & 229,226 & 1.12 & 0.97 \\
\hline February $\ldots . . .$. & 48,305 & 2,237 & 12,950 & 719,206 & 3.50 & 3.37 \\
\hline March $\ldots \ldots \ldots$ & 17,650 & 5,723 & 10,208 & 627,665 & 3.07 & 2.66 \\
\hline April $\ldots \ldots \ldots \ldots$ & 18,775 & 4,712 & 9,016 & 536,489 & 2.62 & 2.35 \\
\hline May $\ldots \ldots \ldots \ldots$ & 6,736 & 2,462 & 3,718 & 228,611 & 1.12 & 0.97 \\
\hline June $\ldots . . . . . . .$. & 27,831 & 2,575 & 8,317 & 494,896 & 2.42 & 2.17 \\
\hline July $\ldots \ldots \ldots \ldots \ldots$ & 11,572 & 2,462 & 5,405 & 332,340 & 1.63 & 1.41 \\
\hline August $\ldots \ldots \ldots \ldots$ & 6,960 & 1,675 & 2,814 & 173,026 & 0.84 & 0.73 \\
\hline September $\ldots . . . . .$. & 28,447 & 1,337 & 4,975 & 296,033 & 1.45 & 1.30 \\
\hline October ............. & 13,262 & 1,337 & 3,787 & 232,854 & 1.14 & 0.99 \\
\hline November $\ldots . . .$. & 11,460 & 2,462 & 4,224 & 251,345 & 1.23 & 1.10 \\
\hline December $\ldots . . .$. & 18,775 & 2,800 & 6,475 & 398,132 & 1.95 & 1.69 \\
\hline The year $\ldots \ldots \ldots$ & 48,305 & 1,337 & 6,301 & $4,519,823$ & 22.09 & 1.64 \\
\hline
\end{tabular}

*See explanation page 13. 
Estimated monthly discharge of Tallapoosa River near Milstead, Ala.

[Drainage area, 3,840 square miles.]

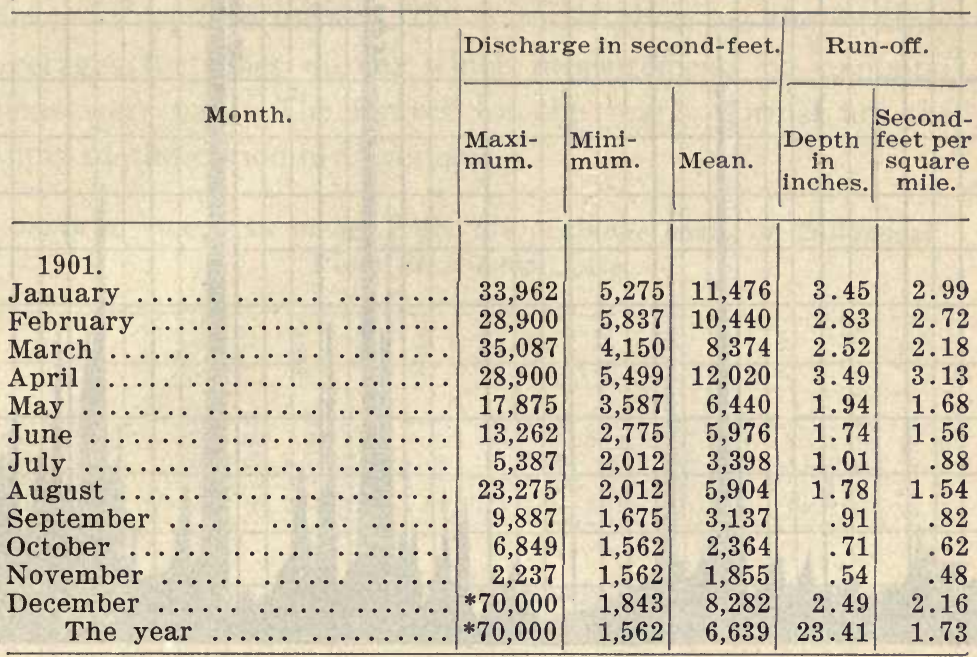

*Approximate.

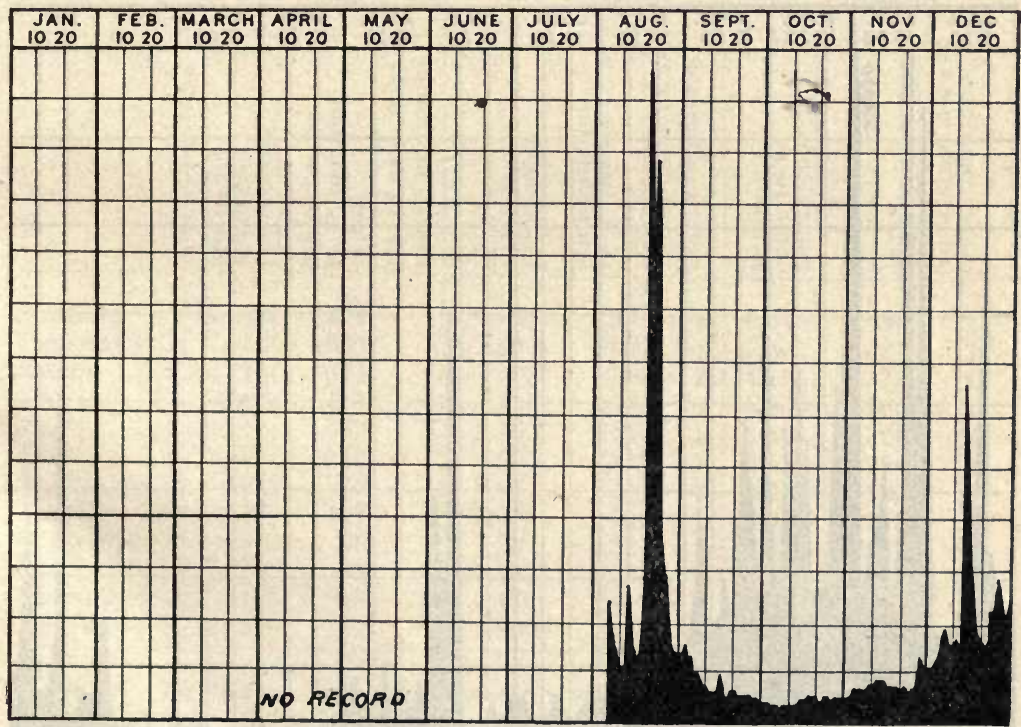

Fig. 1-Discharge of Tallapoosa River at Milstead, Ala., 1897. 


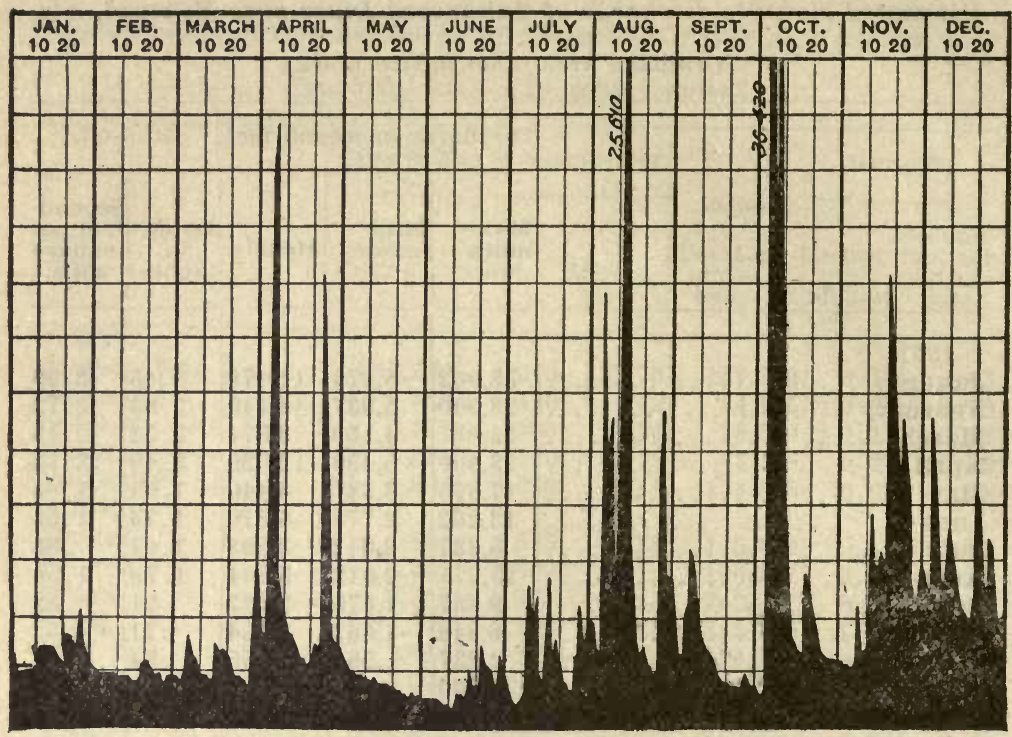

Fig. 2-Discharge of Tallapoosa River at Milstead, Ala., 1898.

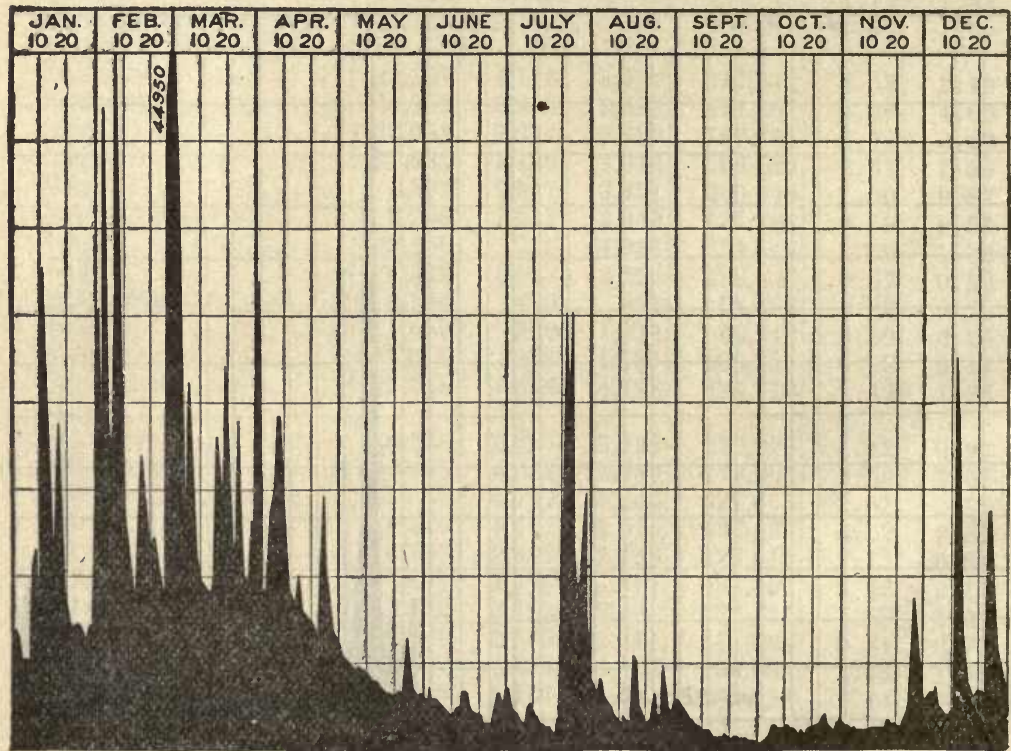

Fig. 3-Discharge of Tallapoosa River at Milstead, Ala.. 1899. 
The amount of water delivered from the drainage basin as measured at the points named below has been computed in terms of depth in inches. The normals given are the monthly averages for times during which measurements or computations were had. The figures for the yearly normal are the sums of these monthly averages.

Depth of run-off in inches from the drainage basin of Tallapoosa River at Milstead, Ala.

\begin{tabular}{|c|c|c|c|c|c|c|c|c|c|c|c|c|c|}
\hline Day. & ख્ & $\stackrel{0}{0}$ & $\sum_{\pi}^{\pi}$ & $\frac{\bar{\alpha}}{4}$ & $\stackrel{\vec{\pi}}{\overbrace{i}^{2}}$ & $\Xi$ & $\stackrel{3}{3}$ & $\frac{60}{3}$ & $\begin{array}{l}\vec{a} \\
\stackrel{a}{0} \\
\ddot{2}\end{array}$ & $\dot{\circlearrowright}$ & $\begin{array}{l}\dot{\sigma} \\
z\end{array}$ & 迎 & 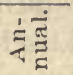 \\
\hline $\begin{array}{l}\text { Normal ... } \\
1895 \\
1899 \ldots \ldots \ldots \\
1990 \\
1901\end{array}$ & $\begin{array}{l}1.96 \\
.72 \\
2.53 \\
1.12 \\
3.45\end{array}$ & $\begin{array}{l}2.78 \\
.52 \\
4.26 \\
3.50 \\
2.83\end{array}$ & $\begin{array}{l}2.50 \\
.69 \\
3.72 \\
3.07 \\
2.52\end{array}$ & $\begin{array}{l}2.60 \\
1.67 \\
2.62 \\
2.62 \\
3.49\end{array}$ & $\begin{array}{l}1.15 \\
.45 \\
1.00 \\
1.12 \\
1.94\end{array}$ & $\begin{array}{r}1.28 \\
.38 \\
.59 \\
2.42 \\
1.74\end{array}$ & $\begin{array}{r}1.22 \\
.75 \\
1.50 \\
1.63 \\
1.01\end{array}$ & $\begin{array}{r}1.38 \\
2.22 \\
.67 \\
.84 \\
1.78\end{array}$ & $\begin{array}{r}.86 \\
.77 \\
.29 \\
1.45 \\
.91\end{array}$ & $\begin{array}{r}1 .(9 \\
2.19 \\
.30 \\
1.14 \\
.71\end{array}$ & \begin{tabular}{r|}
1.02 \\
1.76 \\
.53 \\
1.23 \\
.54
\end{tabular} & $\begin{array}{l}1.90 \\
1.73 \\
1.42 \\
1.95 \\
2.49\end{array}$ & $\begin{array}{l}19.70 \\
13.85 \\
19.43 \\
22.09 \\
23.41\end{array}$ \\
\hline
\end{tabular}

Minimum monthly discharge of Tallapoosa River at Milstead, Ala., with corresponding net horsepower per foot of fall on a water wheel realizing 80 per cent. of the theoretical power.

\begin{tabular}{|c|c|c|c|c|c|c|c|c|c|}
\hline & \multicolumn{3}{|c|}{1899} & \multicolumn{3}{|c|}{1900} & \multicolumn{3}{|c|}{1901} \\
\hline & 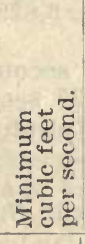 & 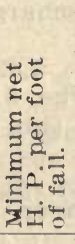 & 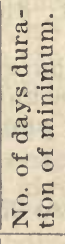 & 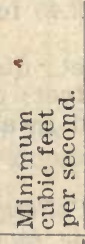 & 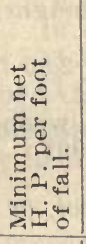 & 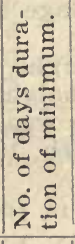 & 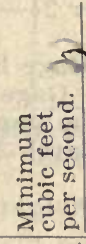 & 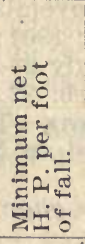 & 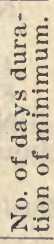 \\
\hline January & 4,116 & 374 & 1 & 2,125 & 193 & 4 & 5,275 & 480 & 1 \\
\hline February . & 6,945 & 631 & 1 & $2,23 \%$ & 203 & 1 & 5,837 & 531 & 1 \\
\hline March .... & 7,314 & 665 & 1 & 5,725 & 520 & 2 & 4,150 & 377 & 1 \\
\hline April $\ldots$. & 5,100 & 464 & 1 & 4,712 & 428 & 1 & 5,500 & 500 & 1 \\
\hline May ...... & 2,517 & 229 & 3 & 2,462 & 224 & 1 & 3,587 & 326 & 2 \\
\hline June ....... & 1,287 & 117 & 4 & 2,575 & 234 & 1 & 2,575 & 234 & 2 \\
\hline July ..... & 795 & 72 & 1 & 2,462 & 224 & 2 & 2,012 & 183 & 1 \\
\hline August ... & 1,287 & 117 & 3 & 1,675 & 152 & 1 & 2,012 & 183 & 5 \\
\hline September & 430 & 39 & 3 & 1,337 & 122 & 2 & 1,675 & 152 & 2 \\
\hline October ... & 320 & 29 & 2 & 1,337 & 122 & 1 & 1,562 & 142 & 10 \\
\hline November & 918 & 83 & 5 & 2,462 & 244 & 9 & 1,562 & 142 & 3 \\
\hline December & 1,656 & 151 & 1 & 2,800 & 255 & 3 & 1,843 & 168 & 1 \\
\hline
\end{tabular}

Note-To find the minimum net horse power available at a shoal on this stream, near this station, for any month, multiply the total fall of the shoal by the "net H. P. per foot of fall" in this table for that month. 


\section{TALI.APOOSA RIVER NEAR SUSANNA, ALABAMA.}

This station was established July 27 , I900, by J. R. Hall. It is located at the mouth of Blue Creek, which is Io feet above the east landing of McCarty's ferry, I3 miles southwest of Dadeville, and 3 miles from Susanna, the nearest postoffice. The rod is graduated to feet and tenths; it is 18 feet long, and is nailed vertically to a tree overhanging the water on the south side of the creek at the junction of the creek and the river. The gage is referred to a bench mark on a white hickory tree about 40 feet from the rod on the south bank of the creek, and is 376.67 feet above tide water. Discharge measurements are made from a boat held in place by a wire stretched across the river, upon which the distances from the initial point are tagged. The section is an exceptionally good one, depth and current being almost uniform the entire width of the stream. The observer is T. A. Walls, a farmer who lives I mile from the station. During 1900 and I90I the following discharge measurements were made by James R. Hall:

1900:

July 27-Gage height, 1.80 feet; discharge, 2,309 second-feet.

August 9 -Gage height, 1.55 feet; discharge, 1,900 second-feet.

September 28-Gage height, 1.50 feet; discharge, 1,809 second-feet.

November 24-Gage height, 2.40 feet; discharge, 3,629 second-feet. 1901:

July 9 -Gage height, 2.80 feet; discharge, 5,628 second-feet.

Feb. 27.-Gage height, 2.90 feet; discharge, 5,135 second-feet.

Note-The gage was washed away, and this station was discontinued on March 30th, 1901.

Daily gage height, in feet, of Tallapoosa River, near Susanna, Ala., for 1900.

\begin{tabular}{|c|c|c|c|c|c|c|c|c|c|c|c|c|c|}
\hline Day. & $\stackrel{3}{\xi}$ & $\stackrel{60}{4}$ & $\begin{array}{l}\overrightarrow{0} \\
\overrightarrow{0}_{2} \\
{ }_{2}\end{array}$ & ப் & $\begin{array}{l}\vec{b} \\
\dot{z}\end{array}$ & $\begin{array}{l}\dot{\Xi} \\
\stackrel{\Phi}{ }\end{array}$ & Day. & $\stackrel{\overrightarrow{3}}{3}$ & $\begin{array}{l}80 \\
3 \\
4\end{array}$ & $\begin{array}{l}\overrightarrow{0} \\
\stackrel{0}{0} \\
02\end{array}$ & $\dot{0}$ & $\begin{array}{l}3 \\
\text { z }\end{array}$ & $\begin{array}{l}\dot{\delta} \\
\stackrel{\Phi}{ }\end{array}$ \\
\hline & & $\begin{array}{l}5.80 \\
4.00 \\
2.00 \\
1.80 \\
1.80 \\
2.10 \\
2.20 \\
1.70 \\
1.55 \\
1.50 \\
1.40 \\
1.40 \\
1.40 \\
1.40 \mid \\
1.40 \mid \\
1.90 \mid 1\end{array}$ & $\begin{array}{l}2.40 \\
3.80 \\
4.80 \\
4.20 \\
2.25 \\
1.50 \\
1.45 \\
1.45 \\
1.40 \\
1.35 \\
1.35 \\
1.35 \\
1.35 \\
1.30 \\
1.35 \\
1.70\end{array}$ & $\begin{array}{l}1.40 \\
1.40 \\
1.40 \\
1.35 \\
1.30 \\
2.80 \\
3.00 \\
2.50 \\
1.85 \\
1.80 \\
1.75 \\
1.70 \\
1.90 \\
2.40 \\
2.45 \\
2.40\end{array}$ & $\begin{array}{l}1.80 \\
1.70 \\
1.70 \\
1.65 \\
1.65 \\
1.65 \\
1.60 \\
1.60 \\
1.60 \\
1.60 \\
1.60 \\
1.55 \\
1.55 \\
1.60 \\
1.6 \mathrm{~b}\end{array}$ & $\begin{array}{l}2.00 \\
2.00 \\
2.10 \\
2.20 \\
2.30 \\
2.50 \\
2.40 \\
2.40 \\
2.40 \\
2.30 \\
2.10 \\
2.00 \\
1.90 \\
3.90 \\
3.90 \\
2.80\end{array}$ & $\begin{array}{l}21 \\
2 \\
2 \\
2 \\
25\end{array}$ & 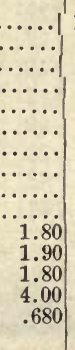 & $\begin{array}{l}1.95 \\
1.95 \\
1.80 \\
1.75 \\
1.70 \\
1.70 \\
1.90 \\
2.00 \\
2.05 \\
2.50 \\
2.15 \\
2.00 \\
1.90 \\
1.80 \\
2.25\end{array}$ & $\begin{array}{l}8.40 \\
4.80 \\
3.00 \\
2.50 \\
1.80 \\
1.80 \\
1.80 \\
1.70 \\
1.60 \\
1.50 \\
1.50 \\
1.50 \\
1.45 \\
1.45 \\
\ldots . .\end{array}$ & $\begin{array}{l}2.35 \\
2.30 \\
2.20 \\
2.10 \\
1.90 \\
1.70 \\
3.90 \\
6.00 \\
5.00 \\
4.30 \\
4.10 \\
2.30 \\
2.20 \\
1.90 \\
1.85\end{array}$ & $\begin{array}{l}1.75 \\
1.75 \\
1.80 \\
1.85 \\
1.85 \\
1.90 \\
2.40 \\
2.40 \\
3.00 \\
4.90 \\
4.20 \\
3.90 \\
3.00 \\
2.80 \\
\ldots . .\end{array}$ & $\begin{array}{l}4 \\
4 \\
3 \\
2 \\
2\end{array}$ \\
\hline
\end{tabular}


Daily gage height of Tallapoosa River at Susanna, Ala., for 1901.

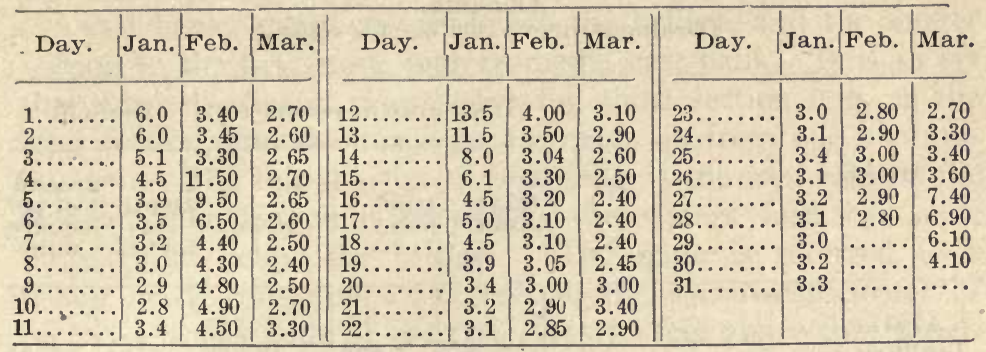

Rating table for Tallapoosa River, at Susanna, Ala., 1900 and 1901.

\begin{tabular}{c|c|c|c}
\hline Gage Height. & Discharge. & Gage Height. & Discharge. \\
\hline 1.0 & $\ldots \ldots$ & 4.0 & 11,030 \\
1.2 & $\ldots \ldots 3$ & 4.2 & 11,930 \\
1.4 & 1,680 & 4.4 & 12,830 \\
1.6 & 1,960 & 4.6 & 13,730 \\
1.8 & 2,320 & 4.8 & 14,630 \\
2.0 & 2,740 & 5.0 & 15,530 \\
2.2 & 3,230 & 5.5 & 17,780 \\
2.4 & 3,850 & 6.0 & 20,030 \\
2.6 & 4,730 & 6.5 & 22,280 \\
2.8 & 5,630 & 7.0 & 24,530 \\
3.0 & 6,530 & 8.0 & 29,030 \\
3.2 & 7,430 & 9.0 & 33,530 \\
3.4 & 8,330 & 10.0 & 38,030 \\
3.6 & 9,230 & 11.0 & 42,530 \\
3.8 & 10,130 & 11.7 & 45,680 \\
\hline
\end{tabular}

Note-This table applied to the foregoing "Daily gage heights" gives the cubic feet per second flowing in the river on each date for which the gage height is given.

Estimated monthly discharge of Tallapoosa River, near Susanna, Alabama.

[Drainage area, 2,610 square miles.]

\begin{tabular}{|c|c|c|c|c|c|c|}
\hline \multirow[b]{2}{*}{ Month } & \multicolumn{3}{|c|}{ Discharge in second-feet. } & \multirow[b]{2}{*}{$\begin{array}{l}\text { Total in } \\
\text { acre- } \\
\text { feet. }\end{array}$} & \multicolumn{2}{|c|}{ Run-off. } \\
\hline & $\begin{array}{l}\text { Maxi- } \\
\text { mum. }\end{array}$ & $\begin{array}{l}\text { Mini- } \\
\text { mum. }\end{array}$ & Mean. & & $\begin{array}{l}\text { Depth } \\
\text { in } \\
\text { inches. }\end{array}$ & $\begin{array}{l}\text { Second } \\
\text { feet per } \\
\text { square } \\
\text { mile. }\end{array}$ \\
\hline $\begin{array}{l}1900 \\
\text { July } 27 \text { to } 31 \ldots \ldots\end{array}$ & & & 8,364 & & & \\
\hline August $\ldots \ldots \ldots$. . & 19,130 & 1,680 & 3,258 & 200,327 & 1.44 & 1.25 \\
\hline September ......... & 45,680 & 1,570 & 6,083 & 361,964 & 2.60 & 2.33 \\
\hline October $\ldots \ldots \ldots \ldots$ & 20,030 & 1,570 & 4,776 & 293,665 & 2.11 & 1.83 \\
\hline November .......... & 15,080 & 1,885 & 3,676 & 218,737 & 1.57 & 1.41 \\
\hline December ......... & 19,130 & 2,520 & 6,288 & 386,634 & 2.78 & 2.41 \\
\hline
\end{tabular}


Estimated monthly discharge of Tallapoosa River, near Susanna, Alabama.

[Drainage area, 2,610 square miles.]

\begin{tabular}{|c|c|c|c|c|c|}
\hline \multirow[b]{2}{*}{ Month. } & \multicolumn{3}{|c|}{ Discharge in second-feet } & \multicolumn{2}{|c|}{ Run-off. } \\
\hline & $\begin{array}{l}\text { Maxi- } \\
\text { mum. }\end{array}$ & $\begin{array}{l}\text { Mini- } \\
\text { mum. }\end{array}$ & Mean. & $\begin{array}{l}\text { Depth } \\
\text { in } \\
\text { inches. }\end{array}$ & $\begin{array}{l}\text { Second. } \\
\text { feet per } \\
\text { square } \\
\text { mile. }\end{array}$ \\
\hline 1901. & & & & & - \\
\hline January $\quad \ldots \ldots \ldots \ldots \ldots \ldots$ & 53,780 & 5,630 & 13,265 & 5.86 & 5.08 \\
\hline February $\ldots \ldots \ldots \ldots \ldots \ldots$ & 44,780 & 5,630 & 11,303 & 4.51 & 4.33 \\
\hline March...$\ldots \ldots \ldots \ldots \ldots$ & 26,330 & 3,850 & 7,546 & 3.31 & 2.89 \\
\hline
\end{tabular}

Minimum monthly discharge of Tallapoosa River at Susanna, Ala,, with corresponding net horsepower per foot of fall on a water wheel realizing 8 (I) per cent. of the theoretical power.

[Drainage area, 2,610 square miles.]

i

\begin{tabular}{|c|c|c|c|c|c|c|c|}
\hline & \multicolumn{3}{|c|}{1900} & & \multicolumn{3}{|c|}{1901} \\
\hline & 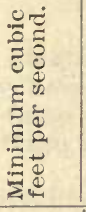 & 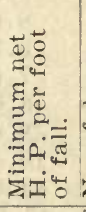 & & & 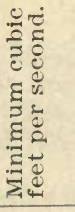 & 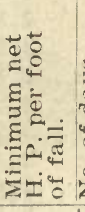 & 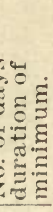 \\
\hline July & 2,320 & 211 & 2 & January & 5,630 & 512 & \\
\hline August .. & 1,680 & 153 & 5 & February ... & 5,630 & 512 & \\
\hline September & 1,570 & 143 & 1 & March ..... & 3,850 & 350 & \\
\hline October ...... & 1,570 & 143 & 1 & & & & \\
\hline November .... & 1,885 & 171 & 2 & & & & \\
\hline December .... & 2,520 & 229 & 1 & & & & \\
\hline
\end{tabular}

Note-To find the minimum net horse power available at a shoal on this stream, near this station, for any month, multiply the total fall of the shoal by the "net H. P. per foot of fall" in this table for that month.

3. TALTAPOOSA RIVER, NEAR STURDEVANT, ALABAMA.

This station was established July I9, I900, by J. R. Hall. It is located at the bridge, Columbus \& Western Division of the Central of Georgia Railroad, a fourth of a mile west of Sturdevant. The gage rod is 20 feet high, and is graduated to feet and tenths. It is in two sections, and is fastened verti- 
cally, the shorter section to a post at the edge of the waer on the east bank, about 20 feet below the bridge, and the longer section to the first stone pier from the east bank. It is so set that when the water rises above the short section it is on the long section, and the readings are made as from one continuous rod. The initial point of sounding is the east end of the bridge. The section is broken by three piers and by scme large rocks below the bridge. The gage is referred to a bench mark consisting of a nail in the southwest corner of pier No. 2 , east side of the river, 455.70 feet above tide water, and 14.20 feet above the zero of the gage. The sbserver is B. F. Neighbors, farmer and postmaster at Sturdevant, who lives a fourth of a mile from the station. During Igoo the iollowing discharge measurements were made by James $R$. Hall :

July 20-Gage height; 2.85 feet; discharge, 2,603 second-feet.

August 13-Gage height, 1.95 feet; discharge, 1,887 second-feet.

Daily gage height, in feet, of Tallapoosa River near sturdevant, Ala. for 1900 .

\begin{tabular}{|c|c|c|c|c|c|c|c|c|c|c|c|c|c|}
\hline & uly & Aug: & Sept & Oet $\mid$ & Novil & Dec & Day. & July & $\mathrm{ug} / \mathrm{s}$ & Sept & Oct & Nov & De \\
\hline & & $\begin{array}{l}4.30 \\
3.40 \\
2.80 \\
2.50 \\
2.40 \\
2.35 \\
2.25 \\
2.20 \\
2.16 \\
2.00 \\
1.95 \\
1.90 \\
2.00 \\
2.10 \\
2.60 \\
3.40\end{array}$ & \begin{tabular}{|c|}
$3.40 \mid$ \\
6.10 \\
4.20 \\
$2.90 \mid$ \\
$2.50 \mid$ \\
$2.20 \mid$ \\
$2.00 \mid$ \\
$1.90 \mid$ \\
1.80 \\
1.70 \\
$1.60 \mid$ \\
1.60 \\
1.60 \\
1.80 \\
$8.80 \mid$ \\
12.00
\end{tabular} & \begin{tabular}{|}
1.80 \\
1.70 \\
1.60 \\
$1.60 \mid$ \\
3.30 \\
$3.00 \mid$ \\
$3.00 \mid$ \\
2.90 \\
3.10 \\
3.20 \\
$3.30 \mid$ \\
3.40 \\
3.50 \\
$3.50 \mid$ \\
3.00 \\
2.60
\end{tabular} & \begin{tabular}{|l|}
$2.50 \mid$ \\
$3.40 \mid$ \\
$4.70 \mid$ \\
$3.60 \mid$ \\
$3.30 \mid$ \\
$3.20 \mid$ \\
$2.90 \mid$ \\
$2.70 \mid$ \\
$2.60 \mid$ \\
$2.50 \mid$ \\
$2.40 \mid$ \\
$2.40 \mid$ \\
$2.40 \mid$ \\
$2.30 \mid$ \\
$2.30 \mid$ \\
$2.30 \mid$
\end{tabular} & $\begin{array}{l}2.90 \\
2.80 \\
2.70 \\
3.00 \\
3.20 \\
3.30 \\
3.20 \\
3.00 \\
2.80 \\
2.70 \\
2.60 \\
2.60 \\
2.70 \\
4.70 \\
3.00 \\
3.70\end{array}$ & $\begin{array}{l}18 \ldots \ldots \\
19 \ldots \\
20 \ldots \\
21 \ldots \\
22 \ldots \\
23 \ldots \\
24 \ldots \\
25 \ldots \\
26 \ldots \\
27 \ldots \\
23 \ldots \\
29 \ldots \\
30 \ldots \\
31 \ldots\end{array}$ & \begin{tabular}{r|}
2.95 \\
2.80 \\
3.05 \\
2.75 \\
2.65 \\
2.55 \\
2.65 \\
2.60 \\
2.50 \\
2.70 \\
6.50 \\
7.60 \\
5.00
\end{tabular} & $\mid \begin{array}{l}3 . \mathrm{c} \\
2.80 \\
4.00 \\
2.90 \\
2.40 \\
2.40 \\
2.30 \\
2.40 \\
2.70 \\
2.50 \\
2.80 \\
2.60 \mid \\
2.50 \\
2.40 \\
2.30 \\
2.80\end{array}$ & 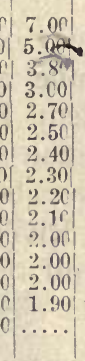 & $\begin{array}{r}2.6 f \\
2.20 \\
2.10 \\
2.00 \\
1.90 \\
2.10 \\
5 . f 5 \\
7.30 \\
6.40 \\
5.4 ! \\
4.30 \\
3.60 \\
2.90 \\
2.7 f \\
2.60\end{array}$ & $\mid \begin{array}{l}2.30 \\
2.20 \\
2.20 \\
2.20 \\
2.50 \\
3.20 \\
3.50 \\
3.20 \\
4.80 \\
5.90 \\
5.40 \\
3.40 \\
3.20 \\
3.10 \\
\ldots \ldots\end{array}$ & 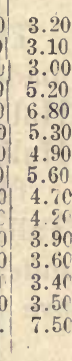 \\
\hline
\end{tabular}

During the year rgor James R. Hall made one measurement, as follows:

March 8-Gage height, 3.40 feet; discharge, 3,774 second-feet.

During the year 1902 the following discharge measurements have been made at Sturdevant by W. E. Hall:

July 11-Gage height, 1.85 feet; discharge, 1,440 second-feet.

September 17 -Gage height, 0.80 feet; discharge, 658 second-feet. October 9-Gage height. 1.08 feet; discharge, 858 second-feet.

November 12-Gage height, 134 feet; discharge, 1,000 second-feet. 
Daily gage height of Tallapoosa River at Sturdevant, Ala., for 1901.

\begin{tabular}{r|r|r|r|r|r|r|r|r|r|r|r|r}
\hline \multicolumn{2}{r|}{ Day. Jan. } & Feb. & Mar. & April & May & June & July & Aug. & Sept. & Oct. & Nov. & Dec \\
& & &
\end{tabular}

4. SURVEY OF TALLAPOOSA RIVER IN AIABAMA.

This survey of a part of Tallapoosa River in Alabama was made in June and July, I9oo, under supervision of B. M. Hall, resident Hydrographer, by Field Engineer James R. Hall, levelman and topographer.

The survey began at the Hydrographic Station on the Tallapoosa River, at Milstead, Ala., and ran up the river 64 miles to head of shoal above Griffn's Ferry. The elevations are sea-level elevations.

\section{DESCRIPTION OF RIVER.}

The entire river above Milstead runs on granite bed-rock, and has numerous bluffs along its banks, forming excellent sites for dams.

There are two large developed water powers on the river:

- The Tallassee Falls plant, and the Montgomery Power Company's plant, both of which are near the lower end of the survey. (See Plates $B$ and $C$, opposite.) 

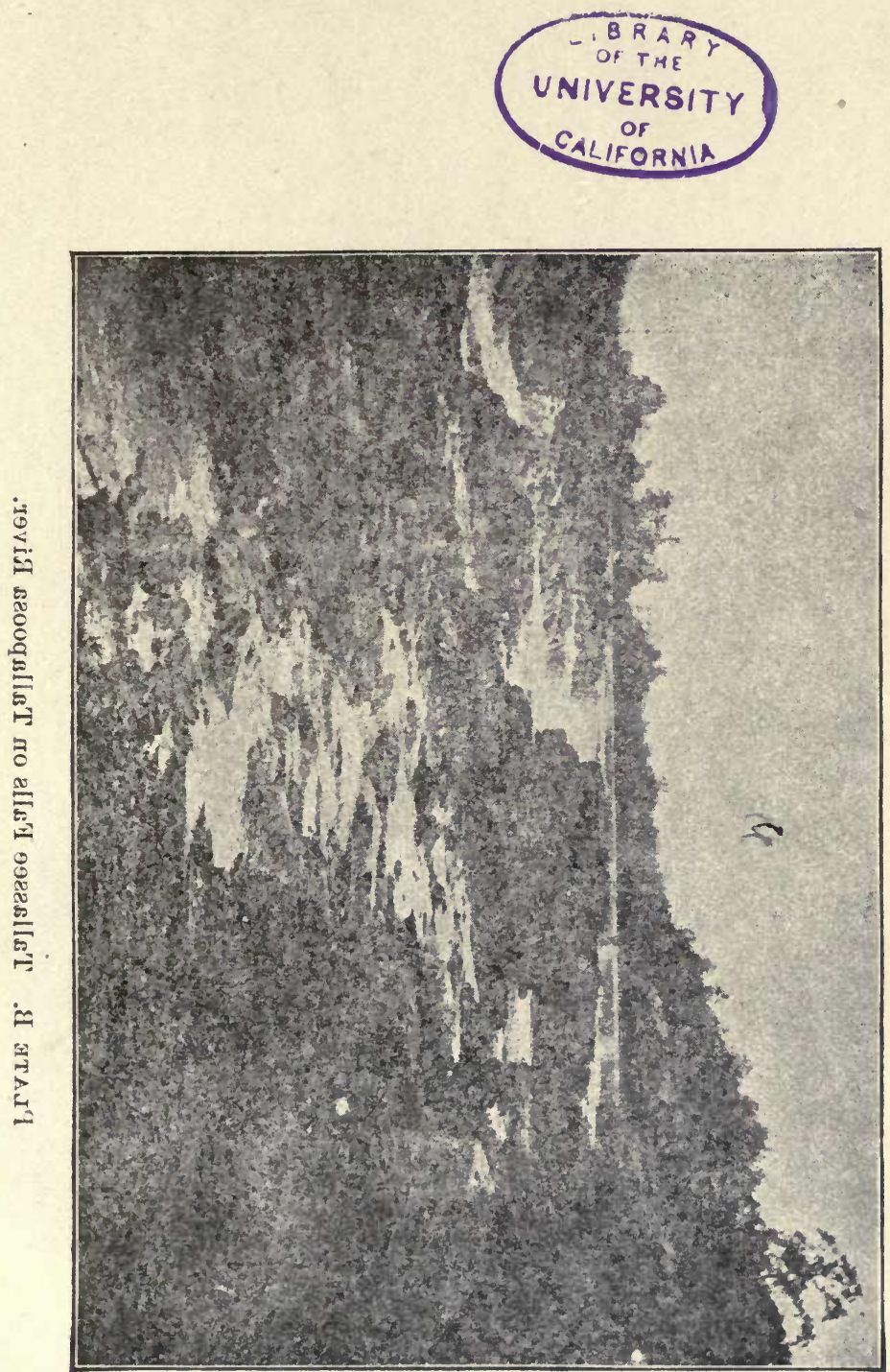


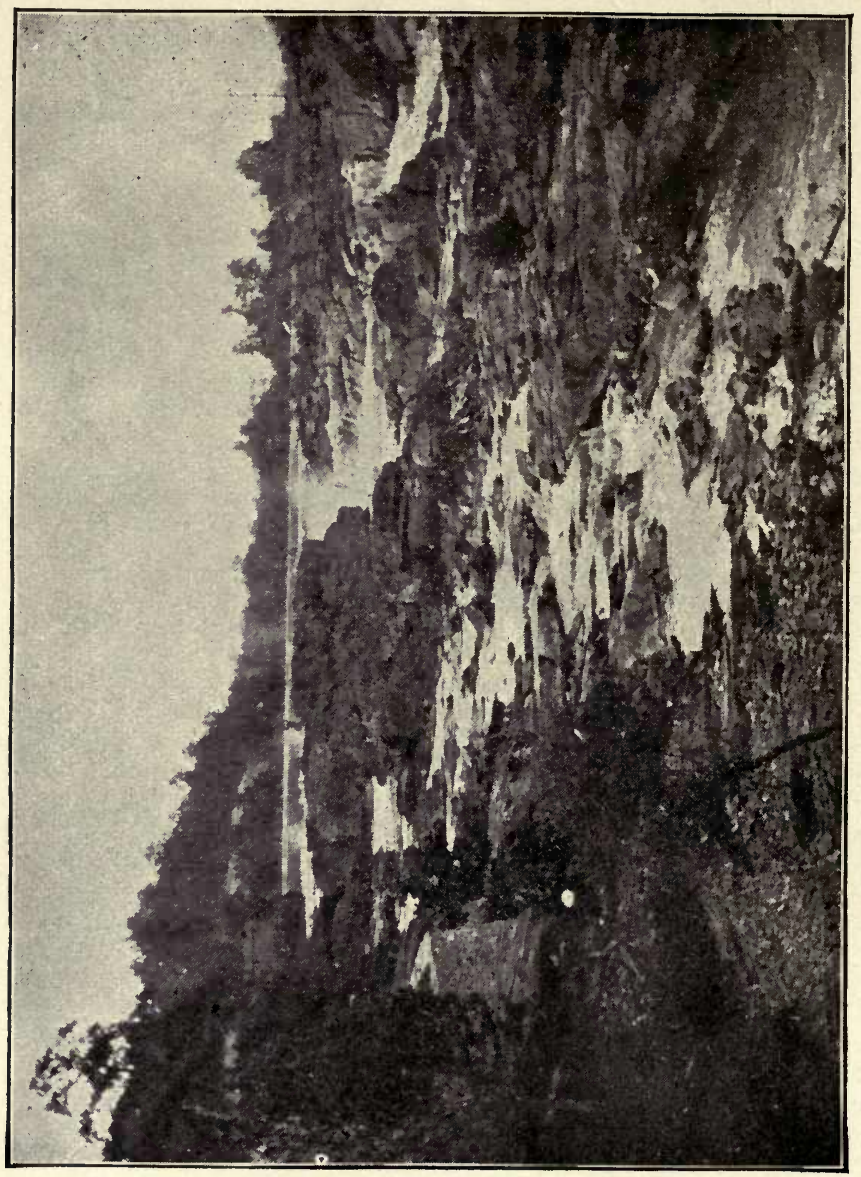

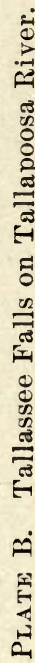





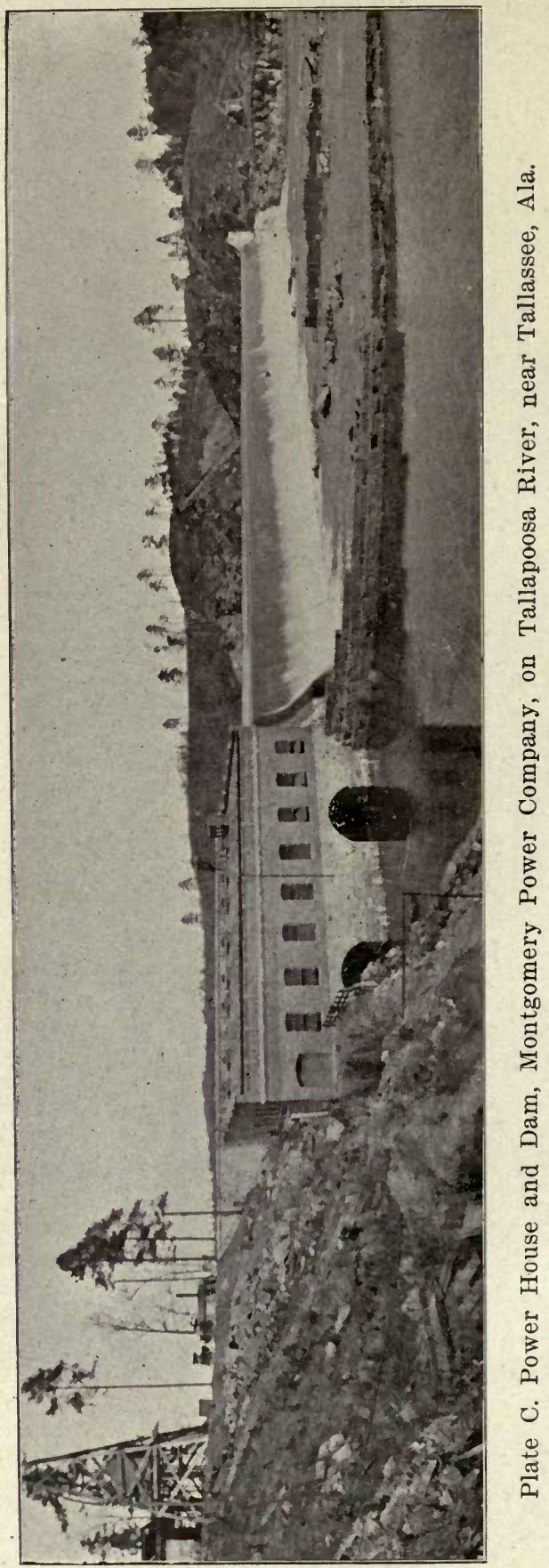


The Tallassee Falls dam and canal, which are six miles above Milstead, utilize a fall of 64 feet, with the whole river. This power and its large cotton manufacturing plant recently completed, is described in the Twentieth Annual Report, U. S. Geological Survey, Part IV, Pages 192-I93. This power was capable of realizing $\delta, 900$ net $\mathrm{H}$. P. without storage during low water of Uctober, I90I. A break which occurred in the dam on December 29, I90I, has decreased the present available head, but does not stop the machinery.

The Montgomery Power Company dam has a 40-foot dam, nine and a half miles abcve Milstead. It backs the water six and a half miles up the river, and forms an immense storage basin. This being almost completed in December, 1901, was partly washed out by a great flood December 29, I90I. The water wheels, dynamos, pole line and wiring to Montgomery are all installed, and ready for work as soon as the dam is repaired. The distance to Montgomery is about 27 miles.

With river at stage of lowest water during October, I90I, this plant will develop at the wheels 5.572 net $H$. P. from the run of the river without drawing on the storage.

The equalizing storage of this dam will add fully 25 per cent. to this power and to the power at Tallassee for continuous rumning without materially lowering the head at either plant.

The following list of distances and elevations of water and bench marks shows the fall of the river from point to point. The total fall in $5^{6}$ of the 64 miles surveyed is $36_{4}$ feet. 
Elevations and benciz marks along Tallapoosa River from Milstead, Ala., to Griffin Shoals.

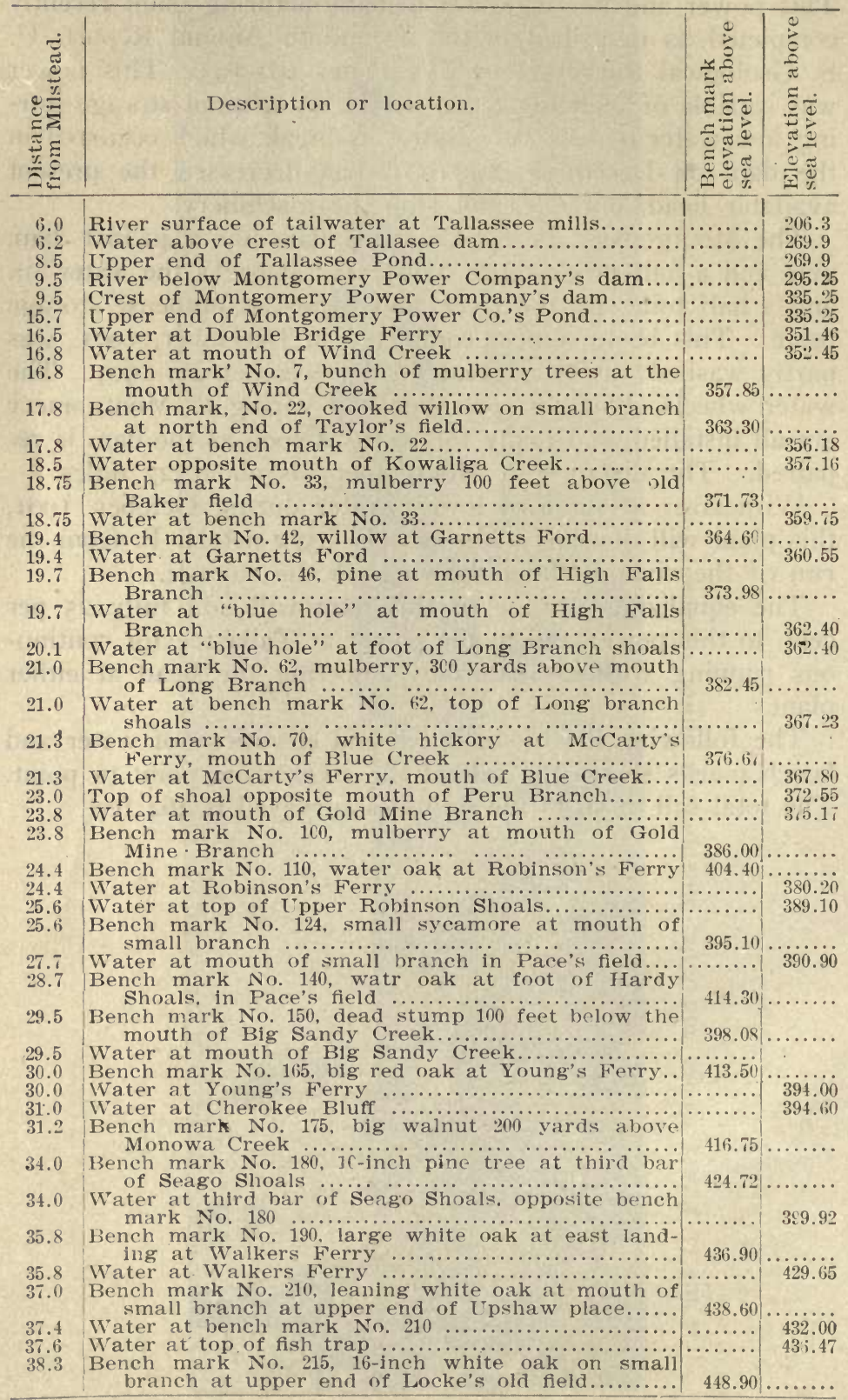




\begin{tabular}{|c|c|c|c|}
\hline 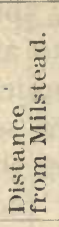 & Description or location. & 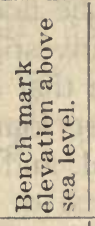 & 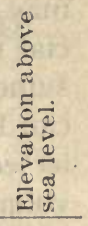 \\
\hline & Water at bench mark No. $215 \ldots \ldots \ldots \ldots$ & & 438.00 \\
\hline & $\begin{array}{l}\text { Water under Central railroad bridg at Sturdevant, } \\
\text { Alabama } \ldots \ldots \ldots \ldots \ldots \ldots \ldots \ldots \ldots \ldots \ldots \ldots \ldots \ldots \ldots \ldots \ldots \ldots \ldots\end{array}$ & & 444.25 \\
\hline 39 . & $\begin{array}{l}\text { Bench mark on top of rail over first pier of the } \\
\text { east end of Central Railroad bridge } \ldots \ldots \ldots \ldots \ldots \ldots\end{array}$ & 505.90 & \\
\hline 41. & $\begin{array}{c}\text { Bench mark No. } 20 \text {, large water oak at east land- } \\
\text { ing of Dennis Ferry } \ldots \ldots \ldots \ldots \ldots \ldots \ldots \ldots \ldots \ldots \ldots \ldots \ldots \ldots \ldots\end{array}$ & 457.15 & \\
\hline 41. & 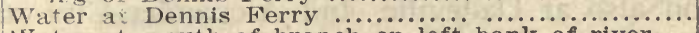 & & \\
\hline 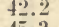 & Water at mouth of branch on left bank of river... & & \\
\hline 45. & Water 600 feet below mouth of Hillabee Creek........ & & 47 \\
\hline & $\begin{array}{l}\text { Bench mark No. } 310, \text { water oak at east landing of } \\
\text { Welch's Ferry } \ldots \ldots \ldots \ldots . \ldots \ldots \ldots\end{array}$ & 504.15 & \\
\hline 48. & 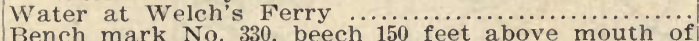 & & 492.30 \\
\hline & $\begin{array}{l}\text { Bench mark No. } 330 \text {, beech } 150 \text { feet above mouth of } \\
\text { Freeman's Branch } \ldots \ldots \ldots \ldots \ldots \ldots \ldots \ldots \ldots \ldots \ldots \ldots \ldots \ldots \ldots \ldots \ldots \ldots\end{array}$ & & \\
\hline 50.6 & Water 150 feet above mouth of Freeman's Branch.. & .... & \\
\hline $5 \%$. & Water at Whaleys Ferry $\ldots \ldots \ldots \ldots \ldots \ldots \ldots \ldots$ & & \\
\hline & $\begin{array}{l}\text { Bench mark No. } 340, \text { birch at } \\
\text { Bench mark No. } 350,10 \text {-inch birch at Millers Ferry.. }\end{array}$ & $\begin{array}{l}539.38 \\
552.16\end{array}$ & \\
\hline & 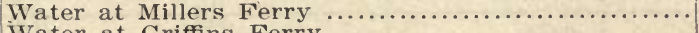 & & \\
\hline & 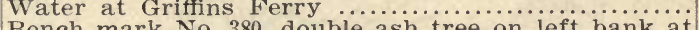 & & 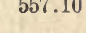 \\
\hline & $\begin{array}{l}\text { Bench mark No. } 380, \text { double ash tree on left bank at } \\
\text { Griffins Ferry } \ldots \ldots \ldots \ldots \ldots \ldots \ldots \ldots \ldots\end{array}$ & 564.76 & \\
\hline 62. & $\begin{array}{c}\text { Bench mark No. } 390,12-i n c h \text { birch at head of Griffins } \\
\text { Shoals } \ldots \ldots \ldots \ldots \ldots \ldots \ldots \ldots \ldots \ldots\end{array}$ & & \\
\hline 62.0 & Water at head of Griffins Shoals $\ldots \ldots \ldots \ldots \ldots \ldots \ldots . . . . .$. & & 570.30 \\
\hline
\end{tabular}

Surveys have been made for a large dam, 35 or 40 feet in height, at or near Double Bridge Ferry, to back the water beyond Robinson's Ferry, $z$ distance of about 8 pules up the river. There is an excellent site for a dam, and the project is entirely feasible. The horse power in proportion to head would be the same as that available at the Montgomery Power Company's dam.

From the mouth of Big Sandy Creek to a point one mile above Griffin's Ferry, a distance of 32 miles, the fall of the Tallapoosa River is 176.5 feet. Nearly all of this fall can be utilized for power by developments similar to those which have been made, and proprosed below. A study of the profile and of the above table of distances and elevations will give the distribution of the fali, showing the distance to which dams of certain height will back the water, at the various shoals, but the question of the best power sites, and the proper plan of development, height and location of dams, etc., for any point will depend on the special conditions favorable or unfavorable for dams and canals, the width of river bed, or flooded areas above, and the value of farming lands which 
may be flooded. All of which can be determined only by special investigation and surveys. It will be safe, however, to assume that a practicable site for a dam 40 feet high or under, can be found in the vicinity of any location which may be selected, and the power obtainable can be estimated by using the volume of water, or its equivalent net horse power per foot of fall, and the proposed head to be developed.

The water supply or discharge of Tallapoosa River at different points may be closely approximated from the foregoing records of Milstead, Susanna, and Sturdevant Hydrographic Stations, and also at Dadeville, and Alexander City stations, on the tributaries. 


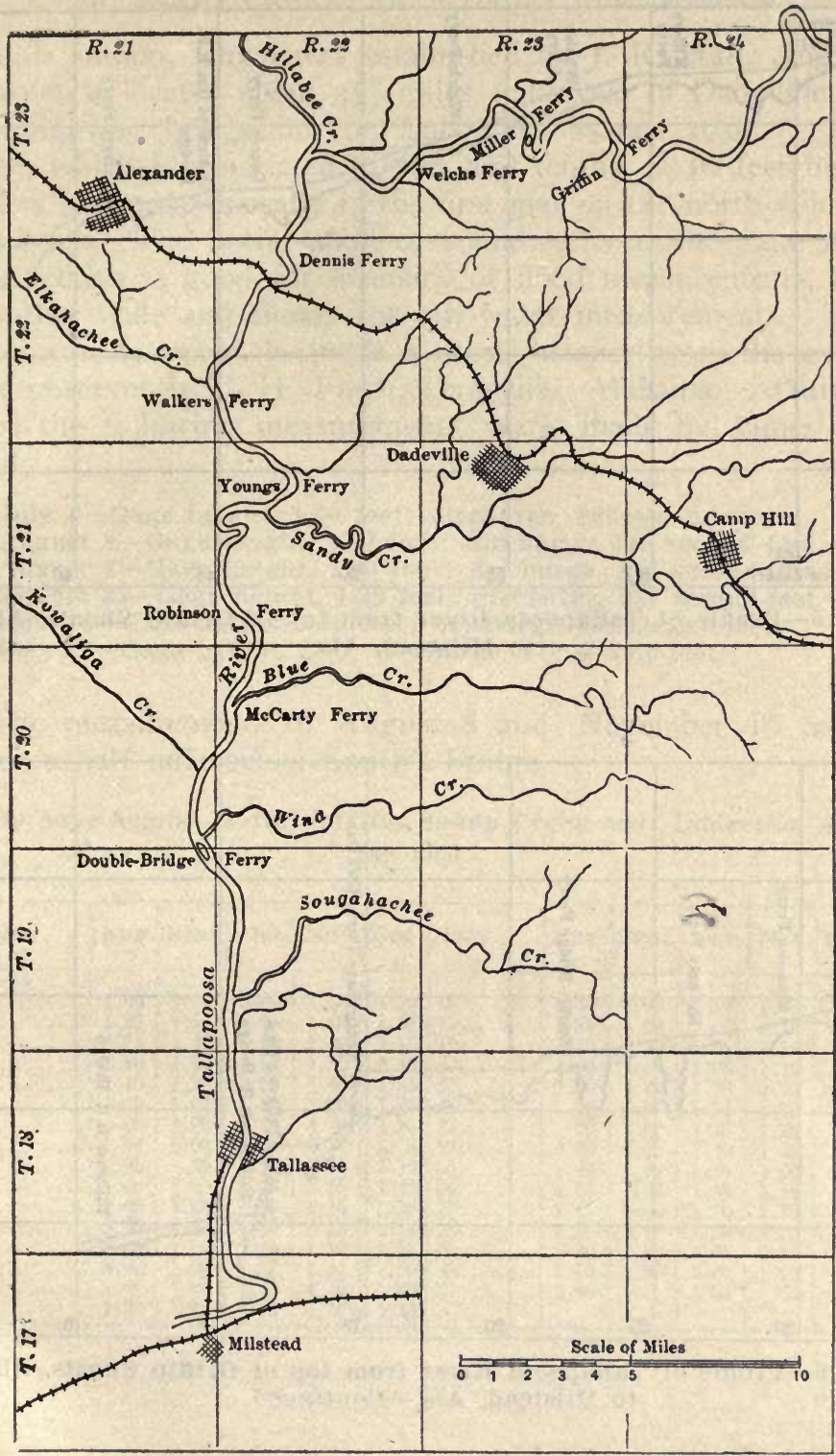

Fig. 4-Map of Tallapoosa River from top of Griffin Shoals, Ala., to Milstead, Ala. 


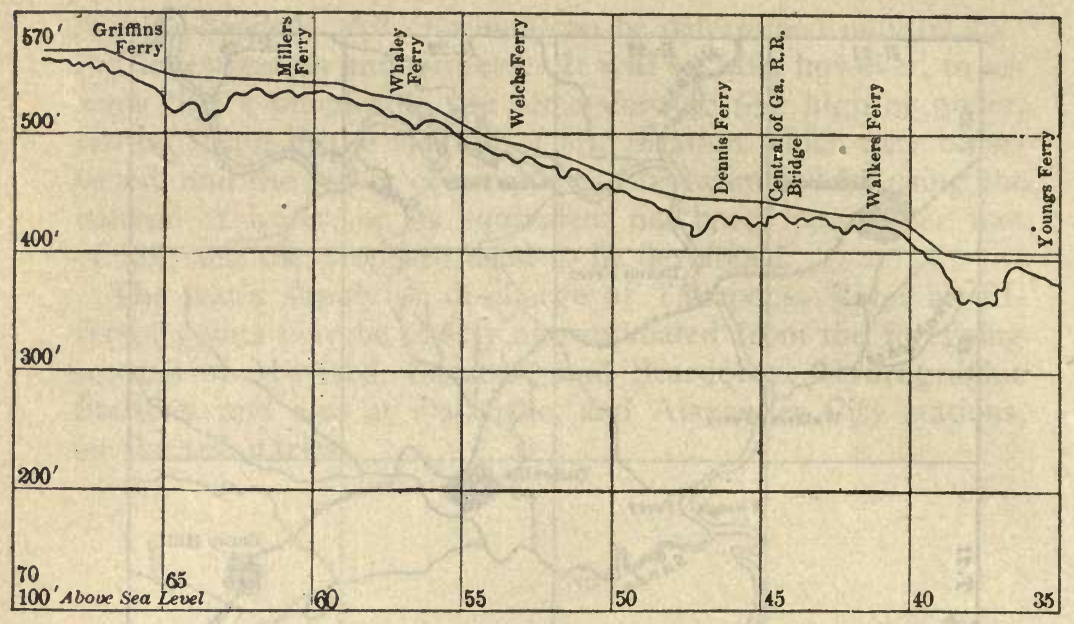

Fig. 5-Profile of Tallapoosa River from top of Griffin Shoals, Ala., to Milstead, Ala.

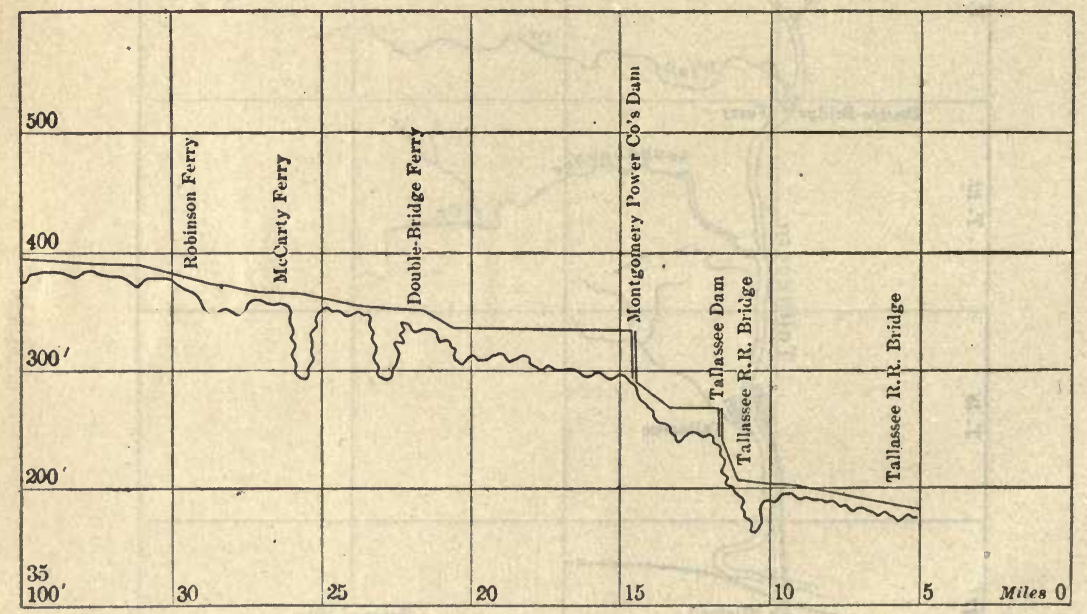

Fig. 6-Profile of Tallapoosa River from top of Griffin Shoals, Ala., to Milstead, Ala.-Continued. 
5. BIG SANDY CREEK. NEAR DADEVIILE, ALABAMA.

This station, which was established by J. R. Hall, August 2, I900, is located about $4^{1 / 2}$ miles southwest of Dadeville, at the highway bridge on the Dadeville-Susanna road. The gage, which is graduated to feet and tenths, is 16 feet high, and is fastened vertically to the first pier on the north side of the creek. The initial point of sounding is at the gage rod. The section is good for ordinary or flood measurements, but is rather wide and shoaly for $1 \mathrm{w}$-water measurements. The latter can, however, be made a short distance from the gage. The observer is T. H. Finch, Dadeville, Alabama. During Igoo the following measurements were made by James R. Hall :

July 6-Gage height, 1.20 feet; discharge, 260 second-feet.

August 8-Gage height, 1.00 foot; discharge, 110 second feet.

August 8-Gage height, 1.00 foot; discharge, 116 second-feet.

August 25-Gage height, 1.35 feet; discharge, 281 second-feet.

Nov. 16-Gage height, 1.10 feet; discharge, 155 second-feet.

Dec. 31 -Gage height, 2.00 ; discharge, 870 second-feet.

The measurements of August 8 and November if were inade a half mile below Smith's bridge.

Daily gage height, in feet, of Big Sandy Creek near Dadeville. Ala., for 1900 .

\begin{tabular}{|c|c|c|c|c|c|c|c|c|c|c|c|}
\hline Day. & Aug. & Sept.| & Oct. & Nov. & Dec & Day. & Aug. & Sept. & Oct. & Nov. & Dec. \\
\hline & & 10 & .95 & .00 & 1.10 & & 1.80 & 3.90 & 1.10 & 1.05 & 1.45 \\
\hline & & & .9 & & 1.15 & & & & 1.00 & & 1.30 \\
\hline & 1.10 & 1. & .90 & 2.00 & 1.25 & & 1.10 & 1.10 & 1.05 & & 2.40 \\
\hline & 1.10 & 1.3 & .90 & 1.80 & 1.40 & & 1.00 & 1.05 & 1.05 & & 4.50 \\
\hline & 1.10 & 1.2 & 3.50 & 1.40 & 1.35 & 21 & 1.00 & 1.00 & 1.00 & & 3.50 \\
\hline & 1.05 & 1.05 & 1.80 & 1.20 & 1.30 & 22 & .90 & 1.00 & 1.50 & & 1.70 \\
\hline & 1.05 & 1.00 & 1.2 & 1.20 & 1.25 & 23 & .90 & 1.00 & 1.45 & & 1.50 \\
\hline & 1.00 & 1.0 & 1. & 1.20 & 1.20 & 24 & 1.70 & 1.00 & 1. & & 1.40 \\
\hline & 1.05 & $\because$ & & 1.15 & 1.15 & & 1.40 & 1.00 & 1. & & 1.40 \\
\hline & 1.00 & 2. & & 1.15 & 1.10 & & 1.60 & 1.00 & 1.15 & & 1.35 \\
\hline & 1. & 1.8 & 1.1 & 1.15 & 1.10 & & 1.15 & 1.05 & 1.10 & & 1.35 \\
\hline & 9.0 & 1.4 & & 1.1 & 1.10 & & 1.10 & 1.05 & 1. & & 1.35 \\
\hline & 9. & 1.2 & 1.3 & 1.1 & 1.10 & 29 & 1.00 & 1.00 & 1.05 & & 1.30 \\
\hline & 9.00 & 1.20 & 1.1 & 1.10 & 2.20 & 30 . & 1.00 & .90 & 1.00 & 1.10 & 1.75 \\
\hline & 1.80 & 2.0 & 1.1 & 1.10 & 1.80 & & 1.80 & & 1.05 & & 2.00 \\
\hline & 1.00 & 2.20 & 1.05 & 1.10 & 1.45 & & & & & & \\
\hline
\end{tabular}


Daily gage height, in feet, of Big Sandy Creek near Dadeville, Ala., for 1901 .

\begin{tabular}{|c|c|c|c|c|c|c|c|c|c|c|c|c|}
\hline Day. & Jan. & Feb. & Mar. & April & May & June & July & Aug. & Sept. & Oct. & Nov. & Dec \\
\hline & & & & & .40 & 1.40 & & . 10 & 1.30 & 4.20 & .85 & \\
\hline & & & & & & & & & & & .85 & \\
\hline & & & & & 1. & & & & & & .85 & 1.40 \\
\hline & & & & & & & & & & & .85 & 1. \\
\hline & 1.6 & & & & 1.35 & & & & 1. & & .85 & 1. \\
\hline & & & & & 1.3 & 3. & & & & & .90 & 1.3 \\
\hline & 1.4 & & 1.8 & & 1.40 & 2. & & & 1. & & .90 & 1.1 \\
\hline & 1.4 & & & & & & & & & & 1.00 & 1. \\
\hline & 1.4 & & 1.4 & & 1.35 & 1.5 & 1.2 & & 1.00 & & 1.00 & 1.1 \\
\hline & 1.4 & & 1.4 & & 1.3 & 1. & & & .90 & & 1.00 & 1. \\
\hline & 1.9 & & & & 1.30 & 1. & 1. & & .9 & & 1.00 & 1.0 \\
\hline & 1.7 & & 1. & & 1. & 2. & & & .8 & & .90 & 1. \\
\hline & 2.5 & & 1.5 & & 1.3 & 1.8 & & & .8 & & 1.00 & 1.1 \\
\hline & 1.9 & & & & 1.5 & 1. & & & 1.4 & & 1.00 & 4.4 \\
\hline & 1.6 & & & & 1.50 & & 1. & & 1.4 & & 1.00 & 3.8 \\
\hline & 1.5 & 1.4 & 1.3 & & 1.4 & 1. & 1. & & & & 1.00 & 3.0 \\
\hline & & & & & 1.2 & 1.5 & & & & & 1.00 & 2.9 \\
\hline & 2.0 & & 1.3 & & 1.4 & 1. & & & & & .90 & 2.5 \\
\hline & 1.6 & & & & & & & & & & .90 & 2.4 \\
\hline & 1.5 & & & & 1.7 & & & & & & .80 & 2.4 \\
\hline & & & & & & & & & & & .80 & 2.0 \\
\hline & 1.4 & 1.4 & & & 3. & 1. & & & & & 1.00 & 2.0 \\
\hline & & & & & & & & & & & 1.00 & 1.9 \\
\hline & 1.4 & 1.5 & & & 1. & 1. & & & & 1. & .85 & 1.8 \\
\hline & 1.5 & & & & & & & & & & .85 & 1.5 \\
\hline & 1.5 & 1.4 & 1. & & & 1. & 1. & & & & .80 & 3.0 \\
\hline & 1.4 & 1.4 & & & & 1. & & & & & .90 & 3.00 \\
\hline & 1.4 & 1.4 & & & & 1. & 1. & & & & .90 & 21.00 \\
\hline & 1.4 & & & 1.4 & 1.5 & 1. & & & & & .90 & 16.00 \\
\hline & 1.4 & & 2.30 & 1.45 & 1.4 & 1.20 & & & 1.80 & $* .70$ & .90 & 8.00 \\
\hline & 1.40 & $\cdots \cdots$ & |13.10 & & 1.40 & & 1.10 & 1.40 & & .80 & $\cdots$ & 4.00 \\
\hline
\end{tabular}

*Water was being held back by dams above in the morning when readings were made; 0.8 is assumed as minimum for October. 
Rating table for Big Sandy Creek at Dadeville, Ala., for 1900 and 1901.

\begin{tabular}{|c|c|c|c|c|c|c|c|}
\hline 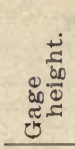 & 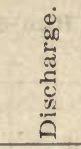 & 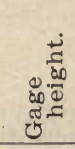 & 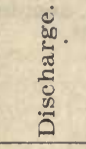 & 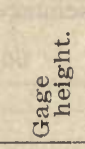 & 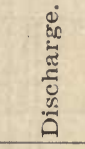 & 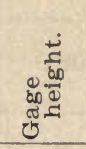 & 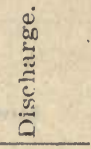 \\
\hline 0.8 & 67 & 4.4 & 1,868 & 8.0 & 3,740 & 11.6 & 5,612 \\
\hline 0.9 & 85 & 4.5 & 1,920 & 8.1 & 3,792 & 11.7 & 5,664 \\
\hline 1.0 & 115 & 4.6 & 1,972 & 8.2 & 3,844 & 11.8 & 5,716 \\
\hline 1.1 & 152 & 4.7 & 2,024 & 8.3 & 3,896 & 11.9 & 5,768 \\
\hline 1.2 & 204 & 4.8 & 2,076 & 8.4 & 3,948 & 12.0 & 5,820 \\
\hline 1.3 & 256 & 4.9 & 2,128 & 8.5 & 4,000 & 12.1 & 5,872 \\
\hline 1.4 & 308 & 5.0 & 2,180 & 8.6 & 4,052 & 12.2 & 5,924 \\
\hline 1.5 & 360 & 5.1 & 2,232 & 8.7 & 4,104 & 12.3 & 5,976 \\
\hline 1.6 & 412 & 5.2 & 2,284 & 8.8 & 4,156 & 12.4 & 6,028 \\
\hline 1.7 & 464 & 5.3 & 2,336 & 8.9 & 4,208 & 12.5 & 6,080 \\
\hline 1.8 & 516 & 5.4 & 2,388 & 9.0 & 4,260 & 12.6 & 6,132 \\
\hline 1.9 & 568 & 5.5 & 2,440 & 9.1 & 4,312 & 12.7 & 6,184 \\
\hline 2.0 & 620 & 5.6 & 2,492 & 9.2 & 4,364 & 12.8 & 6,236 \\
\hline 2.1 & 672 & 5.7 & 2,544 & 9.3 & 4,416 & 12.9 & 6,288 \\
\hline 2.2 & 724 & 5.8 & 2,596 & 9.4 & 4,468 & 13.0 & 6,340 \\
\hline 2.3 & 776 & 5.9 & 2,648 & 9.5 & 4,520 & 13.1 & 6,392 \\
\hline 2.4 & 828 & 6.0 & 2,700 & 9.6 & 4,572 & 13.2 & 6,444 \\
\hline 2.5 & 880 & 6.1 & 2,752 & 9.7 & 4,624 & 13.3 & 6,496 \\
\hline 2.6 & 932 & 6.2 & 2,804 & 9.8 & 4,676 & 13.4 & 6,548 \\
\hline 2.7 & 984 & 6.3 & 2,856 & 9.9 & 4,728 & 13.5 & 6,600 \\
\hline 2.8 & 1,036 & 6.4 & 2,908 & 10.0 & 4,780 & 13.6 & 6,652 \\
\hline 2.9 & 1,088 & 6.5 & 2,960 & 10.1 & 4,832 & 13.7 & 6,704 \\
\hline 3.0 & 1,140 & 6.6 & 3,012 & 10.2 & 4,884 & 13.8 & 6,756 \\
\hline 3.1 & 1,192 & 6.7 & 3,064 & 10.3 & 4,936 & 13.9 & 6,808 \\
\hline 3.2 & 1,244 & 6.8 & 3,116 & 10.4 & 4,988 & 14.0 & 6,960 \\
\hline 3.3 & 1,296 & 6.9 & 3,168 & 10.5 & 5,040 & 14.1 & 6,912 \\
\hline 3.4 & 1,348 & 7.0 & 3,220 & 10.6 & 5,092 & 14.2 & 6,964 \\
\hline 3.5 & 1,400 & 7.1 & 3,272 & 10.7 & 5,144 & 14.3 & 7,016 \\
\hline 3.6 & 1,452 & 7.2 & 3,324 & 10.8 & 5,196 & 14.4 & 7,068 \\
\hline 3.7 & 1,504 & 7.3 & 3,376 & 10.9 & 5,248 & 14.5 & 7,120 \\
\hline 3.8 & 1,556 & 7.4 & 3,428 & 11.0 & 5,300 & 14.6 & 7,172 \\
\hline 3.9 & 1,608 & 7.5 & 3,480 & 11.1 & 5,352 & 14.7 & 7,224 \\
\hline 4.0 & 1,660 & 7.6 & 3,532 & 11.2 & 5,404 & 14.8 & 7,276 \\
\hline 4.1 & 1,712 & 7.7 & 3,584 & 11.3 & 5,456 & 14.9 & 7,328 \\
\hline 4.2 & 1,764 & 7.8 & 3,636 & 11.4 & 5,508 & 15.0 & 7,380 \\
\hline 4.3 & 1,816 & 7.9 & 3,688 & 11.5 & 5,560 & & \\
\hline
\end{tabular}

Note.-This table applied to the foregoing "Daily gage heignts" gives the cubic feet per second flowing in the river on each date for which the gage height is given. 
Estimated monthly discharge of Big Sandy Creek near Dadeville, Ala.

[Drainage area, 195 square miles.]

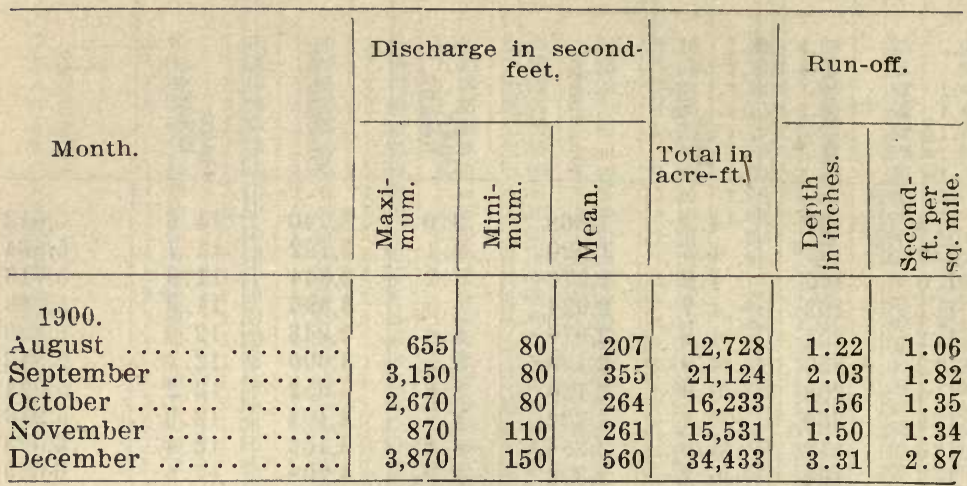

Estimated monthly discharge of Big Sandy Creek near Dadeville, Ala.

[Drainage area, 195 square miles.]

\begin{tabular}{|c|c|c|c|c|c|}
\hline \multirow[b]{2}{*}{ Month. } & \multicolumn{3}{|c|}{$\begin{array}{c}\text { Discharge in second- } \\
\text { feet. }\end{array}$} & \multicolumn{2}{|c|}{ Run-off. } \\
\hline & 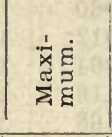 & 昙 & 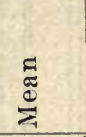 & 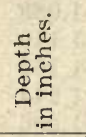 & 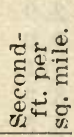 \\
\hline \multicolumn{6}{|l|}{1901.} \\
\hline January $\ldots \ldots \ldots \ldots \ldots \ldots \ldots$ & 880 & 308 & 425 & 2.51 & 2.18 \\
\hline 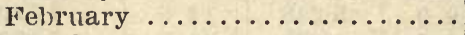 & 2,700 & 282 & 545 & 2.90 & 2.78 \\
\hline March $\ldots \ldots \ldots \ldots \ldots \ldots \ldots$ & 6,392 & 256 & 552 & 3.26 & 2.83 \\
\hline April $\ldots \ldots \ldots \ldots \ldots \ldots$ & 3,428 & 334 & 689 & 3.94 & 3.53 \\
\hline 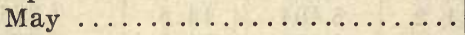 & 3,220 & 230 & 480 & 2.84 & 2.46 \\
\hline June $\ldots \ldots \ldots \ldots \ldots \ldots \ldots$ & 2,492 & 178 & 523 & 2.99 & 2.68 \\
\hline July $\ldots \ldots \ldots \ldots \ldots \ldots \ldots$ & 516 & 152 & 227 & 1.34 & 1.16 \\
\hline August $\ldots \ldots \ldots \ldots \ldots \ldots \ldots$ & 2,180 & 115 & 369 & 2.18 & 1.89 \\
\hline September $\ldots \ldots \ldots \ldots \ldots \ldots$ & 620 & 67 & 257 & 1.47 & 1.32 \\
\hline October $\ldots \ldots \ldots \ldots \ldots \ldots$. & 1,764 & $* 45$ & 462 & 2.73 & 2.37 \\
\hline November $\ldots \ldots \ldots \ldots \ldots \ldots$ & 115 & 67 & 92 & .52 & .47 \\
\hline December $\ldots \ldots \ldots \ldots \ldots \ldots$ & 10,500 & 85 & 1,265 & 7.48 & 6.49 \\
\hline The year $\ldots \ldots \ldots \ldots$ & 10,500 & *45 & 490 & 34.16 & 2.51 \\
\hline
\end{tabular}

*See foot note under gage heights for 1901 . 
Minimum monthly discharge of Big Sandy Creek at Dadeville, Ala., with corresponding net horsepower per foot of fall on a water wheel realizing 80 per cent of the theoretical power.

[Drainage area, 195 square miles.]

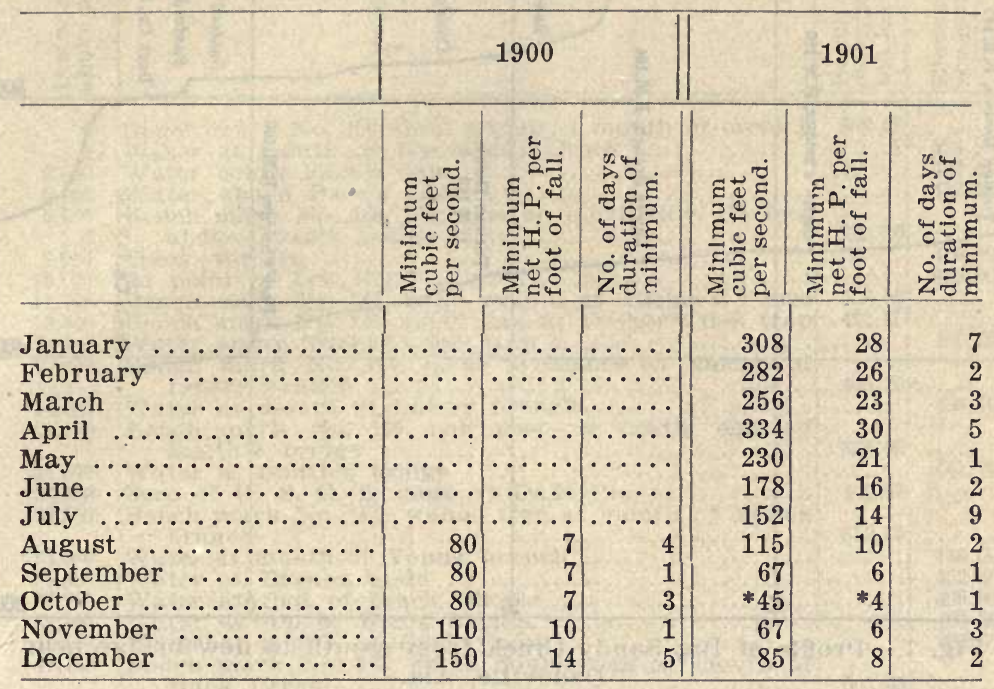

*Note. - To find the minimum net horse power available at a shoal on this stream, near this station, for any month, multiply the total fall of the shoal by the "Net H. P. per foot of fall" in this table for that month.

*See foot note under gage heights for 1901.

A survey made in July, 1900, of Big Sandy Creek from its mouth to the new bridge near Dadeville, Ala., showed a total fall of 157 feet in a distance of 65,000 feet, or about 12 miles. 


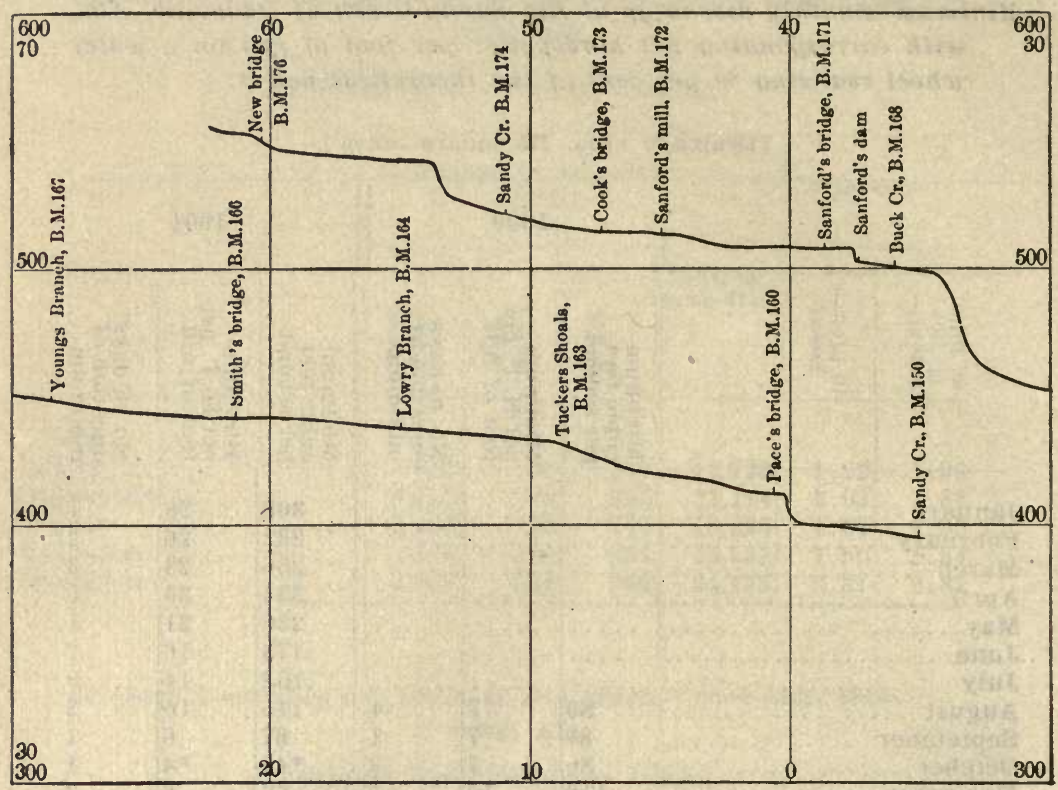

Fig. \%.-Profile of Big Sandy Creek from mouth to new bridge near Dadeville, Ala.

*Note.-The numbers $0,10 \ldots 70$, represent thousand feet stations.

The profile resulting from the survey is reproduced in Fig. 7. The following list of distances and elevations of water and bench marks shows the fall of the creek from point to point. 
Elevations and bench marks along Big sandy Creek between its mouth and the new bridge near Dadeville, Ala.

\begin{tabular}{|c|c|c|c|}
\hline 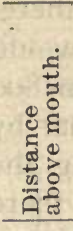 & Description or location. & 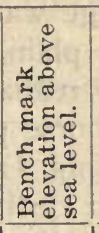 & 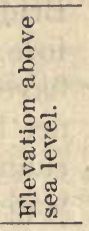 \\
\hline & ench mark No. 150 , dead stump at mouth of cre & 398.08 & \\
\hline & ater at mouth of Big Sandy Creek............. & ......... & \\
\hline $\begin{array}{l}5,000 \\
5,000\end{array}$ & ater below Pace's dam ............................ & (......... & 402 \\
\hline $\begin{array}{l}5,0 \\
5,6\end{array}$ & $\begin{array}{l}\text { Water above Pace's dam } \ldots \ldots \ldots \ldots \ldots \ldots \ldots \ldots \ldots \ldots \ldots \ldots \ldots \\
\text { Bench mark No. } 160 \text {, big pine on north side, } 175 \text { feet }\end{array}$ & & \\
\hline & 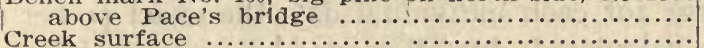 & 422.30 & 416. \\
\hline & $\begin{array}{l}\text { Creek surface } \ldots \ldots \ldots \ldots \ldots \ldots \ldots \ldots \ldots \ldots \ldots \ldots \\
\text { At point of Ivy Bend } \ldots \ldots \ldots \ldots \ldots \ldots \ldots\end{array}$ & & $\begin{array}{l}416.00 \\
419.00\end{array}$ \\
\hline & Bench mark No. 162 , large walnut at Tu & $\ddot{503.85}$ & \\
\hline & Bench mark No. 163 , small oak at Tucker's fish t & 432.85 & \\
\hline & Vater above Tucker's fish trap $\ldots \ldots \ldots \ldots \ldots \ldots \ldots \ldots$ & & 430.0 \\
\hline & $\begin{array}{c}\text { Bench mark No. } 164 \text {, large sycamore at mouth of } \\
\text { Lowry branch } \ldots \ldots \ldots \ldots \ldots \ldots \ldots \ldots \ldots \ldots \ldots \ldots \ldots \ldots \ldots \ldots \ldots\end{array}$ & 445.20 & \\
\hline & 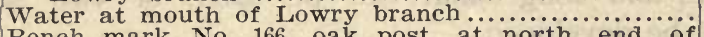 & & 436.1( \\
\hline & $\begin{array}{c}\text { Bench mark No. } 166, \text { oak post at north end of } \\
\text { Smith's bridge } \ldots \ldots \ldots \ldots \ldots \ldots \ldots \ldots \ldots \ldots \ldots \ldots \ldots \ldots \ldots \ldots \ldots \ldots \ldots\end{array}$ & 463.95 & \\
\hline & Water at Smith's bridge $\ldots \ldots \ldots \ldots \ldots \ldots \ldots \ldots \ldots \ldots$ & & 441. \\
\hline & $\begin{array}{l}\text { Zero of U. S. G. S. gage at Dadeville } \ldots \ldots \ldots \ldots \ldots \\
\text { Bench mark No. } 167 \text {, wahoo tree at mouth of Young }\end{array}$ & 440.50 & \\
\hline & & 559.58 & \\
\hline & at mouth of Young branch $\ldots \ldots \ldots \ldots \ldots \ldots \ldots . . .$. & $\cdots$ & \\
\hline & er at Barnes basin ... & & \\
\hline & ter at foot of Black Shoals..... & & \\
\hline & ter at top of Black Shoals................... & & 496. \\
\hline & Water at mouth of Buck creek......................... & & \\
\hline & & 503.65 & \\
\hline & $\begin{array}{l}\text { dy water below San } \\
\text { nch mark No. } 169 \text {, hi }\end{array}$ & $\ddot{5} \ddot{2} \ddot{2} . \dot{10}$ & 500.1 \\
\hline & 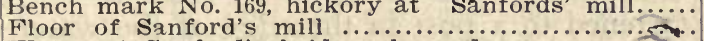 & $\begin{array}{l}522.10 \\
514.00\end{array}$ & \\
\hline & ter at Sanford's b & & \\
\hline & ord Pond $\ldots \ldots \ldots \ldots$ & & \\
\hline & Water at second shoal above Sanford Pond $\ldots . . . . .$. & & 512.5 \\
\hline & $\begin{array}{l}\text { Bench mark No. } 173 \text {, large white oak near north end } \\
\text { of Cook's bridge } \ldots \ldots \ldots \ldots \ldots \ldots \ldots \ldots \ldots \ldots \ldots\end{array}$ & 539.35 & \\
\hline & $r$ at Cook's i & & \\
\hline & Water opposite mouth of Chattasofka Creek.......... & & 520.6 \\
\hline & $\begin{array}{l}\text { Bench mark, 16-inch water oak on west bank of } \\
\text { Chattasofka Creek, } 50 \text { feet above mouth........... }\end{array}$ & & \\
\hline & actory shoal ....... & & \\
\hline & 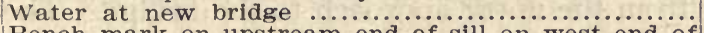 & & \\
\hline & $\begin{array}{l}\text { Bench mark on upstream end of sill on west end of } \\
\text { new bridge } \ldots \ldots \ldots \ldots \ldots \ldots \ldots \ldots \ldots \ldots \ldots \ldots \ldots \ldots \ldots \ldots \ldots \ldots \ldots \ldots\end{array}$ & & \\
\hline & neh mar & & \\
\hline
\end{tabular}

Water supply o1 this creek is shown by the foregoing records of Dadeville Hydrographic Station at Smith's Bridge.

The best shoal on this creek is the Sanford and Black Shoal, near Dadeville, which has a fall of 85.8 feet, in a distance of 5.2 miles. With a dam 54 feet high, and a canal I, 370 feet long, a practical working head of 80 feet can be developed, having I foot extra for grade of canal, and 4 feet extra for storage at top of dam. The foregoing record shows that from 
August I, I900, to December 3I, I90I, covering a record of 515 days, there were only 37 days in which the flow at Smith's Bridge was less than II 5 cubic feet per second. It is, therefore, plain that during the last two years such a plant would have realized, for 90 per cent. of the time, not less than 800 net horse power continuously, 24 hours per day; and that by running I I hours per day, 6 days per week, and storing the water during the time that the wheels are standing, there would have been $2,000 \mathrm{H}$. P. or more for use during factory hours, for 90 per cent. of the time during the last two years. By applying the rating table to the gage heights, and finding the discharge for each individual day, the exact power obtainable can be calculated, due allowance being made for the storage capacity, and equalizing effect of the dam.

Of course, this 85 foot fall can be developed in other ways. A low dam, and long canal can be used, or two separate powers can be developed.

\section{HILlABEE CREEK, NEAR ALEXANDER CITY, ALABAMA.}

This station, which was established August 20, I900, by J. $\mathrm{R}$. Hall, is located $61 / 2$ miles northeast off Alexander City, on the road leading from that town to Newsite. The gage, which is graduated to feet and tenths, and is placed vertically, is in two sections, the short sẹction, which reads from o to 5.50 feet, being fastened to a post in the edge of the water on the north bank 20 feet from the upstream side of the bridge, the long section, which reads from 5.50 feet to 16 feet, being fastened to the upstream end of the first pier on the north bank, and arranged so that when water rises above the short section the readings are made from the long one, both sections being easily read from the north approach to the bridge. The initial point of sounding is on the south side of the first pier on the north bank. The gage is referred to a bench mark at the top of a chord on the dawnstream side of the bridge at the second pier from the north bank, and is 27.6 feet above the zero of the gage. The bridge is in three spans, having a total length of 276 feet, with a north approach of I 6 feet and a south approach of 124 feet, making a total, over all, of 516 feet. The observer is J. H. Chisholm, a farmer, postoffice address Alexander City, Ala. During 1900 the following measurements were made by James R. Hall:

August 29: Gage height, 1.40 feet; discharge 184 second-feet. November 28: Gage height, 2 feet; discharge, 390 second-feet. 
Daily gage height of Hillabee Creek at Alexander City, Ala., for 1900.

\begin{tabular}{|c|c|c|c|c|c|c|c|c|c|c|c|}
\hline Day. & Aug. & Sept. & Oct. & Nov. & Dec & Day. & Aug. & Sept. & Oct. & Nov. & Dec \\
\hline 1 & & 2.30 & 1.30 & 1.60 & 1.90 & 17 & & 2.60 & 1.30 & 1.50 & 2.70 \\
\hline & & 2.3 & 1.1 & 2.60 & 1.90 & & & 2.2 & 1.3 & & 2.80 \\
\hline & & 1.60 & 1.10 & 6.80 & 1.80 & 19. & & 1. & & & 3.00 \\
\hline & & 1.4 & 1.1 & 3.20 & 1.9 & 20. & & 1.5 & 1.2 & & 2.90 \\
\hline & & 1.3 & 3.2 & 2.20 & 1.8 & 21. & & 1.5 & 1.2 & & 6.00 \\
\hline & & 1.2 & 2.0 & 1.80 & 1.9 & 22 . & . & 1.4 & 1. & & 4.00 \\
\hline & & 1.20 & 2.8 & 1.7 & 1.8 & 23. & & 1. & & & 3.0 \\
\hline & & 1.1 & 2.6 & 1.7 & 1.7 & 24. & . & 1.3 & 2. & & 2. \\
\hline & & 1.1 & 2.4 & 1.8 & $1 . ?$ & 25. & & 1. & & & 2.90 \\
\hline & & 1.2 & 2.3 & 1.7 & 1. & 26. & & 1.3 & 1. & & 2.8 \\
\hline & & 1.1 & 2.3 & 1.70 & 1.7 & 27. & & 1.40 & 1.8 & & 2.60 \\
\hline & & 1.1 & 1.8 & 1.7 & $1 . ?$ & 28. & & 1.3 & 1. & & 2. \\
\hline & & 1.1 & 1.60 & 1.70 & 1.80 & 29. & 1.40 & 1.40 & 1.6 & & 2.50 \\
\hline & & & 1.4 & 1.70 & 3.8 & 30. & 1.30 & 1.30 & & 1.90 & \\
\hline & & 8.1 & 1.4 & 1.70 & 2.9 & & 1.80 & & 1.50 & & \\
\hline & & 5.00 & 1.20 & 1.60 & & & & & & & \\
\hline
\end{tabular}

Daily gage height of Hillabee Creek at Alexander City, Ala., for 1901.

\begin{tabular}{|c|c|c|c|c|c|c|c|c|c|c|c|c|}
\hline Day & Jan. & Feb. & Mar. & April & May & Jun. & July & Aug. & s'ept. & Oct. & Nov. & Dec \\
\hline & 5.00 & 2.40 & 2.30 & 5.20 & 2.50 & 2.50 & 2.50 & 1.80 & 2.60 & 1.20 & 1.00 & 1.00 \\
\hline & & 2.40 & 2.30 & 4.40 & 2.50 & 2.40 & 2.50 & 1.40 & 2.00 & 1.20 & 1.00 & 1.00 \\
\hline & 4.90 & 4.60 & 2.40 & 4.80 & 2.50 & 3.00 & 2.40 & 1.40 & 1.40 & 1.10 & 1.00 & 2.00 \\
\hline & 4.7 & 9.40 & 2.40 & 3.10 & 2.40 & 2.50 & 2.30 & 1.20 & 1.20 & 1.10 & 1.00 & 1.60 \\
\hline & 3.9 & 6.00 & 2.30 & 2.90 & 2.40 & 2.50 & 2.00 & 1.10 & 1.10 & 1.10 & 1.30 & 1.40 \\
\hline & 3.60 & 4.10 & 2.20 & 2.80 & 2.40 & 2.60 & 1.80 & 1.10 & 1.10 & 1.20 & 1.10 & 1. 0 \\
\hline & 3.6 & 3.60 & 2.10 & 2.70 & 2.40 & 2.50 & 2.90 & 1.10 & 1.10 & 1.40 & 1.10 & 1.20 \\
\hline & 3.40 & 3.10 & 2.10 & 2.60 & 2.40 & 2.50 & 2.10 & 1.00 & 1,10 & 1.20 & 1.00 & 1.20 \\
\hline & 2.60 & 3.6 & 2.10 & 2.60 & 2.30 & 2.40 & 1.80 & 1.20 & N10 & 1.20 & 1.00 & 1.20 \\
\hline & 2.50 & 3.00 & 2.40 & 2.50 & 2.30 & 2.40 & 1.80 & 1.20 & 1.10 & 1.20 & 1.00 & 1.90 \\
\hline 11. & 8.00 & 2.90 & 2.50 & 2.50 & 2.30 & 2.30 & 1.70 & 1.10 & 1.00 & 1.60 & 1.00 & 1.50 \\
\hline & 7.60 & 2.90 & 2.30 & 2.40 & 2.20 & 2.00 & 1.60 & 1.10 & 1.00 & 2. & 1.00 & 1.40 \\
\hline 13. & 7.00 & 2.80 & 2.20 & 3.10 & 2.50 & 2.10 & 1.50 & 1.20 & 1.20 & 2.90 & 1.00 & 1.40 \\
\hline & 5.90 & 3.00 & 2.20 & 2.90 & 2.30 & 2.00 & 1.40 & 1.60 & 2.50 & 1.60 & 1.00 & 2.0 \\
\hline & 4.50 & 3.10 & 2.30 & 2.80 & 2.50 & 2.00 & 1.30 & 2.10 & 2.40 & 1.40 & 1.00 & 3.00 \\
\hline & 4.30 & 2.60 & 2.40 & 2.70 & 2.20 & 1.90 & 1.40 & 4.40 & 2.40 & 1.20 & 1.00 & 3.00 \\
\hline & 4.00 & 2.60 & 2.20 & 2.60 & 2.20 & 2.20 & 1.3 & 2.90 & 2.00 & 1.00 & 1.00 & 2.80 \\
\hline & 3.50 & 2.40 & 2.20 & 2.60 & 2.10 & 1.90 & 3.40 & 2.00 & 1.80 & 1.0 & 1.00 & $2 . \therefore 0$ \\
\hline & & 2.60 & 2.10 & 10.00 & 2.1 & 1.80 & 2.2 & 2.00 & 1. & 1. & 1.30 & 260 \\
\hline & 3.00 & 2.70 & 2.10 & 3.20 & 2.90 & 1.80 & 1.80 & 4.00 & 1.40 & i. & 1.30 & 2.00 \\
\hline & 2.90 & 2.60 & 3.0 & 3.1 & 3.80 & 1.70 & 2.00 & 4.10 & 1.20 & 1.0 & 1.30 & 1.50 \\
\hline & 2.90 & 2.40 & 2.40 & 3.00 & 2.90 & 1.70 & 1.90 & 3.40 & 1.40 & 1.0 & 1.30 & 1.50 \\
\hline & & 2.60 & 2.3 & 2.90 & 2.50 & 1.60 & 1.80 & 3.10 & 1.20 & 1.0 & 1.30 & 1.50 \\
\hline & 2.60 & 2.60 & 2.80 & 2.80 & 2.50 & $1.6 \mathrm{f}$ & 1.80 & 2. & 1.10 & 1. & 1.3 & 1.60 \\
\hline & & 2.50 & 2.70 & 2.70 & 2.40 & $1.7 \mathrm{f}$ & 1.70 & 2.20 & 1.10 & 1.0 & 1.20 & 1.60 \\
\hline & 2.60 & 2.40 & 3.50 & 2.60 & 3.90 & 1.70 & 1.70 & 2.2 & 1.20 & 1. & 1.20 & 3.70 \\
\hline & 2.60 & 2.40 & 3.00 & 2.50 & 2.80 & 1.80 & 1.40 & 2.10 & 2.10 & 1. & 1.10 & 1.70 \\
\hline & 2.50 & 2.40 & 2.70 & 2.50 & 2.6 & 1.80 & 1.60 & 3.8 & 1.60 & $1 .($ & 1.10 & 1.80 \\
\hline & 2.40 & -6 & 2.60 & 2.50 & 2.5 & 1.70 & 1.40 & 3.60 & 1.40 & 1. & 1.10 & $11 . \mathrm{r}^{-1}$ \\
\hline & 2.40 & ....... & 2.70 & 2.50 & 2.30 & 2.60 & 1.40 & 3.40 & 1.20 & 1. & 1.10 & \\
\hline & 2.40 & ....... & 5.20 & 年 & 2.90 & & 2.00 & 3.00 & & 1.00 & & 3.90 \\
\hline
\end{tabular}


Rating table for Hillabee Creek at Alexander City, Ala., for years 1900 and 1901.

\begin{tabular}{|c|c|c|c|c|c|}
\hline 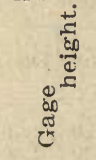 & 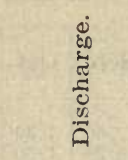 & 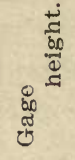 & 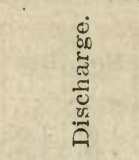 & & 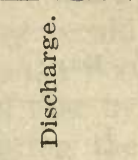 \\
\hline Feet. & Second $F^{\prime} t$. & Feet. & Second Feet. & Fret. & Second Ft. \\
\hline 1.0 & 138 & 2.4 & 566 & 3.8 & 1,182 \\
\hline 1.1 & 146 & 2.5 & 610 & 3.9 & 1,226 \\
\hline 1.2 & 156 & 2.6 & 564 & 4.0 & 1,270 \\
\hline 1.3 & 169 & 2.7 & 698 & 4.1 & 1,314 \\
\hline 1.4 & 184 & 2.8 & 742 & 4.2 & 1,358 \\
\hline 1.5 & 204 & 2.9 & 786 & 4.3 & 1,402 \\
\hline 1.6 & 230 & 3.0 & 830 & 4.4 & 1,446 \\
\hline 1.7 & 263 & 3.1 & 874 & 4.5 & 1,490 \\
\hline 1.8 & 303 & 3.2 & 918 & 4.6 & 1,534 \\
\hline 1.9 & 346 & 3.3 & 962 & 4.7 & 1,578 \\
\hline 2.0 & 390 & 3.4 & 1,006 & 4.8 & 1,622 \\
\hline 2.1 & 434 & 3.5 & 1,050 & 4.9 & 1,666 \\
\hline 2.2 & 478 & 3.6 & 1,094 & 5.0 & 1,710 \\
\hline 2.3 & 522 & 3.7 & 1,138 & & \\
\hline
\end{tabular}

NotE.-This table applied to the foregoing "Daily Gage Heights" gives the cubic feet per second flowing in the river on each date for which the gage height is given. 
Estimated monthly discharge of Hillabee Creek near Alexander City, Ala.

[Drainage area, 214 square miles.]

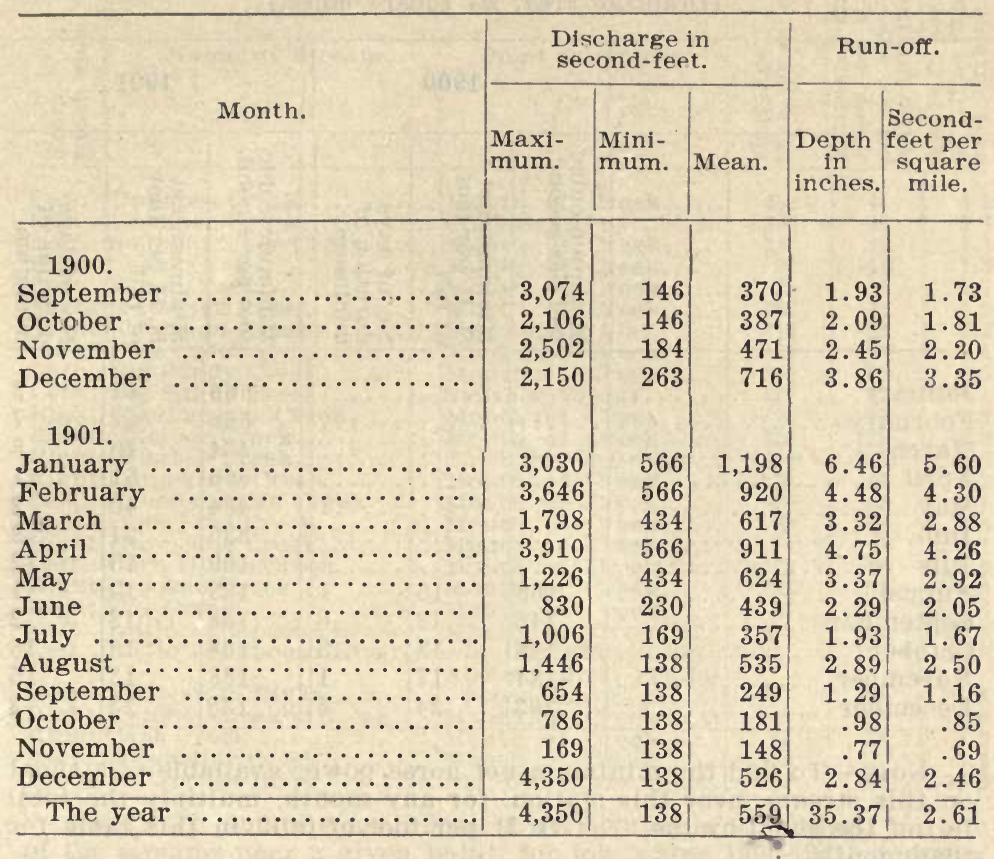


Minimum monthly discharge of Hillabee Creek at Alexander City, Ala., with corresponding net horsepower per foot of fall on a water wheel realizing 80 per cent of the theoretical power.

[Drainage area, 214 square miles.]

\begin{tabular}{|c|c|c|c|c|c|c|}
\hline & \multicolumn{3}{|c|}{1900} & \multicolumn{3}{|c|}{1901} \\
\hline & 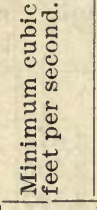 & 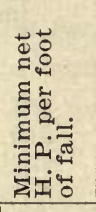 & 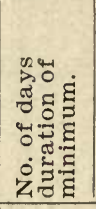 & 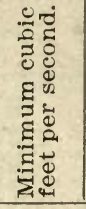 & 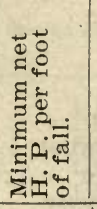 & 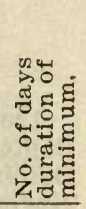 \\
\hline January & & & & 566 & 51 & 3 \\
\hline February $\quad \ldots \ldots \ldots \ldots \ldots$ & . & $\cdots$ & & 566 & 51 & 7 \\
\hline March ................ & & & & 434 & 40 & $\mathbf{5}$ \\
\hline April $\ldots \ldots \ldots \ldots \ldots \ldots$ & & ... & & 566 & 51 & 1 \\
\hline May $\ldots \ldots \ldots \ldots \ldots \ldots$ & $\ldots$ & $\ldots \ldots$ & & 434 & 40 & 2 \\
\hline June $\ldots \ldots \ldots \ldots \ldots \ldots$ & & $\ldots$ & & 230 & 21 & 2 \\
\hline July $\ldots \ldots \ldots \ldots \ldots \ldots$ & & $\ldots$ & & 169 & 15 & 2 \\
\hline August $\ldots \ldots \ldots \ldots \ldots$ & 169 & 15 & 1 & 138 & 13 & 1 \\
\hline September $\ldots \ldots \ldots \ldots \ldots$ & 146 & 13 & 6 & 138 & 13 & 2 \\
\hline October $\ldots \ldots \ldots \ldots \ldots$ & 146 & 13 & 3 & 138 & 13 & 15 \\
\hline November $\ldots \ldots \ldots \ldots \ldots$ & 184 & 17 & 1 & 138 & 13 & 15 \\
\hline December $\ldots \ldots \ldots \ldots \ldots$ & 263 & 24 & 5 & 138 & 13 & 2 \\
\hline
\end{tabular}

Note.-To find the minimum net horse power available at a shoal on this stream, near this station, for any month, multiply the total fall of the shoal by the "Net H. P. per foot of fall" in this table for that month. 
7. ALABAMA TRIBUTARIES OF THE TALLAPOOSA RIVER, FROM MILSTEAD UP.

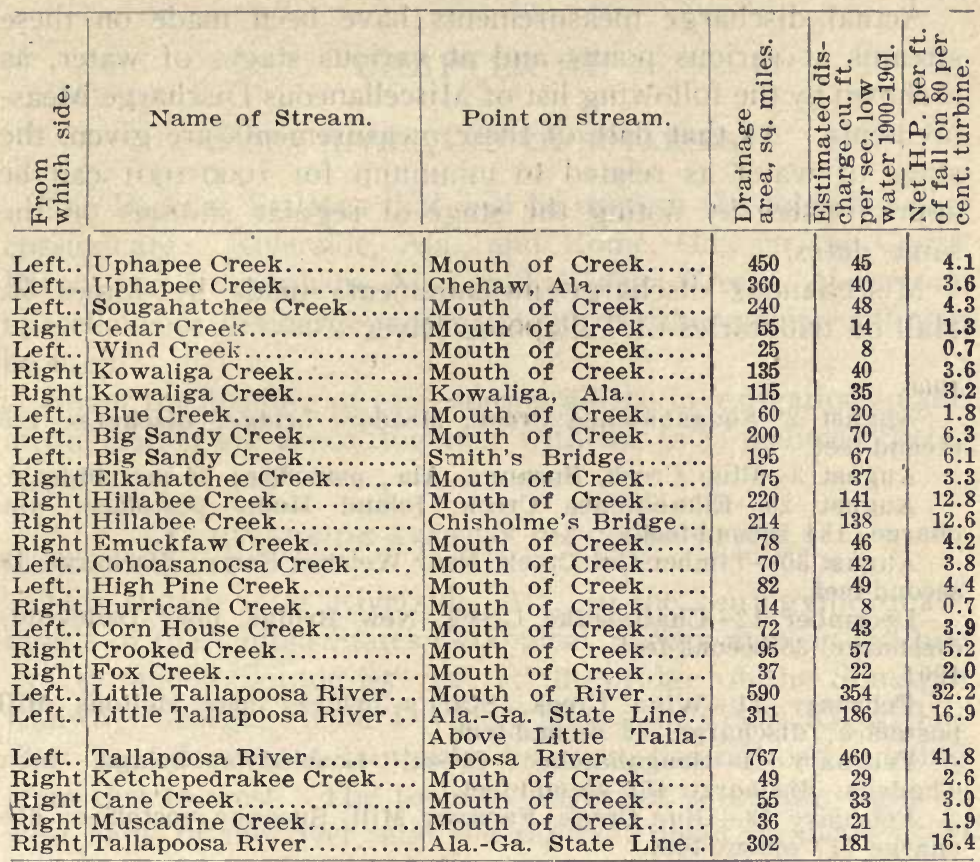

NoTE.-To find the net horse power available at shoal on one of the streams near a given point, for low water 1900-1901, multiply the total fall of the shoal by the "Net H. P. per foot of fall' in this table.

All of these tributaries to the Tallapoosa River are in the Crystalline region, and are very precipitous streams, having fine shoals all along their courses.

No State or Government Surveys have ever been made to determine their profiles, and it is, therefore, impossible at present to make a detailed statement of the water powers. The tabulated statement given above shows the cubic feet per second flowing in the streams, at certain places during low season of ordinary years, like 1900 and I90I.

This flow at any point multiplied by the total practical fall in feet that can be brought upon a water wheel on the given stream at that point, and divided by I I gives the net available horse power at that point, during low season of a year like 1900 or 1901 .

The "Cubic feet per second" flowing at the given points and the corresponding "Drainage areas" can be used to get by pro- 
portion the discharge at other points of same stream whose drainage areas are known.

Actual discharge measurements have been made on these streams at various points and at various stages of water, as is shown by the following list of Miscellaneous Discharge Measurements. As that date of these measurements are given, the stage of water as related to minimum for I9Oo-IgO I can be approximated by noting the stage at regular stations on the same dates.

Miscellanous discharge measurements made by James R. Hall on tributaries of Tallapoosa River.

1900.

August 2-Sougahatchee Creek, Meaders bridge; discharge 125 second-feet.

August 3-Blue Creek, Susanna, Ala., postoffice; 34 second-feet.

August 28-Elkahatchee Creek, Island Home postoffice; discharge, 184 second-feet.

August 30-Timber Cut Creek, Near Welches Ferry; discharge, 18 second-feet.

December 12 - Chattasofka Creek, New Bridge, near Dadeville; discharge, 35 second-feet. 1901.

February 11-Wind Creek, Starr's bridge, near Meltons Mill postoffice; discharge, 66 second-feet.

February 11-Sougahatchee Creek, Lovelady's bridge, near Thadeus; discharge 453 second-feet.

February 13-Blue Creek, Farrows Mill, Susanna postoffice; dis. charge 117 second-feet.

February 13-Channahatchee Creek, Freeman's Mill, Channahatchee postoffice; discharge 80 second-feet.

February 27-Kowaliga Creek, Benson's bridge, Kowaliga postoffice; discharge 154 second-feet.

March 5-Emuckfaw Creek, Hamlett's Mill, Zana postoffice; discharge 113 second-feet.

March 11-Moore's Creek, near Dudleyville; discharge 29 secondfeet.

March 12-Chattahaspa Creek, Scott's Mill, near Tiller Crossroads postoffice; discharge 203 second-feet.

March 12-Cohoasanocsa Creek, Leverett's Mill, near Milltown postoffice; discharge 122 second-feet.

March 12-High Pine Creek, Lile's Gin, Happy Land postoffice; discharge 89 second-feet.

March 12-Beaver Dam Creek, near Louina postoffice; discharge 30 second-feet.

March 13-Corn House Creek, Swann's Store, near Level Road postoffice; discharge 31 second-feet.

March 13-Wild Cat Creek, Murphy's Mill, near Gay postoffice; discharge, 32 second-feet.

March 13-Tallapoosa River, below mouth of Little Tallapoosa River, near Goldburg; discharge, 2,400 second-feet.

March 13-Crooked Creek, near Goldberg; discharge 183 second-feet.

March 13-Hurricane Creek, near Almond postoffice; discharge, 29 second feet. 


\section{CHAPTER III.}

\section{COOSA RIVER AND TRIBUTARIES.}

The Regular Stations that will be used in the folowing discussion are: Riverside, Ala., and Rome, Ga., on the Coosa River; and Nottingham, Ala., on Talladega Creek. Numerous miscellaneous discharge measurements at other points will also be used.

Under the heading of each station all the investigations made at the station are given, together with the facts deduced therefrom.

\section{RIVERSIDE STATION ON COOSA RIVER.}

This station is at Riverside, Ala., in the Springville quadrangle of the United States Geological Survey map, in latitude $33^{\circ} 37^{\prime}$ and longitude $85^{\circ} 12^{\prime}$, at the bridge of the Southern Railway, Georgia Pacific Division, across the Coosa River. The river here flows in a southerly direction, the railroad running from east to west. The town of Riverside is on the right or west bank of the river, and the railroad depot is about 1,000 feet west of the bridge, which is of iron and about 30 feet above low water. Beginning at the left bank, there are two spans of 154 feet each; then a drawbridge 220 feet, revolving on a large center pier; then a stationery span, 80 feet in length, to west or right bank abutment. There is no running water at low stages under the last-named span.

At low water the flowing river is 480 feet wide, including three piers, and is from 4 to Io feet deep. Very little of the current is too slow to turn any meter. It is somewhat irregular, as there are shoals and some old cribs just above the bridge, but for all stages it is probably the best station that can be found on the river at a bridge and easy of access.

On September 8, I896, a discharge measurement was made by B. M. Hall, and two bench marks were established. On September 22, 1896, another discharge measurement was made, a wire gage was put in, and Mr. J. W. Foster, sawyer at a large sawmill about 300 feet distant, on right bank of river, below the bridge, was employed as observer. 
The initial point is top of left abutment at the edge toward the river, on the downstream side of the bridge, from which side soundings and meter measurements are made. The rod of wire gage is nailed to outside guard rail, downstream side, next to the last panel of stationary bridge before reaching the pier at end of draw span. The rod is 14 feet long and divided to feet and tenths. The bench mark is the top of capstone on the large circular center pier of turn span. It is 26.80 feet above datum of gage at downstream side of pier.

The drainage area is 6,850 square miles, and is mapped on atlas sheets Springville, Anniston, Gadsden, Fort Payne, Rome, Tallapoosa, Marietta, Cartersville, Suwanee, Ellijay, Dalton, Cleveland, Ringgold, and Stevenson of the United States Geological Survey.

The following discharge measurements were made during I 896 by B. M. Hall and others :

September 8: Gage height, 0.70 feet; discharge, 1,630 second-feet.

September 25: Gage height, 0.50 feet; discharge, 1,403 second-feet.

October 30: Gage height, 0.88 feet; discharge, 1,986 second-feet.

December 21: wage height, 1.57 feet; discharge, 3,272 second-feet.

Daily gage height in feet of Coosa river at Riverside, Ala., for 1896.

\begin{tabular}{|c|c|c|c|c|c|c|c|c|c|}
\hline Day. & Sept.| & Oct.1 & Nov. & Dec. & Day. & [Sept.| & Oct. & Nov. & Dec. \\
\hline 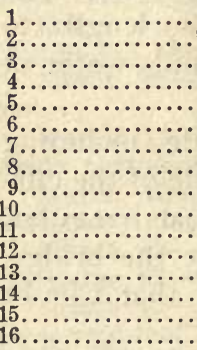 & & $\begin{array}{l}0.60 \\
1.75 \\
3.10 \\
2.75 \\
2.00 \\
1.50 \\
1.20 \\
.85 \\
.70 \\
.60 \\
.65 \\
.60 \\
.60 \\
.60 \\
.55 \\
.55\end{array}$ & $\begin{array}{l}1.10 \\
1.40 \\
1.20 \\
1.10 \\
1.05 \\
1.10 \\
1.20 \\
2.55 \\
2.30 \\
1.90 \\
1.30 \\
1.60 \\
2.25 \\
2.70 \\
4.00 \\
5.20\end{array}$ & $\begin{array}{l}1.30 \\
2.10 \\
4.38 \\
3.80 \\
3.20 \\
2.50 \\
2.20 \\
1.90 \\
1.70 \\
1.60 \\
1.55 \\
1.55 \\
1.60 \\
1.60 \\
1.80 \\
2.00\end{array}$ & 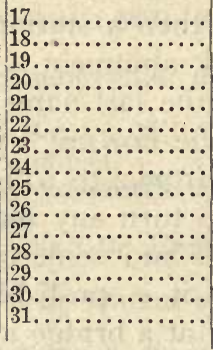 & 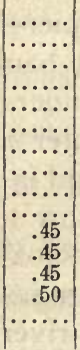 & $\begin{array}{r}0.55 \\
.65 \\
.80 \\
.85 \\
.75 \\
.70 \\
.60 \\
.55 \\
.60 \\
.70 \\
.80 \\
.85 \\
.90 \\
.95 \\
.85\end{array}$ & $\begin{array}{l}4.70 \\
4.20 \\
3.20 \\
2.30 \\
1.50 \\
1.40 \\
1.35 \\
1.30 \\
1.25 \\
1.20 \\
1.15 \\
1.15 \\
1.10 \\
1.20\end{array}$ & $\begin{array}{l}2.10 \\
2.20 \\
2.00 \\
1.80 \\
1.70 \\
1.50 \\
1.45 \\
1.40 \\
1.35 \\
1.30 \\
1.25 \\
1.20 \\
1.10 \\
1.10 \\
1.10\end{array}$ \\
\hline
\end{tabular}


Rating table for Coosa River at Riverside, Ala., for 1896.

\begin{tabular}{|c|c|c|c|c|c|}
\hline & 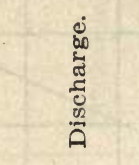 & 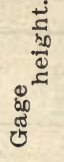 & 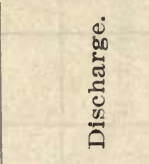 & 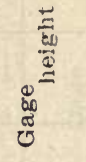 & 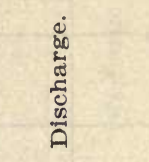 \\
\hline Feel. & Second $\boldsymbol{F}^{\prime} t$. & Feel. & Second Ft. & Feet. & Second Ft. \\
\hline 0.5 & 1,400 & 2.1 & 4,630 & 3.7 & 9,640 \\
\hline 0.6 & 1,500 & 2.2 & 4,920 & 3.8 & 9,980 \\
\hline 0.7 & 1,630 & 2.3 & 5,200 & 3.9 & 10,330 \\
\hline 0.8 & 1,780 & 2.4 & 5,500 & 4.0 & 10,680 \\
\hline 0.9 & 1,930 & 2.5 & 5,800 & 4.1 & 11,030 \\
\hline 1.0 & 2,100 & 2.6 & 6,100 & 4.2 & 11,390 \\
\hline 1.1 & 2,280 & 2.7 & 6,400 & 4.3 & 11,750 \\
\hline 1.2 & 2,480 & 2.8 & 6,700 & 4.4 & 12,110 \\
\hline 1.3 & 2,680 & 2.9 & 7,010 & 4.5 & 12,470 \\
\hline 1.4 & 2,880 & 3.0 & 7,320 & 4.6 & 12,840 \\
\hline 1.5 & 3,090 & 3.1 & 7,640 & 4.7 & 13,210 \\
\hline 1.6 & 3,320 & 3.2 & 7,970 & 4.8 & 13,580 \\
\hline 1.7 & 3,560 & 3.3 & 8,300 & 4.9 & 13,950 \\
\hline 1.8 & 3,820 & 3.4 & 8,630 & 5.0 & 14330 \\
\hline 1.9 & 4,080 & 3.5 & 8,960 & 5.1 & 14,710 \\
\hline 2.0 & 4,360 & 3.6 & 9,300 & 5.2 & 15,100 \\
\hline
\end{tabular}

Note.-This table applied to the foregoing "Daily gage heights" gives the cubic feet per second flowing in the river on each date for which the gage height is given.

The following discharge measurements were made during 1897 by Max Hall and others :

March 31: Gage height, 4.53 feet; discharge, 12,515 second-feet. June 17: Gage height, 1.54 feet; discharge, 3,747 second-feet.

July 21: Gage height, 5.55 feet; discharge, 16,925 second-feet.

August 20: Gage height, 2.58 feet; discharge, 6,174 second-feet.

November 29: Gage height, 0.80 feet; discharge, 1,854 second-feet. 


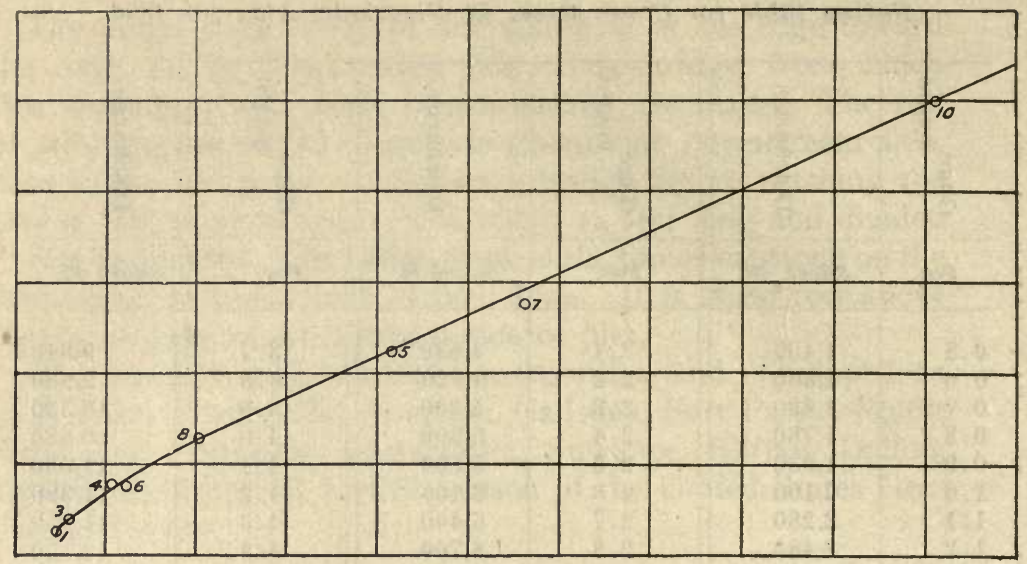

Fig. 8.- Rating curve for Riverside station on Coosa River, Ala.

Daily gage height, in feet, of Coosa River, at Riverside, Ala., for $189 \%$.

\begin{tabular}{|c|c|c|c|c|c|c|c|c|c|c|c|c|}
\hline Day & Jan. & Feb. & Mar. & April & May & Jun. & July & Aug. & Sept. & Oct. & Nov. & De \\
\hline & & 2.00 & 5.00 & 4.30 & 3.40 & 1.90 & 1.45 & 2.00 & 1.20 & 0.50 & 0.70 & \\
\hline & & & & & & 1.90 & & & & .50 & .70 & \\
\hline & & & & & & 1.90 & 1.4 & & & .50 & & \\
\hline & & & & 7. & & 1.85 & 1.40 & & & .50 & .65 & \\
\hline & & & & & & 1.85 & 1.45 & & & .45 & .80 & \\
\hline & & 7. & 5.8 & 9. & 3. & 1.80 & 1.50 & & & .45 & 1.05 & \\
\hline & & 9. & 11.4 & 10. & & 1.90 & & & & .45 & 1.15 & \\
\hline & 1.3 & 7.7 & 3.3 & 11. & 3 . & 2.15 & 2.40 & & 1. & .45 & 1.10 & \\
\hline & & 6. & 1 & 12. & & 2.10 & & & & .45 & & \\
\hline & 1.3 & 5. & 12. & 11. & & 1.90 & 2. & & 35 & .45 & .95 & \\
\hline & 1.3 & 5.2 & $12 . ?$ & 10. & & 1.90 & & & & .40 & .8 & \\
\hline & 1.3 & 7.35 & 12.8 & 9. & & 2.00 & & & .8 & .40 & .85 & \\
\hline & & 8. & 13. & & & 1. & 2. & & .7 & .45 & .8 & \\
\hline & & 8. & 14. & 6. & & .85 & 2. & & 7 & .45 & .85 & \\
\hline & & 7. & 14. & & & 0 & & & .8 & 1.45 & .8 & \\
\hline & & 6 & 14.8 & & & & & & 8 & 1.65 & .75 & \\
\hline & & & 14. & & & & 1. & & .8 & 1.40 & .70 & \\
\hline & 5. & 5.0 & 14. & 5. & & & & & .80 & 1.35 & .70 &. \\
\hline & & & 14. & & & & 2. & & .8 & 1.20 & .7 & \\
\hline & 4.8 & & 15. & 4. & & & & & .7 & 1.00 & .65 & 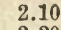 \\
\hline & & & 14. & & & & & & .7 & .90 & .65 & \\
\hline & 7. & & 14. & 4. & & & & & .7 & .85 & .65 & 2. \\
\hline & 7.3 & & 14. & & 2.3 & 1.60 & & & .7 & .80 & .6 & \\
\hline & 7.0 & 7.9 & 13. & 3. & & & 6. & & .7 & .70 & .65 & 4. \\
\hline & 5.4 & 9.00 & 12.2 & & 2.25 & 1.50 & & & & .80 & .65 & \\
\hline & $4 . ?$ & 9.00 & 10. & 3. & 2.15 & & 4. & & .6 & .75 & .65 & 4. \\
\hline & 3.80 & 8.00 & & 3.25 & & & & & .6 & .60 & .65 & \\
\hline & 3.00 & 6.20 & 6.5 & 2.20 & 2.0 & & 2. & & .5 & .65 & .65 & 3.2 \\
\hline & & & & 3.10 & & 1.4 & & & .55 & .80 & .70 & 2. \\
\hline & 2.50 & & 4.9 & 3.20 & 1.95 & 1.45 & 3.00 & 1.35 & .55 & .75 & .75 & 2.8 \\
\hline & 2.20 & $\cdots \cdots$ & 4.60 & ....... & 1.90 & & 2.60 & 1.30 & & .70 & & 2.5 \\
\hline
\end{tabular}


Rating table for Coosa River at Riverside, Ala., for 189\%.

\begin{tabular}{|c|c|c|c|c|c|}
\hline 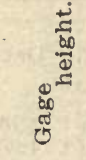 & 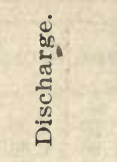 & 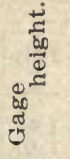 & 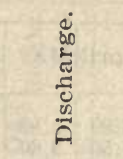 & 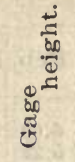 & 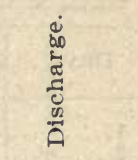 \\
\hline Feet. & Second $F t$. & Feet. & Second $F t$. & Feel. & Second $F t$. \\
\hline 0.4 & 1,350 & 2.0 & 4,520 & 5.0 & 14,046 \\
\hline 0.5 & 1,400 & 2.2 & 5,100 & 6.0 & 17,306 \\
\hline 0.6 & 1,500 & 2.4 & 5,700 & 7.0 & 20,566 \\
\hline 0.7 & 1,650 & 2.6 & 6,300 & 8.0 & 23,826 \\
\hline 0.8 & 1,820 & 2.8 & 6,910 & 9.0 & 27,086 \\
\hline 0.9 & 2,010 & 3.0 & 7,530 & 10.0 & 30,346 \\
\hline 1.0 & 2,210 & 3.2 & 8,178 & 11.0 & 33,606 \\
\hline 1.2 & 2,630 & 3.4 & 8,830 & 12.0 & 36,866 \\
\hline 1.4 & 3,070 & 3.6 & 9,482 & 13.0 & 40,126 \\
\hline 1.6 & 3,540 & 3.8 & 10,134 & 14.0 & 43,386 \\
\hline 1.8 & 4,020 & 4.0 & 10,786 & & \\
\hline
\end{tabular}

Note.-This table applied to the foregoing "Daily gage heights" gives the cubic feet per second flowing in the river on each date for which the gage height is given.

The following discharge measurements were made during I 898 by Max Hall and others : feet.

January 27: Gage height, 10.00 feet; discharge, 30,359 second-

March 9: Gage height, 1.60 feet; discharge, 3,538 second-feet.

May 3: Gage height, 3.22 feet; discharge, 7,758 second-feet.

May 25: Gage height, 1.39 feet; discharge, 3,172 second-feet.

August 3: Gage height, 3.92 feet; discharge, 9,524 second-feet. feet.

September 7: vage height, 11.05 feet; discharge, 37,811 second-

October 19: Gage height, 6.80 feet; discharge, 14,484 second-feet. November 22: Gage height, 5.85 feet; discharge, 16,384 second-feet. 
Daily gage height, in feet, of Coosa River at Riverside, Ala., for 1898.

\begin{tabular}{|c|c|c|c|c|c|c|c|c|c|c|c|c|}
\hline Day & Jan. & Feb. & Mar. & April & May & Jun. & July & Aug. & Sept. & Oct. & Nov. & Dec \\
\hline & & & 1.65 & & 3.90 & 1.30 & 1.15 & 4.25 & 2.20 & 2.20 & 2.60 & \\
\hline & & & & & & 1.25 & & & & & & \\
\hline & & & & & & 1.20 & & & & & & \\
\hline & & & & & 3 & 1.20 & .95 & & & & & \\
\hline & & & & & & 1.30 & .95 & & & & & \\
\hline & & & & 9.3 & & 1.25 & .90 & & 10. & & & \\
\hline & & & & 10.5 & & 1.20 & .95 & & 11. & & & 1. \\
\hline & 1 & 2. & 1. & 10. & 2 . & 1.10 & 1.00 & & 11. & & & 1.0 \\
\hline & & & & 10. & & 1.05 & 1.15 & & 11. & & & .7 \\
\hline & & 2. & & & 2. & 1.05 & 1. & & 10. & & & 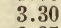 \\
\hline & & & & & & 1.00 & & & & & & \\
\hline & & 2. & & & 2. & 1.48 & & & & 11. & & \\
\hline & & & & & 2. & 1.10 & & & & & & 3. \\
\hline & & 2. & & & 1. & 1.25 & & & & & & \\
\hline & & & & & 1. & 1.15 & & & & & & 2. \\
\hline & & & & & 1. & 1.00 & & & & & & 2. \\
\hline & & & & & 1. & 1.65 & 0 & & & & & 2. \\
\hline & & 1. & & & 1. & 1.70 & & & & & & 2.6 \\
\hline & & 1. & & & 1. & 1.65 & 2. & & & & & 2. \\
\hline & & 1. & & & & & & & & & & \\
\hline & & & & & 1. & 1.95 & & & & & & 4. \\
\hline & & 1.8 & & & 1. & 2.10 & 1. & & & & & 3. \\
\hline & & & & & & 2. & & & & & & 3. \\
\hline & & & & & 1. & 2.5 & 1. & & & & & 3. \\
\hline & & & & & & 2.0 & & & & & & \\
\hline & 9. & & & & 1. & 1.75 & 1. & & & & & 2. \\
\hline & 10. & & & & & 1.5 & & & & & & 2. \\
\hline & 10. & 1.65 & & & 1. & 1.6 & & & & & & 2. \\
\hline & 10. & & & & & 1.45 & & & & & & 2. \\
\hline & 9.4 & & 3. & 4.75 & 1. & 1.30 & 3. & 3. & 2.75 & 2.75 & 3.80 & 2.3 \\
\hline & 7.55 & & 4.50 & & 1.35 & & 4.00 & 2.60 & & 2.70 & & 2.4 \\
\hline
\end{tabular}


Rating table for Coosa River at Riverside, Ala., for 189s.

\begin{tabular}{|c|c|c|c|c|c|c|c|}
\hline & 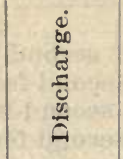 & 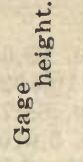 & 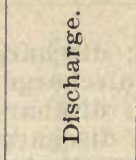 & 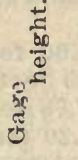 & 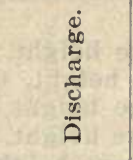 & 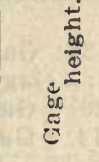 & 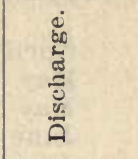 \\
\hline Feel. & Secind-feer & Feet. & Second-feet. & Feet & Second-feet. & Feet. & Seconil-feet. \\
\hline 0.9 & 2,140 & 4.7 & 12,301 & 8.5 & 25,335 & 12.3 & 46,960 \\
\hline 1.0 & 2,320 & 4.8 & 12,644 & 8.6 & 25,678 & 12.4 & 47,680 \\
\hline 1.1 & 2,520 & 4.9 & 12,987 & 8.7 & 26,021 & 12.5 & 48,400 \\
\hline 1.2 & 2,720 & 5.0 & 13,330 & 8.8 & 26,364 & 12.6 & 49,120 \\
\hline 1.3 & 2,925 & 5.1 & 13,673 & 8.9 & 26,707 & 12.7 & 49,840 \\
\hline 1.4 & 3,130 & 5.2 & 14,016 & 9.0 & 27,050 & 12.8 & 50,560 \\
\hline 1.5 & 3,340 & 5.3 & 14,359 & 9.1 & 27,433 & 12.9 & 51,280 \\
\hline 1.6 & 3,550 & 5.4 & 14,702 & 9.2 & 27,800 & 13.0 & 52,000 \\
\hline 1.7 & 3,760 & 5.5 & 15,045 & 9.3 & 28,175 & 13.1 & 52,720 \\
\hline 1.8 & 3,970 & 5.6 & 15,388 & 9.4 & 28,550 & 13.2 & 53,440 \\
\hline 1.9 & 4,185 & 5.7 & 15,731 & 9.5 & 28,965 & 13.3 & 54,160 \\
\hline 2.0 & 4,400 & 5.8 & 16,074 & 9.6 & 29,380 & 13.4 & 54,885 \\
\hline 2.1 & 4,620 & 5.9 & 16,417 & 9.7 & 29,815 & 13.5 & 55,600 \\
\hline 2.2 & 4,840 & 6.0 & 16,760 & 9.8 & 30,250 & 13.6 & 56,320 \\
\hline 2.3 & 5,070 & 6.1 & 17,103 & 9.9 & 30,725 & 13.7 & 57,040 \\
\hline 2.4 & 5,300 & 6.2 & 17,446 & 10.0 & 31,200 & 13.8 & 57,760 \\
\hline 2.5 & 5,540 & 6.3 & 17,789 & 10.1 & 31,725 & 13.9 & 58,480 \\
\hline 2.6 & 5,780 & 6.4 & 18,132 & 10.2 & 32,250 & 14.0 & 59,200 \\
\hline 2.7 & 6,030 & 6.5 & 18,475 & 10.3 & 32,825 & 14.1 & 59,920 \\
\hline 2.8 & 6,280 & 6.6 & 18,818 & 10.4 & 33,400 & 14.2 & טง, 640 \\
\hline 2.9 & 6,540 & 6.7 & 19,161 & 10.5 & 34,067 & 14.3 & 61,360 \\
\hline 3.0 & 6,800 & 6.8 & 19,540 & 10.6 & 34,725 & 814.4 & 62,080 \\
\hline 3.1 & 7,080 & 6.9 & 19,847 & 10.7 & 35,442 & 14.5 & 62,800 \\
\hline 3.2 & 7,360 & 7.0 & 20,190 & 10.8 & 36,160 & 14.6 & 63,520 \\
\hline 3.3 & 7,655 & 7.1 & 20,533 & 10.9 & 36,880 & 14.7 & 64,240 \\
\hline 3.4 & 7,950 & 7.2 & 20,876 & 11.0 & 37,600 & 14.8 & 64,960 \\
\hline 3.5 & 8,260 & 7.3 & 21,219 & 11.1 & 38,320 & 14.9 & 65,680 \\
\hline 3.6 & 8,570 & 7.4 & 21,562 & 11.2 & 39,040 & 15.0 & 66,400 \\
\hline 3.7 & 8,895 & 7.5 & 21,905 & 11.3 & $39, / 60$ & 15.1 & 67,120 \\
\hline 3.8 & 9,220 & 7.6 & 22,248 & 11.4 & 40,480 & 15.2 & 67,840 \\
\hline 3.9 & 9,560 & 7.7 & 22,591 & 11.5 & 41,200 & 15.3 & 68,560 \\
\hline 4.0 & 9,900 & 7.8 & 22,934 & 11.6 & 41,920 & 15.4 & 69,280 \\
\hline 4.1 & 12,243 & 7.9 & 23,277 & 11.7 & 42,640 & 15.5 & 70,000 \\
\hline 4.2 & 10,586 & 8.0 & 23,620 & 11.8 & 43,360 & 15.6 & 70,720 \\
\hline 4.3 & 10,929 & 8.1 & 23,963 & 11.9 & 44,080 & 15.7 & 71,440 \\
\hline 4.4 & 11,272 & 8.2 & 24,306 & 12.0 & 44,800 & 15.8 & 72,160 \\
\hline 4.5 & 11,615 & 8.3 & 24,649 & 12.1 & 45,520 & 15.9 & 72,880 \\
\hline 4.6 & 11,958 & 8.4 & 24,992 & 12.2 & 46,240 & 16.0 & 73,600 \\
\hline
\end{tabular}

Note.-This table applied to the foregoing "Daily gage heights" gives the cubic feet per second flowing in the river on each date for which the gage height is given.

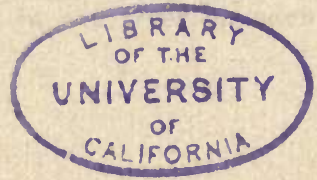


The following discharge measurements were made during I 899 by Max Hall and others :

April 26: Gage height, 9.00 feet; discharge, 29,069 second-feet.

May 3: Gage height, 4.05 feet; aischarge, 10,592 second-feet.

May 20: Gage height, 2.70 feet; discharge, 6,276 second-feet.

June 14: Gage height, 2.20 feet; discharge, 5,010 second-feet.

August 26: Gage height, 1.42 feet; discharge, 3,791 second-feet. feet.

September 23: Gage height, 1.00 foot; discharge, 2,457 second-

November 7: Gage height, 0.85 foot; discharge, 2,271 second-feet.

December 9: vage height, 1.20 feet; discharge, 2,727 second-feet.

Daily gage height, in feet, of Coosa River at Riverside, Ala., for 1899.

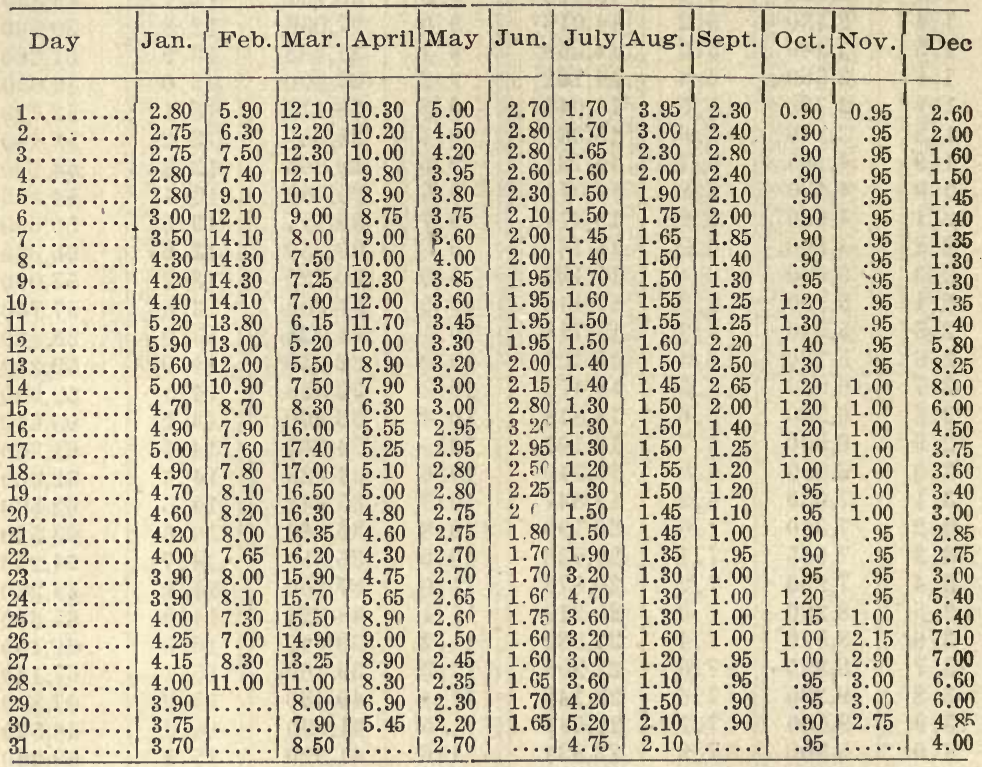


Rating table for Coosa river at Riverside, Ala., for 1899.

\begin{tabular}{|c|c|c|c|c|c|c|c|}
\hline 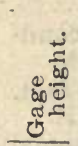 & 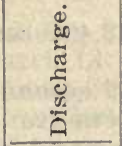 & 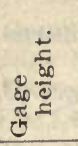 & 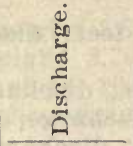 & 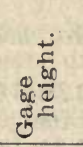 & 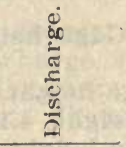 & 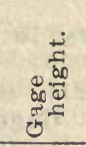 & 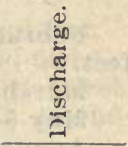 \\
\hline Feet. & Second $f$. & Feet. & Second feet. & Feet. & Second feet. & Feet. & Second feet \\
\hline 0.9 & 2,330 & 5.2 & 14,740 & 9.5 & 30,650 & 13.8 & 46,560 \\
\hline 1.0 & 2,460 & 5.3 & 15,110 & 9.6 & 31,020 & 13.9 & 46,930 \\
\hline 1.1 & 2,600 & 5.4 & 15,480 & 9.7 & 31,390 & 14.0 & 47,300 \\
\hline 1.2 & 2,760 & 5.5 & 15,850 & 9.8 & 31,760 & 14.1 & 47,670 \\
\hline 1.3 & 2,920 & 5.6 & 16,220 & 9.9 & 32,130 & 14.2 & 48,040 \\
\hline 1.4 & 3,100 & 5.7 & 16,590 & 10.0 & 32,500 & 14.3 & 48,410 \\
\hline 1.5 & 3,300 & 5.8 & 16,960 & 10.1 & 32,870 & 14.4 & 48,780 \\
\hline 1.6 & 3,500 & 5.9 & 17,330 & 10.2 & 33,240 & 14.5 & 49,150 \\
\hline 1.7 & 3,720 & 6.0 & 17,700 & 10.3 & 33,610 & 14.6 & 49,520 \\
\hline 1.8 & 3,940 & 6.1 & 18,070 & 10.4 & 33,980 & 14.7 & 49,890 \\
\hline 1.9 & 4,160 & 6.2 & 18,440 & 10.5 & 34,350 & 14.8 & 50,260 \\
\hline 2.0 & 4,400 & 6.3 & 18,810 & 10.6 & 34,720 & 14.9 & 50,630 \\
\hline 2.1 & 4,600 & 6.4 & 19,180 & 10.7 & 35,090 & 15.0 & 51,000 \\
\hline 2.2 & 4,900 & 6.5 & 19,550 & 10.8 & 35,460 & 15.1 & 51,370 \\
\hline 2.3 & 5,160 & 6.6 & 19,920 & 10.9 & 35,830 & 15.2 & 51,740 \\
\hline 2.4 & 5,430 & 6.7 & 20,290 & 11.0 & 36,200 & 15.3 & 52,110 \\
\hline 2.5 & 5,700 & 6.8 & 20,660 & 11.1 & 36,570 & 15.4 & 52,480 \\
\hline 2.6 & 5,970 & 6.9 & 21,030 & 11.2 & 36,940 & 15.5 & 52,850 \\
\hline 2.7 & 6,250 & 7.0 & 21,400 & 11.3 & 37,310 & 15.6 & 53,220 \\
\hline 4.8 & 6,530 & 7.1 & 21,170 & 11.4 & 37,680 & 15.7 & 53,590 \\
\hline 2.9 & 6,810 & 7.2 & 22,140 & 11.5 & 38,050 & 15.8 & 53,960 \\
\hline 3.0 & 7,100 & 7.3 & 22,510 & 11.6 & 38,420 & 15.9 & 54,330 \\
\hline 3.1 & 7,400 & 7.4 & 22,880 . & 11.7 & 38,790 & 16.0 & 54,700 \\
\hline 3.2 & 7,700 & 7.5 & 23,250 & 11.8 & 39,160 & 16.1 & 55,070 \\
\hline 3.3 & 8,010 & 7.6 & 23,620 & 11.9 & 39,530 & 16.2 & 55,440 \\
\hline 3.4 & 8,330 & 7.7 & 23,990 & 12.0 & 39,900 & 16.3 & 55,810 \\
\hline 3.5 & 8,650 & 7.8 & 24,360 & 12.1 & 40,270 & 16.4 & 56,280 \\
\hline 3.6 & 8,970 & 7.9 & 24,730 & 12.2 & 40,640 & 16.5 & 56,650 \\
\hline 3.7 & 9,290 & 8.0 & 25,100 & 12.3 & 41,010 & 16.6 & 57,020 \\
\hline 3.8 & 9,620 & 8.1 & 25,470 & 12.4 & 41,380 & 16.7 & 57,390 \\
\hline 3.9 & 9,950 & 8.2 & 25,840 & 12.5 & 41,750 & 16.8 & 57,760 \\
\hline 4.0 & 10,300 & 8.3 & 26,210 & 12.6 & 42,120 & 16.9 & 58,130 \\
\hline 4.1 & 10,670 & 8.4 & 26,580 & 12.7 & 42,490 & 17.0 & 58,400 \\
\hline 4.2 & 11,040 & 8.5 & 26,950 & 12.8 & 42,860 & 17.1 & 58,770 \\
\hline 4.3 & $11,4 \perp \cup$ & 8.6 & 27,320 & 12.9 & 43,230 & 17.2 & 59,140 \\
\hline 4.4 & 11,780 & 8.7 & 27,690 & 13.0 & 43,600 & 17.3 & 59,510 \\
\hline 4.5 & 12,150 & 8.8 & 28,060 & 13.1 & 43,970 & 17.4 & 59,880 \\
\hline 4.6 & 12,520 & 8.9 & 28,430 & 13.2 & 44,340 & 17.5 & 60,250 \\
\hline 4.7 & 12,890 & 9.0 & 28,800 & 13.3 & 44,710 & 17.6 & 60,620 \\
\hline 4.8 & 13,260 & 9.1 & 29,170 & 13.4 & 45,080 & 17.7 & 60,990 \\
\hline 4.9 & 13,630 & 9.2 & 29,540 & 15.5 & 45,450 & 17.8 & 61,360 \\
\hline 5.0 & 14,000 & 9.3 & 29,910 & 13.6 & 45,720 & 17.9 & 61,730 \\
\hline 5.1 & 14,370 & 9.4 & 30,280 & 13.7 & 46,190 & 18.0 & 62,100 \\
\hline
\end{tabular}

NotE.-This table applied to the foregoing "Daily gage heights" gives the cubic feet per second flowing in the river on each date for which the gage height is given. 
The following discharge measurements were made during I900 by Max Hall and others : feet.

February 10: Gage height, 5.03 feet; discharge, 13,493 second-

March 21: Gage height, 12.50 feet; discharge, 43,759 second-feet.

May 5: Gage height, 4.15 feet; discharge, 11,196 second-feet.

August 21: Gage height, 2.32 feet; discharge, 5,609 second-feet. feet.

December 28: Gage height, 4.25 feet; discharge, 11,335 second-

Daily gage height, in feet, of Coosa River, near Riverside, Ala.. for 1900 .

\begin{tabular}{|c|c|c|c|c|c|c|c|c|c|c|c|c|}
\hline Day & Jan. & Feb. & Mar. & April & May & Jun. & July & Aug. & Sept. & Oct & & D) \\
\hline & & 2,70 & 6.90 & 6.65 & 5.00 & 2.75 & 11.60 & .70 & 1.85 & 1.55 & 2.30 & 0.75 \\
\hline & & & & & & & & & & & & \\
\hline & & & & & & 2.90 & & & & & & \\
\hline & & & 6. & & & 2.60 & & 2.75 & 2.10 & & & \\
\hline & & & 5. & 5.1 & & 2.70 & 7. & & 2.0 & & & \\
\hline & & & 5.0 & 4. & 20 & 3.45 & & & 1.8 & & 10 & \\
\hline & 1.9 & 2.9 & 4.9 & 4. & & 3.90 & & & & & & \\
\hline & 1. & 3.0 & 6.0 & 4. & & 4.20 & 4. & 2.15 & 1.6 & & 2. & \\
\hline 9. & 2.0 & & 8.7 & 4. & & $7.1^{\circ}$ & 5. & 2.10 & 1.5 & & & \\
\hline & & & 10.0 & 4. & & 8.30 & & & 1. & & & \\
\hline & & & 10.5 & 6. & & 8.00 & & & & & & \\
\hline & & & 10.0 & 12. & & 7.70 & 4. & 1.90 & 1. & & & 3. \\
\hline & 6.0 & 13.3 & 8.7 & 12. & & $6.7 r$ & & 2.25 & & & & \\
\hline & & & & 11.7 & & 4.30 & & 2.00 & & & & \\
\hline & 7.0 & 15. & 5. & 9. & & 4.50 & & 1. & & & & \\
\hline & 6.4 & 14. & 6. & 7.2 & & 4.70 & & 1. & & & & 2.6 \\
\hline & 5.10 & 14. & 6.3 & 12.4 & & 5.00 & & & & & & \\
\hline & 4.0 & & 6. & & & 4. & & & & & & \\
\hline & 4.2 & 12.8 & 6.5 & 17. & & 6.90 & & & & & & \\
\hline & 8.0 & 12.1 & & & & 6.90 & & & & & & \\
\hline & 9.7 & 9.0 & 12. & & & 6.45 & 2. & & & & & \\
\hline & 10.0 & & 12.8 & 13. & & 6.10 & & & & & & \\
\hline & 9.40 & 6. & 12.6 & 12. & 2. & $7 . r n$ & & & & & & \\
\hline & & & 11.8 & 12.2 & & 11.35 & & & & & & \\
\hline & & 6.9 & 10.6 & 10.8 & 3. & 112.50 & & & & & & \\
\hline & 6.0 & & & & 3. & $14.1 \mathrm{f}$ & & & & & & \\
\hline & 4.1 & 5.2 & 10.2 & 7. & 3. & $114 ?$ & 2. & 2. & & & 4.5 & \\
\hline & & 5.00 & 9. & 6.5 & 3. & |14.60 & & & & & & \\
\hline & 3. & & 9. & 5. & 2 . & 13.80 & 5. & & 1. & & 9.20 & 7.0 \\
\hline & 3.0 & & 8.5 & 5.35 & 2 . & $1 \div 8$ & & 1.90 & 1.55 & & 8.20 & 4.0 \\
\hline & 2.70 & & 7.2 & & & & 5.90 & 1.90 & ...... & 2.50 & ....... & 6.5 \\
\hline
\end{tabular}


The following discharge measurements were made during I9or by Max Hall and others:

January 8: Gage height, 3.85 feet; discharge, 9,572 second-feet. March 18: Gage height, 3.70 feet; discharge, 9,333 second-feet.

August 24: Gage height, 12.95 feet ; discharge, 44,554 second-feet. feet.

November 14: Gage height, 1.70 feet; discharge, 4,039 second-

Daily gage height, in feet, of Coosa River, at Riverside, Ala., for 1901.

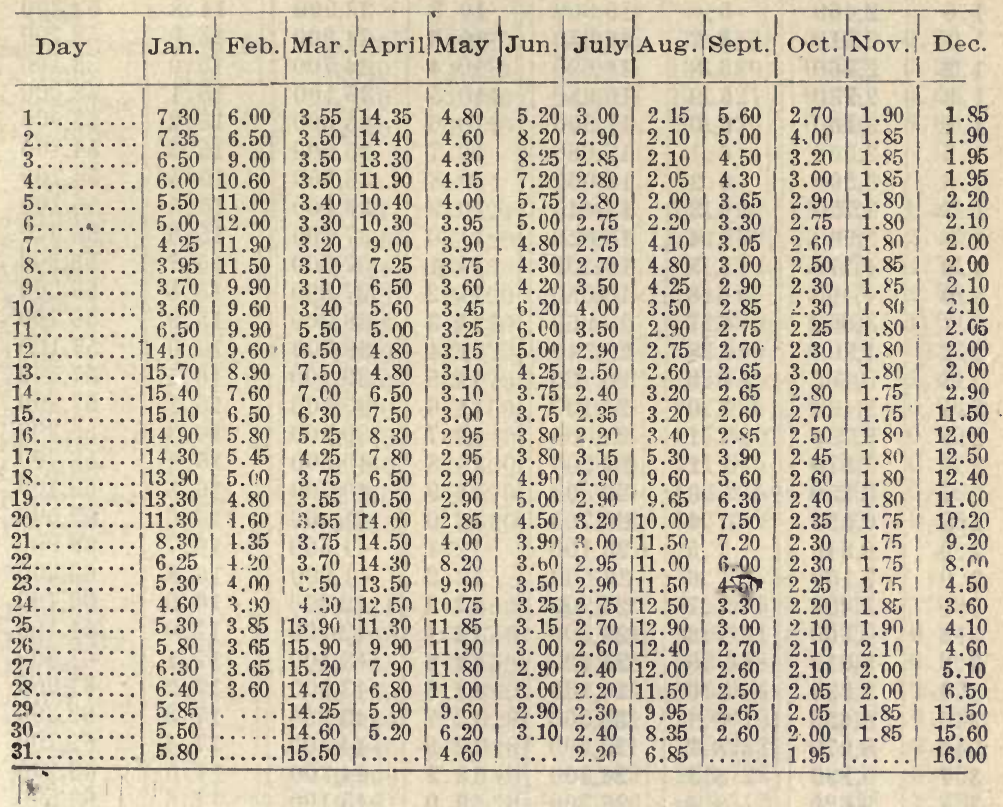


Rating table for Coosa River at Riverside, Ala., for 1900 and 1901.

\begin{tabular}{|c|c|c|c|c|c|c|c|}
\hline 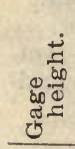 & 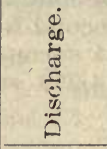 & 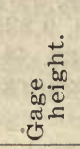 & 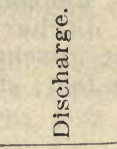 & 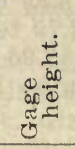 & 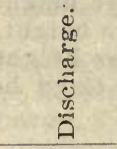 & 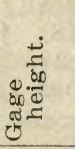 & 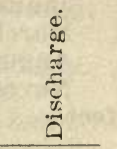 \\
\hline Feet. & Second-ft. & Feet. & Second $f l$. & Feet. & Second-ft. & Feet. & Second-ft. \\
\hline 1.0 & 2,460 & 5.6 & 15,900 & 10.2 & 33,900 & 14.8 & 52,300 \\
\hline 1.1 & 2,610 & 5.7 & 16,250 & 10.3 & 34,300 & 14.9 & 52,700 \\
\hline 1.2 & 2,760 & 5.8 & 16,600 & 10.4 & 34,700 & 15.0 & 53,100 \\
\hline 1.3 & 2,930 & 5.9 & 16,950 & 10.5 & 35,100 & 15.1 & 53,500 \\
\hline 1.4 & 3,100 & 6.0 & 17,300 & 10.6 & 35,500 & 15.2 & 53,900 \\
\hline 1.5 & 3,300 & 6.1 & 17,680 & 10.7 & 35,900 & 15.3 & 54,300 \\
\hline 1.6 & 3,500 & 6.2 & 18,060 & 10.8 & 36,300 & 15.4 & 54,700 \\
\hline 1.7 & 3,720 & 6.3 & 18,440 & 10.9 & 36,700 & 15.5 & 55,100 \\
\hline 1.8 & 3,940 & 6.4 & 18,820 & 11.0 & 37,100 & 15.6 & 55,500 \\
\hline 1.9 & 4,170 & 6.5 & 19,200 & 11.1 & 37,500 & 15.7 & 55,900 \\
\hline 2.0 & 4,400 & 6.6 & 19,580 & 11.2 & 37,900 & 15.8 & $56: \therefore 00$ \\
\hline 2.1 & 4,650 & 6.7 & 19,960 & 11.3 & 38,300 & 15.9 & 56,700 \\
\hline 2.2 & 4,900 & 6.8 & 20,340 & 11.4 & 38,700 & 16.0 & 57,100 \\
\hline 2.3 & 5,165 & 6.9 & 20,720 & 11.5 & 39,100 & 16.1 & 57,500 \\
\hline 2.4 & 5,430 & 7.0 & 21,100 & 11.6 & 39,500 & 16.2 & 57,900 \\
\hline 2.5 & 5,700 & 7.1 & 21,500 & 11.7 & 39,900 & 16.3 & ๖8,300 \\
\hline 2.6 & 5,970 & 7.2 & 21,900 & 11.8 & 40,300 & 16.4 & 58,700 \\
\hline 2.7 & 6,250 & 7.3 & 22,300 & 11.9 & 40,700 & 16.5 & 59,100 \\
\hline 2.8 & 6,530 & 7.4 & 22,700 & 12.0 & 41,100 & 16.6 & 59,500 \\
\hline 2.9 & 6,845 & 7.5 & 23,100 & 12.1 & 41,500 & 16.7 & 59,900 \\
\hline 3.0 & 7,100 & 7.6 & 23,500 & 12.2 & 41,900 & 16.8 & 60,300 \\
\hline 3.1 & 7,400 & 7.7 & 23,900 & 12.3 & 42,300 & 16.9 & 60,700 \\
\hline 3.2 & 7,700 & 7.8 & 24,300 & 12.4 & 42,700 & 17.0 & 61,100 \\
\hline 3.3 & 8,015 & 7.9 & 24,700 & 12.5 & 43,100 & 17.1 & 61,500 \\
\hline 3.4 & 8,330 & 8.0 & 25,100 & 12.6 & 43,500 & 17.2 & 61,900 \\
\hline 3.5 & 8,650 & 8.1 & 25,500 & 12.7 & 43,900 & 17.3 & 62,300 \\
\hline 3.6 & 8,970 & 8.2 & 25,900 & 12.8 & 44,300 & 17.4 & 62,700 \\
\hline 3.7 & 9,295 & 8.3 & 26,300 & 12.9 & 44,700 & 17.5 & 63,100 \\
\hline 3.8 & 9,620 & 8.4 & 26,700 & 13.0 & 45,100 & 17.6 & 63,500 \\
\hline 3.9 & 9,960 & 8.5 & 27,100 & 13.1 & 45,500 & 17.7 & 63,900 \\
\hline 4.0 & 10,300 & 8.6 & 27,500 & 13.2 & 45,900 & 17.8 & 64,300 \\
\hline 4.1 & 10,650 & 8.7 & 27,900 & 13.3 & 46,300 & 17.9 & 64,700 \\
\hline 4.2 & 11,000 & 8.8 & 28,300 & 13.4 & 46,700 & 18.0 & 65,100 \\
\hline 4.3 & 11,350 & 8.9 & 28,700 & 13.5 & 47,100 & 18.1 & 65,500 \\
\hline 4.4 & 11,700 & 9.0 & 29,100 & 13.6 & 47,500 & 18.2 & 65,900 \\
\hline 4.5 & 12,050 & 9.1 & 29,500 & 13.7 & 47,900 & 18.3 & 66,300 \\
\hline 4.6 & 12,400 & 9.2 & 29,900 & 13.8 & 48,300 & 18.4 & 66,700 \\
\hline 4.7 & 12,750 & 9.3 & 30,300 & 13.9 & 48,700 & 18.5 & 67,100 \\
\hline 4.8 & 13,100 & 9.4 & 30,700 & 14.0 & 49,100 & 18.6 & 67,500 \\
\hline 4.9 & 13,450 & 9.5 & 31,100 & 14.1 & 49,500 & 18.7 & 67,900 \\
\hline 5.0 & 13,800 & 9.6 & 31,500 & 14.2 & 49,900 & 18.8 & 68,300 \\
\hline 5.1 & 14,150 & 9.7 & 31,900 & 14.3 & 50,300 & 18.9 & 68.700 \\
\hline 5.2 & 14,500 & 9.8 & 32,300 & 14.4 & 50,700 & 19.0 & 69,100 \\
\hline 5.3 & 14,850 & 9.9 & 32,700 & 14.5 & 51,100 & & \\
\hline 5.4 & 15,200 & 10.0 & 33,100 & 14.6 & 51,500 & & \\
\hline 5.5 & 15,550 & 10.1 & 33,500 & 14.7 & 51,900 & & \\
\hline
\end{tabular}

NotE.-This table applied to the foregoing "Daily gage heights" gives the cubic feet per second flowing in the river on each date for which the gage height is given. 
Estimated monthly discharge of Coosa River at Riverside, Ala.

[Drainage area, 6,850 square miles.]

\begin{tabular}{|c|c|c|c|c|c|c|}
\hline \multirow[b]{2}{*}{ Month. } & \multicolumn{3}{|c|}{ Discharge in second-feet. } & \multirow[b]{2}{*}{$\begin{array}{l}\text { Total in } \\
\text { acre- } \\
\text { feet. }\end{array}$} & \multicolumn{2}{|c|}{ Run-off. } \\
\hline & $\begin{array}{l}\text { Maxi- } \\
\text { mum. }\end{array}$ & $\begin{array}{l}\text { Mini- } \\
\text { mum. }\end{array}$ & Mean. & & $\begin{array}{l}\text { Depth } \\
\text { in } \\
\text { inches. }\end{array}$ & $\begin{array}{c}\text { Second. } \\
\text { feet per } \\
\text { square } \\
\text { mile. }\end{array}$ \\
\hline 1896. & & & & & & \\
\hline September 27 to 30 & 1,400 & 1,350 & 1,363 & 10,812 & 0.03 & 0.20 \\
\hline October...$\ldots \ldots$ & 7,640 & 1,450 & 2,218 & 136,380 & 0.37 & 0.32 \\
\hline November $\ldots . . \ldots$ & 15,100 & 2,190 & 4,637 & 275,921 & 0.75 & 0.68 \\
\hline December & 12,110 & 2,280 & 4,125 & 253,636 & 0.69 & 0.60 \\
\hline 1897. & & & & & & \\
\hline January & 21,707 & 2,420 & 8,434 & 518,590 & 1.42 & 1.23 \\
\hline February ......... & 27,086 & 4,520 & 18,658 & $1,036,230$ & 2.83 & 2.72 \\
\hline March $\ldots \ldots \ldots \ldots$ & 47,624 & 10,460 & 32,481 & $1,997,180$ & 5.47 & 4.74 \\
\hline April $\ldots \ldots \ldots$. & 37,355 & 5,100 & 17,698 & $1,053,105$ & 2.87 & 2.58 \\
\hline May .. & 10,786 & 4,270 & 7,040 & 432,875 & 1.19 & 1.03 \\
\hline June & 4,950 & 3,0 & 3,915 & 232,960 & 0.63 & 0.57 \\
\hline July .. & 23,826 & 3,070 & 7,142 & 439,145 & 1.20 & 1.04 \\
\hline August ....... & 6,300 & 2,850 & 3,870 & 237,960 & 0.64 & 0.56 \\
\hline September ........ & 3,040 & 1,4 & 1,976 & 117,580 & 0.32 & 0.29 \\
\hline October .......... & 3,66 & 1,3 & 1,819 & 111,845 & 0.31 & 0.27 \\
\hline Novemwer ... & 2,525 & 1,570 & 1,786 & 106,275 & 0.29 & 0.26 \\
\hline December ........ & 13,883 & 1,820 & 6,566 & 403,730 & 1.10 & 0.96 \\
\hline 1898. & & & & & & \\
\hline January . & 35,084 & 3,550 & 11,572 & 711,539 & 1.95 & 1.69 \\
\hline February .... & 16,760 & 3,655 & 5,763 & 320,161 & 0.87 & 0.84 \\
\hline March $\ldots \ldots \ldots$ & 15,045 & 3,550 & 5,852 & 359,828 & 0.68 & 0.59 \\
\hline April $\ldots \ldots \ldots$ & 36,1 & 8,2 & 18,133 & $1,078,986$ & 2.95 & 2.65 \\
\hline May $\ldots \ldots \ldots$. & 9,560 & 3,0 & 4,684 & 288,010 & 0.78 & 0.68 \\
\hline June $\ldots$ & 5,540 & 2,320 & 3,281 & 195,233 & 0.54 & 0.48 \\
\hline July ... & 9,90 & & 4,289 & 722 & 0 . & 0.6 \\
\hline August ... & 19,16 & 3,9 & 8,758 & 538,512 & 1.48 & 1.28 \\
\hline September ... & 41,920 & 3,865 & 13,927 & 828,712 & 2.26 & 2.03 \\
\hline October .... & 72,1 & 3,7 & 19,936 & $1,225,825$ & 3.36 & 2.91 \\
\hline November .. & 20,190 & 5,3 & 8,375 & 498,345 & 1.36 & 1.22 \\
\hline December .. & 9,900 & 5,070 & 7,376 & 453,535 & 1.25 & 1.08 \\
\hline 1899. & & & & & & \\
\hline January & 17,330 & 6,390 & 10,865 & 668,063 & 1.78 & 1.54 \\
\hline February & 48,410 & 17,3 &, 974 & 1,72 & 4.56 & \\
\hline March ........ & 60,880 & 14,740 & 38,094 & $2,342,309$ & 6.21 & 5.39 \\
\hline April $\ldots \ldots \ldots$ & 41,010 & 11,410 & 24,915 & $1,482,545$ & 3.94 & 3.53 \\
\hline May ..... & 14,000 & & 7,742 & 476,037 & 1.27 & 1.10 \\
\hline June ......... & 7,700 & 3,500 & 4,771 & 283,894 & 0.75 & 0.68 \\
\hline July ......... & 14,74 이 & 2,760 & 5,318 & 326,991 & 0.86 & 0.75 \\
\hline August $\ldots . .$. . & 10,125 & & 3,806 & 234,022 & 0.62 & 0.54 \\
\hline September .. & 6,530 & 2,3 & 3,555 & 211,537 & 0.56 & 0.50 \\
\hline October ........ & 3,100 & 2,330 & 2,510 & 154,334 & 0.41 & 0.36 \\
\hline November ..... & 7,100 & & 3,086 & 183,630 & 0.49 & 0.44 \\
\hline December ......... & 26,025 & 2,920 & 10,631 & 653,675 & 1.73 & 1.50 \\
\hline
\end{tabular}


Estimated monthly discharge of Coosa River near Riverside, Ala.

[Drainage area, 7,065 square miles.]

\begin{tabular}{|c|c|c|c|c|c|c|}
\hline \multirow[b]{2}{*}{ Month } & \multicolumn{3}{|c|}{ Discharge in second-feet. } & \multirow[b]{2}{*}{$\begin{array}{l}\text { Total in } \\
\text { acre- } \\
\text { feet. }\end{array}$} & \multicolumn{2}{|c|}{ Run-off. } \\
\hline & $\begin{array}{l}\text { Maxi- } \\
\text { mum. }\end{array}$ & $\begin{array}{l}\text { Mini- } \\
\text { mum. }\end{array}$ & Mean. & & $\begin{array}{l}\text { Depth } \\
\text { in } \\
\text { inches. }\end{array}$ & $\begin{array}{l}\text { Second } \\
\text { feet per } \\
\text { square } \\
\text { mile. }\end{array}$ \\
\hline $\begin{array}{c}1900 . \\
\text { January }\end{array}$ & 33,100 & 4,280 & 13,344 & 820,491 & 2.18 & 1.89 \\
\hline February ....... & 54,300 & 5,700 & 23,487 & $1,304,402$ & 3.45 & 3.32 \\
\hline March $\ldots \ldots \ldots$ & 44,500 & 13,450 & 26,822 & $1,649,221$ & 4.38 & 3.80 \\
\hline April & 65,500 & 11,350 & 29,813 & $1,773,997$ & 4.71 & 4.22 \\
\hline May $\ldots \ldots . . .$. & 14,850 & 5,970 & - 8,198 & 504,075 & 1.34 & 1.16 \\
\hline June $\ldots \ldots$...... & 51,500 & 5,970 & 22,216 & $1,321,944$ & 3.51 & 3.14 \\
\hline July $\ldots \ldots . \ldots$ & 39,500 & 5,565 & 13,610 & 836,846 & 2.23 & 1.93 \\
\hline August $\ldots \ldots \ldots \ldots$ & 12,750 & 4,050 & 5,147 & 316,477 & 0.84 & 0.73 \\
\hline September $\ldots .$. & 23,100 & 2,760 & 6,483 & 385,765 & 1.03 & 0.92 \\
\hline October $\ldots \ldots$..... & 23,100 & 3,100 & 6,910 & 424,879 & 1.13 & 0.98 \\
\hline November $\ldots . .$. & 29,900 & $3,720^{-}$ & 7,673 & 456,575 & 1.22 & 1.09 \\
\hline December...... & 22,300 & 5,835 & 11,773 & $|723,894|$ & 1.93 & 1.67 \\
\hline The year $\ldots . .$. & 65,500 & 2,760 & 14,623 & $|10518566|$ & 27.95 & 2.07 \\
\hline
\end{tabular}

\begin{tabular}{|c|c|c|c|c|c|}
\hline \multirow[b]{2}{*}{ Month. } & \multicolumn{3}{|c|}{ Discharge in second-feet } & \multicolumn{2}{|c|}{ Run-off. } \\
\hline & $\begin{array}{l}\text { Maxi- } \\
\text { mum. }\end{array}$ & $\begin{array}{l}\text { Mini- } \\
\text { mum. }\end{array}$ & Mean. & $\begin{array}{l}\text { Depth } \\
\text { in } \\
\text { inches. }\end{array}$ & $\begin{array}{l}\text { Second. } \\
\text { feet per } \\
\text { square } \\
\text { mile. }\end{array}$ \\
\hline 1901. & & & & & \\
\hline January $\ldots \ldots \ldots \ldots$ & 55,900 & 8,970 & 26,089 & 4.25 & 3.69 \\
\hline February $\ldots \ldots \ldots \ldots \ldots$ & 41,100 & 8,970 & 21,784 & 3.21 & 3.08 \\
\hline 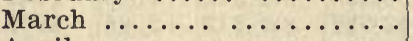 & 56,700 & 7,400 & 20,613 & 3.37 & 2.92 \\
\hline April $\ldots \ldots \ldots \ldots \ldots \ldots$ & 51,100 & 14,500 & 30,616 & 4.83 & 4.33 \\
\hline May $\ldots \ldots \ldots \ldots \ldots$ & 40,700 & 6,670 & 16,195 & 2.64 & 2.29 \\
\hline June $\ldots \ldots \ldots \ldots \ldots$ & 26,100 & 6,810 & 12,335 & 1.95 & 1.75 \\
\hline July $\ldots \ldots \ldots \ldots \ldots \ldots$ & 10,300 & 4,900 & 6,535 & 1.07 & 0.93 \\
\hline August $\ldots \ldots \ldots \ldots \ldots$ & 44,700 & 4,400 & 20,370 & 3.32 & 2.88 \\
\hline September ............. & 23,100 & 5,700 & 9,977 & 1.57 & 1.41 \\
\hline October $\ldots \ldots \ldots \ldots \ldots$ & 10,300 & 4,280 & 5,694 & 0.93 & 0.81 \\
\hline November $\ldots \ldots \ldots \ldots \ldots$ & 4,650 & 3,830 & 4,016 & 0.64 & 0.57 \\
\hline December $\ldots . . . . . . .$. & 57,100 & 4,050 & 18,885 & 3.08 & 2.67 \\
\hline The year $\ldots \ldots \ldots \ldots \ldots$ & 57,100 & 3,830 & 16,092 & 30.86 & 2.28 \\
\hline
\end{tabular}




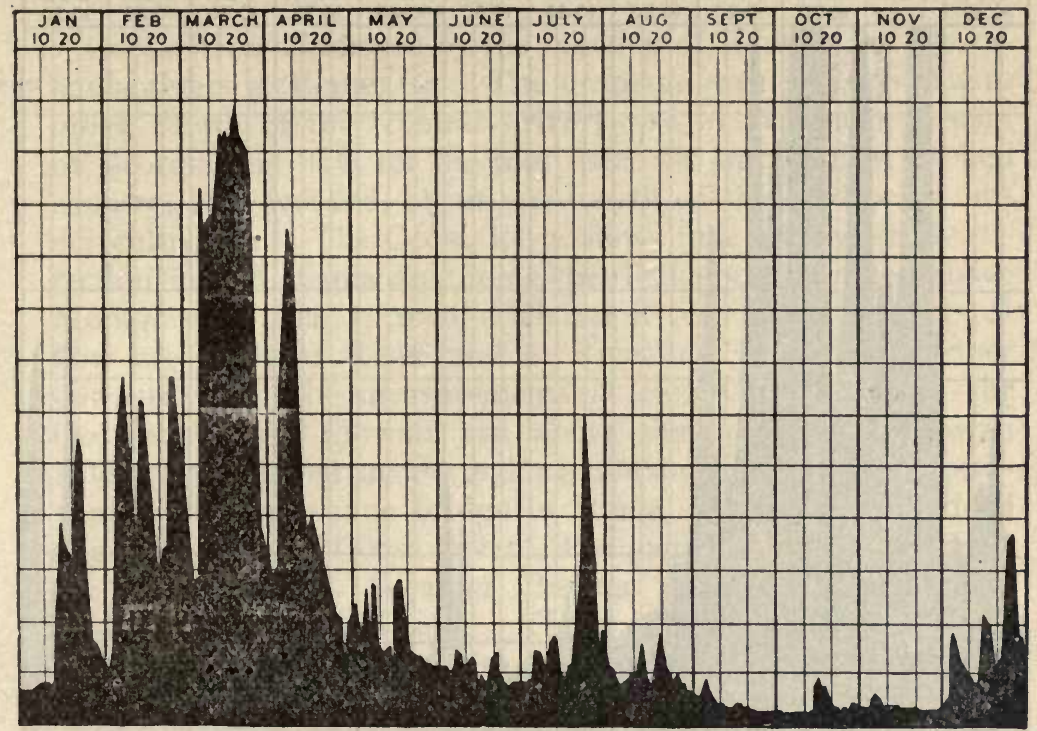

Fig. 9.-Discharge of Coosa River at Riverside, Ala., 1897.

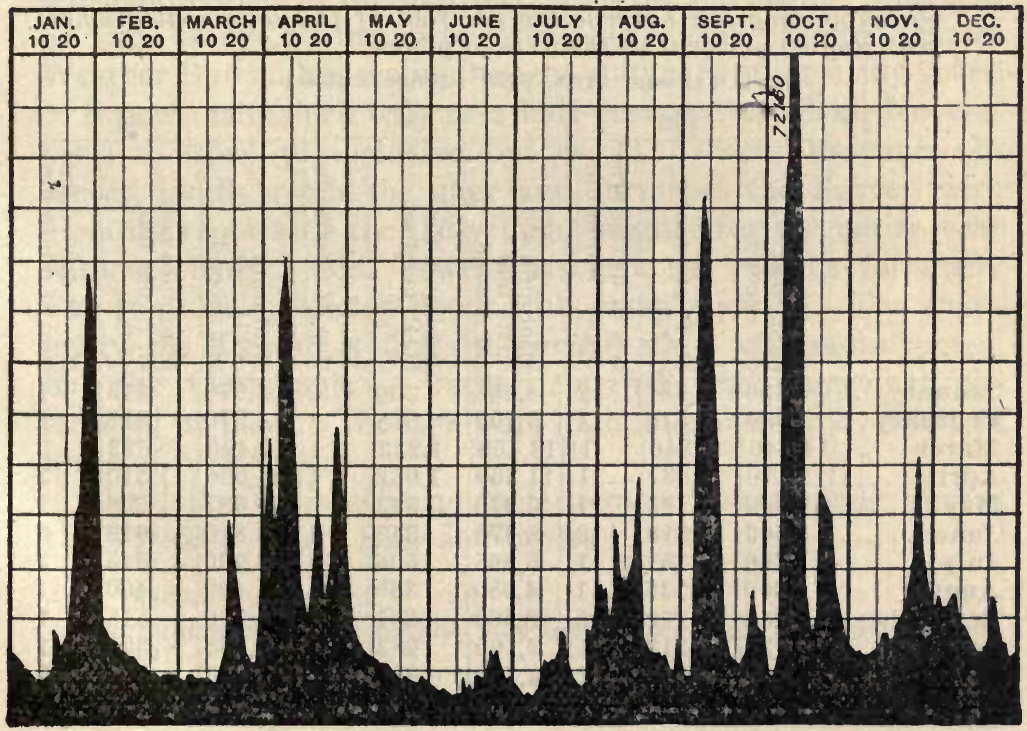

Fig. 10.-Discharge of Coosa River at Riverside, Ala., 1898. 


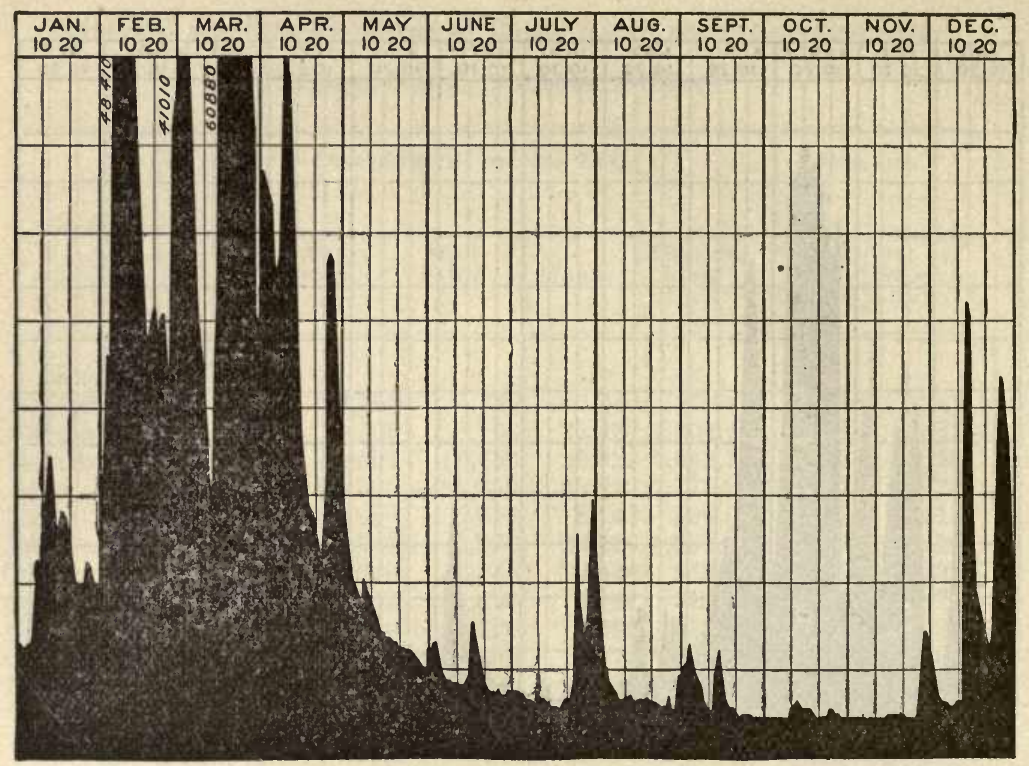

Fig. 11.-Discharge of Coosa River at Riverside, Ala., 1899.

Minimum monthly discharge of Coosa River at Riverside, Ala., with corresponding net horsepower per foot of fall on a water wheel realizing 80 per cent of theoretical power.

[Drainage area, 6,850 square miles.]

\begin{tabular}{|c|c|c|c|c|c|c|c|c|c|}
\hline & \multicolumn{3}{|c|}{1899} & \multicolumn{3}{|c|}{1900} & \multicolumn{3}{|c|}{1901} \\
\hline & 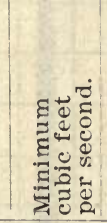 & 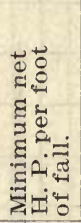 & 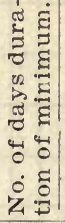 & 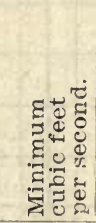 & 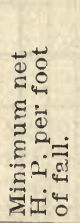 & 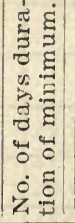 & 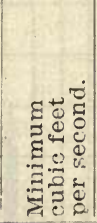 & 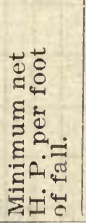 & 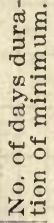 \\
\hline January & 6,390 & 581 & 2 & 4,285 & 390 & $\mid 2$ & 8,970 & 815 & 1 \\
\hline February & 17,330 & 1,575 & 1 & 5,700 & 518 & 1 & 8,970 & 815 & 1 \\
\hline March ... & 14,740 & 1,340 & 1 & 13,450 & 1,223 & 1 & 7,400 & 673 & 2 \\
\hline April .... & 11,410 & 1,037 & 1 & 11,350 & 1,032 & 1) & 14,500 & 1,310 & 2 \\
\hline May ..... & $4,900 \mid$ & 445 & 1 & 5,970 & 543 & 5 & 6,687 & 608 & 1 \\
\hline June ..... & 3,500 & 318 & 3 & 5,970 & 543 & 1 & 6,810 & 619 & 2 \\
\hline July ..... & 2,760 & 251 & 1 & 5,565 & 506 & 1 & 4,900 & 445 & 3 \\
\hline August ... & 2,600 & 236 & 1 & 4,050 & 369 & 1 & 4,400 & 400 & 1 \\
\hline September & 2,330 & 212 & 2 & 2,760 & 251 & 1 & 5,700 & 518 & 1 \\
\hline October .. & 2,330 & 212 & 11 & 3,100 & 282 & 1 & 4,285 & 390 & 1 \\
\hline November & 2,395 & 218 & 17 & 3,720 & 338 & 1 & 3,830 & 348 & 6 \\
\hline December & 2,920 & 265 & 2 & 5,835 & 530 & 2 & 4,050 & 368 & 1 \\
\hline
\end{tabular}

NoTE.-To find the minımum net horse power available at a shoal on this stream, near this station, for any month, multiply the tota? fall of the shoal by the "Net H. P. per foot of fall" in this table that month. 
2. COOSA RIVER AT ROME, GEORGIA.

Coosa River is formed by the junction of Etowah and Oostanaula rivers at Rome, Ga. The drainage area is 4,006 square miles. Both of the tributary rivers rise in the northern part of Georgia and flow for the most part through a hilly, broken country, well wooded, about one-fourth of the land being under cultivation. The Coosa River flows in a southwesterly direction into Alabama and joins the Tallapoosa 6 miles above Montgomery, Ala., to form Alabama River. Measurements of flow are made at Rome and at Riverside, I20 miles farther downstream. The measurements at Rome are made on the Oostanaula and Etowah just above their junction. Etowah River is measured at Second avenue bridge and the Oostanaula at Fifth avenue bridge in Rome, and the result added to give the flow of Coosa River. The gage height is taken from the United States Weather Bureau gage at Fifth avenue bridge, on the Oostanaula. There is practically no fall on Oostanaula River from Fifth avenue bridge to the junction, hence the gage is used as Coosa River gage and gives the fluctuations of Coosa River. This gage is a 4 by 6 inch timber, graduated to feet and tenths and fastened to the downstream left-hand corner of the first pier from the left bank. The zero of gage is 575.79 feet avove sea level. The United States Weather Bureau has maintained the station here for many years. It is now maintained only as a half-year station, from November I to April 3o, inclusive, but W. M. Towers, the river observer, kindly reads the gage and furnishes the Survey with monthly reports of the daily gage heights for the entire year without charge. Mr. Towers has kept the records for many years and has predicted floods with great precision. The channel of the Etowah is straight, current swift and unobstructed, but the Oostanaula is rather sluggish and somewhat obstructed by piers. The banks are high, but liable to overflow in times of high water.

The following discharge measurements were made during 1896-97-98 by Max Hall and others :

1896

September 29: Gage height, 0.20 feet; discharge, 1,209 second-feet. 1897 -

May 7: Gage height, 2.75 feet; discharge, 4,646 second-feet.

October 5: Gage height, 0.15 feet; discharge, 990 second-feet. 

feet.

1898

May 11: Gage height, 1.90 feet; discharge, 2,946 second-feet.

September 17: Gage height, 2.60 feet; discharge, 3,913 second-

October 11: Gage height, 5.05 feet; discharge, 8,324 second-feet.

October 22: Gage height, 4.10 feet; discharge, 6,489 second-feet. feet.

November 30: Gage height, 3.90 feet; discharge, 6,039 second-

Daily gage height, in feet, of Coosa River at Rome, Ga., for 189\%...

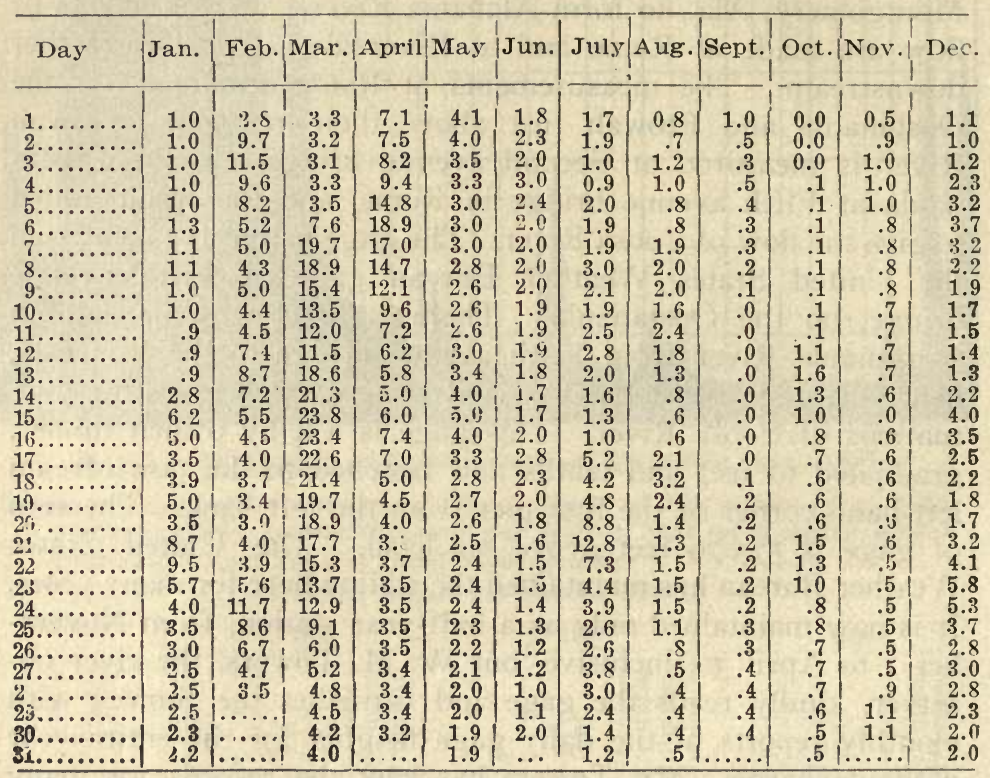


Daily gage height in feet, of Coosa River, at Rome, Ga., for 1898.

\begin{tabular}{|c|c|c|c|c|c|c|c|c|c|c|c|c|}
\hline Day & Jan. & Feb. & Mar. & April & May & Jun. & July & Aug. & Sept. & Oct. & Nov. & Dec. \\
\hline 1 & 8 & 3.6 & 1.2 & 9.0 & 2.8 & 1.4 & 1.2 & 4.8 & 2.0 & 2.0 & 2.2 & 4.2 \\
\hline & & 3.1 & 1. & 6.1 & 2.6 & 1.4 & 1.0 & $\begin{array}{l}4.0 \\
4.4\end{array}$ & 7.8 & 2.0 & 2.2 & 4.0 \\
\hline 3 & 1.7 & 2.9 & $1 . \%$ & 4.2 & & 1.4 & & 3.2 & 21.7 & 2.0 & 2.2 & 3.8 \\
\hline & 1.7 & 2.6 & 1.2 & 3.6 & 3 & 1.4 & 10 & 4.4 & 24.3 & 4.9 & 2.2 & 3.8 \\
\hline & 1.6 & 2.4 & 1.2 & $\begin{array}{l}0.0 \\
9.9\end{array}$ & 2 & 1.3 & 1.0 & 8.0 & 22.2 & 22.0 & .0 & 4.3 \\
\hline & 1.6 & $\tilde{2} .2$ & 1.2 & 17.2 & 2.1 & 1.3 & 1.3 & 5.6 & 20.0 & 23.8 & 2.2 & 5.0 \\
\hline & 1.3 & 2.0 & 1.2 & 14.5 & 2.0 & 1.3 & 2.0 & 4.4 & 17.6 & 19.0 & 2.6 & 4.3 \\
\hline & 1.3 & 1.8 & 1.2 & $\begin{array}{l}10.9 \\
10.9\end{array}$ & 2.0 & 1.3 & 2.8 & $\begin{array}{l}7.7 \\
4.4\end{array}$ & 16.4 & 18.4 & 2.4 & 4.0 \\
\hline & 1.3 & 1.8 & 1.2 & 7.0 & 2.0 & 1.3 & 3.2 & $\begin{array}{l}3.4 \\
3.4\end{array}$ & $\begin{array}{r}0.4 \\
9.7\end{array}$ & $\begin{array}{l}16.6 \\
\end{array}$ & 2.3 & $\begin{array}{l}4.0 \\
3.7\end{array}$ \\
\hline & 1.3 & 1.6 & 1.2 & 4.1 & 2.0 & 1.3 & & .0 & 5.0 & $\begin{array}{l}14.0 \\
14.0\end{array}$ & & $\begin{array}{l}3.4 \\
3.4\end{array}$ \\
\hline & 1.4 & & 1. & 4. & 2.0 & 1.3 & & & & & & 3.3 \\
\hline & $\therefore$ & & 1. & 3. & & 1.2 & & & & & & 3.3 \\
\hline & 1. 0 & & 1.2 & 3. & & 1.4 & & & & & & $\begin{array}{l}3.0 \\
3.2\end{array}$ \\
\hline & 4. & & 1. & & & 1.8 & & & 3. & & & 3.4 \\
\hline & 3. & & 1.6 & 3. & & 1.8 & & & & & & 3.4 \\
\hline & 3. & & 3.7 & & & 1.7 & & & 2. & & & 2.8 \\
\hline & 3. & & 7.3 & 3.0 & & 1. & & & 2. & & & 2.7 \\
\hline & & & 5.8 & 3.0 & 1. & & & & 2. & & & 2.6 \\
\hline & 2. & 1. & 3 . & 3. & 1. & 2. & & & 2. & & & \\
\hline & 4. & & 3. & 3. & 1. & & & & & & & \\
\hline & 6.5 & 1.2 & 2. & 3. & 1. & 3. & & & & & & 2.8 \\
\hline & 6. & & 2.5 & 3. & 1. & 3. & & & & & & 0 \\
\hline & & & 2. & 3. & 1. & & & & & & & \\
\hline & & & 2. & 7. & 1. & & & & & & & 3. \\
\hline & 0 & & 2.1 & 8.2 & 1.4 & 2. & & & & & & 3. \\
\hline & 14 & 1. & 2. & 6. & 1.4 & 1.8 & & & 3.0 & 3 & & 2. \\
\hline & & & 1.9 & 4. & 1. & 1 & & 4. & & & & \\
\hline & 11.6 & 1.2 & 1.8 & 4. & 1.4 & 1.8 & & 4.4 & & & & 2 \\
\hline & 8.6 & & 2. & & 1.4 & & 4. & 3.4 & & 2. & & \\
\hline & & …‥ & 8.5 & 3.2 & & 1.4 & & & 2.1 & 2.6 & 3.9 & 2. \\
\hline & 3.9 & . & 11.4 & ... & 1.4 & & 4. & 2.3 & .. & 2.4 & .... & \\
\hline
\end{tabular}


Rating table for Coosa River at Rome, Ga., for 1897 and 1898.

\begin{tabular}{|c|c|c|c|c|c|c|c|}
\hline 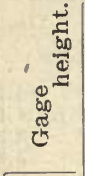 & 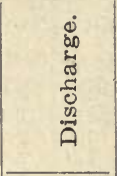 & 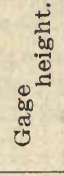 & 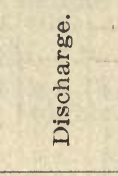 & 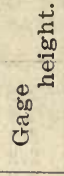 & 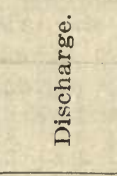 & 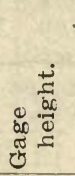 & 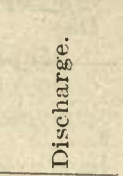 \\
\hline Feet. & Second $f t$. & Feet. & Second $f t$. & Feet. & Second $f t$. & Feet. & Second ft. \\
\hline-0.15 & 990 & 2.5 & 3,760 & 5.1 & 8,445 & 7.7 & 13,515 \\
\hline 0.0 & 1,070 & 2.6 & 3,910 & 5.2 & 8,640 & 7.8 & 13,710 \\
\hline 0.1 & 1,140 & 2.7 & 4,060 & 5.3 & 8,835 & 7.9 & 13,905 \\
\hline 0.2 & 1,210 & 2.8 & 4,220 & 5.4 & 9,030 & 8.0 & 14,100 \\
\hline 0.3 & 1,280 & 2.9 & 4,380 & 5.5 & 9,225 & 8.1 & 14,295 \\
\hline 0.4 & 1,360 & 3.0 & 4,540 & 5.6 & 9,420 & 8.2 & 14,490 \\
\hline 0.5 & 1,440 & 3.1 & 4,700 & 5.7 & 9,615 & 8.3 & 14,685 \\
\hline 0.6 & 1,520 & 3.2 & 4,860 & 5.8 & 9,810 & 8.4 & 14,880 \\
\hline 0.7 & 1,610 & 3.3 & 5,020 & 5.9 & 10,005 & 8.5 & 15,075 \\
\hline 0.8 & 1,700 & 3.4 & 5,180 & 6.0 & 10,200 & 8.6 & 15,270 \\
\hline 0.9 & 1,800 & 3.5 & 5,340 & 6.1 & 10,395 & 8.7 & 15,465 \\
\hline 1.0 & 1,900 & 3.6 & 5,520 & 6.2 & 10,590 & 8.8 & 15,660 \\
\hline 1.1 & 2,000 & 3.7 & 5,715 & 6.3 & 10,785 & 8.9 & 15,855 \\
\hline 1.2 & 2,110 & 3.8 & 5,910 & 6.4 & 10,980 & 9.0 & 16,050 \\
\hline 1.3 & 2,220 & 3.9 & 6,105 & 6.5 & 11,175 & 10.0 & 18,000 \\
\hline 1.4 & 2,330 & 4.0 & 6,300 & 6.6 & 11,370 & 11.0 & 19,950 \\
\hline 1.5 & 2,450 & 4.1 & 6,495 & 6.7 & 11,565 & 12.0 & 21,900 \\
\hline 1.6 & 2,570 & 4.2 & 6,690 & 6.8 & 11,760 & 13.0 & 23,850 \\
\hline 1.7 & 2,690 & 4.3 & 6,885 & 6.9 & 11,955 & 14.0 & 25,800 \\
\hline 1.8 & 2,810 & 4.4 & 7,080 & 7.0 & 12,150 & 15.0 & 27,750 \\
\hline 1.9 & 2,930 & 4.5 & 7,275 & 7.1 & 12,345 & 16.0 & 29,700 \\
\hline 2.0 & 3,060 & 4.6 & 7,470 & 7.2 & 12,540 & 17.0 & 31,650 \\
\hline 2.1 & 3,190 & 4.7 & 7,665 & 7.3 & 12,735 & 18.0 & 33,600 \\
\hline 2.2 & 3,320 & 4.8 & 7,860 & 7.4 & 12,930 & 20.0 & 37,500 \\
\hline 2.3 & 3,460 & 4.9 & 8,055 & 7.5 & 13,125 & 22.0 & 41,400 \\
\hline 2.4 & 3,610 & 5.0 & 8,250 & 7.6 & 13,320 & 24.0 & 45,300 \\
\hline
\end{tabular}

Note.-This taoie applied to the foregoing "Daily gage heights" gives the cubic feet per second flowing in the river on each date for which the gage height is given.

The following discharge measurements were made during I899 by Max Hall and others :

January 25-Gage height, 3.80 feet; discharge, 6,540 second-feet. January 25-Gage height, 3.60 feet; discharge, 5,932 second-feet. May 19-Gage height, 2.75 feet; discharge, 4,394 second-feet.

June 16-Gage height, 2.40 feet; discharge, 3,352 second-feet. August 4-Gage height, 1.45 feet; discharge, 2,835 second-feet. October 13-Gage height, 0.60 foot; discharge, 1,769 second-feet. 
Daily gage height, in feet, of Coosa River, at Rome, Georgia, for 1899.

\begin{tabular}{|c|c|c|c|c|c|c|c|c|c|c|c|c|}
\hline Day & an. & reb. & Iar. & April & May & Jun. & July & ug. & Sept. & Oct. & Nov. & Dec \\
\hline & $\begin{array}{l}3.60 \\
3.40 \\
3.00 \\
2.70 \\
2.60 \\
2.60 \\
3.60 \\
5.90 \\
5.90 \\
4.90 \\
4.00 \\
4.50 \\
4.00 \\
3.80 \\
3.60 \\
3.60 \\
4.00 \\
4.20 \\
4.00 \\
3.70 \\
3.30 \\
3.20 \\
3.100 \\
3.50 \\
3.80 \\
3.80 \\
3.30 \\
3.00 \\
3.00 \\
2.90 \\
4.40 \mid\end{array}$ & $\begin{array}{r}6.90 \\
7.80 \\
6.00 \\
9.20 \\
15.30 \\
18.20 \\
27.80 \\
24.00 \\
22.40 \\
21.00 \\
19.00 \\
16.50 \\
7.00 \\
5.00 \\
5.00 \\
5.50 \\
8.90 \\
9.50 \\
8.50 \\
7.70 \\
6.80 \\
6.90 \\
7.30 \\
6.60 \\
5.80 \\
5.50 \\
19.10 \\
23.40 \\
\ldots \ldots . . \\
\ldots \ldots . . \\
\ldots . . .\end{array}$ & \begin{tabular}{|r|}
19.70 \\
15.00 \\
8.60 \\
6.60 \\
7.80 \\
9.00 \\
8.00 \\
6.80 \\
5.70 \\
5.40 \\
5.20 \\
4.90 \\
4.50 \\
6.00 \\
16.60 \\
27.70 \\
29.20 \\
25.80 \\
24.90 \\
26.20 \\
24.60 \\
23.00 \\
22.60 \\
21.90 \\
18.00 \\
10.50 \\
7.70 \\
6.80 \\
8.80 \\
10.30 \\
10.20
\end{tabular} & $\begin{array}{r}13.20 \\
10.60 \\
7.90 \\
7.20 \\
9.50 \\
8.20 \\
8.20 \\
15.00 \\
13.40 \\
11.20 \\
9.50 \\
7.00 \\
6.40 \\
5.90 \\
5.60 \\
5.40 \\
5.20 \\
4.80 \\
4.70 \\
4.60 \\
4.30 \\
4.10 \\
4.00 \\
5.40 \\
7.40 \\
9.10 \\
6.70 \\
5.50 \\
4.80 \\
4.20\end{array}$ & $\begin{array}{l}4.00 \\
3.70 \\
3.70 \\
3.50 \\
3.50 \\
3.50 \\
3.70 \\
3.70 \\
3.60 \\
3.50 \\
3.30 \\
3.10 \\
3.10 \\
3.00 \\
3.00 \mid \\
2.90 \mid \\
2.8 \mathrm{u} \\
2.80 \mid \\
2.80 \mid \\
2.80 \\
2.60 \\
2.60 \mid \\
2.40 \\
2.60 \mid \\
2.50 \mid \\
2.40 \mid \\
2.20 \\
2.20 \\
2.00 \\
2.00 \\
3.30\end{array}$ & $\begin{array}{l}3.00 \\
2.60 \\
2.60 \\
2.00 \\
2.00 \\
2.00 \\
2.00 \\
1.90 \\
1.80 \\
1.80 \\
1.80 \\
2.20 \\
3.80 \\
4.01 \\
3.50 \\
2.50 \\
2.10 \\
2.00 \\
2.00 \\
2.00 \\
1.80 \\
2.20 \\
1.70 \\
1.70 \\
1.70 \\
1.70 \\
2.10 \\
1.90 \\
1.90 \\
1.80\end{array}$ & \begin{tabular}{|r|}
1.70 \\
1.50 \\
1.00 \\
.90 \\
2.00 \\
1.90 \\
1.90 \\
3.00 \\
2.10 \\
1.90 \\
2.50 \\
2.80 \\
2.00 \\
1.60 \\
1.30 \\
1.80 \\
5.20 \\
4.20 \\
4.80 \\
8.80 \\
12.80 \\
7.90 \\
4.80 \\
3.90 \\
2.60 \\
2.60 \\
3.80 \\
3.00 \\
2.40 \\
1.40 \\
1.20
\end{tabular} & $\begin{array}{r}2.20 \\
1.90 \\
1.70 \\
1.50 \\
1.40 \\
1.50 \\
1.60 \\
1.50 \\
1.80 \\
1.60 \\
1.40 \\
1.40 \\
1.20 \\
1.10 \\
1.30 \\
1.90 \\
1.60 \\
1.40 \\
1.10 \\
.90 \\
.90 \\
.80 \\
.80 \\
.70 \\
.70 \\
.70 \\
2.50 \\
2.50 \\
2.50 \\
2.00\end{array}$ & $\begin{array}{l}3.40 \\
2.00 \\
1.60 \\
1.40 \\
1.30 \\
1.30 \\
1.20 \\
1.00 \\
1.00 \\
1.00 \\
2.90 \\
2.30 \\
1.50 \\
1.00 \\
.90 \\
.80 \\
.60 \\
.60 \\
.60 \\
.70 \\
.60 \\
.60 \\
.50 \\
.50 \\
.50 \\
.50 \\
.60 \\
.50 \\
.40\end{array}$ & $\begin{array}{r}0.40 \\
.30 \\
.30 \\
.30 \\
.30 \\
.50 \\
.70 \\
.70 \\
.80 \\
.60 \\
1.00 \\
.90 \\
.70 \\
.70 \\
.60 \\
.60 \\
.60 \\
.60 \\
.70 \\
.70 \\
.60 \\
.50 \\
.40 \\
.40 \\
.40 \\
.40 \\
.40 \\
.50\end{array}$ & $\begin{array}{r}0.7 \\
.6 ! \\
.5 \\
.5 \\
.4 \\
.4 \\
.3 \\
.3 \\
.3 \\
.3 \\
.3 \\
.3 \\
.3 \\
.4 \\
.4 \\
.5 \\
.9 \\
.7 \\
.5 \\
.5 \\
.5 \\
.4 \\
1.0 \\
2.1 \\
1.5 \\
2.5 \\
3.0 \\
2.2 \\
1.9 \\
1.4\end{array}$ & $\begin{array}{l}1.1 \\
1.1 \\
1.5 \\
1.3 \\
1.1 \\
1.0 \\
.9 \\
.8 \\
.8 \\
.8 \\
.8 \\
2.8 \\
6.1 \\
5.0 \\
3.2 \\
.0 \\
1.8 \\
1.7 \\
1.3 \\
1.6 \\
2.0 \\
.0 \\
1.8 \\
7.2 \\
7.5 \\
5.0 \\
3.5 \\
3.0 \\
3.0 \\
3.4 \\
2.0\end{array}$ \\
\hline
\end{tabular}


Rating table for Coosa River at Rome. Georgia, for 1899.

\begin{tabular}{|c|c|c|c|c|c|c|c|}
\hline 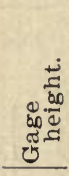 & 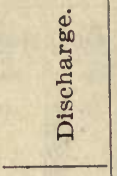 & 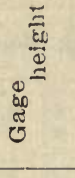 & 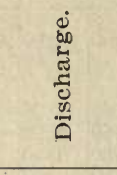 & 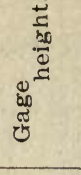 & 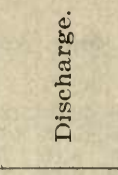 & 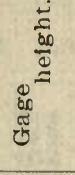 & 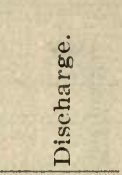 \\
\hline Feet. & Second ft. & Feet. & Second $f t$ & Feet. & Second $f t$. & Feet. & secund ft. \\
\hline 1. 0 & 2,030 & 8.1 & 14,941 & 15.9 & 30,619 & 23.0 & $4\{, 890$ \\
\hline 1.1 & 2,124 & 8.2 & 15,142 & 16.0 & 30,820 & 23.1 & 45,091 \\
\hline 1.2 & 2,218 & 9.0 & 16,750 & 16.1 & 31,021 & 23.2 & 45,292 \\
\hline 1.3 & 2,312 & 9.1 & 16,951 & 16.2 & 31,222 & 23.3 & 45,493 \\
\hline 1.4 & 2,406 & 9.2 & 17,152 & 16.3 & 31,423 & 23.4 & 45,694 \\
\hline 1.5 & 2,500 & 9.3 & 17,353 & 16.4 & 31,624 & 23.5 & 45,895 \\
\hline 1.6 & 2,620 & 9.4 & 17,554 & 16.5 & 31,825 & 23.6 & 46,096 \\
\hline 1.7 & 2,740 & 9.5 & 17,755 & 16.6 & 32,026 & 23.7 & 46,297 \\
\hline 1.8 & 2,860 & 9.6 & 17,956 & 16.7 & 32,227 & 23.8 & 46,498 \\
\hline 1.9 & 2,980 & 9.7 & 18,157 & 16.8 & 32,428 & 23.9 & 46,699 \\
\hline 2.0 & 3,100 & 9.8 & 18,358 & 16.9 & 32,629 & 24.0 & 46,900 \\
\hline 2.1 & 3,260 & 9.9 & 18,559 & 17.0 & 32,830 & 24.1 & 47,101 \\
\hline 2.2 & 3,420 & 10.0 & 18,760 & 17.1 & 33,031 & 24.2 & 47,302 \\
\hline 2.3 & 3,580 & 10.1 & 18,961 & 17.2 & 33,232 & 24.3 & 47,503 \\
\hline$\ddot{2} .4$ & 3,740 & 10.2 & 19,162 & 17.3 & 33,433 & 24.4 & 47,704 \\
\hline 2.5 & 3,900 & 10.3 & 19,363 & 17.4 & 33,634 & 24.5 & 47,905 \\
\hline 2.6 & 4,060 & 10.4 & 19,564 & 17.5 & 33,835 & 24.6 & 48,106 \\
\hline 2.7 & 4,220 & 10.5 & 19,765 & 17.6 & 34,036 & 24.7 & 48,307 \\
\hline $2 . \dot{s}$ & 4,380 & 10.6 & 19,966 & 17.7 & 34,237 & 24.8 & 48,508 \\
\hline 2.9 & 4,540 & 10.7 & 20,167 & 17.8 & 34,438 & 24.9 & 48,709 \\
\hline 3.0 & 4,700 & 10.8 & 20,368 & 17.9 & 34,639 & 25.0 & 48,910 \\
\hline 3.1 & 4,900 & 10.9 & 20,569 & 18.0 & 34,840 & 25.1 & 49,111 \\
\hline 3.2 & 5,100 & 11.0 & 20,770 & 18.1 & 35,041 & 25.2 & 49,312 \\
\hline 3.3 & 5,300 & 11.1 & 20,971 & 18.2 & 35,242 & 25.3 & 49,513 \\
\hline 3.4 & 5,500 & 11.2 & 21,172 & 18.3 & 35,443 & 25.4 & 49,714 \\
\hline 3.5 & 5,700 & 11.3 & 21,373 & 18.4 & 35,644 & 25.5 & 49,915 \\
\hline 3.6 & 5,900 & 11.4 & 21,574 & 18.5 & 35,845 & 25.6 & 50,116 \\
\hline 3.7 & 6,100 & 11.5 & 21,775 & 18.6 & 36,046 & 25.7 & 50,317 \\
\hline 3.8 & 6,300 & 11.6 & 21,976 & 18.7 & 36,247 & 25.8 & 50,518 \\
\hline 3.9 & 6,500 & 11.7 & 22,177 & 18.8 & 36,448 & 25.9 & 50,719 \\
\hline 4.0 & 6,700 & 11.8 & 22,378 & 18.9 & 36,649 & 26.0 & 50,920 \\
\hline 4.1 & 6,901 & 11.9 & 22,579 & 19.0 & 36,850 & 26.1 & 51,121 \\
\hline 4.2 & 7,102 & 12.0 & 22,780 & 19.1 & 37,051 & 26.2 & 51,322 \\
\hline 4.3 & 7,303 & 12.1 & 22,981 & 19.2 & 37,252 & 26.3 & 51,523 \\
\hline 4.4 & 7,504 & 12.2 & 23,182 & 19.3 & 37,453 & 26.4 & 51,724 \\
\hline 4.5 & 7,705 & 12.3 & 23,383 & 19.4 & 37,654 & 26.5 & 51,925 \\
\hline 4.6 & 7,906 & 12.4 & 23,584 & 19.5 & 37,855 & 26.6 & 52,126 \\
\hline 4.7 & 8,107 & 12.5 & 23,785 & 19.6 & 38,036 & 26.7 & 52,327 \\
\hline 4.8 & 8,308 & 12.6 & 23,986 & 19.7 & 38,257 & 26.8 & 52,528 \\
\hline 4.9 & 8,509 & 12.7 & 24,187 & 19.8 & 38,458 & 26.9 & 52,729 \\
\hline 5.0 & 8,710 & 12.8 & 24,388 & 19.9 & 38,659 & 27.0 & 52,930 \\
\hline 5.1 & 8,911 & 12.9 & 24,589 & 20.0 & 38,860 & 27.1 & 53,131 \\
\hline 5.2 & 9,112 & 13.0 & 24,790 & 20.1 & 39,061 & 27.2 & 53,332 \\
\hline 5.3 & 9,313 & 13.1 & 24,991 & 20.2 & 39,262 & 27.3 & 53,533 \\
\hline 5.4 & 9,514 & 13.2 & 25,192 & 20.3 & 39,463 & 27.4 & 53,734 \\
\hline
\end{tabular}


Rating table for Coosa River at Rome, Ga., for 18.99.

\begin{tabular}{|c|c|c|c|c|c|c|c|}
\hline . & 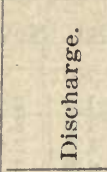 & 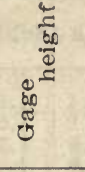 & 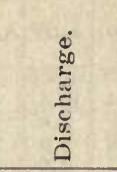 & 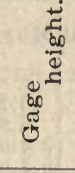 & 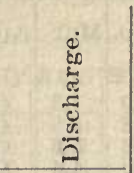 & 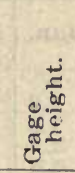 & 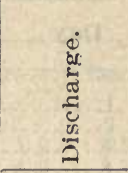 \\
\hline Feet. & Second $f t$. & Feet. & Seeond $f t$. & & Second $f t$. & Feet. & second $f t$. \\
\hline 5.5 & 9,715 & 13.3 & 25,393 & 20.4 & 39,664 & 27.5 & 53,935 \\
\hline 5.6 & 9,916 & 13.4 & 25,594 & 20.5 & 39,865 & 27.6 & 54,136 \\
\hline 5.7 & 10,117 & 13.5 & 25,795 & 20.6 & - 40,066 & 27.7 & 54,337 \\
\hline 5.8 & 10,318 & 13.6 & 5,996 & 20.7 & 40,267 & 27.8 & 54,538 \\
\hline 5.9 & 10,519 & 13.7 & 26,197 & 20.8 & 40,468 & 27.5 & 54,739 \\
\hline 6.0 & 10,720 & 13.8 & 26,398 & 20.9 & 40,669 & 28.0 & 54,940 \\
\hline 6.1 & 10,921 & 13.9 & 26,5 & 21.0 & 40,870 & 28.1 & 55,141 \\
\hline 6.2 & 11,122 & 14.0 & 26,800 & 21.1 & 41,071 & 28.2 & 55,342 \\
\hline 6.3 & 11,323 & 14.1 & 27,001 & 21.2 & 41,272 & 28.3 & 55,543 \\
\hline 6.4 & 11,524 & 14.2 & 27,202 & 21.3 & 41,473 & 28.4 & 55,744 \\
\hline 6.5 & 11,725 & 14.3 & $\angle 7,403$ & 21.4 & 41,674 & 28.5 & 55,945 \\
\hline 6.6 & 11,926 & 14.4 & 27,604 & 21.5 & 41,875 & 28.6 & 56,146 \\
\hline 6.7 & 12,127 & 14.5 & 27,805 & 21.6 & 42,076 & 28.7 & 56,347 \\
\hline 6.8 & 12,328 & 14.6 & 28,006 & 21.7 & 42,277 & 28.8 & 56,548 \\
\hline 6.9 & 12,529 & 14.7 & 28,207 & 21.8 & 42,478 & 28.9 & 56,749 \\
\hline 7.0 & 12,730 & 14.8 & 28,408 & 21.9 . & 42,679 & 29.0 & 56,950 \\
\hline 7.1 & 12,931 & 14.9 & 28,609 & 22.0 & 42,880 & 29.1 & 57,151 \\
\hline 7.2 & 13,132 & 15.0 & 28,810 & 22.1 & 43,081 & 29.2 & 57,352 \\
\hline 7.3 & 13,333 & 15.1 & 29,011 & 22.2 & 43,282 & 29.3 & 57,553 \\
\hline 7.4 & 13,534 & 15.2 & 29,212 & 22.3 & 43,483 & 29.4 & 57,754 \\
\hline 7.5 & 13,735 & 15.3 & 29,413 & 22.4 & 43,684 & 29.5 & 57,955 \\
\hline 7.6 & 13,936 & 15.4 & 29,614 & 22.5 & 43,885 & 29.6 & 58,156 \\
\hline 7.7 & 14,137 & 15.5 & 29,815 & 22.6 & 44,086 & 29.7 & 58,357 \\
\hline 7.8 & 14,338 & 15.6 & 30,016 & 22.7 & 44,287 & 29.8 & 58,558 \\
\hline 7.9 & 14,539 & 15.7 & 30,217 & 22.8 & 44,488 & 29.9 & 58,759 \\
\hline 8.0 & 14,740 & 15.8 & 30,418 & 22.9 & 44,689 & 30.0 & 58,960 \\
\hline
\end{tabular}

The following discharge measurements were made during I 900 by Max Hall and others :

Feb. 21-Gage height, 4.80 feet; discharge, 8,115 second-feet. May 19-Gage height, 2.30 feet; discharge, 4,496 second-feet. Sept. 13-Gage height, 0.90 foot; discharge, 1,992 second-feet. Dec. \&-Gage height, 3.73 feet; discharge, 6,066 second-feet. 
Daily goge height, in feet, of Coosa River, at Rome, Georgia, for 1900.

\begin{tabular}{|c|c|c|c|c|c|c|c|c|c|c|c|c|}
\hline Day & Jan. & Feb. & Mar. & April & May & Jun. & July & Aug. & Sept.| & Oet. & Nov.| & De \\
\hline & 2.0 & 2.0 & 4.2 & 4.4 & 6.2 & 2.4 & 10.5 & 3.4 & 1.5 & 1.2 & 2.1 & 3.2 \\
\hline & & & & & & & & & & & & \\
\hline & & & & & & 2. & 8. & & & & & \\
\hline & & & & & & 4. & 7. & & 1. & & & \\
\hline & 1. & & 4. & 4. & & 4. & 5. & & 1. & & & \\
\hline & & & & 4. & & 4. & 4. & & & & & \\
\hline & 1. & & 5. & 3. & 3. & 4. & 3. & & & & & \\
\hline & & & & 3. & & 13. & 3. & & & & & \\
\hline & & 4. & 15.0 & 3. & 3.0 & 12.6 & 4. & & 1. & & & \\
\hline & & & & & & 8. & 4. & & & & & \\
\hline & & 7. & 10.3 & & & 5.9 & & & & & & \\
\hline & & & & 11.0 & & 5. & 3. & & .8 & & & \\
\hline & 9. & 22.6 & 5. & 7.4 & & 5.2 & 3. & & .8 & & & 2. \\
\hline & & 2 & & 5. & & 5. & & & .8 & & & \\
\hline & 5. & & & 4. & & 4.2 & 3. & & 6.5 & & & \\
\hline & & 21.2 & 5. & 5. & 2. & 3.8 & 3. & & 11.1 & & & 2. \\
\hline & & 18.0 & 5. & & & 4.8 & 3. & & 7.0 & & & 2 . \\
\hline & & 10.7 & & 11. & 2. & 6. & 3. & & & & & 2. \\
\hline & & & & & & & & & 2. & & & 2. \\
\hline & 11 & & 15. & & & 7. & & & & & & 2. \\
\hline & & 4. & 17. & & & 4. & & & & & & 6. \\
\hline & & 6. & 14. & & & 3. & & & & & & 8. \\
\hline & 5. & 7.6 & 10.4 & 10. & & & 2. & & & & & 7. \\
\hline & & 6. & & 8. & 2. & 14. & 2. & & & & & 6. \\
\hline & & 5.8 & & & & & & & 1. & & & 6.6 \\
\hline & & 5.2 & 13.0 & 6. & & 17 & & & & & 11 & \\
\hline & & 4.6 & 12. & 5. & & & & 1. & 1.4 & & 11. & 4.0 \\
\hline & & 4.0 & & 4. & 2 . & & & 1. & 1. & 2. & 8. & 3.8 \\
\hline & & $\cdots$ & 5.8 & 4. & 2. & 14. & & 1. & 1. & 2. & & 3.6 \\
\hline & 2. & & 5.7 & 6.0 & 2. & 10.0 & 4. & 1. & 1.3 & 2. & 4.0 & 3. \\
\hline & 2.0 & & 5.3 & & 3.0 & & 4.0 & 1.5 & & 2.1 & $1 \ldots$ & 5. \\
\hline
\end{tabular}

The following discharge measurements were made during Igor by Max Hall and others:

Jan. 23-Gage height, 3.60 feet; discharge, 6,454 second-feet. April 5-Gage height, 9.90 feet; discharge, 16,692 second-feet. June 22-Gage height, 3.70 feet; discharge, 6,030 second-feet. Oct. 15-Gage height, 3.15 feet; discharge, 5,388 second-feet. 
Daily gage height of Coosa River at Rome, Georgia, for 1901.

\begin{tabular}{|c|c|c|c|c|c|c|c|c|c|c|c|c|}
\hline Day & Jan. & Feb. & Mar. & April & May & Jun. & July & Aug. & Sept. & Oct. & Nov. & - D \\
\hline & & 6.4 & 3.0 & 8.8 & 4.0 & 10.6 & 3.6 & 1.8 & 6.4 & 2.6 & 1.2 & \\
\hline & & & & & & & & & & & & \\
\hline & & & 3. & 13 & & 5 & & & & & & \\
\hline & & 15.8 & 3. & 13. & & 6. & & 1 & & & & \\
\hline & & 18. & 3. & 10 & & 5. & & $\overline{1}$ & & & & \\
\hline & & 13. & 3. & 7 & & 4. & & 2 & & & & \\
\hline & & & & & & 7. & & 5 & & & & \\
\hline & 3. & & 3. & 5. & 3. & 7. & & 5. & 2. & & & \\
\hline & & 9.6 & 2. & 5. & & 5. & & & & & & \\
\hline 10 & 2.8 & 12.5 & 5.5 & 4. & 3. & 4. & & 2. & 2 & & & \\
\hline & 8.8 & 10.5 & 7. & 4 & & 4. & & & 2. & 1 & & \\
\hline & 23.5 & 7. & 8. & 4. & 2 . & 3. & & & 2 & 1. & & \\
\hline & 27 & & 6. & 4 & 2.8 & 3. & & 3 & 1. & & & \\
\hline & 23 & 5. & 4. & 10 & 2. & 4. & & 2 & 2 & & & \\
\hline & 21 & & 4. & 10. & 2. & 4. & & 4. & 3. & & & 16 \\
\hline & 19.8 & 4.8 & 3. & & 2. & 6. & & & & 2 & & 17 \\
\hline 7. & 17. & 4.2 & 3. & 5 & 2. & 6. & 1. & 10. & 6 & 2 & & 14 \\
\hline & & 4. & 3. & & 2. & 5. & & & 11 & & & 11 \\
\hline & 5. & 4.2 & 3. & 9 & 2. & 4. & & 10. & 11. & 2 & & 13 \\
\hline & 4. & 4.0 & 3. & & 3 & 4. & & & & & & \\
\hline & 3.8 & 3.8 & 3. & 17. & 10. & 3. & 2.4 & & & 1. & & \\
\hline & & 3. & 3 & & & 3. & & & & & & ? \\
\hline & 3.8 & 3.6 & 3. & & 26. & 3. & & & 3 & 1. & 1 & 2 \\
\hline & & 3. & 3. & 12. & 21. & 3. & & & & 1. & & \\
\hline & 6.7 & 3.5 & 3. & 6. & 18.9 & 2.7 & 2. & & 2 & 1. & & \\
\hline & 6.6 & 3.2 & 22. & 5. & 16.5 & 2.7 & 1. & 13. & & & & \\
\hline & 5.4 & 3.2 & 27.0 & 4. & 11.1 & 3.2 & $1 . ?$ & 6. & & 1. & & \\
\hline & 5.2 & 3.0 & 24.5 & 4. & 5.5 & 3.0 & 2.8 & & 2. & 1. & 1. & 6. \\
\hline & 5.0 & & 21.3 & 4. & 4. & 3.6 & 1. & & 2. & 1.3 & 1.3 & 21. \\
\hline & 4.6 & & 19.2 & 4.1 & 4. & 3.6 & 1.8 & 6. & 2.5 & 1.3 & 1.3 & 29. \\
\hline & & & 16.1 & $\cdots$ & 5.4 & & 1.6 & 5.6 & & 1.2 & ..... & 32. \\
\hline
\end{tabular}

Rating table for Coosa River at Rome, Ga., for 1900 and 1901.

\begin{tabular}{|c|c|c|c|c|c|c|c|}
\hline 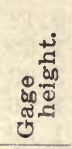 & 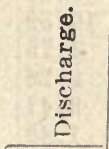 & 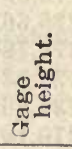 & $\begin{array}{l}\dot{0} \\
\dot{0} \\
\frac{\pi}{\pi} \\
0 \\
0 \\
0 \\
0 \\
0\end{array}$ & 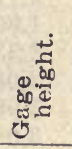 & 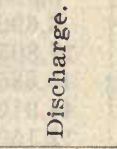 & & 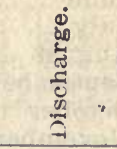 \\
\hline Feet. & Second $f t$. & Feet. & Second $f l$. & Feet. & Second $f t$. & Feet & second ft. \\
\hline 0.8 & 1,930 & 1.6 & 2,850 & 2.4 & 4,000 & 3.2 & 5,230 \\
\hline 0.9 & 2,020 & 1.7 & 2,985 & 2.5 & 4,150 & 3.3 & 5,405 \\
\hline 1.0 & 2,110 & 1.8 & 3,120 & 2.6 & 4,300 & 3.4 & 5,580 \\
\hline 1.1 & 2,230 & 1.9 & 3,260 & 2.7 & 4,450 & 3.5 & 5,755 \\
\hline 1.2 & 2,350 & 2.0 & 3,400 & 2.8 & 4,600 & $* 3.6$ & 5,930 \\
\hline 1.3 & 2,475 & 2.1 & 3,550 & 2.9 & 4,750 & & \\
\hline 1.4 & 2,600 & 2.2 & 3,700 & 3.0 & 4,900 & & \\
\hline 1.5 & 2,725 & 2.3 & 3,850 & 3.1 & 5,065 & & \\
\hline
\end{tabular}

*Above $3.6 \mathrm{ft}$. gage height, the rating for $1900-1901$ is the same as for 1899. 
Estimated monthly discharge of Coosa River at Rome, Ga. [Drainage area, 4,006 square miles.]

\begin{tabular}{|c|c|c|c|c|c|c|}
\hline \multirow[b]{2}{*}{ Month. } & \multicolumn{3}{|c|}{$\begin{array}{l}\text { Discharge in second- } \\
\text { feet. }\end{array}$} & \multirow[b]{2}{*}{$\begin{array}{l}\text { Total in } \\
\text { acre-ft. }\end{array}$} & \multicolumn{2}{|c|}{ Run-off. } \\
\hline & 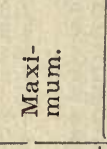 & 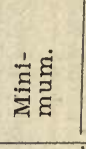 & 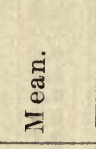 & & 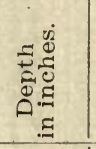 & 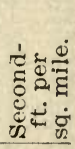 \\
\hline 1897. & & & & & & \\
\hline January $\ldots . . .$. & 17,025 & $\begin{array}{l}1,800 \\
4220\end{array}$ & 4,820 & & 1.38 & 1.20 \\
\hline & $\begin{array}{l}20,925 \\
44,910\end{array}$ & $\begin{array}{l}4,220 \\
4,700\end{array}$ & $\begin{array}{l}10,100 \\
22,537\end{array}$ & $\begin{array}{r}560,926 \\
1,385,755\end{array}$ & $\begin{array}{l}2.62 \\
6.49\end{array}$ & $\begin{array}{l}2.52 \\
5.63\end{array}$ \\
\hline $\begin{array}{l}\text { March } \ldots \ldots \\
\text { April } \ldots \ldots\end{array}$ & 35,150 & 4,860 & 12,304 & $\begin{array}{r}1,000,100 \\
732,137\end{array}$ & 3.43 & $\begin{array}{l}3.00 \\
3.07\end{array}$ \\
\hline May ................. & 8,250 & 2,930 & 4,421 & 271,838 & 1.27 & 1.10 \\
\hline June .......... & 4,540 & 1,900 & 2,884 & 171,610 & 0.80 & 0.72 \\
\hline July $\ldots \ldots \ldots \ldots$ & 23,460 & 1,800 & 5,184 & 318,754 & 1.50 & 1.30 \\
\hline August $\ldots \ldots \ldots$ & 4,860 & 1,360 & 2,256 & 138,717 & 0.64 & 0.56 \\
\hline September ..... & 1,900 & 900 & 1,106 & 65,811 & 0.31 & 0.28 \\
\hline October .......... & 2,570 & 1,010 & 1,518 & 93,339 & 0.44 & 0.38 \\
\hline November $\ldots \ldots \ldots$ & 2,000 & 1,440 & 1,626 & 96,754 & 0.46 & 0.41 \\
\hline December $\quad . . . \ldots$ & 9,810 & 1,900 & 4,086 & 251,240 & 1.18 & 1.02 \\
\hline 1898. & & & & & & \\
\hline January ....... & 26,970 & 2,220 & 7,272 & 447,138 & 2.10 & 1.82 \\
\hline February .... & 5,520 & 2,110 & 2,705 & 150,228 & 0.71 & 0.68 \\
\hline March $\ldots \ldots \ldots \ldots$ & 20,730 & 2,110 & 4,384 & 269,563 & 1.27 & 1.10 \\
\hline April .......... & 32,040 & 4,540 & 9,430 & 561,123 & 2.63 & 2.36 \\
\hline May $\ldots \ldots \ldots$ & 4,220 & 2,330 & 2,778 & 170,814 & 0.79 & 0.69 \\
\hline June ...... & 5,520 & 2,110 & 2,866 & 170,538 & 0.80 & 0.72 \\
\hline July $\ldots \ldots$ & 6,69 ㅇ & 1,900 & 3,670 & 225,661 & 10.59 & 9.17 \\
\hline August .... . . & 17,805 & 2,930 & 6,079 & 373,786 & 1.75 & 1.52 \\
\hline September .... & 45,885 & 3,060 & 12,114 & 720,832 & 2.26 & 3.03 \\
\hline October ..... & 44,910 & 3,060 & 11,830 & 727,403 & 3.41 & 2.96 \\
\hline November .... & 12,150 & 3,060 & 5,213 & 310,194 & 1.45 & 1.30 \\
\hline December $\quad \ldots \ldots \ldots$ & 8,250 & 3,610 & 4,996 & 307,194 & 1.44 & 1.25 \\
\hline 1899. & & & & & & \\
\hline January . & 10,519 & 4,060 & 6,092 & 374,582 & 1.75 & 1.52 \\
\hline February . & 54,538 & 8,710 & 22,536 & $1,251,586$ & 5.85 & 5.62 \\
\hline March .... & 57,352 & 7,705 & 26,314 & $1,617,985$ & 7.57 & 6.57 \\
\hline April $\ldots \ldots \ldots \ldots \ldots$ & 28,810 & 6,700 & 13,333 & 793,369 & 3.72 & 3.33 \\
\hline May $\ldots \ldots \ldots \ldots$ & 6,700 & 3,100 & 4,783 & 294,095 & 1.37 & 1.19 \\
\hline June $\ldots . . . .$. & 6,700 & 2,740 & 3,489 & 207,610 & 0.97 & 0.87 \\
\hline July $\ldots .$. & 24,388 & 1,950 & 5,499 & 338,120 & 1.58 & 1.37 \\
\hline August $\ldots$ & 3,900 & 1,790 & 2,595 & 159,560 & 0.75 & 0.65 \\
\hline September .. & 5,500 & 1,550 & 2,219 & 132,040 & $0.61 \mid$ & 0.55 \\
\hline October ..... & 2,030 & $1,470^{\top}$ & 1,684 & 103,545 & 0.48 & 0.42 \\
\hline November ..... & 4,700 & 1,470 & 2,009 & 119,544 & 0.56 & 0.50 \\
\hline December....$\ldots$. & 13,735 & 1,870 & 4,314 & $265,258 i$ & 1.25 & 1.08 \\
\hline
\end{tabular}


Fstimated monthly discharge of Coosa River at Rome, Georgia.

[Drainage area, 4,006 square miles.]

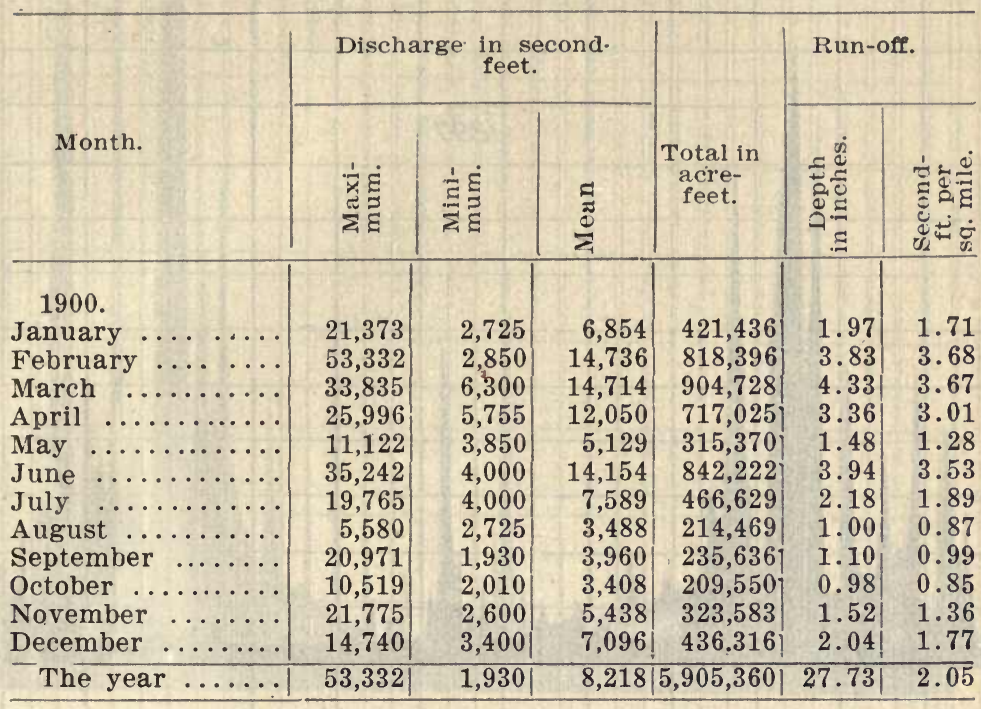

Estimated monthly discharge of Coosa River at Rome, Ga.

[Drainage area, 4,006 square miles.]

\begin{tabular}{|c|c|c|c|c|c|}
\hline \multirow[b]{2}{*}{ Month. } & \multicolumn{3}{|c|}{ Discharge in second-feet. } & \multicolumn{2}{|c|}{ Run-off. } \\
\hline & $\begin{array}{l}\text { Maxi- } \\
\text { mum. }\end{array}$ & $\begin{array}{l}\text { Mini- } \\
\text { mum. }\end{array}$ & Mean. & $\begin{array}{c}\text { Depth } \\
\text { in } \\
\text { inches. }\end{array}$ & $\begin{array}{l}\text { Second- } \\
\text { feet per } \\
\text { square } \\
\text { mile. }\end{array}$ \\
\hline 1901. & & & & & \\
\hline $\begin{array}{l}\text { January } \\
\text { February }\end{array}$ & $\begin{array}{l}52,930 \\
35,845\end{array}$ & $\begin{array}{l}4,600 \\
4,900\end{array}$ & $\begin{array}{l}15,450 \\
12,186\end{array}$ & $\begin{array}{l}4.45 \\
3.17\end{array}$ & $\begin{array}{l}3.86 \\
3.04\end{array}$ \\
\hline March... & 52,930 & 4,600 & 13,406 & 3.85 & 3.34 \\
\hline April $\ldots \ldots \ldots \ldots \ldots \ldots$ & 36,046 & 6,901 & 15,578 & 4.33 & 3.88 \\
\hline May $\ldots \ldots \ldots \ldots \ldots \ldots$ & 51,724 & 4,150 & 12,533 & 3.60 & 3.12 \\
\hline June & 19,966 & 4,450 & 8,316 & 2.32 & 2.08 \\
\hline July $\ldots \ldots \ldots \ldots \ldots$ & 9,715 & 2,850 & 4,441 & 1.27 & 1.10 \\
\hline August $\ldots \ldots \ldots \ldots \ldots$ & 45,292 & 2,850 & 13,780 & 3.97 & 3.44 \\
\hline September $\ldots \ldots \ldots$. . . . . . & 21,172 & 3,120 & 6,389 & 1.77 & 1.59 \\
\hline October $\ldots \ldots \ldots \ldots \ldots$ & 5,230 & 2,350 & 3,414 & .98 & .85 \\
\hline November $\ldots \ldots \ldots \ldots \ldots$ & 2,850 & 2,110 & 2,316 & .65 & .58 \\
\hline December $\ldots \ldots \ldots \ldots \ldots$ & 64,186 & 2,475 & 13,428 & 3.86 & 3.35 \\
\hline The year $\ldots \ldots \ldots \ldots \ldots$ & 64,186 & 2,110 & 10,103 & 34.22 & 2.52 \\
\hline
\end{tabular}



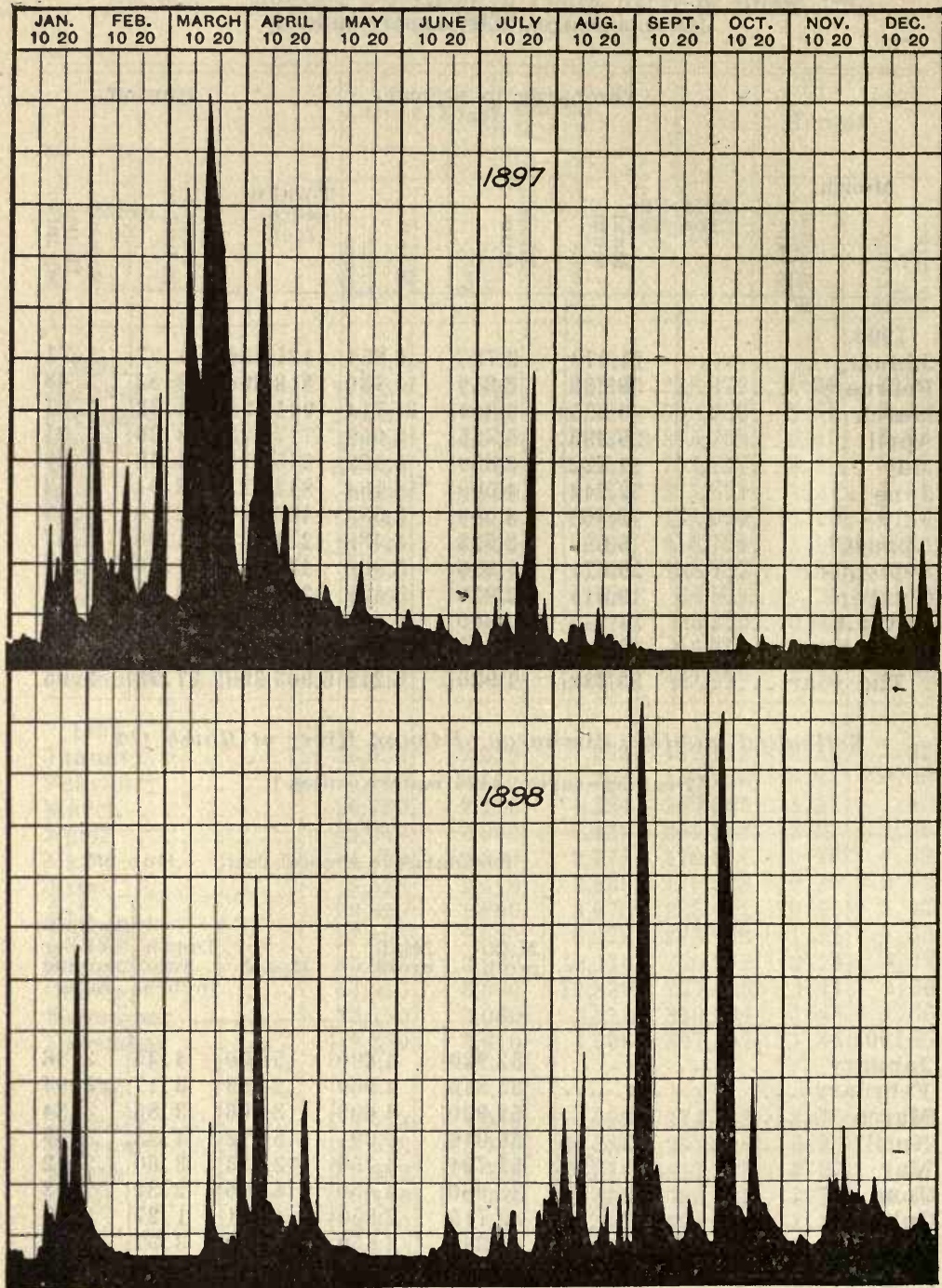

Fig. 12-Discharge of Coosa River at Rome, Ga., 1897 and 1898. 


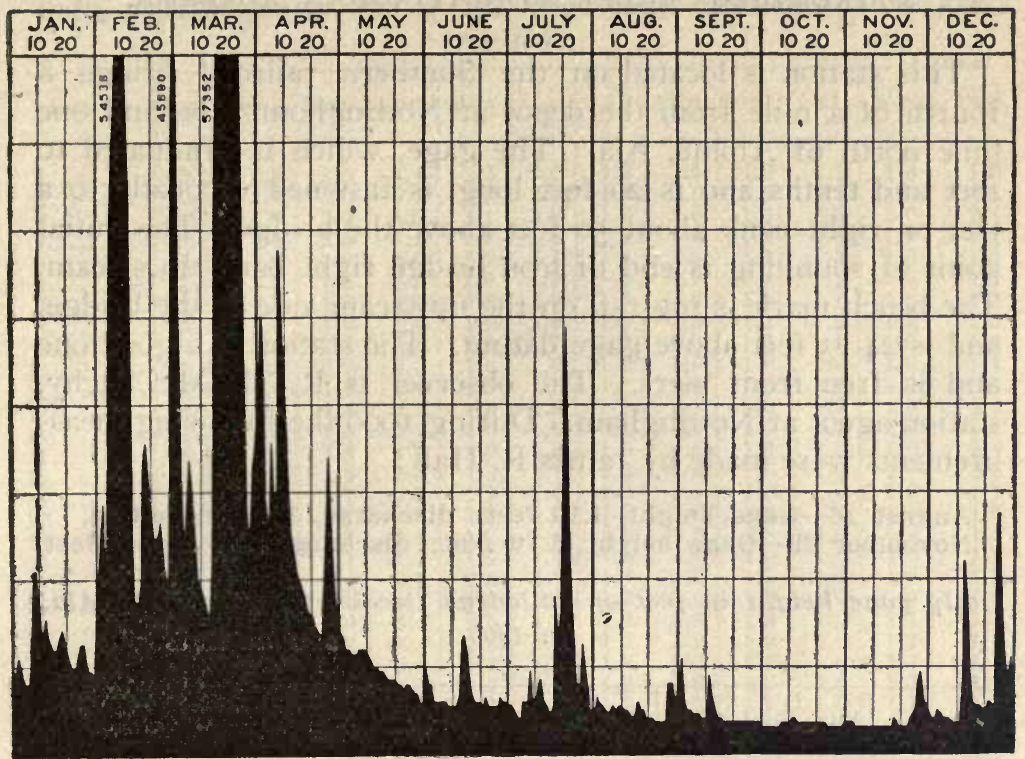

Fig. 13-Discharge of Coosa River at Rome, Ga., 1899.

Minimum monthly discharge of Coosa River at Rome, Ga., with corresponding net horse-power per foot of fall on a water utieel realizing 80 per cent. of the theoretical power.

\begin{tabular}{|c|c|c|c|c|c|c|c|c|c|}
\hline & \multicolumn{3}{|c|}{1899} & \multicolumn{3}{|c|}{1900} & \multicolumn{3}{|c|}{1901} \\
\hline & 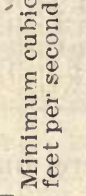 & 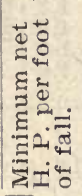 & 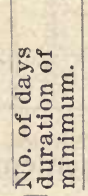 & 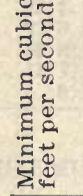 & 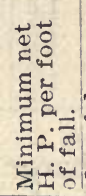 & & 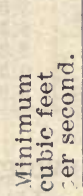 & 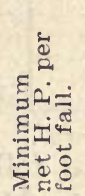 & 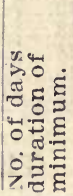 \\
\hline January & 4,060 & 369 & 2 & 2,725 & 248 & 8 & 4,600 & 418 & 1 \\
\hline February. & 8,710 & 792 & 2 & 2,850 & 259 & 1 & 4,900 & 445 & 1 \\
\hline March .... & 7.705 & 700 & 1 & 6,300 & 573 & 1 & 4,600 & 418 & 1 \\
\hline April ..... & 6,700 & 609 & 1 & 5,755 & 523 & 1 & 6,901 & 627 & 1 \\
\hline May ....... & 3,100 & 282 & 2 & 3,850 & 350 & 1 & 4,150 & 377 & 2 \\
\hline June ..... & 2,740 & 249 & 4 & 4,000 & 364 & 1 & 4,450 & 405 & 2 \\
\hline July ...... & 1,950 & 177 & 1 & 4,000 & 364 & 3 & 2,850 & 259 & 1 \\
\hline August $\ldots$ & 1,790 & 163 & 3 & 2,725 & 248 & 3 & 2,850 & 259 & 2 \\
\hline September & 1,550 & 141 & 1 & 1,930 & 175 & 5 & 3,120 & 284 & 1 \\
\hline October ... & 1,470 & 134 & 4 & 2,010 & 183 & 3 & 2,350 & 214 & 1 \\
\hline November & 1,470 & 134 & 7 & 2,600 & 236 & 2 & 2,110 & 192 & 10 \\
\hline December . & 1,870 & 170 & 4 & 3,400 & 309 & 2 & 2,475 & 225 & 3 \\
\hline
\end{tabular}

Note. - To find the minimum net horse power available at a shoal on this stream, near this station, for any month, multiply the total fall of the shoal by the "Net H. P. per foot of fall" in this table for that month. 


\section{TALLADEGA CREEK AT NOTTINGHAM, ALABAMA.}

This station is located on the Southern railroad bridge a fourth of a mile from the depot at Nottinghom, Ala, and one mile north of Alpine, Ala. The gage, which is graduated to feet and tenths and is 20 feet long, is fastened vertically to a tree on right bank about 50 feet above the bridge. The initial point of sounding is end of iron bridge right bank up stream. The bench mark is top rail on the upstream side of the bridge, and is 24.13 feet above gage datum. The station is a good one and is free from piers. The observer is R. M. McClatchy, station agent at Nottingham. During 1900 the following measurements were made by James R. Hall:

August 16-Gage height, 1.10 feet; distharge, 102 second-feet.

November 29-Gage height, 1.70 feet; discharge, 240 second-feet.

Daily gage height in feet of Talladega Creek at Nottingham, Ala., for 1900.

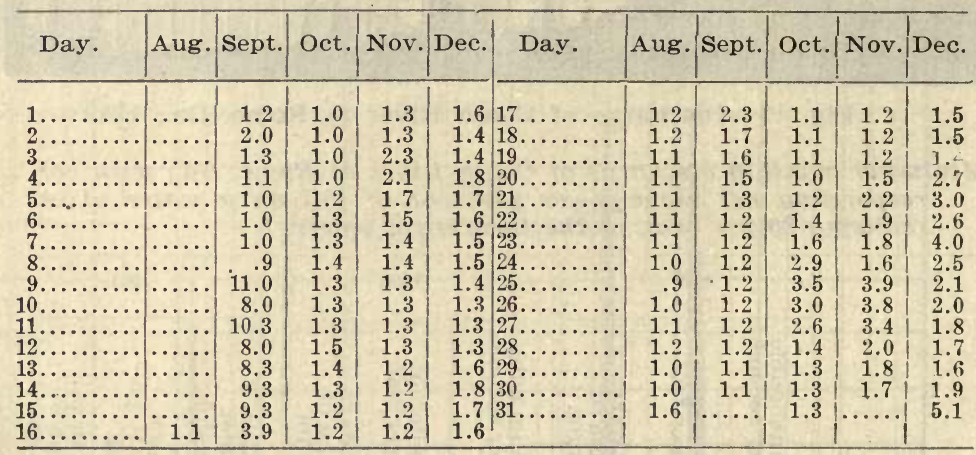

The following discharge measurements were made during I90 I by Max Hall and others:

April 5-Gage height, 3.0 feet; discharge, 526 second-feet. October 22-Gage height, 1.0 feet; discharge, 90 second-feet. 
Ecily gage height in feet of Talladega Cre€k at Nottingham, Ala., for 1901.

\begin{tabular}{|c|c|c|c|c|c|c|c|c|c|c|c|c|}
\hline Day & Jan. & Feb. & Mar. & April & May & Jun. & July & Aug. & Sept. & Oct. & Nov. & Dec \\
\hline & 3.2 & 2.3 & 2.0 & 3.4 & 2.2 & 2.0 & 1.5 & 1.1 & 1.2 & 1.1 & 1.0 & 1.0 \\
\hline & 2.8 & 2.1 & 2.0 & 5.9 & 2.2 & 2.0 & 1.4 & 1.0 & 1.2 & 2.8 & 1.0 & 1.4 \\
\hline 3... & 3.2 & 3.8 & 2.0 & 4.5 & 2.1 & 2.0 & 1.3 & 1.0 & 1.2 & 2.5 & 1.0 & 1.3 \\
\hline & 2.7 & 8.0 & 2.0 & 3.4 & 2.0 & 1.8 & 1.3 & 1.0 & 1.1 & 1.5 & 1.0 & 1.2 \\
\hline $5 \ldots$ & 2.4 & 3.9 & 1. & 3. & 2.0 & 1.7 & 1.3 & 1.0 & 1.1 & 1.3 & 1.0 & 1.2 \\
\hline & 2.2 & 3.2 & 1.8 & 2.9 & 1.9 & 1.7 & 1.4 & 1.0 & 1.1 & 1.2 & 1.0 & 1.3 \\
\hline & 2.1 & 2.8 & 1. & 2. & 1.9 & 3.0 & 1.1 & 1.0 & 1.1 & 1.1 & 1.0 & 1.1 \\
\hline & 2.1 & 2.8 & 1.8 & 2.5 & $1 . y$ & 1.9 & 1.3 & 1.0 & 1.1 & 1.1 & 1.0 & 1.0 \\
\hline $9 \ldots$ & 2.0 & 3.3 & 1. & 2.3 & 1.9 & 1.7 & 1.3 & 1.0 & 1.0 & 1.1 & 1.0 & 1.0 \\
\hline $10 .$. & 1.9 & 2.8 & 2.0 & 2.2 & 1.9 & 1.6 & 1.2 & 1.0 & 1.0 & 1.0 & 1.0 & 1. \\
\hline $11 .$. & 5.8 & 2. & 1.9 & 2.1 & 1.9 & 1.5 & 1.2 & 1.0 & 1. & 1. & 1.0 & 1. \\
\hline $12 .$. & 8.8 & 2.7 & 1.8 & 2.1 & 1.9 & 1.5 & 1.2 & 1.1 & 1.0 & 1.0 & 1.0 & 1. \\
\hline $13 \ldots$ & 4.7 & 2.5 & 1.8 & 2.7 & 2.0 & 1.6 & 1.2 & 1.0 & 1. & 1.1 & 1.0 & 1. \\
\hline 14. & 3.4 & 2.3 & 1.8 & 3.7 & 1.9 & 1.8 & 1.2 & 1.0 & 1.0 & 1.0 & 1. & 2 . \\
\hline 15. & 2.9 & 2.2 & $1.8^{\circ}$ & 2.7 & 19 & 1.7 & 1.2 & 1.0 & 1. & 1.0 & 1.0 & 3. \\
\hline 16. & 2.6 & 2.2 & 1.7 & 2.5 & 1.9 & 1.6 & 1.2 & 2.3 & 1.0 & 1.0 & 1.0 & 1.7 \\
\hline 17. & 2.7 & 2.2 & 1.7 & 2.3 & 1.8 & 1.6 & 1.2 & 2.2 & 3. & 1. & 1.0 & 1. \\
\hline 18 & 2.4 & 2.1 & 1.7 & 2.4 & 1.7 & & 2.0 & 1.9 & 2. & 1.0 & 1.0 & 1. \\
\hline 19. & 2. & 2.1 & 1.7 & 11.2 & 1.9 & 1. & 1.5 & 1.7 & 1. & 1. & 1.0 & 1. \\
\hline 20. & 2.2 & 2.0 & 1.8 & 6.3 & 2.5 & 1. & 1. & 1.5 & 1.5 & 1.0 & 1.0 & 1. \\
\hline & 2.1 & 2.0 & 2. & 4.1 & 3. & 1. & 1. & 1.4 & 1.4 & & & 1. \\
\hline & 2.2 & 2.0 & 2.0 & 3.4 & 2.5 & 1. & 1.2 & 1.3 & 1. & 1.0 & 1.1 & 1. \\
\hline 23 . & 2.2 & 2.0 & 1. & 3.1 & 2. & 1. & 1.2 & 1.2 & 1. & & 1.2 & 1. \\
\hline & 2.2 & 2.1 & 2.6 & 2.8 & 1.8 & 1. & 1.2 & 1.2 & 1.2 & 1.0 & 1.3 & 1. \\
\hline & 2.2 & 2.1 & 2. & 2.7 & 1. & 1. & 1.2 & 1.5 & 1. & & 1.2 & 1. \\
\hline & 2.3 & 2.1 & 8.9 & 2.6 & 1.7 & 1. & 1.2 & 1.3 & 1. & 1.0 & 1.1 & 1.2 \\
\hline & 2.2 & 2.0 & 4. & 2.5 & 1.7 & 1. & 1.2 & 1.4 & 1. & 1.0 & 1.1 & 1. \\
\hline & 2.2 & .20 & 3. & 2.4 & 1. & 1.3 & 1.2 & 1.5 & 1.1 & 1.0 & 1.0 & 1.2 \\
\hline & 2.2 & & 2.8 & 2.3 & 1. & 1.3 & 1. & 1.4 & 1.1 & 1.0 & 1.0 & \\
\hline & 2.6 & & 2.9 & 2.2 & 1.7 & 1.4 & 1.2 & 1.3 & 1.1 & 1.0 & 1.0 & 7. \\
\hline $31 \ldots \ldots \ldots$ & 2.5 & $1 \cdots \cdots \cdots$ & 5.5 & $|\cdots \cdots \cdot|$ & 2.2 & ... & 1.2 & 1.2 & $1 \cdots \cdots \cdots$ & 1.0 & & \\
\hline
\end{tabular}

Rating table for Talladega Creek at Nottingham, Ala., for

\begin{tabular}{|c|c|c|c|c|c|c|c|}
\hline 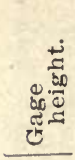 & 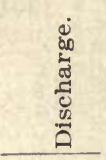 & 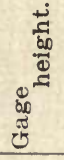 & 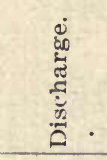 & 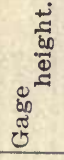 & 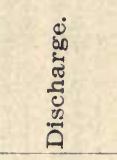 & 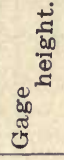 & 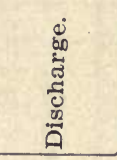 \\
\hline $\mathrm{Fee}^{\imath}$. & Second $f t$. & Feet. & Second $f \prime$. & Feet. & Second ft. & Feet. & Second ft. \\
\hline 1.0 & 90 & 2.1 & 328 & 3.2 & 570 & 4.3 & 812 \\
\hline 1.1 & 109 & 2.2 & 350 & 3.3 & 592 & 4.4 & 834 \\
\hline 1.2 & 130 & 2.3 & 372 & 3.4 & 614 & 4.5 & 856 \\
\hline 1.3 & 152 & 2.4 & 394 & 3.5 & 636 . & 4.6 & 878 \\
\hline 1.4 & 174 & 2.5 & 416 & 3.6 & 658 & 4.7 & 900 \\
\hline 1.5 & 196 & 2.6 & 438 & 3.7 & 680 & 4.8 & 922 \\
\hline 1.6 & 218 & 2.7 & 460 & 3.8 & 702 & 4.9 & 944 \\
\hline 1.7 & 240 & 2.8 & 482 & 3.9 & 724 & 5.0 & 966 \\
\hline 1.8 & 262 & 2.9 & 504 & 4.0 & 746 & & \\
\hline 1.9 & 284 & 3,0 & 526 & 4.1 & 768 & & \\
\hline 2.0 & 306 & 3.1 & 548 & 4.2 & 790 & & \\
\hline
\end{tabular}

NotE.-This table applied to the foregoing "Daily gage heights" gives the cubic feet per second flowing in the river on each date for which the gage height is given. 
Estimated monthly discharge of Talladega Creek at Nottingham, Ala.

\begin{tabular}{|c|c|c|c|c|c|}
\hline \multirow[b]{2}{*}{ Month. } & \multicolumn{3}{|c|}{$\begin{array}{l}\text { Discharge in } \\
\text { second-feet. }\end{array}$} & \multicolumn{2}{|c|}{ Run-off. } \\
\hline & $\begin{array}{l}\text { Maxi- } \\
\text { mum. }\end{array}$ & $\begin{array}{l}\text { Mini- } \\
\text { mum. }\end{array}$ & Mean. & $\begin{array}{l}\text { Second- } \\
\text { feet per } \\
\text { square } \\
\text { mile. }\end{array}$ & $\begin{array}{l}\text { Depth } \\
\text { in } \\
\text { inches. }\end{array}$ \\
\hline 1900. & & & & & \\
\hline August $16-31$ & & & 113 & 0.72 & 0.43 \\
\hline September ...... & 2,286 & 74 & 575 & 3.69 & 4.12 \\
\hline October $\ldots \ldots \ldots \ldots \ldots \ldots$ & 636 & 90 & 190 & 1.22 & 1.41 \\
\hline November $\ldots \ldots \ldots \ldots \ldots \ldots$ & 724 & 130 & 249 & 1.60 & 1.79 \\
\hline December & 746 & 152 & 291 & 1.87 & 2.16 \\
\hline 1901. & & & & & \\
\hline January . & 1,802 & 284 & 485 & 3.11 & 3.59 \\
\hline February $\ldots \ldots \ldots \ldots \ldots \ldots$ & 1,626 & 306 & 449 & 2.88 & 3.00 \\
\hline March $\ldots \ldots \ldots \ldots \ldots \ldots \ldots$ & 1,824 & 240 & 405 & 2.60 & 3.00 \\
\hline April $\ldots \ldots \ldots \ldots \ldots \ldots \ldots$ & 2,330 & 328 & 591 & 3.79 & 4.23 \\
\hline May ........ & 724 & 240 & 306 & 1.96 & 2.26 \\
\hline June $\ldots \ldots \ldots \ldots \ldots \ldots \ldots$ & 526 & 152 & 218 & 1.40 & 1.56 \\
\hline July $\ldots \ldots \ldots \ldots \ldots \ldots \ldots$ & 196 & 130 & 149 & .96 & 1.11 \\
\hline August $\ldots \ldots \ldots \ldots \ldots \ldots \ldots$ & 372 & 90 & 148 & .95 & 1.10 \\
\hline September $\ldots \ldots \ldots \ldots \ldots \ldots$ & 614 & 90 & 148 & .95 & 1.06 \\
\hline October $\ldots \ldots \ldots \ldots \ldots \ldots$ & 482 & 90 & 123 & .79 & .91 \\
\hline November $\ldots \ldots \ldots \ldots \ldots \ldots$ & 152 & 90 & 97 & .62 & .69 \\
\hline December ........... & 1,714 & 90 & 264 & 1.69 & 1.95 \\
\hline The year $\ldots \ldots \ldots \ldots \ldots \ldots$ & 2,330 & 90 & 282 & 1.81 & 24.46 \\
\hline
\end{tabular}

Minimum monthly discharge of Talladega Creek at Nottingham, Ala., with corresponding net horse-power per foot of fall on a waterwheel realizing 80 per cent. of the theoretical power.

\begin{tabular}{|c|c|c|c|c|c|c|}
\hline & \multicolumn{3}{|c|}{1900} & \multicolumn{3}{|c|}{1901} \\
\hline & 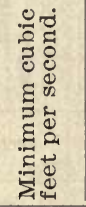 & 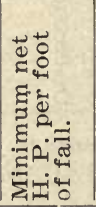 & 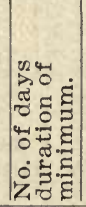 & 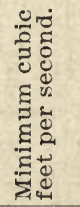 & 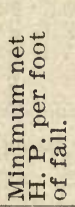 & 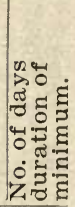 \\
\hline$\overline{\text { January } \ldots \ldots \ldots \ldots \ldots \ldots \ldots}$ & $\ldots \ldots$ & $\ldots \ldots$ & $.1 \ldots$. & 284 & 26 & 1 \\
\hline February $\ldots \ldots \ldots \ldots \ldots \ldots$ & $\ldots .$. & $\ldots \ldots$ &.$\ldots$ & 306 & 28 & 6 \\
\hline March $\ldots \ldots \ldots \ldots \ldots \ldots \ldots$ & .... & $\ldots \ldots$ & $\ldots$ & 240 & 22 & 4 \\
\hline April $\ldots \ldots \ldots \ldots \ldots \ldots \ldots$ & ... & $\therefore . .$. & $\ldots$ & 328 & 30 & 2 \\
\hline May $\ldots \ldots \ldots \ldots \ldots \ldots \ldots \ldots$ & & $\ldots$. & $\ldots$ & 240 & 22 & 7 \\
\hline June $\ldots \ldots \ldots \ldots \ldots \ldots \ldots \ldots$ & & $\ldots \ldots$ & $\ldots$ & 152 & 14 & 8 \\
\hline July $\ldots \ldots \ldots \ldots \ldots \ldots \ldots$ & & $\ldots \ldots$ & & 130 & 12 & 18 \\
\hline August $\ldots \ldots \ldots \ldots \ldots \ldots \ldots$ & 66 & 6 & 1 & 90 & 8 & 13 \\
\hline September $\ldots \ldots \ldots \ldots \ldots \ldots$ & 74 & 6.7 & 1 & 90 & 8 & 8 \\
\hline October $\ldots \ldots \ldots \ldots \ldots \ldots \ldots$ & 90 & 8 & 5 & 90 & 8 & 21 \\
\hline November ............ & 130 & 12 & 7 & 90 & 8 & 24 \\
\hline December $\ldots \ldots \ldots \ldots \ldots \ldots \ldots$ & 152 & 14 & 3 & 90 & 8 & $\mathbf{5}$ \\
\hline
\end{tabular}

NoTE.-To find the minimum net horse power available at a shoal on this stream, near this station, for any month, multiply the total fall of the shoal by the "Net H. P. per foot of fall" in this table for that month. 


\section{ALABAMA RIVER AT SELMA, ALABAMA.}

This station was originally established by the United States Engineer Corps; readings are now taken by the United States Weather Bureau. The gage, which is attached to the iron highway bridge, the floor of which is about 60 feet above low water, is in two sections. The lower section, which reads from -0.3 feet to +2.30 feet, is secured to the pile on the lower side of the cofferdam on the draw pier; the upper section, which reads from 2.30 feet to 48 feet, is spiked to the highway bridge. The bench mark, which is an iron boit driven into the face of a rock bluff 182.3 feet from the first bridge pier, on the road ascending to the city, is 26 feet above the zero of the gage and 87.30 feet above mean sea level. The top of the coping stone of the pivot pier at the highway bridge to which gage is attached is 56 feet above the zero of the gage, and II 7.30 feet above mean sea level. Graduations extend from -3.0 feet to +48 feet. No measurements of discharge were made here during 1899 .

Daily gage height, in feet, of Alabama River at Selma, Ala., for 1899.

\begin{tabular}{r|r|r|r|r|r|r|r|r|r|r|r}
\hline Day & Jan. & Feb. & Mar. & April & May & Jun. & July & Aug. & Sept. & Oct. & Nov.| \\
\hline
\end{tabular}


During I900 the following measurements were made:

April 14-Gage height, 23.60 feet; discharge 66,607 second-ieet. May 26-Gage height, 6.10 feet; discharge, 17,049 second-teet.

August 24-Gage height, 3.10 feet; discharge, 9,879 second-feet.

Daily gage height, in feet, of Alabama River at Selma, Ala., for 1900.

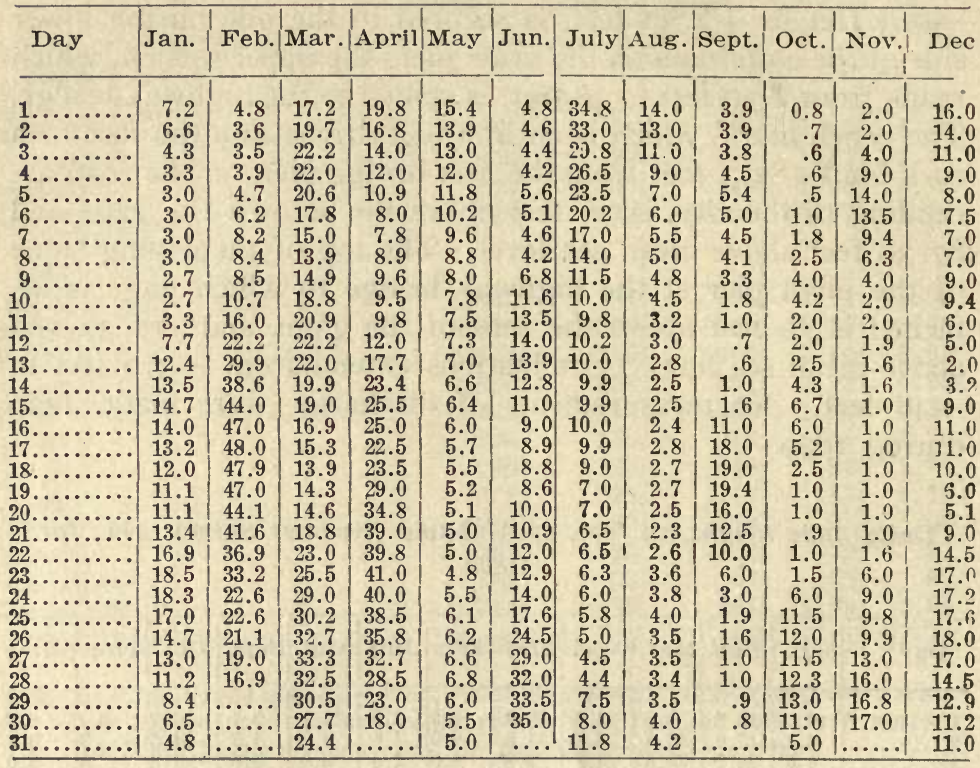


List of Discharge Measurements made on Alabama River at Selma, Alabama, in 1901.

\begin{tabular}{|c|c|c|c|}
\hline Date, 1901. & Hydrographer. & $\begin{array}{c}\text { Gage } \\
\text { height, ft }\end{array}$ & $\begin{array}{l}\text { Dischg. } \\
\text { sec.-ft }\end{array}$ \\
\hline March 14. & Max Hall & 14.20 & 35,518 \\
\hline April 25. . & J. R. Hall . & 34.00 & 90,332 \\
\hline August $9 .$. & K. T. Thomas ............... & 4.35 & 12,519 \\
\hline October 30 & Max Hall $\ldots \ldots \ldots \ldots \ldots \ldots \ldots \ldots$ & 1.10 & 7,710 \\
\hline
\end{tabular}

Daily gage height, in feet, of Alabama River at Selma, Ala., for 1901.

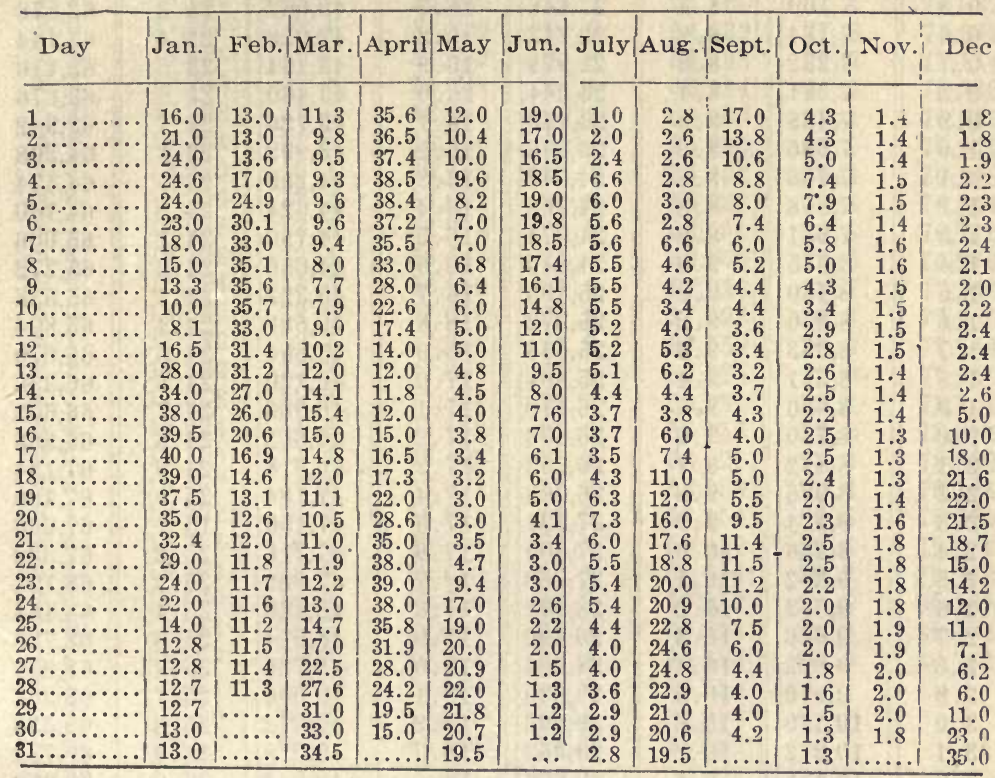


Rating table for Alabama River at Selma, Ala., for $190 \dot{0}$ and 1901.

\begin{tabular}{|c|c|c|c|c|c|c|c|}
\hline 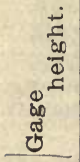 & 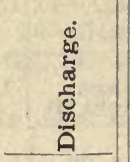 & 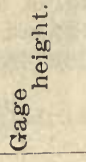 & 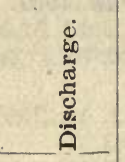 & 赵 & 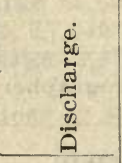 & 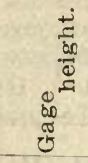 & 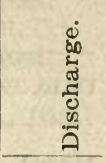 \\
\hline Feet, & Second ft. & Feet. & Second ft. & Feet. & Second $f t$. & Feet. & Second $f t$ \\
\hline 0.0 & 6,700 & 7.6 & 21,116 & 15.2 & 41,332 & 22.8 & 61,548 \\
\hline 0.1 & 6,770 & 7.7 & 21,382 & 15.3 & 41,598 & 22.9 & 61,814 \\
\hline 0.2 & 6,845 & 7.8 & 21,648 & 15.4 & 41,864 & 23.0 & 62,080 \\
\hline 0.3 & 6,925 & 7.9 & 21,914 & 15.5 & 42,130 & 23.1 & 62,346 \\
\hline 0.4 & 7,010 & 8.0 & 22,180 & 15.6 & 42,396 & 23.2 & 62,612 \\
\hline $0 . \overline{5}$ & 7,100 & 8.1 & 22,446 & 15.7 & 42,662 & 23.3 & 62,878 \\
\hline 0.6 & 7,184 & 8.2 & 22,712 & 15.8 & 42,928 & 23.4 & 63,144 \\
\hline 0.7 & 7,282 & 8.3 & 22,978 & 15.9 & 43,194 & 23.5 & 63,410 \\
\hline 0.8 & 7,384 & 8.4 & 23,244 & 16.0 & 43,460 & 23.6 & 63,676 \\
\hline 0.9 & 7,488 & 8.5 & 23,510 & 16.1 & 43,726 & 23.7 & 63,942 \\
\hline 1.0 & 7,596 & 8.6 & 23,776 & 16.2 & 43,992 & 23.8 & 64,208 \\
\hline 1.1 & 7,706 & 8.7 & 24,042 & 16.3 & 44,258 & 23.9 & 64,474 \\
\hline 1.2 & 7,818 & 8.8 & 24,308 & 16.4 & 44,524 & 24.0 & 64,740 \\
\hline 1.3 & 7,931 & 8.9 & 24,574 & 16.5 & 44,790 & 24.1 & 65,006 \\
\hline 1.4 & 8,045 & 9.0 & 24,840 & 16.6 & 44,056 & 24.2 & 65,272 \\
\hline 1.5 & 8,160 & 9.0 & 25,106 & 16.7 & 45,322 & 24.3 & 65,538 \\
\hline 1.6 & 8,270 & 9.2 & 25,372 & 16.8 & 45,588 & 24.4 & 65,804 \\
\hline 1.7 & 8,393 & 9.3 & 25,638 & 16.9 & 45,854 & 24.5 & 66,070 \\
\hline 1.8 & 8,511 & 9.4 & 25,904 & 17.0 & 46,120 & 24.6 & 66,336 \\
\hline 1.9 & 8,630 & 9.5 & 26,170 & 17.1 & 46,386 & 24.7 & 66,602 \\
\hline 2.0 & 8,750 & 9.6 & 26,436 & 17.2 & 46,652 & 24.8 & 66,868 \\
\hline 2.1 & 8,872 & 9.7 & 26,702 & 17.3 & 46,918 & 24.9 & 67,134 \\
\hline 2.2 & 8,996 & 9.8 & 26,968 & 17.4 & 47,184 & 25.0 & 67,400 \\
\hline 2.3 & 9,124 & 9.9 & 27,234 & 17.5 & 47,450 & 15.1 & 67,666 \\
\hline $2.4^{\circ}$ & 9,256 & 10.0 & 27,500 & 17.6 & 47,716 & 25.2 & 67,932 \\
\hline 2.5 & 9,392 & 10.1 & 27,760 & 17.7 & 47,982 & 25.3 & 68,198 \\
\hline 2.6 & 9,532 & 10.2 & 28,032 & 17.8 & 48,248 & 25.4 & 68,464 \\
\hline 2.7 & 9,676 & 10.3 & 28,290 & 17.9 & 48,514 & 25.5 & 68,730 \\
\hline 2.8 & 9,822 & 10.4 & 28,564 & 18.0 & 48,780 & 25.6 & 68,996 \\
\hline 2.9 & 9,970 & 10.5 & 28,830 & 18.1 & 49,046 & 25.7 & 69,262 \\
\hline 3.0 & 10,120 & 10.6 & 29,096 & 18.2 & 49,312 & 25.8 & 69,528 \\
\hline 3.1 & 10,272 & 10.7 & 29,362 & 18.3 & 49,578 & 25.9 & 69,794 \\
\hline 3.2 & 10,428 & 10.8 & 29,628 & 18.4 & 49,844 & 26.0 & 70,060 \\
\hline 3.3 & 10,588 & 10.9 & 29,894 & 18.5 & 50,110 & 26.1 & 70,326 \\
\hline 3.4 & 10,752 & 11.0 & 30,160 & 18.6 & 50,376 & 26.2 & 70,592 \\
\hline 3.5 & 10,920 & 11.1 & 30,426 & 18.7 & 50,642 & 26.3 & 70,858 \\
\hline 3.6 & 11,092 & 11.2 & 30,692 & 18.8 & 50,908 & 26.4 & 71,124 \\
\hline 3.7 & 11,268 & 11.3 & 30,958 & 18.9 & 51,174 & 26.5 & 71,390 \\
\hline 3.8 & 11,448 & 11.4 & 31,224 & 19.0 & 51,440 & 26.6 & 71,656 \\
\hline 3.9 & 11,632 & 11.5 & 31,490 & 19.1 & 51,706 & 26.7 & 71,922 \\
\hline 4.0 & 11,820 & 11.6 & 31,756 & 19.2 & 51,972 & 26.8 & 72,188 \\
\hline 4.1 & 12,015 & 11.7 & 32,022 & 19.3 & 52,238 & 26.9 & 72,454 \\
\hline 4.2 & 12,220 & 11.8 & 32,228 & 19.4 & 52,504 & 27.0 & 72,720 \\
\hline 4.3 & 12,435 & 11.9 & 32,556 & 19.5 & 52,770 & 27.1 & 72,986 \\
\hline 4.4 & 12,660 & 12.0 & 32,820 & 19.6 & 53,036 & 27.2 & 73,252 \\
\hline 4.5 & 12,900 & 12.1 & 33,086 & 19.7 & 53,302 & 27.3 & 73,518 \\
\hline
\end{tabular}


Rating table for Alabama River at Selma, Ala., for 1900 and 1901. Continued.

\begin{tabular}{|c|c|c|c|c|c|c|c|}
\hline 点 & 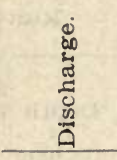 & 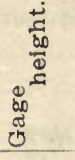 & 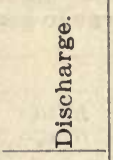 & 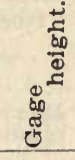 & 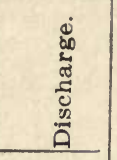 & 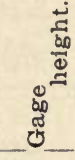 & 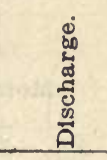 \\
\hline Feet. & Seeond $f t$. & Feet. & Second $f t$ & Feet. & Second $f t$. & Feet & Second $f t$. \\
\hline 4.6 & 13,150 & 12.2 & 33,352 & 19.8 & 53,568 & 27.4 & 73,784 \\
\hline 4.7 & 13,405 & 12.3 & 33,618 & 19.9 & 53,884 & 27.5 & 74,050 \\
\hline 4.8 & 13,668 & 12.4 & 33,884 & 20.0 & 54,100 & 27.6 . & 74,316 \\
\hline 4.9 & 13,934 & 12.5 & 34,150 & 20.1 & 54,366 & 27.7 & 74,582 \\
\hline 5.0 & 14,200 & 12.6 & 34,416 & 20.2 & 54,632 & 27.8 & 74,848 \\
\hline 5.1 & 14,466 & 127. & 34,682 & 20.3 & 54,898 & 27.9 & 75,114 \\
\hline 5.2 & 14,732 & 12.8 & 34,948 & 20.4 & 55,164 & 28.0 & 75,380 \\
\hline 5.3 & 14,998 & 12.9 & 35,214 & 20.5 & 55,430 & 28.1 & 75,646 \\
\hline 5.4 & 15,264 & 13.0 & 35,480 & 20.6 & 55,696 & 28.2 & 75,912 \\
\hline 5.5 & 15,530 & 13.1 & 35,746 & 20.7 & 55,962 & 28.3 & 76,178 \\
\hline 5.6 & 15,796 & 13.2 & 36,012 & 20.8 & 56,228 & 28.4 & 76,444 \\
\hline 5.7 & 16,062 & 13.3 & 36,278 & 20.9 & 56,494 & 28.5 & 76,710 \\
\hline 5.8 & 16,328 & 13.4 & 36,544 & 21.0 & 56,760 & 28.6 & 76,976 \\
\hline 5.9 & 16,594 & 13.5 & 36,810 & 21.1 & 57,026 & 28.7 & 77,242 \\
\hline 6.0 & 16,860 & 13.6 & 37,076 & 21.2 & 57,292 & 28.8 & 77,508 \\
\hline 6.1 & 17,126 & 13.7 & 37,342 & 21.3 & 57,558 & 28.9 & 77,744 \\
\hline 6.2 & 17,392 & 13.8 & 37,608 & 21.4 & 57,824 & 29.0 & 78,040 \\
\hline 6.3 & 17,658 & 13.9 & 37,874 & 21.5 & 58,090 & 29.1 & 78,306 \\
\hline 6.4 & 17,924 & 14.0 & 38,140 & 21.6 & 58,356 & 29.2 & 78,572 \\
\hline 6.5 & 18,190 & 14.1 & 38,406 & 21.7 & 58,622 & 29.3 & 78,838 \\
\hline 6.6 & 18,456 & 14.2 & 38,672 & 21.8 & 58,888 & 29.4 & 79,104 \\
\hline 6.7 & 18,722 & 14.3 & 38,838 & 21.9 & 59,154 & 29.5 & 79,370 \\
\hline 6.8 & 18,988 & 14.4 & 39,104 & 22.0 & 59,420 & 29.6 & 79,636 \\
\hline 6.9 & 19,254 & 14.5 & 39,370 & 22.1 & 59,686 & 29.7 & 79,902 \\
\hline 7.0 & 19,520 & 14.6 & 39,676 & 22.2 & 59,952 & 29.8 & 80,168 \\
\hline 7.1 & 19,786 & 14.7 & 40,002 & 22.3 & 60,218 & 29.9 & 80,434 \\
\hline 7.2 & 20,052 & 14.8 & 40,268 & 22.4 & 60,484 & 30.0 & 80,700 \\
\hline 7.3 & 20,318 & 14.9 & 40,534 & 22.5 & 60,750 & & \\
\hline 7.4 & 20,584 & 15.0 & 40,800 & 22.6 & 61,016 & & \\
\hline 7.5 & 20,850 & 15.1 & 41,066 & 22.7 & 61,282 & & \\
\hline
\end{tabular}

Note-This table applied to the foregoing "daily gage heights" gives cubic feet per second flowing in the river on each date for which the gage height is given. 
Estimated monthly discinarge of Alabama River at Selma. Ala. [Drainage area, 13,500 square miles.]

\begin{tabular}{|c|c|c|c|c|c|}
\hline \multirow[b]{2}{*}{ Month. } & \multicolumn{3}{|c|}{ Discharge in second-feet } & \multicolumn{2}{|c|}{ Run-off. } \\
\hline & $\begin{array}{l}\text { Maxi- } \\
\text { mum. }\end{array}$ & $\begin{array}{l}\text { Mini- } \\
\text { mutn. }\end{array}$ & Mean. & $\begin{array}{l}\text { Depth } \\
\text { in } \\
\text { inches. }\end{array}$ & $\begin{array}{l}\text { Second. } \\
\text { feet per } \\
\text { square } \\
\text { mile. }\end{array}$ \\
\hline 1900. & & & & & \\
\hline January & 50,110 & 9,676 & 26,495 & 1.96 & 2.2 \\
\hline ebruary & 128,540 & 10,920 & & 4.72 & 4.91 \\
\hline March .. & 89,478 & 37 , & 72 & 4.32 & 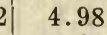 \\
\hline$\ldots \ldots \ldots \ldots$ & 109,96 & & 09 & 4.51 & 5.0 \\
\hline May $\ldots$. & 41,8 & & & 1.56 & 1.80 \\
\hline June & 94,000 & 12,2 & 35,288 & 2.61 & 2.91 \\
\hline$\ldots \ldots \ldots \ldots$ & 93,4 & 12,660 & 33,964 & 2.52 & 2.90 \\
\hline August ... & 38 , & & & 1.05 & 1.21 \\
\hline September & 52,504 & 7,1 & 17,366 & 1.29 & 1.44 \\
\hline ctober $\quad . . . . . . . .$. & & & & 1. & 1.23 \\
\hline November & 46 , & & & 1. & 1.5 \\
\hline December . & 48,780 & 8,750 & 28,989 & 2.15 & 2.4 \\
\hline The year $\ldots \ldots \ldots \ldots$ & 128,540 & 7,097 & 33,772 & 2.34 & 32.68 \\
\hline & & & & & \\
\hline ry . & 107,300 & 22,446 & 61,213 & 4.53 & 5.2 \\
\hline February & 95,8 & & 37 & 4. & 4.2 \\
\hline March ... & & & & & 3.3 \\
\hline pril . & 104,64 & & 73,048 & 5.41 & 6.0 \\
\hline May & 59,4 & 10,120 & 26,966 & 2.00 & 2.31 \\
\hline June .. & 53,568 & 7,818 & 26,030 & 1.93 & 2.1 \\
\hline & & 7,5 & 13,536 & 1.00 & 1.15 \\
\hline ust $\ldots$.. & 66,868 & 9,532 & 30,853 & 2.29 & 2.6 \\
\hline September & 46,120 & 10,428 & 19,394 & 1.44 & 1.6 \\
\hline & & & & .82 & .9 \\
\hline November & 8,7 & 7,931 & 8,266 & .61 & .68 \\
\hline December $\ldots \ldots \ldots \ldots \ldots$ & 94,000 & 8,511 & 26,638 & 1.97 & 2.27 \\
\hline The year $\ldots \ldots \ldots \ldots$ & 107,300 & 7,596 & 32,585 & 2.47 & 32.60 \\
\hline
\end{tabular}


Minimum monthly discharge of the Alabama River at Selma, Ala., with corresponding net horsepower per foot of fall on a water wheel realizing 80 per cent. of the theoretical power.

[Drainage area, 15,400 square miles.]

\begin{tabular}{|c|c|c|c|c|c|c|c|c|c|}
\hline & & 1899. & & & 1300. & & & 1901 & \\
\hline & 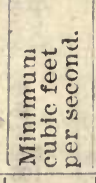 & 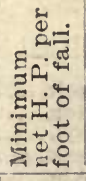 & & 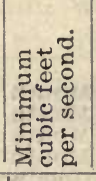 & 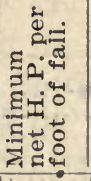 & 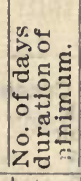 & 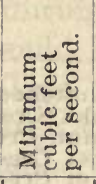 & 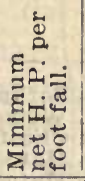 & 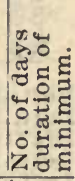 \\
\hline January & 16,328 & 1,484 & 1 & 9,676 & 880 & 2 & 22,446 & 2,041 & \\
\hline February & 29,628 & 2,693 & 1 & 10,920 & 993 & 1 & 30,692 & 2,790 & 5 \\
\hline March ... & $37,874 \mid$ & 3,443 & 1 & 37,874 & 3,443 & 2 & 21,382 & 1,944 & $t$ \\
\hline April .. & 27,760 & 2,524 & 1 & 21,648 & 1,968 & 1 & 32,288 & 2,935 & 1 \\
\hline May . & 12,220 & 1,111 & 1 & 13,668 & 1,243 & 1 & 10,120 & 920 & 2 \\
\hline June .... & 8,045 & 731 & 2 & 12,220 & 1,111 & 2 & 7,818 & 711 & 2 \\
\hline July $\ldots \ldots \ldots$ & 7,184 & 653 & 2 & 12,660 & 1,151 & 1 & 7,596 & 691 & 1 \\
\hline August ... & 9,532 & 867 & 3 & 9,124 & 829 & 1 & 9,532 & 867 & 2 \\
\hline September & 5,800 & 527 & 2 & 7,189 & 653 & 1 & 10,428 & 948 & 1 \\
\hline October .... & & 491 & 1 & 7,100 & 645 & 1 & 7,931 & 721 & 2 \\
\hline November .. & 5,700 & 518 & 3 & 7,596 & 691 & 7 & 7,931 & 721 & 3 \\
\hline December .. & 7,931 & 721 & 2 & 8,750 & 795 & 1 & 8,511 & 774 & 1 \\
\hline
\end{tabular}

Note. - To find the minimum net horse power available at a shoal on this stream, near this station, for any month, multiply the total fall of the shoal by the "Net H. P. per foot of fall" in this table for that month.

\section{MISCELLANEOUS DISCHARGE MEASUREMENTS}

Made by B. M. Hall, and Assistants, on Tributaries of Coosa River.

1898.

May 26-Choccolocco Creek, Eureka; discharge, 171 second-feet; low water.

1900.

March 15-Talladega Creek, Kymulga postoffice; discharge, 107 second-feet; medium.

March 16-Tallassahatchee Creek, in Talladega county, Childersburg; discharge, 102 second-feet.

March 17-Hatchet Creek, Goodwater; discharge, 84 second-feet.

April 5-Choccolocco Creek, L. \& N. R. R. bridge, near Jenifer; discharge, 1,170 second-feet; high water.

Oot. 16-Big Wills Creek, Wesson's Mill, 2 miles north of Attalla; discharge, 107 second-feet; low water. 
6. TRIBUTARIES OF THE COOSA RIVER FROM WETUMPKA UP.

\begin{tabular}{|c|c|c|c|c|c|}
\hline 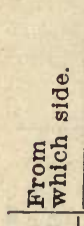 & Name of Stream. & Point on stream. & 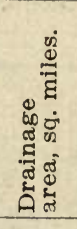 & 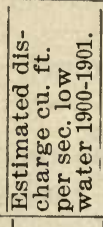 & 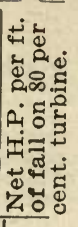 \\
\hline $\begin{array}{l}\text { Left.. } \\
\text { Left.. } \\
\text { Right } \\
\text { Left.. } \\
\text { Left.. } \\
\text { Left.. } \\
\text { Right } \\
\text { Right } \\
\text { Right } \\
\text { Left.. } \\
\text { Left.. } \\
\text { Left. } \\
\text { Right } \\
\text { Left.. } \\
\text { Left.. } \\
\text { Rigt. } \\
\text { Right } \\
\text { Left.. } \\
\text { Left.. } \\
\text { Left.. }\end{array}$ & 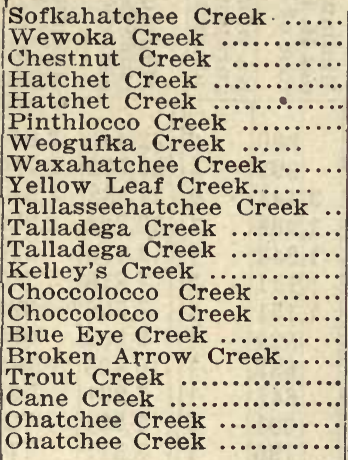 & $\begin{array}{l}\text { Mouth of Creek } \\
\text { Mouth of Creek } \\
\text { Mouth of Creek } \\
\text { Mouth of Creek } \\
\text { Goodwater, Ala. } \\
\text { Mouth of Creek. } \\
\text { Mouth of Creek } \\
\text { Mouth of Creek } \\
\text { Mouth of Creek } \\
\text { Mouth of Creek. } \\
\text { Mouth of Creek. } \\
\text { Nottingham. Ala } \\
\text { Mouth of Creek. } \\
\text { Mouth of Creek. } \\
\text { Jenifer, Ala.... } \\
\text { Mouth of Creek. } \\
\text { Mouth of Creek. } \\
\text { Mouth of Creek. } \\
\text { Mouth of Creek. } \\
\text { Mouth of Creek. } \\
\text { Above Tallassee }\end{array}$ & $\begin{array}{r}40 \\
85 \\
90 \\
500 \\
105 \\
60 \\
120 \\
196 \\
192 \\
172 \\
188 \\
156 \\
218 \\
510 \\
273 \\
26 \\
49 \\
23 \\
94 \\
217\end{array}$ & $\begin{array}{r}12 \\
28 \\
30 \\
165 \\
40 \\
24 \\
48 \\
75 \\
75 \\
70 \\
75 \\
66 \\
88 \\
153 \\
95 \\
7 \\
15 \\
10 \\
35 \\
85\end{array}$ & $\begin{array}{r}0.0 \\
6.8 \\
6.3 \\
6.8 \\
6.0 \\
8.0 \\
13.9 \\
8.6 \\
0.6 \\
1.6 \\
0.9 \\
3.2 \\
7.7\end{array}$ \\
\hline $\begin{array}{l}\text { Left. } \\
\text { Right } \\
\text { Right } \\
\text { Right } \\
\text { Right }\end{array}$ & $\begin{array}{l}\text { Tallasseehatchee Creek .. } \\
\text { Shoal Creek } \ldots \ldots \ldots \ldots \ldots \ldots \\
\text { Beaver Creek } \ldots \ldots \ldots \ldots \ldots \\
\text { Big Canoe Creek............. } \\
\text { Big Canoe Creek } \ldots \ldots \ldots \ldots\end{array}$ & $\begin{array}{l}\text { chee Crcek........... } \\
\text { Mouth of Creek...... } \\
\text { Mouth of Creek....... } \\
\text { Mouth of Creek...... } \\
\text { Mouth of Creek...... } \\
\text { Above Little Canoe }\end{array}$ & $\begin{array}{r}86 \\
125 \\
31 \\
33 \\
248\end{array}$ & $\begin{array}{l}35 \\
50 \\
12 \\
12 \\
90\end{array}$ & $\begin{array}{l}4 . \\
1 . \\
1 .\end{array}$ \\
\hline $\begin{array}{l}\text { Right } \\
\text { Right } \\
\text { Right }\end{array}$ & $\begin{array}{l}\text { Little Canoe Creek......... } \\
\text { Big Wills Creek } \ldots \ldots \ldots \ldots \\
\text { Big Wills Creek } \ldots \ldots \ldots \ldots\end{array}$ & $\begin{array}{l}\text { Mouth of Creek...... } \\
\text { Mouth of Creek..... } \\
\text { Above Little Wills }\end{array}$ & $\begin{array}{r}165 \\
34 \\
354\end{array}$ & $\begin{array}{r}65 \\
14 \\
160\end{array}$ & 14.3 \\
\hline $\begin{array}{l}\text { Right } \\
\text { Left.. } \\
\text { Right } \\
\text { Left.. } \\
\text { Ieft.. } \\
\text { Right } \\
\text { Right| } \\
\text { Right } \\
\text { Right }\end{array}$ & 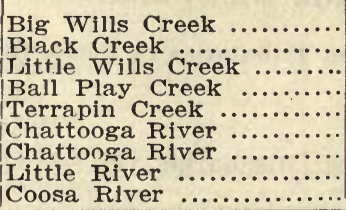 & $\begin{array}{l}\text { Above Wesson Mili. } \\
\text { Mouth of Creek....... } \\
\text { Mouth of Creek...... } \\
\text { Mouth of Creek...... } \\
\text { Mouth of Creek..... } \\
\text { Above Little River.. } \\
\text { Ala.-Ga. State Line } \\
\text { Mouth of River ..... } \\
\text { Ala.-Ga. State Line }\end{array}$ & $\begin{array}{r}249 \\
200 \\
59 \\
30 \\
33 \\
282 \\
394 \\
246 \\
280 \\
4340 \\
\end{array}$ & $\begin{array}{r}115 \\
107 \\
25 \\
14 \\
15 \\
130 \\
170 \\
121 \\
130 \\
2000\end{array}$ & $\begin{array}{r}1.4 \\
11.8 \\
15.4 \\
11.0 \\
11.8 \\
181.8\end{array}$ \\
\hline
\end{tabular}

NoTE-To find the net horsepower availahle at a shoal on one of these streams, near a point given, for low water 1900-1901, multiply the total fall of the shoal by the "net borsepower per foot fall" in this table for that point.

\section{WATER POWERS ON TRIBUTARIES OF COOSA RIVER IN} ALABAMA.

On the above named tributaries there are many important water powers, very few of which have been surveyed. The ar ove list giving the drainage area, the discharge for low season, I900-I9OI, and the corresponding net horse power per foot fall for each of the streams will be very useful in estimat- 
ing the horse power available on any shoal, the fall of which may hereafter be surveyed, by the owners, or by parties contemplating development.

Talladega Creek, in the vicinity of 'Taylor's Mill, has a fall of 73 feet in one mile, where it emerges from the Crystalline rocks. Taking the flow at Nottingham, we say that during the low water of 1900 and r 901 this 73 feet of fall would have produced 438 net horse power without storage. This 73 feet is probably the most precipitous shoal on the large creek, but above it for four or five miles the creek has a number of rapids and shoals that will admit of good development.

The head waters of this stream in the neighborhood of the pyrites mines in Clay county have high falls on them.

Choccolocco Creek is a very large and constant stream, and has many rapids where good powers could be developed by dams. During a season such as low water of 1900 or I90I a Io-foot dam near Jenifer would develop 86 net H. P. A rofoot dam at any point near the mouth of the creek would develop 140 net $H$. P. during the given season.

Big Wills Creek, at the old Wesson mill, two miles north of Attalla, has a good site for a 25-foot dam. The flow at this point on October 16, I901, was ro7 second-feet, which with a fall of 25 feet, will give 242 net H. P. The fall on other tributaries named has not been ascertained.

\section{COOSA RIVER SURVEY.}

The Coosa River has its beginning at the junction of the Etowah and Oastanaula Rivers, at Rome, Ga., a short distance west of the Alabama line.

From Rome down to Greensport, Ala., a distance of about I8o miles by river, navigation has been carried on for many years. The total fall in this section is only about 55 feet, and is so well distributed that it has not been necessary to construct locks at any point, though improvements have been made by the U. S. Government in the way of deepening channels, blasting out reefs, and building wing dams, etc.

This part of the river will, therefore, not be considered as having any water power value.

Below Greensport, Ala., the river has a large amount of fall, and although it is proposed to make the whole distance navigable by the construction of locks, there are many fine water power propositions which can be developed in connection with 
the river improvements without interfering with navigation.

A complete survey has been made of this portion of the river by the U. S. engineers, and a system of locks planned.

The profile herein presented is reproduced from that survey, and shows in addition to the river profile the location of the proposed locks, and the lift of each.

It will be seen that the total distance between Greensport and Wetumpka, Ala., is 142 miles, and the number of locks proposed, $3 \mathrm{I}$, varying in lift from 5.83 feet to 15.0 feet. Of these only three have been, completed; Nos. I, 2, and 3. No. 4 is in process of construction.

The following table shows the lift or fall at each lock, the discharge of river in cubic feet per second, for the minimum low stage of water in 1897 and in 1900 , and the equivalent net horse power for the fall shown.

The minimum low water is based on the exceptionally low stages occurring in 1896 and 1897 , which represents the lowest stage of which there is any record; while the minimum for the year 1900 represents lowest water for average years.

In estimating the amount of horse power that will be available for use, it will be necessary to deduct the amount of water which will be necessary for lockage. This will depend upon the amount of traffic on the river, but will probably in no case amount to more than ten per cent. of the river discharge.

At most of these locks, and proposed locks, reservations have been made by the original owners of the river front of the privilege of utilizing for power the water not needed for lockage. By constructing a plant at the opposite end of the Government dam from the lock, the surplus water can be used for power without interfering with navigation. Such powers will be very valuable for running cotton mills, as the cotton furrows will run up to the front door of the factory, and water transportation will take the goods from the back door. Mobile, at the mouth of the river, is only a short distance from the proposed Isthmian canal. 
9. 'TABLE OF DISCHARGE, AND NET H. P. AT THE 31 LOCKS AND PROPOSED LOCKS ON THE COOSA RIVER AT LOWEST WATER OF 1897 AND 1900.

[80 \% of Theoretical H. P.]

\begin{tabular}{|c|c|c|c|c|c|c|c|}
\hline \multirow{2}{*}{ 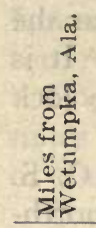 } & \multirow[b]{2}{*}{ 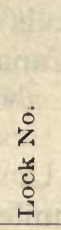 } & \multirow[b]{2}{*}{ 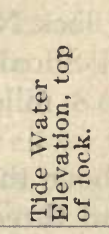 } & \multirow[b]{2}{*}{ 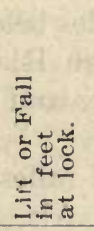 } & \multicolumn{2}{|c|}{$\begin{array}{c}\text { Minimum Low } \\
\text { Water, } 1897 .\end{array}$} & \multicolumn{2}{|c|}{$\begin{array}{c}\text { Minimum Low } \\
\text { Water, } 1900 .\end{array}$} \\
\hline & & & & 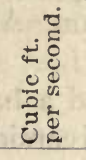 & $\begin{array}{l}\dot{2} \\
\text { I } \\
\ddot{0} \\
z\end{array}$ & 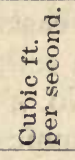 & 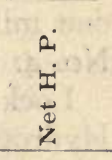 \\
\hline 141.5 & 1 & 521.30 & 5.33 & 1,320 & 640 & 2,700 & 1,308 \\
\hline 138.5 & 2 & 515.97 & 5.57 & 1,320 & 668 & 2,700 & 1,367 \\
\hline 137.0 & 3 & 510.40 & 12.00 & 1,320 & 1,440 & 2,700 & 2,945 \\
\hline 116.2 & 4 & 492.30 & 10.00 & 1,350 & 1,227 & 2,760 & 2,510 \\
\hline 105.8 & 5 & 482.30 & 12.00 & 1,350 & 1,472 & 2,760 & 3,012 \\
\hline 92.0 & 6 & 455.32 & 10.00 & 1,440 & 1,310 & 2,940 & 2,673 \\
\hline 88.3 & 7 & 445.32 & 10.00 & 1,450 & 1,317 & 2,960 & 2,690 \\
\hline 81.3 & 8 & 435.32 & 12.00 & 1,490 & 1,625 & 3,040 & 3,317 \\
\hline 56.2 & 9 & 420.00 & 8.00 & 1,580 & 1,149 & 3,220 & 2,342 \\
\hline 53.5 & 10 & 412.00 & 12.00 & 1,585 & 1,728 & 3,230 & 3,523 \\
\hline 46.7 & 11 & 399.64 & 10.00 & 1,585 & 1,440 & 3,230 & 2,936 \\
\hline 44.9 & 12 & 389.64 & 10.00 & 1,600 & 1,454 & 3,260 & 2,964 \\
\hline 43.0 & 13 & 379.64 & 12.00 & 1,600 & 1,745 & 3,260 & 3,557 \\
\hline 41.9 & 14 & 367.64 & 12.00 & 1,600 & 1,745 & 3,260 & 3,557 \\
\hline 40.2 & 15 & 355.64 & 10.00 & 1,605 & 1,460 & 3,270 & 2,973 \\
\hline 37.5 & 16 & 345.64 & 14.00 & 1,605 & 2,044 & 3,270 & 4,162 \\
\hline 36.1 & 17 & 331.64 & 15.00 & 1,605 & 2,190 & 3,270 & 4,460 \\
\hline 34.8 & 18 & 316.64 & 13.00 & 1,610 & 1,903 & 3,280 & 3,877 \\
\hline 33.8 & 19 & 303.64 & 12.00 & 1,610 & 1,757 & 3,280 & 3,578 \\
\hline 31.5 & 20 & 291.64 & 10.00 & 1,610 & 1,464 & 3,280 & 2,982 \\
\hline 25.5 & 21 & 281.33 & 10.00 & 1,700 & 1,545 & 3,460 & 3,145 \\
\hline 21.4 & 22 & 270.80 & 12.00 & 1,700 & 1,854 & 3,460 & 3,774 \\
\hline 18.5 & 23 & 258.80 & 14.00 & 1,710 & 2,175 & 3,480 & 4,430 \\
\hline 16.3 & 24 & 244.80 & 10.00 & 1,710 & 1,554 & 3,480 & 3,164 \\
\hline 12.9 & 25 & 234.80 & 10.00 & 1,710 & 1,554 & 3,480 & 3,164 \\
\hline 11.7 & 26 & 224.80 & 12.00 & 1,720 & 1,877 & 3,500 & 3,818 \\
\hline 8.8 & 27 & 212.80 & 14.00 & 1,720 & 2,190 & 3,500 & 4,455 \\
\hline 7.4 & 28 & 198.80 & 12.00 & 17,20 & 1,877 & 3,500 & 3,818 \\
\hline 4.6 & 29 & 186.37 & 8.00 & 1,740 & 1,266 & 3,540 & 2,574 \\
\hline 2.0 & 30 & 178.37 & 10.00 & 1,740 & 1,582 & 3,540 & 3,218 \\
\hline 0.0 & 31 & 168.37 & 14.00 & 1,740 & 2,215 & 3,540 & 4,505 \\
\hline Tota & & P... & & & 49,467 & & 100.798 \\
\hline
\end{tabular}

Locks and proposed locks on Coosa River are located as follows :

Lock No. I is one mile south of Greensport, Ala., and five miles north of Singleton, which is a station on the East \& West Railroad of Alabama. Lock No. I is three miles above lock No. 2. 
Lock No. 2 is one and a half miles above lock No. 3 , and is located at the head of Woods Island, and two miles northeast of Singleton, Ala., which is a station on the East \& West Railroad. This lock is situated at the head of Ten Island Shoal Canal.

Lock No. 3 is one and a half miles below lock No. 2, near the foot of Woods Island, and on Ten Island Shoal Canal. It is one mile east of Singleton, Ala., and 20.8 miles above lock No. 4.

Lock No. 4 is three and a half miles above the U. S. G. S. Hydrographic Station, Riverside, Ala., and three miles northwest of Lincoln, Ala. Lincoln and Riverside are on the Georgia Pacific division of the Southern Railway. Lock No. 4 has a lift of 12 feet, and is three-quarters of a mile below Denson's Island, and ten miles above proposed lock No. 5 .

Proposed lock No. 5 is to be at the head of Ogletree Island, one mile above the mouth of Choccolocco Creek, and five miles northeast of Hamilton, on the Talladega \& Coosa Valley Railroad. Has a lift of ten feet.

Proposed lock No. 6 is to be located one-fourth of a mile above the mouth of Upper Clear Creek, one and a half miles above Grissom's Ferry, and nine miles north-east of Vincent, which is a station on the Columbus \& Western division of the Central of Georgia Railroad.

Proposed lock No. 7 is to be located two miles above Kelly Creek, and five and a half miles north-east of Vincent, Ala.

Proposed lock No. 8 is to be located at Myer's Ferry, at the mouth of Lower Clear Creek, six miles east of Harpersville, and three miles north-east of Creswell, which is a station on the Columbus \& Western division of the Central of Georgia Railroad.

Proposed lock No. 9 is to be located at the mouth of Kelly Branch, at Fort Williams Shoals. It is to be thirteen and a half miles east of Columbiana, Ala., and eight miles east of Shelbv, Ala.

Lock No. to is to be located a half mile above Peckerwood Creek, at the foot of Peckerwood Shoals, and is eight miles east of Shelby, Ala., and two miles west of Talladega Springs, Ala.

Lock No. I I is to be located at the foot of Weduska Shoals, immediately above the narrows, two miles above Waxahatchee Creek, and six miles south-east of Shelby, Ala., which is a station on the Shelby Iron Works Railroad, connecting with the E. T., V. \& G. R. R. at Columbiana, Ala. 
Lock No. 12 is to be located I. 8 miles below lock No. I I, immediately below the mouth of Waxahatchee Creek, and eight miles south-east of Shelby, Ala.

Lock No. 13 is to be located I.9 miles below lock No. 12, at a place known as Devil's Race, three miles above the mouth of Lower Yellow Leaf Creek, and sixteen miles north-east of Clanton, Ala., on the L. \& N. R. R.

Lock No. I4 is to be located one mile below lock No. I3, two miles above Yellow Leaf Creek, and fourteen miles north-east of Clanton, Ala.

Lock No. ${ }_{5} 5$ is to be located I.7 miles below lock No. I4, three-tenths of a mile above Lower Yellow Leaf Creek, and twelve miles north-east of Clanton, Ala., on the L. \& N. R. R.

Lock No. 16 is to be located 2.7 miles below lock No. 15, at Butting Ram Shoals, which is eleven miles north-east of Clanton, Ala.

Lock No. 17 is to be located $\mathrm{r} .4$ miles below lock No. I6, and is ten and a half miles north-east of Clanton, Ala.

Lock No. 18 is to be located I.3 miles below lock No. 17, and eleven miles east of Clanton, Ala.

Lock No. 19 is to be located one mile below lock No. I8, about eleven miles east of Clanton, Ala.

Lock No. 20, 31.5 miles above Wetumpka, one-fourth mile above Zimmerman's Ferry, I.2 miles above the mouth of Hatchet Creek.

Lock No. 21, 25.5 miles above Wetumpka, I.6 miles below mouth of Blue Creek, 7 miles east of Cooper, Ala., on L. \& N. R. R.

Lock No. 22, 2I.4 miles above Wetumpka, three-fourths of a mile below the mouth of Proctors Creek, and r.I miles above the mouth of Pinchoulee Creek, and 7 miles east of Verbena, Ala., on the L. \& N. R. R.

Lock No. 23, I8.5 miles from Wetumpka, r.5 miles below the mouth of Pinchoulee Creek.

Lock No. 24, I6. miles above Wetumpka, 0.4 miles below the mouth of Welcree Creek, and seven and a half miles east of Mountain Creek Station, on the L. \& N. R. R.

Lock No. 25, 12.9 miles above Wetumpka, O.I miles above the mouth of Shoal Creek, and about 8 miles east of Wadsworth, Ala., on the L. \& N. R. R.

Lock No. 26, Ir.7 miles above Wetumpka, at Staircase Falls, just above the mouth of Wewoka Creek. 
Lock No. 27, 8.8 miles above Wetumpka, 0.6 miles above the mouth of Sofkahatchee Creek, and about nine miles east of Deatsville, Ala., on the L. \& N. R. R.

Lock No. 28, 7.4 miles above Wetumpka.

Lock No. 29, 4.6 miles above Wetumpka.

Lock No. 30, 2 miles above Wetumpka.

Lock No. 31, at Wetumpka, Ala. 


\section{CHAPTER IV.}

1. CAHABA RIVER AT CENTERVILLE. ALABAMA.

Centerville Station, on Cahaba River, is at the Bibb county highway bridge, one-fourth of a mile west of the court house at Centerville, Ala. The bridge is a single span iron through bridge. The length of the span is about 175 feet. The floor of the bridge is $4 \mathrm{I} 1 / 2$ feet above low water, and the stream is $\mathrm{I} 30$ feet wide at low water.

The initial point of sounding is at the end of the iron bridge, left bank, down stream. The gage is a wire gage, with rod fastened to the outside of down stream guard rail, and graded to feet and tenths. The gage pulley is at Station Ioo. Bench mark No. I, down stream end of top of iron crossbeam under the bridge floor at Station IOO, from initial point is 42.85 above gage datum.

Bench mark No. 2, top of bottom flange of same crossbeam, directly under B. M. No. I, is $4 \mathrm{I} .40$ above datum of gage.

Banks are high, but overflow at time of high water.

The section is swift, and tolerably uniform, and the bottom appears to be rock.

The river observer is Mr. S. D. Hall, a merchant, who lives. about a quarter of a mile from the gage.

The following discharge measurements have been made on Cahaba River at Centerville, Ala. : 1901.

April 25-Hydrographer, J. R. Hall; gage height, 5.50; discharge, 1,925 second-feet.

Aug. 7-Hydrographer, K. T. Thomas; gage height, 1.30; discharge, 399 second feet. 1902 .

Jan. 25-Hydrographer, K. T. Thomas; gage height, 5.15; discharge, 1,707 second-feet.

Daily gage height of Cahaba River at Centerville, Ala, for 1901.

\begin{tabular}{|c|c|c|c|c|c|c|c|c|c|c|c|}
\hline Day & Aug. & Sept. & Oet. & Nov. & Dec & Day & Aug. & Sept. & Oct. & Nov. & Dec \\
\hline 1.. & & 2.20 & 2.10 & 1.20 & 1.4 & 17 & 7.70 & 2.10 & 1.30 & 1.30 & 12.3 \\
\hline & & & 2.00 & & 1.3 & & 8.10 & & & & 4.4 \\
\hline $3 .$. & & 1.90 & 1.80 & 1.20 & 1.6 & 19 & 9.10 & & & & 3. \\
\hline $4 .$. & & 1.8 & 1.40 & 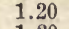 & 1.9 & 20 & 10.60 & & & 1.1 & \\
\hline $5 .$. & & 1.6 & 2.30 & & 1.8 & 21 & 14.7 & & & & 2.9 \\
\hline $6 .$. & & 1.5 & 4.60 & 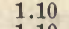 & 1.6 & 22 & 10.30 & 1. & & & 2. \\
\hline 7.. & 1.30 & 1.4 & 3.1 & & 1.7 & 23. & 7.90 & 1.8 & & 1.60 & 2.5 \\
\hline $8 .$. & 1.30 & 1.4 & 2.60 & 1 & 1.6 & 24 & 5.60 & 1. & & & \\
\hline & 1.20 & 1. & 2.3 & & 1.7 & 25 & 4.8 & & & 1.5 & 2. \\
\hline $10 .$. & 1.20 & 1.40 & 1.90 & 1.20 & 2.1 & 26 & 4.10 & 1.4 & 1. & & 2. \\
\hline 11. & 1.20 & & 1.6 & & 2.0 & & & 1.4 & 1. & 1.50 & 2. \\
\hline 12. & 1.20 & 1.3 & 1.40 & 1.3 & 1.8 & 98. & 3.40 & 1.4 & 1. & & 6. \\
\hline $13 \ldots \ldots$ & 1.30 & 1.3 & 1.4 & 1.4 & 1.9 & & 3.00 & 2.6 & 1.3 & 1.40 & 24.0 \\
\hline $14 \ldots \ldots$ & 1.50 & 2.6 & 1.40 & 1.3 & 2.9 & & 2.80 & 2.20 & & 1.40 & 24. \\
\hline & 2.10 & 2.4 & 1.30 & 1.30 & 19.0 & $31 .$. & 2.50 & & 1.30 & & 21.0 \\
\hline $16 \ldots \ldots$ & 7.90 & 2.00 & 1.30 & 1.30 & 15. & & & & & & \\
\hline
\end{tabular}


Rating table for Cadiaba River at Centerville, Alabama, for year 1901.

\begin{tabular}{|c|c|c|c|c|c|c|c|}
\hline & 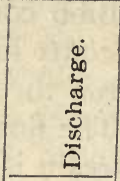 & 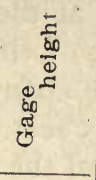 & 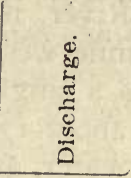 & 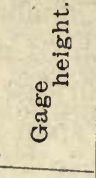 & 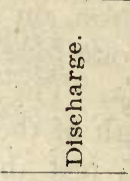 & 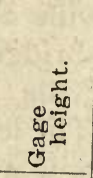 & 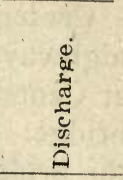 \\
\hline Feet. & Second $f t$. & Feet. & Second ft. & Feet. & second ft. & Feet. & second ft. \\
\hline 1.1 & 326 & 4.9 & 1,694 & 8.7 & 3,062 & 12.5 & 4,430 \\
\hline 1.2 & 362 & 5.0 & 1,730 & 8.8 & 3,098 & 12.6 & 4,466 \\
\hline 1.3 & 398 & 5.1 & 1,766 & 8.9 & 3,134 & 12.7 & 4,502 \\
\hline 1.4 & 434 & 5.2 & 1,802 & 9.0 & 3,170 & 12.8 & 4,538 \\
\hline 1.5 & 170 & 5.3 & 1,838 & 9.1 & 3,206 & 12.9 & 4,574 \\
\hline 1.6 & 506 & 5.4 & 1,874 & 9.2 & 3,242 & 13.0 & 4,610 \\
\hline 1.7 & 542 & 5.5 & 1,910 & 9.3 & 3,278 & 13.1 & 4,646 \\
\hline 1.8 & 578 & 5.6 & 1,946 & 9.4 & 3,314 & 13.2 & 4,682 \\
\hline 1.9 & 614 & 5.7 & 1,982 & 9.5 & 3,350 & 13.3 & 4,718 \\
\hline 2.0 & 650 & 5.8 & 2,018 & 9.6 & 3,386 & 13.4 & 4,754 \\
\hline 2.1 & 686 & 5.9 & 2,054 & 9.7 & 3,422 & 13.5 & 4,790 \\
\hline 2.2 & 722 & $6: 0$ & 2,090 & 9.8 & 3,458 & 13.6 & 4,826 \\
\hline 2.3 & 758 & 6.1 & 2,126 & 9.9 & 3,494 & 13.7 & 4,862 \\
\hline 2.4 & 794 & 6.2 & 2,162 & 10.0 & 3,530 & 13.8 & 4,898 \\
\hline 2.5 & 830 & 6.3 & 2,198 & 10.1 & 3,566 & 13.9 & 4,934 \\
\hline 2.6 & 866 & 6.4 & 2,234 & 10.2 & 3,602 & 14.0 & 4,970 \\
\hline 2.7 & 902 & 6.5 & 2,270 & 10.3 & 3,638 & 14.1 & 5,006 \\
\hline 2.8 & 938 & 6.6 & 2,306 & 10.4 & 3,674 & 14.2 & 5,042 \\
\hline 2.9 & 974 & 6.7 & 2,342 & 10.5 & 3,710 & 14.3 & 5,078 \\
\hline 3.0 & 1,010 & 6.8 & 2,378 & 10.6 & 3,746 & 14.4 & 5,114 \\
\hline 3.1 & 1,046 & 6.9 & 2,414 & 10.7 & 3,782 & 14.5 & 5,150 \\
\hline 3.2 & 1,082 & 7.0 & 2,450 & 10.8 & 3,818 & 14.6 & 5,186 \\
\hline 3.3 & 1,118 & 7.1 & 2,486 & 10.9 & 3,856 & 14.7 & 5,222 \\
\hline 3.4 & 1,154 & 7.2 & 2,522 & 11.0 & 3,890 & 14.8 & 5,258 \\
\hline 3.5 & 1,190 & 7.3 & 2,558 & 11.1 & 3,926 & 14.9 & 5,294 \\
\hline 3.6 & 1,226 & 7.4 & 2,594 & 11.2 & 3,962 & 15.0 & 5,330 \\
\hline 3.7 & 1,262 & 7.5 & 2,630 & 11.3 & 3,998 & 15.1 & 5,366 \\
\hline 3.8 & 1,298 & 7.6 & 2,666 & 11.4 & 4,034 & 15.2 & 5,402 \\
\hline 3.9 & 1,334 & 7.7 & 2,702 & 11.5 & 4,070 & 15.3 & 5,438 \\
\hline 4.0 & 1,370 & 7.8 & 2,738 & 11.6 & 4,106 & 15.4 & $5, \div 74$ \\
\hline 4.1 & 1,406 & 7.9 & 2,774 & 11.7 & 4,142 & 15.5 & 5,510 \\
\hline 4.2 & 1,442 & 8.0 & 2,810 & 11.8 & 4,178 & 15.6 & 5,546 \\
\hline 4.3 & 1,478 & 8.1 & 2,846 & 11.9 & 4,214 & 15.7 & 5,582 \\
\hline 4.4 & 1,514 & 8.2 & 2,882 & 12.0 & 4,250 & 15.8 & 5,618 \\
\hline 4.5 & 1,550 & 8.3 & 2,918 & 12.1 & 4,286 & 15.9 & 5,654 \\
\hline 4.6 & 1,586 & 8.4 & 2,954 & 12.2 & 4,322 & 16.0 & 5,690 \\
\hline 4.7 & 1,622 & 8.5 & 2,996 & 12.3 & 4,358 & & \\
\hline
\end{tabular}

NotE.-This table applied to the foregoing "Daily gage heights" gives the cubic feet per second flowing in the river on each date for which the gage height is given. 
Minimum monthly discharge of Cahaba River at Centerville, Ala.. with corresponding net horsepower per foot of fall on a water wheel realizing 80 per cent. of the theoretical power.

\begin{tabular}{|c|c|c|c|}
\hline & \multicolumn{3}{|c|}{1901.} \\
\hline & 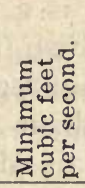 & 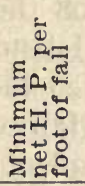 & 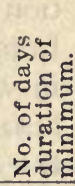 \\
\hline August & 362 & 33 & 4 \\
\hline September $\quad \ldots \ldots \ldots \ldots \ldots \ldots \ldots \ldots$ & 398 & 36 & 3 \\
\hline October $\ldots \ldots \ldots \ldots \ldots \ldots \ldots \ldots \ldots \ldots \ldots$ & 362 & 33 & 5 \\
\hline November $\quad \ldots \ldots \ldots \ldots \ldots \ldots \ldots \ldots$ & 326 & 30 & 3 \\
\hline December...$\ldots \ldots \ldots \ldots \ldots \ldots$ & 398 & 36 & 1 \\
\hline
\end{tabular}

NoтE.-To find the minimum net horse power available at a shoa? on this stream, near this station, for any month, multiply the total fall of the shoal by the "net H. P. per foot of fall" in this table for that month.

2. SURVEY OF CAHABA RIVER, ALABAMA.

The Cahaba River rises near Birmingham, Alabama, and flowing in a southerly direction, enters the Alabama Ri:ter at a point just below Selma, Alabama.

From the published notes of a survey made by the Corps of Engineers, U. S. A., a profile has been made beginning at the southwestern boundary of Shelby county, and running down the river a distance of I Io miles to its mouth, in which distance there is a fall of 227 feet.

A United States Geological Survey hydrographic station has been established at Centerville, Ala,. and measurements of discharge have also been made at Sydenton, near Birmingham, but the measurements and observations have not extended over a sufficient length of time to give accurate estimates of discharge at all seasons. The best that can be done at present is to form an estimate of the river discharge for ordinary low stage of water at Centerville, which is 344 second-feet, and add to or subtract from this amount for other points on the river. From measurements made on the same day at Centerville and at Harrell, near the mouth of the river, we estimate that the flow at Centerville is about one-third of that at the mouth of the river. 
On the accompanying profile the stations are one mile apart, and are numbered from zero at the mouth of the river, up to I Io at the Shelby county line. In the following description of powers that can be developed these mile stations will be referred to as stations :

Power No. I.-From the head of "Half Mile Rapids" at Station 108 there is a succession of shoals known as Half Mile, Long Island, Fish Trap, Ford, Reach, and Dry Creek Shoals, in which the aggregate fall is 30 feet in $2 \frac{1}{4}$ miles. There is also a fall of about 4 feet from the Shelby County line down to the head of Half Mile Shoal, making a total fall of 34 feet in four miles. This can be developed either by building a dam 34 feet high at the mouth of Dry Creek and backing the water to the Shelby County line, or by building a low dam near the head of the shoals, and a canal from it to a point opposite the mouth of Dry Creek. Such a development will give about 500 net horse-power, with an $80 \%$ turbine at ordinary low season. This power would be near Blocton, Ala.

Power No. 2.-By building a I.5-foot dam at the head of "Baily Reach Rapids," near Station IOr, and near the mouth of Big Ugly Creek, to back the water to the mouth of Persimmon branch near Station IO4, and constructing from this point a canal along the river bank about four miles long, to a point opposite Station 97, at the mouth of Little Cahaba River, a practical head of 54 feet can be developed. This allows 8 feet for storage and grade, as the total fall is 62 feet. A 54 -foot fall would produce about 800 net $\mathrm{H}$. P.

The same power can be developed by building a high dam, lower down the river, and having the canal shorter. Or, the power can be divided into two separate powers. This power site is between River Bend and Cadle, in Bibb County.

Power No. 3.-From the mouth of Little Cahaba down to Station $881 / 2$ at the top of Centerville Shoals, there is a fall of ten feet in $81 / 2$ miles, and from the top of Centerville Shoals down to the foot of Centerville Shoals at Centerville, there is a fall of 13.6 feet in about $13 / 4$ miles. This power can be developed by a ten-foot dam at top of Centerville Shoals, and a canal from there to Centerville $\mathrm{I}^{\mathrm{I} / 2}$ miles long. Allowing 2.5 feet for storage and canal grade, a head of 21 feet can be obtained which will give 650 net $\mathrm{H}$. P.

It is probable that a much better method of development will be to erect a dam at Centerville 23.6 feet high to back the 
water to the mouth of Little Cahaba. This will produce 732 net H. P., with storage. The incidental storage of such a dam would add largely to the amount and efficiency of the power. A plant running only 12 hours per day, and storing the water at night, could utilize $\mathrm{I}, 440$ net $\mathrm{H}$. P.

This power site is at Centerville. Alabama, on the M. \& O. Railroad.

Power No. 4.-A I6-foot dam can be built at Shoal No. 9, Station $69 \frac{1}{2}$, in Perry County, just below the Bibb Connty line. This dam would back the water for 12 miles to Shoal No. $2,4 \frac{\mathrm{T} / 2}{2}$ miles below Centerville. A I 6 -foot head will produce 670 $\mathrm{H}$. P. without storage, or $1,340 \mathrm{H}$. P. by storing the waier at night, and running only twelve hours per day. This dan site is about 17 miles below Centerville by river.

Power No. 5.-A i 5-foot dam at "Blocks Cut-off," near Station 55, will back the water ten miles to the mouth of Taylor's Creek, and will produce 750 continuous, or 1,500 twelve-hour horse power.

Power No. 6.-At Shoal No. 24, Station 50, there is a fall of 9 feet in less than half a mile. A I4-foot dam at foot of this shoal, or a 5 -foot dam at its head, and a short canal will develop a head-of 14 feet and realize 720 continuous, or $\mathrm{r}, 400$ twelvehour H. P.

This site is just above Burras Island, 8 or to miles northeast of Marion, Ala.

Power No. 7.-From Burras Island to Fikes Ferry there is a fall of 22 feet in a distance of 7 miles, 20 feet of which could probably be utilized by a dam at Fikes Ferry, producing I, IOO continuous, or 2,200 twelve-hour H. P. Fikes Ferry is near Marion, Ala.

In making the above statement of powers that can be c.eveloped, it has been assumed that there are suitable banks for dam sites. The system proposed, or some other system approximating to it, would not interfere with navigation improvements, as locks could be constructed at the dams. 
CHAPTER V.

\section{BLACK WARRIOR RIVER AND TRIBUTARIES}

\section{i. TUSCALOOSA STATION ON BLACK WARRIOR RIVER,}

This gage was placed in position by the United Statcs Cor's of Engineers in I888. It is about three-fourths of a mile from the business center of Tuscaloosa, Alabama, and is reached by passing down Bridge street to the river, thence down the east bank $\mathrm{I}, 800$ feet to the gage. It consists of an inclinci timber, $2 \times 6$ inches, supported on posts and graduated by means of notches placed I foot vertically apart. The observer is $\mathrm{W}$. S. Wyman, Jr., Tuscaloosa, Alabama. Mr. Wyman is observer for the Corps of Engineers, and has been kind enough to send weekly reports to this office. Observations are taken daily at 7 A. M. The area draining past this point is 4,900 square miles.

The bench marks are fixed, one on a willow Io feet west of gage, 97.84 feet above Mobile datum, the other on a small hackberry 30 feet south of the upper end of the gage and $\mathrm{r} 39.36$ feet above Mobile datum. The current here is rather sluggish, being almost imperceptible at low stages. Both banks are of earth, and subject to overflow. Observations of gage heights have been obtained through the courtesy of Mr. R. C. McCalla, Jr., of the United States Engineers in charge of the Black Warricr River, from the time the gage was established until December 31, I896. A measurement made by Mr. McCalla September I4, I896, showed a gage height of -0.60 foot area, I,022 square feet, mean velocity 0.16 , discharge 164 second-feet.

The following list of measurements at the same place has been furnished by Mr. Horace Harding, C. E., United States assistant enginee, 2016 Quinlan avenue, Birmingham, Alabama. Velocities were obtained by means of rod floats reaching from the water surface to near the bottom. The highest flood occurred on April 8, 1892. The gage height was 62.5 , the sectional area 33,600 square feet, and the estimated mean velocity 4.5 feet per second. This gave a discharge of I5I,200 cubic feet per second. From this estimate and the following list of measurements a curve has been plotted and a rating table constructed, and this rating table applied to all gage heights observed. The estimates of orscharge thus obtained are shown in diagrammatic. form in Plate V. The highest discharges are merely approximations, but the discharges shown by the diagrams serve as a basis for comparison of the state of the river during the various years. 
List of Discharge Measurements made on Black Warrior River at Tuscaloosa, Alabama.

\begin{tabular}{|c|c|c|c|c|}
\hline No. & Date. & $\begin{array}{l}\text { Gage } \\
\text { height. }\end{array}$ & Discharge. & Remarks. \\
\hline & 1895. & Feet. & Second-feet. & \\
\hline 1 & Dec. 17 & 1.10 & 617 & Stationary. \\
\hline 2 & Dec. 21 & 2.61 & 1,344 & Do. \\
\hline 3 & Dec. 24 & 3.60 & 1,733 & Rising slowly. \\
\hline & 1896. & & & \\
\hline 4 & Jan. 30 & 9.99 & 5,073 & Falling 0.05 per hour. \\
\hline 5 & Jan. 31 & 8.65 & 4,363 & Do. \\
\hline 6 & Feb. 26 & 8.25 & 4,360 & Falling 0.01 per hour. \\
\hline 7 & Feb. 28 & 7.27 & 3,657 & Falling 0.02 per hour. \\
\hline 8 & Feb. 29 & 6.92 & 3,522 & Stationary. \\
\hline 9 & Mar. 2 & 7.67 & 4,211 & Do. \\
\hline 10 & Mar. 3 & 7.28 & 3,632 & Falling 0.03 per hour. \\
\hline 11) & Mar. 6 & 6.94 & 4,558 & Rising 0.15 per hour. \\
\hline 12 & Mar. 24 & 24.85 & 13,550 & Falling 0.12 per hour. \\
\hline 13 & Apr. 10 & 9.71 & 5,331 & Falling. \\
\hline 14 & Apr. 11 & 8.89 & 4,755 & Do. \\
\hline 15 & Apr. 14 & 8.25 & 4,675 & Rising. \\
\hline 16 & Apr. 20 & 7.55 & 3,862 & Falling. \\
\hline 17 & Apr. 21 & 6.65 & 3,388 & Do. \\
\hline 18 & Apr. 22 & 5.96 & 2,940 & Do. \\
\hline 19 & Apr. 23 & 5.46 & 2,704 & Do. \\
\hline 20 & Apr. 24 & 5.88 & 3,158 & Rising. \\
\hline 21 & Apr. 27 & 5.68 & 3,049 & Do. \\
\hline
\end{tabular}


Daily gage height of Black Warrior River at Tuscaloosa, Ala., for 1889.

\begin{tabular}{|c|c|c|c|c|c|c|c|c|c|c|c|c|}
\hline Day & Jan. & Feb. & Mar. & April & May & Jun. & July & Aug. & Sept. & Oct. & Nov. & Dec \\
\hline & & 3.50 & 18.80 & 8.50 & 8.80 & 2.30 & 2.40 & 3.70 & 0.75 & 2.40 & 0.90 & .00 \\
\hline & & 20.50 & $16.50 \mid$ & 9.80 & $\mid$\begin{tabular}{|c|}
14.50 \\
12
\end{tabular} & $\begin{array}{r}2.90 \\
.75\end{array}$ & 3.10 & 3.60 & 7.30 & 1.95 & 1.05 & \\
\hline & & & 25.00 & 10.30 & \begin{tabular}{|l|}
13.20 \\
11
\end{tabular} & 2.75 & 4.40 & 3.00 & 6.40 & 1.55 & 1.85 & \\
\hline & 16.00 & $\begin{array}{l}14.80 \\
13.00\end{array}$ & $\mid \begin{array}{l}31.00 \\
29\end{array}$ & 10.50 & 8.50 & 2.00 & 5.10 & $2.9 \mathrm{a}$ & 5.30 & 1.30 & 1.90 & \\
\hline & .00 & 11.50 & $26.50 \mid$ & $\begin{array}{r}10.00 \\
9.50\end{array}$ & $\begin{array}{l}0.00 \\
7.00 \\
\end{array}$ & 2.10 & $\begin{array}{l}0.20 \\
6.85\end{array}$ & 3.70 & $\begin{array}{l}0.20 \\
9.30\end{array}$ & $\begin{array}{l}1.10 \\
1.05\end{array}$ & $\begin{array}{l}3.89 \\
3.65\end{array}$ & 4.50 \\
\hline & 33.50 & 10.40 & 23.00 & 8.40 & 5.80 & 1.90 & 6.45 & $\begin{array}{l}3.45 \\
3.45\end{array}$ & \begin{tabular}{|}
74.90 \\
\end{tabular} & $\begin{array}{r}1.00 \\
.90\end{array}$ & $\begin{array}{l}0.00 \\
3.30\end{array}$ & $\begin{array}{l}4.00 \\
4.00\end{array}$ \\
\hline & & 9.30 & 20.10 & 7.30 & 4.60 & 1.70 & 5.70 & 2.95 & 19.00 & .70 & 3.00 & \\
\hline & 26.80 & 8.30 & 17.50 & 6.80 & 4.30 & 2.00 & 4.75 & 2.60 & 14.00 & .50 & 2.75 & \\
\hline & 28.44 & 8.00 & $15.00 \mid$ & 6.40 & 4.00 & 2. & 3.85 & 2.00 & 9.90 & .40 & 2.55 & \\
\hline & 29 & 7.80 & 12.80 & 6.30 & 3.80 & 2.85 & 3.05 & 1.75 & 5.50 & .30 & 3.65 & \\
\hline & & 7.60 & 11.00 & 6.00 & 3.20 & 4.20 & 2.85 & 1.55 & 5.25 & .30 & 4.30 & \\
\hline & 22.50 & 7.30 & 10.00 & 5.60 & 3.00 & 3.40 & 2.45 & 3.85 & 4.15 & .20 & 4.15 & \\
\hline & 19.00 & 7.00 & \begin{tabular}{|}
9.20 \\
\end{tabular} & 5.50 & 3.10 & 4.00 & 2.15 & 3.60 & 3.30 & .15 & & \\
\hline & 16. & 7.30 & 8.80 & 8.00 & 3.20 & 3.45 & 1.95 & 3.35 & 2.70 & .15 & & \\
\hline & 13.60 & 27.50 & 8.10 & 16.80 & 3.00 & 3.25 & 4.20 & 3.15 & 2.25 & .15 & 4. & \\
\hline & & 49.00 & 7.20 & 16.70 & 2.80 & 3.05 & 7.35 & 3.80 & 2.00 & .10 & 5.20 & \\
\hline & 40. & 56.40 & 7.10 & 14.00 & $? .50$ & 2.85 & 11.35 & 3.50 & 1.00 & .10 & & \\
\hline & 38.50 & 56.60 & 11.00 & 12.00 & 2.35 & 2.65 & 11.50 & 3.20 & 1.40 & .05 & & \\
\hline & 34. & 53.00 & 12.50 & 10.00 & 2.25 & 2.45 & 8.40 & 2.90 & 1.30 & .05 & 16. & \\
\hline & 30. & 47.00 & 12.30 & \begin{tabular}{|l|}
8.90 \\
\end{tabular} & 2.05 & 2.25 & 4.60 & 2.40 & 1.20 & .05 & 14. & \\
\hline & 28. & 41.5 & 11.80 & 8.0 & 1.85 & 3.25 & 4.55 & 1.90 & 1.10 & .601 & 11. & \\
\hline & 26. & & 10.80 & 7.0 & 1.65 & 3.45 & 4.50 & 1.50 & 1.00 & .10 & & \\
\hline & & & 10.00 & 6.4 ㄴ & 1.50 & 3.20 & 3.70 & 1.20 & 1.00 & .30 & & \\
\hline & & 28.50 & 9.80 & 6.30 & 1.30 & 3.00 & 3.15 & .90 & .95 & .15 & 6.6 & \\
\hline & & 26. & 11.20 & 6.8 & 1.25 & 2.40 & 3.10 & .75 & 2.25 & .10 & 6.30 & \\
\hline & 28. & 23.50 & 11.80 & 6.50 & 1.15 & 1.95 & 4.55 & 1.00 & 3.70 & .35 & 6.10 & \\
\hline & 33. & 21.40 & 12.30 & 6.2 & 1.05 & 1.55 & $4.0 \mathrm{~b}$ & .95 & 3.50 & .35 & 5.9 & 1. \\
\hline & & & 11.00 & 5.8 & .95 & 1.25 & 4.00 & .90 & 3.25 & .40 & 5.80 & 1. \\
\hline & & & 10.50 & 5.50 & 1.15 & 1.10 & & 1.10 & 2.60 & .90 & 6.30 & 3. \\
\hline & & & & & 1.10 & & 3.80 & 1.0 & & & & 4.85 \\
\hline
\end{tabular}

Daily gage height of Black Warrior River at Tuscaloosa, Ala., for 1890.

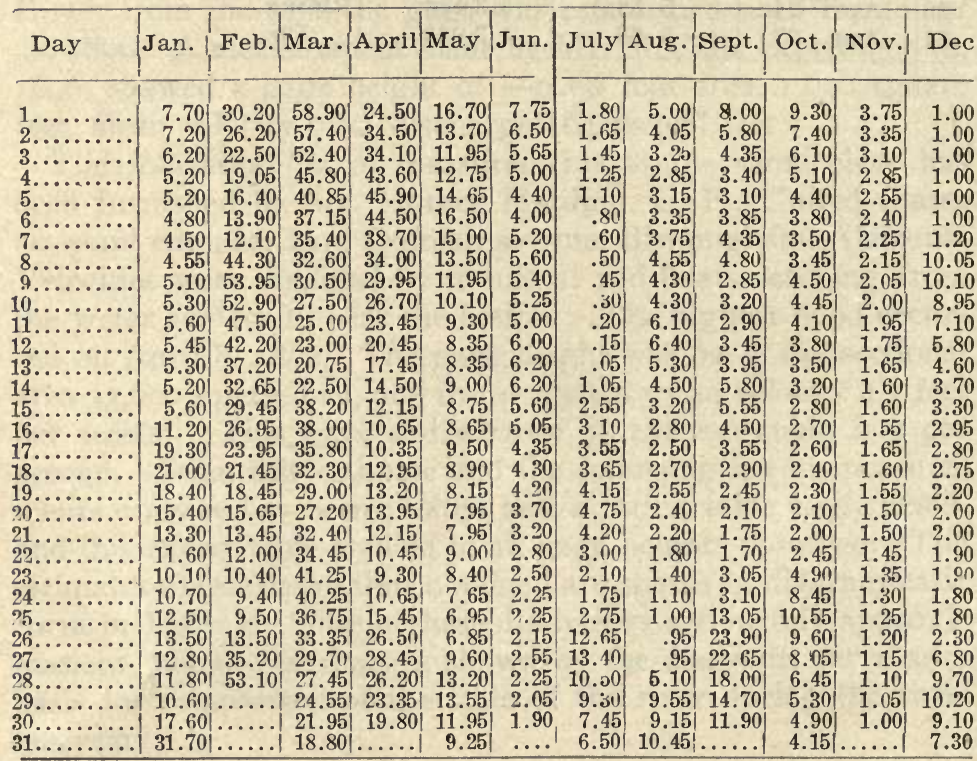


Daily gage heiglit of Black Warrior River at Tuscaloosa, Ala., for 1891.

\begin{tabular}{|c|c|c|c|c|c|c|c|c|c|c|c|c|}
\hline $\mathrm{D} z$ & Jan. & eb. & ar. & pril & May & Jun. & July! & Aug. & |Sept. & Oct. 1 & Nov. & Dec \\
\hline & & .20 & .00 & .00 & 5.70 & 1.80 & 2.10 & 6.50 & 1.00 & -0.40 & -0.80 & 3.40 \\
\hline & & & & & & 1.90 & .00 & 13.00 & 1.00 & -.30 & -.00 & 3.00 \\
\hline & & & & & & & .90 & & 1.00 & -.20 & -.80 & 2.40 \\
\hline & & & & & & & 1.80 & 16.00 & .80 & - & .80 & 6.20 \\
\hline & & & & & & & & 13.00 & .70 & -.20 & .80 & \\
\hline & & & & & & 1. & 1.30 & 9.50 & .60 & \& .20 & .80 & 21.50 \\
\hline & & & & & & & & 7.40 & .60 & -.40 & .80 & \\
\hline & & & & 50 & & 1. & 2. & 5.80 & .60 & .50 & .80 & 0.0 \\
\hline & & & & & & & & & .60 & -.60 & .80 & \\
\hline & & & & 20) & & 2. & 10.40 & & .60 & -.70 & +.70 & 17.00 \\
\hline & & & & & & 3. & & & .70 & -.80 & 2.10 & \\
\hline & & & & & & 10. & & & 1.00 & -.80 & 2.80 & 11.50 \\
\hline & & & & & & 10 & & & 1.20 & -.80 & 4.40 & 9.08 \\
\hline & & & & 0 & & & & & 1.20 & -.80 & 80 & 6.2 \\
\hline & & & & & & & & & 1.00 & -.60 & .50 & 7.0 \\
\hline & & & & & & & & & 1.00 & -.70 & .00 & 9.0 \\
\hline & & & & & & & & & .60 & -.70 & .80 & 11.0 \\
\hline & & & & & & & & & .20 & -.70 & & 11.2 \\
\hline & & & & & & & & & .10 & -.60 & 1.50 & 10.0 \\
\hline & & & & & & & & & .10 & $1-.70$ & & \\
\hline & & & & & & & 1.80 & & .10 & -.70 & 1.40 & 7. \\
\hline & & & & & & & & & .10 & -.60 & & 6 \\
\hline & & & & 10 & & & & & .10 & - & 00 & \\
\hline & & & & & & & 1.30 & 1.30 & .10 & -.50 & 12.30 & \\
\hline & & & & & & & & 1.20 & .00 & -.50 & & 7.5 \\
\hline & & & & 7. & & 4. & 2. & 1.10 & .00 & - & 10.80 & 13. \\
\hline & & & & & & & & 1.10 & -.20 & - & 7.20 & \\
\hline & & 26.50 & 18.20 & 8. & & & & 1.00 & $1-.20$ & - & 5.80 & \\
\hline & & & 19.40 & & & 2. & 2.00 & 1.00 & .40 & -.80 & 4.20 & 31. \\
\hline & & & 18.00 & 6.50 & 9.10 & 2.50 & & & &.- .80 & 3.80 & \\
\hline & 330 & & 17.00 & & 8.00 & & 2.40 & & & & & 22.1 \\
\hline
\end{tabular}

Daily gage heiglit of Black Warrior River at Tuscaloosa, Ala., for 189.2.

\begin{tabular}{|c|c|c|c|c|c|c|c|c|c|c|c|c|}
\hline Day & Jan. & Feb. & Mar. & April & May & Jun. & July & Aug. & Sept. & Oct. & Nov. & Dec \\
\hline & 17.90 & 21.20 & 10.70 & 21.80 & 12.00 & 3.40 & 4.60 & 4.80 & 9.30 & 3.90 & 0.40 & 5.90 \\
\hline & 15.90 & 10.50 & 10.30 & 18.30 & 11.20 & $3.2 \varnothing$ & 3.90 & 3.20 & 7.60 & 3.50 & .40 & 5.60 \\
\hline & 18.50 & 9.40 & 9.70 & 15.50 & 9.90 & 3.50 & 3.40 & 7.00 & 6.50 & 3.30 & 1.20 & 5.30 \\
\hline & 18.70 & 8.50 & 8.80 & 15.20 & 8.80 & 3.70 & 3.00 & 7.40 & 5.60 & 3.10 & 1.60 & 4.90 \\
\hline & 16.40 & 7.80 & 8.00 & 12.00 & 7.20 & 3.50 & 2.90 & 6.60 & 4.90 & 2.80 & 1.60 & 6.30 \\
\hline & 14.60 & 7.40 & 7.80 & 11.60 & 6.50 & 4.70 & 3.50 & 5.40 & 4.90 & 2.50 & 1.60 & 7.50 \\
\hline & 13.00 & 7.80 & 8.00 & 56.30 & 5.90 & 5.00 & 5.20 & 4.80 & 4.80 & 2.40 & 2.00 & 7.50 \\
\hline & 11.60 & 7.50 & 16.50 & 63.20 & 5.30 & 4.90 & 11.00 & 3.90 & 6.40 & 2.20 & 3.90 & 12.20 \\
\hline & 10.80 & 9.00 & 26.80 & 62.20 & 5.20 & 4.10 & 26.70 & 3.60 & 6.20 & 2.10 & 4.60 & 15.00 \\
\hline & 10.50 & 16.00 & 28.50 & 58.00 & 4.90 & 4.00 & 43.50 & 3.50 & 5.40 & 2.00 & 8.90 & 13.20 \\
\hline & 25.70 & 13.00 & 26.70 & 52.30 & 4.90 & 3.80 & 46.20 & 3.00 & 5.10 & 2.00 & 10.90 & 11.00 \\
\hline & 34.80 & 11.30 & 22.00 & 45.40 & 4.80 & 3.50 & 41.40 & 3.00 & 3.90 & 2.00 & 8.30 & 9.40 \\
\hline 13. & 53.00 & 10.80 & 18.00 & 40.70 & 4.70 & 3.30 & 38.30 & 3.30 & 3.40 & 2.00 & 7.60 & 8.00 \\
\hline & 57.40 & 9.50 & 15.80 & 36.50 & 4.60 & 2.80 & 37.50 & 3.10 & 3.30 & 1.90 & 5.80 & 7.40 \\
\hline 15. & 55.90 & 9.00 & 13.80 & 32.80 & 4.50 & 2.50 & 34.80 & 3.90 & 4.00 & 1.90 & 5.00 & 8.60 \\
\hline & 51.70 & 10.00 & 13.30 & 29.50 & 4.00 & 2.40 & 32.80 & $3.5(9)$ & 5.10 & 1.90 & 4.50 & 10.30 \\
\hline 17. & 45.00 & 11.80 & 11.20 & 27.001 & 3.70 & 2.00 & 41.40 & 3.50 & 4.90 & 1.80 & 4.00 & 13.10 \\
\hline & 40.10 & 11.00 & 12.90 & 24.50 & 3.60 & 1.80 & 38.00 & 6.00 & 4.30 & 1.70 & $4.2 v$ & 26.70 \\
\hline 19. & 36.60 & 9.90 & 24.00 & 22.40 & 4.30 & 2.10 & 33.00 & $9: 5 n$ & 6.00 & 1.00 & 4.40 & 28.40 \\
\hline & 41.50 & 10.50 & 25.50 & 20.20 & 4.60 & 3.90 & 29.00 & 11.50 & 18.30 & 1.00 & 4.70 & 28.40 \\
\hline & 41.00 & 13.50 & 22.90 & 18.10 & 6.50 & 7.40 & 28.30 & 9.50 & 23.90 & .90 & 5.00 & 36.50 \\
\hline & 36.801 & 18.00 & 20.00 & 15.90 & 6.30 & 10.80 & $2 y .30$ & 7.40 & 20.90 & .90 & 4.80 & 35.80 \\
\hline & 34.40 ! & 23.90 & 18.30 & $13.80 \mid$ & 6.00 & 10.70 & 25.10 & 10.20 & 17.00 & .90 & 4.60 & 31.50 \\
\hline & 31.00 & 21.20 & 22.00 & 12.30 & 6.00 & 8.40 & 21.50 & 12.30 & 13.30 & 1.00 & 4.20 & 27.00 \\
\hline & $28.50 \mid$ & 18.50 & 29.00 & 11.3 & 4.80 & 7.80 & 18.80 & 14.00 & 10.50 & 1.00 & & \\
\hline & 26.00 & 16.00 & 32.00 & 10.50 & 3.50 & 7.90 & $15.8 v$ & 14.20 & $8.00 \mid$ & .90 & 3.501 & 19.50 \\
\hline & 23.80 & 14.00 & 35.80 & 8.80 & 4.701 & 7.20 & 13.00 & 13.20 & & .80 & 3.301 & 16.00 \\
\hline & 21.50 & 12.50 & 34.00 & 7.90 & 4.00 & 6.10 & 10.50 & 11.50 & 5.00 & .70 & 3.20 & 13.60 \\
\hline & 19.00 & 11.50 & 30.50 & 7.70 & 3.80 & 5.20 & 7.80 & 12.00 & & .70 & 3.90 & 11.40 \\
\hline & 15.80 & & 26.80 & 10.50 & 3.50 & 5.40 & 6.20 & 11.50 & 4.30 & .50 & 5.90 & 9.8 \\
\hline & $|14.00|$ & & $|23.90|$ & 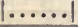 & & & 5.50 & 10.50 & & .00 & 1...... & 8.50 \\
\hline
\end{tabular}


Daily guge heigtit of Black Warrior River at Tuscaloosa, 1la, for 189.3.

\begin{tabular}{|c|c|c|c|c|c|c|c|c|c|c|c|c|}
\hline Day & Jan. & Feb. & Mar. & April & May & Jun. & July & Aug. & Sept. & Oct. & Nov. & Dec \\
\hline & 20 & 18.10 & 3.00 & .40 & 4.50 & 12.50 & 2.50 & 0.60 & 0.30 & 1.10 & 0.40 & 1. \\
\hline & & & & & & & & .70 & .20 & & .40 & \\
\hline & .80 & 14.00 & 19.70 & & .00 & & & 1.00 & .10 & 20 & .40 & \\
\hline & .70 & 12.80 & 20.50 & & & & & & .00 & & .40 & \\
\hline & .10 & 12.50 & 23.00 & & 2.20 & & & 1.30 & -.10 & & .40 & \\
\hline & ? & 11.80 & & & & & & 1.20 & -.10 & & .40 & \\
\hline & 8 & 11.2 & 2 & & 42.90 & 39. & & 1.30 & .00 & & .40 & \\
\hline & & & & & 40 & & & 1.20 & -.20 & 1.20 & :40 & \\
\hline & 7.00 & & 2 & & & & & 1.00 & $1.40^{\circ}$ & 1.00 & .40 & \\
\hline & & 10 & & & & & & .90 & 00 & .90 & .60 & \\
\hline & & & & & & & & .90 & & .80 & .60 & \\
\hline & 6.5 & 2 & & & & & & .80 & & .80 & & \\
\hline & & 2 & & & & & & .90 & 3.20 & .80 & .50 & 70 \\
\hline & 9 & 27. & 1 & & & & & 3.60 & & .70 & .60 & \\
\hline & 9 & 25. & 30 & 23. & & & 2.40 & 4.90 & & .70 & .60 & \\
\hline & .4 & 52 . & 1 & & & & & 5.10 & & .6 & .60 & \\
\hline & .20 & 55. & 13. & & 4.00 & & 1.70 & 4.70 & & .50 & .60 & \\
\hline & .80 & 54 . & 12. & & & & & & & .50 & .60 & \\
\hline & 0 & 51. & 11.30 & & 11.00 & & 30 & 2.80 & & .50 & .60 & \\
\hline & 1 & 46. & & & & & & & & .5 & .60 & \\
\hline & 1 & 41.8 & $9.90 \mid$ & & 6.90 & & .20 & 1.70 & & .50 & 0 & \\
\hline & .90 & & & & 6.20 & & & & & .50 & & \\
\hline & 1.20 & 34.50 & 8.8 & 10. & 60 & & & & & .40 & & \\
\hline & 12.90 & 31.30 & 12.30 & & & & & & & .4 & & \\
\hline & 15.9 & 28. & 22.20 & & & & & 1.00 & & .4 & 1.00 & \\
\hline & 19.2 & 25.90 & 22.50 & & 4.50 & & & .90 & & .4 & .90 & 1.70 \\
\hline & .00 & 23. & 20.0 & 10 & 4.3 & & 1.20 & .80 & & .4 & 1.0 & \\
\hline & 3.30 & 23.00 & & & 5.1 & & .9 & .80 & & .4 & 1.70 & \\
\hline & 2 & & 14.401 & 33. & 6.10 & 3. & .9 & .60 & 1. & .40 & 1.70 & \\
\hline & 22.1 & & & 29. & 12.90 & 0.80 & & & 1.10 & & 1.50 & \\
\hline & & & & & & & & & & & & \\
\hline
\end{tabular}

Daily gage heiglit of Black Warrior River at Tuscaloosa, Ala., for 1894.

\begin{tabular}{|c|c|c|c|c|c|c|c|c|c|c|c|c|}
\hline Day & Jan. & Feb. & Mar. & April & May & Jun. & July! & Aug. & Sept. & Oct. & Nov. & Dec \\
\hline & & 8.50 & 27.00 & 11.40 & 6.50 & & 2.50 & 1.20 & $\mathbf{5 . 8 0}$ & 0.55 & $\mid-0.05$ & -.05 \\
\hline & & 7.80 & 25.60 & 14.10 & 5.90 & $\begin{array}{l}1.60 \\
\end{array}$ & 1.80 & 1.30 & 4.60 & $\begin{array}{r}.45 \\
\end{array}$ & & -.05 \\
\hline & .90 & $\begin{array}{r}7.30 \\
790\end{array}$ & $\begin{array}{r}23.60 \\
71\end{array}$ & $\begin{array}{r}23.50 \\
24\end{array}$ & 5.90 & \begin{tabular}{|l|}
1.50 \\
1.35
\end{tabular} & 1.35 & $\begin{array}{l}1.40 \\
1.95\end{array}$ & 3.60 & .40 & -.20 & .00 \\
\hline & & 16.90 & 18.20 & 22.20 & 5.50 & $\begin{array}{l}1.33 \\
\end{array}$ & 8 & 2.30 & 2.45 & .25 & -.30 & \\
\hline & 6.40 & 22.60 & 15.90 & 20.60 & 5.20 & 1.20 & .60 & 2.40 & 2.05 & .20 & -.30 & .00 \\
\hline & 16.80 & 20.40 & 14.80 & 18.90 & 5.10 & \begin{tabular}{|l|}
1.20 \\
1
\end{tabular} & 1.00 & 2.00 & 1.85 & 15 & -.30 & .00 \\
\hline & 22.60 & $\begin{array}{r}17.80 \\
17.30\end{array}$ & $\mid \begin{array}{l}14.40 \\
13\end{array}$ & $\mid$\begin{tabular}{|c|}
16.30 \\
14.70
\end{tabular} & $\begin{array}{l}4.90 \\
4.50\end{array}$ & \begin{tabular}{|l|}
1.10 \\
95
\end{tabular} & 1.30 & 1.60 & & $\begin{array}{l}00 \\
10\end{array}$ & $|-.30|$ & +.05 \\
\hline & & 26.00 & .60 & & 4.10 & $\begin{array}{l}.90 \\
\end{array}$ & . & 1.1 & & .20 & & 50 \\
\hline & & 27.8 & .60 & & & 80 & .60 & .80 & & 25 & & 2.14 \\
\hline & 35.80 & 25.40 & 11.50 & 251 & 3.90 & 80 & .601 & 70 & & -.30 & $|-.30|$ & 5.70 \\
\hline & 30.90 & 29.90 & $|16.50|$ & $22.70 \mid$ & 4.30 & 70 & & & & .30 & $\mid-.30$ & $\begin{array}{ll}10 & 40\end{array}$ \\
\hline & 25.80 & $\begin{array}{l}32.10 \\
29.70\end{array}$ & $\mid \begin{array}{l}17.30 \\
15.80\end{array}$ & $\begin{array}{l}19.50 \\
16.30\end{array}$ & 6.0 & 60 & . .60 .60 & , & & -.30 & .19 & 11.20 \\
\hline & 3.60 . & 26.00 & 15.6 & & 5.7 & & .70 & & & & & $\begin{array}{l}8.40 \\
6.40\end{array}$ \\
\hline & & 22.3 & & & & 5 & & & & & & 4.90 \\
\hline & & 19.1 & & & & 5 & & & & 30 & & 3. \\
\hline & 1 & & & & & 60 & 7 & & & 30 & & 3. \\
\hline & & 1 & & & & 70 & .60 & & & 30 & & \\
\hline & .1 & 1 & & & & & 50 & & & 30 & & 2. \\
\hline & & & & & & 80 & 9 & & & 30 & & 2 \\
\hline & 8 & 00 & & & & 95 & 30 & & & 30 & & 2.0 \\
\hline & 9 & & & & & & 3 & & & 35 & & 1.8 \\
\hline & & & & & & & & & & 40 & & 1.7 \\
\hline & & & & & & 0 & & & & 40 & & 2.00 \\
\hline & & & & & & & & & & 45 & & 7.7 \\
\hline & & 3.80 & & & & & 3 & & 18 & & & 9.7 \\
\hline & & & & & & & 20 & & .9 & -.45 & & $8.40 \quad-$ \\
\hline & & & & & & & & & & & & 7.2 \\
\hline & 9.10 & & 12.70 & & 1.90 & & 1.20 & & & & $5-.05$ & 6.50 \\
\hline
\end{tabular}


Waily gage height of Black Warrior River at Tuscaloosa, Ala., for $189 j$.

\begin{tabular}{|c|c|c|c|c|c|c|c|c|c|c|c|c|}
\hline Day & Jan. & Feb. & Mar. & April| & May & Jun. & July & Aug. & Sept. & Oct. & Nov. & De \\
\hline & 5.10 & 24.10 & 8.60 & .20 & 15.20 & 7.00 & 4.80 & 1.80 & 1.90 & 0.10 & -0.04 & 0.75 \\
\hline & & & 13.20 & & & 5.90 & & 1.50 & 1.80 & .00 & +.07 & \\
\hline & 6.80 & 21.40 & 35.30 & 12.60 & 11.00 & 5.10 & 5.40 & 1.30 & 1.80 & .07 & .12 & 1.05 \\
\hline & 9.00 & 21.40 & 36.70 & 11.00 & 9.40 & 4.60 & 7.60 & 1.20 & 1.70 & .10 & .09 & 1.00 \\
\hline & 8.80 & 19.90 & 32.40 & 10.20 & ૪.30 & 4.20 & $17.4 n$ & 1.20 & 1.90 & .10 & .11 & .90 \\
\hline & 8.00 & 17.90 & 27.80 & ช. 40 & 1.40 & 6.70 & 17.70 & 1.20 & 2.60 & .10 & .25 & 1.30 \\
\hline & 7.40 & 16.50 & 23.80 & 7.90 & 7.00 & 5.70 & 18.40 & 1.10 & 3.05 & .10 & .31 & 1.50 \\
\hline & 35.00 & 16.80 & 20.60 & 20.50 & 8.20 & 5.10 & 15.70 & 1.00 & 3.70 & .00 & .30 & \\
\hline & 50.60 & 16.90 & 20.10 & 24.00 & 13.50 & 4.30 & 13.10 & .90 & 3.40 & .03 & .28 & 1. \\
\hline 10. & .30 & 15.80 & 19.30 & 21.20 & 19.70 & 3.80 & 11.10 & .97 & 2.90 & .07 & .70 & \\
\hline & .10 & 14.6 & 17.50 & 18.00 & 23.30 & 3.10 & 9.80 & 1.40 & 2.40 & .10 & 1.20 & \\
\hline 12. & .10 & 14.10 & 17.20 & 15.10 & 22.00 & 2.70 & 7.90 & 1.50 & 2.20 & . 14 & 1.10 & \\
\hline & .00 & 13.90 & 18.40 & 12.60 & 18.50 & 2.40 & 6.50 & 1.40 & 2.10 & .14 & 1.18 & 1.40 \\
\hline 14. & 29.80 & 12.80 & 24.90 & 11.00 & 15.20 & 2.20 & 5.30 & 1.30 & 2.00 & .06 & 1.35 & 1.3 \\
\hline 15. & .70 & 11.60 & 37.50 & 9.50 & 12.20 & 2.00 & 5.40 & 1.10 & 1.80 & .04 & 1.35 & 1. \\
\hline 16. & 23.40 & 10.50 & 47.40 & 8.60 & 9.70 & 2.00 & 5.60 & 1.30 & 1.60 & .08 & 1.28 & \\
\hline & 31.20 & 9.40 & 52.00 & 10.00 & 8.00 & 2.70 & 5.90 & 1.40 & 1.40 & .18 & 1.20 & 1. \\
\hline & 32.90 & 9.00 & 47.30 & 15.90 & 6.90 & 3.30 & 5.80 & 2.00 & 1.40 & .27 & 1.05 & 1. \\
\hline 19. & 29.20 & 9.00 & 42.10 & 15.80 & 6.30 & 4.20 & 5.10 & 4.00 & 1.30 & .31 & .88 & 1.0 \\
\hline & 25.80 & 9.50 & 38.80 & $14.0 v$ & 5.90 & 4.90 & 4.60 & 4.30 & 1.00 & .371 & .82 & 1. \\
\hline & 23.10 & 10.20 & 48.70 & 12.00 & 5.30 & 4.70 & 4.00 & 3.60 & 1.20 & .42 & .70 & \\
\hline & 1.60 & 10.90 & 51.30 & 10.40 & 4.80 & 4.30 & 3.60 & 2.80 & 1.50 & .45 & .60 & 2. \\
\hline & $21.60 \mid$ & 11.40 & 47.60 & 8.90 & 4.30 & 4.10 & 3.40 & 2.40 & 1.30 & -.50 & .50 & 3. \\
\hline & $19.80 \mid$ & 11.30 & 42.10 & 8.00 & 4.10 & 3.70 & 3.30 & 4.30 & 1.10 & -.35 & .75 & \\
\hline & 17.40 & 10.90 & 37.30 & 7.20 & 4.00 & 3.40 & 3.60 & 4.90 & .80 & .33 & .60 & \\
\hline & 16.70 & 10.30 & 32.80 & 7.00 & 4.30 & 3.00 & 3.70 & 4.00 & .60 & .53 & .38 & \\
\hline & 20.00 & 9.70 & 29.10 & 7.10 & 5.60 & 4.40 & 3.3 & 3.20 & .35 & .70 & .64 & \\
\hline & $21.20 \mid$ & 9.00 & 26.10 & $|12.50|$ & 11.00 & 5.4 & 2.9 & 2.40 & .10 & .68 & .65 & 21. \\
\hline & 22.00 & & 23.60 & 15.00 & 13.30 & 6.00 & 2. & 2.30 & .50 & .67 & .65 & \\
\hline & $27.4 \rho$ & & 21.10 & $16.40 \mid$ & 11.20 & 5.40 & 2.00 & 2.10 & .30 & -.50 & .60 & 13.1 \\
\hline $31 \ldots \ldots \ldots$ & $|27.10|$ & & $18.60 \mid$ & $|\ldots \ldots \ldots|$ & 8.90 & & 2.10 & 2.00 & & $|-.32|$ & & 11.67 \\
\hline
\end{tabular}

Daily gage height of Black Warrior River at Tuscaloosa, Ala., for 1896.

\begin{tabular}{|c|c|c|c|c|c|c|c|c|c|c|c|c|c|}
\hline Day & Jan. & Feb. & Mar. & April & May & Jun. & July! & Aug.| & & pt. 1 & Oct. 1 & ov. & Dec \\
\hline & 11.41 & 7.92 & $6.91 \mid$ & 10.61 & $18.24 \mid$ & 4.92 & 1.94 & .53 & & .38 & .52 & .78 & 7.70 \\
\hline & 10.26 & 8.51 & 7.66 & 14.78 & 33.30 & 4.28 & 1.71 & .80 & & .25 & .62 & .77 & 6.32 \\
\hline & 8.90 & 21.98 & 7.41 & 23.001 & 37.18 & 3.50 & 1.44 & 1.34 & & .15 & .66 & .42 & 4.63 \\
\hline & 7.60 & 33.12 & 6.74 & 23.50 & 30.88 & 5.44 & 1.48 & .89 & & .05 & .20 & .39 & 3.40 \\
\hline & 6.55 & 30.02 & 6.14 & 19.85 & $26.40 \mid$ & 5.24 & 1.30 & .54 & - & .06 & .78 & .33 & 2.60 \\
\hline & $5.74 !$ & 30.75 & 6.64 & $16.70 \mid$ & 21.93 & $4.3 n$ & 1.15 & .42 & & .15 & .74 & .19 & 2.05 \\
\hline & 5.09 & 35.92 & 10.18 & 14.26 & 16.88 & 3.62 & 1.32 & .50 & 1 - & .23 & .68 & .19 & 1.70 \\
\hline & 5.24 & 35.08 & 12.04 & $|12.55|$ & 13.95 & 3.27 & 2.20 & .60 & $1-$ & $.30 \mid$ & .40 & .17 & 1.47 \\
\hline & 5.76 & 36.21 & 14.89 & 11.20 & 10.80 & $6.6 \Gamma$ & 8.40 & .68 & - & .37 & .09 & .18 & 1.21 \\
\hline 10. & 6.461 & 36.52 & 13.69 & 10.02 & 8.60 & 8.18 & 6.98 & .64 & & .44 & .15 & .21 & 1.10 \\
\hline 11 & 6.581 & 33.65 & 12.45 & 9.04 & 7.111 & 18.39 & 5.37 & .55 & - & .44 & .28 & .30 & .89 \\
\hline 12. & 6.95 & 29.45 & 13.97 & 8.33 & 6.099 & 15.12 & 3.95 & .45 & & .46 & .28 & .20 & 84 \\
\hline 13. & 5.79 & 25.97 & 16.15 & 7.62 & 5.34 & 10.77 & 9.99 & .38 & - & .50 & .48 & .22 & 80 \\
\hline 14. & $5.26 !$ & 27.411 & | 15.35 & 7.86 & $4.77 !$ & 7.66 & 2.45 & .25 & $1-$ & .60 & .491 & 11 & 75 \\
\hline & $4.8 ? 1$ & $32.25 !$ & $13.56 \mid$ & 11.65 & 6.131 & 5.60 & 2.16 & .31 & i- & .59 & .46 & .10 & 1.20 \\
\hline 6. & 4.871 & 31.021 & 13.86 & $13.90 \mid$ & $5.45 !$ & 4.33 & $2.12 !$ & . 96 & & .59 & {$[-.43]$} & .01 & 1.25 \\
\hline & 6.031 & 97.301 & $22.3 n$ & 12.36 & 4.651 & 4.74 & 2.15 & .13 & $1-$ & .60 & 60 & .26 & 1.25 \\
\hline & 8.61! & $23.65 !$ & 27.75 & $10.57 \mid$ & $3.94 !$ & $4.2 n$ & 2.42 & .40 & & .60 & $-.63 \mid$ & .46 & \\
\hline & 9.141 & $9 \cap . \cap 9$ & 99.701 & 9.03 & 3.441 & 6.18 & 2.461 & .50 & $1-$ & .60 & .781 & .52 & 90 \\
\hline & 8.531 & 17.00 & 37.68 & 7.811 & $3.05 !$ & $6.0 n$ & 2. $94 !$ & .40 & & .611 & -.82 & .45 & \\
\hline & 7.871 & $14.45 \mid$ & 37.92 & 6.88 & 2.911 & 5.32 & 2.16 & .34 & $1-$ & .64 & .82 & .39 & 7 \\
\hline & 8.85 & $12.2 n !$ & 33.55 & 6.15 & 2.771 & & 1.90 & .29 & & .64 & $\mid-.84]$ & .381 & 1 \\
\hline 23 & $22.47 \mid$ & 10.391 & 29.12 & 5.56 & 2.871 & 4.46 & 1.71 & .22 & 1- & .36 & .84 & .38 & .20 \\
\hline & 29.261 & $9.13 \mid$ & 25.85 & 5.671 & 2.90 & 4.1 & 2.06 & 1.06 & $1-$ & .45 & $\mid-.78$ & .371 & .98 \\
\hline & $26.52 !$ & 8.601 & 23.54 & 5.76 & 2.871 & 4.04 & 2.53 & .95 & $1-$ & .61 & .80 & .371 & .92 \\
\hline & $29.44 \mid$ & 8.25 & 21.28 & 5.30 & 2.971 & & 2.171 & .79 & $1-$ & .64 & .82 & .37 & \\
\hline & $18.55 \mid$ & 7.9 م| & $|18.79|$ & 5.26 & 2.661 & 2.85 & $1.70 \mid$ & $.77 \mid$ & $1-$ & .711 & .82 & .98 & \\
\hline & 14.921 & 7.401 & 16.35 & 10.471 & 3.591 & 2.6 & 1.28 & 1.35 & $1-$ & .76 & .78 & . 991 & \\
\hline & 12.14 & 7.001 & $14.3 n$ & $16 . \cap \mathrm{C}$ & $6.5 \%$ & 9.54 & .94 & 1.30 & $1-$ & .67 & $-.80 \mid$ & .33 & .52 \\
\hline & 10.18 & ....... & 12.69 & 14.18 & 6.501 & 2.21 & .76 & .95 & & .55 & -.80 & 1.00 & .48 \\
\hline 31. & $8.88 !$ & & 11.40 & ....... & 5.781 & & .64 & .60 & & & $|-.78|$ & & .48 \\
\hline
\end{tabular}


The following discharge measurement was made by B. M. Hall in 1897 , at Tuscaloosa, Ala. :

January 12, gage height, 1.70 feet; discharge, 829 second-feet.

Daily gage heigint of Black Warrior River at Tuscaloosa, Ala., for $189 \%$.

\begin{tabular}{|c|c|c|c|c|c|c|c|c|c|c|c|c|}
\hline Day & an. & Teb. & Mar. & April & May & Jun. & July & ug. & Sept. & Oct. 1 & |Nov. & Des \\
\hline & 34 & 90 & (14) & .90 & 9.51 & 1.83 & -0.15 & 1.36 & 0.60 & -1.65 & $5 \mid-1.39$ & \\
\hline & & & & & & 1.75 & -.18 & 1.08 & .96 & -1.71 & $1-1.30$ & -1. \\
\hline & .4 & 11. & & 22.20 & 9. & 1.70 & .20 & .87 & 1.02 & -1.72 & -1.29 & \\
\hline & .9 & & & 1.11 & 7.66 & 2.01 & .18 & .62 & .92 & -1.75 & $5-1.31$ & +1 . \\
\hline & .24 & & 4 & 2.00 & 6.23 & 1.98 & .05 & .50 & .76 & $-\mathbf{1 . 7 9} \mid$ & $9 \mid-1.33$ & 13 \\
\hline & & & & 6.95 & 5.36 & & & .40 & .62 & -1 & -1.28 & 14 \\
\hline & & & & 5.32 & & & & .25 & .51 & -1.79 & $-1.27 \mid$ & 10 \\
\hline & & & & 0 & & & & .24 & .47 & & -1.27 & \\
\hline & 6 & & 5 & 0 & & & & 1.10 & .42 & -1 & -1.17 & \\
\hline & & & & & & & & 2.10 & .36 & & -1.13 & \\
\hline & & & & 2 & & & 53 & 3.26 & .29 & & -1.10 & \\
\hline & 1 & & & & 4 & & 42 & .22 & .23 & & $\mid-1.14$ & \\
\hline & .52 & 2 & & & 1 & 1.24 & 16 & 73 & .14 & & -1.17 & \\
\hline & .33 & 2 & & & & 1.11 & 86 & 2.27 & .11 & & -1.25 & \\
\hline & 1.23 & 20. & & & 6 & 95 & & & .10 & & -1.25 & \\
\hline & 8.2 & 16.8 & 30 & 18. & 9 & .85 & 1.16 & 1.28 & .06 & -1 & -1.27 & \\
\hline & .70 & & 1 & & 12. & .75 & .97 & 1.00 & .01 & & $\mid-1.33$ & 4. \\
\hline & 1 & 11 . & & & & .63 & 1.50 & .73 & .05 & & -1.35 & \\
\hline & & & & & & 1.30 & & & .36 & & & \\
\hline & & 8. & & & & $1.40^{\circ}$ & 50 & 1.53 & .75 & & -1 & \\
\hline & & & & & & 1.11 & & 1.78 & $81-.95$ & & & \\
\hline & .64 & 8.3 & & & & .80 & 30 & 1.58 & $8 \mid-1.07$ & -1.77 & $|-1.37|$ & 10 \\
\hline & .52 & 11. & & & & .55 & & 1.33 & & & & \\
\hline & & 20. & & & & & & 1.08 & & & & \\
\hline & 10.60 & 21. & & & & .23 & & 1.27 & & & & \\
\hline & 8.60 & 18. & 2 & & & .16 & & .97 & 7) -1.36 & -1 & -1.36 & 18.9 \\
\hline & & & & & & .12 & & .80 & & & & \\
\hline & 6.00 & 13.31 & 23 . & 5. & & .03 & 2.9 & .61 & $1-1.44$ & -1 & -1.28 & 13. \\
\hline & & & & & 2.28 & .00 & & .54 & & & $|-1.29|$ & 11.0 \\
\hline & 4.42 & & 17.52 & 5.90 & 2.09 & $1-.08$ & 1.80 & .46 & $6-1.55$ & -1.6 & $3 \mid-1.24 !$ & $4 ! \quad 0.38$ \\
\hline & $3.80 \mid$ & & 14.98 & & 2.00 & & 1.62 & .31 & & -1.63 & $\mid \ldots \ldots$ & 8.0 \\
\hline
\end{tabular}


Daily gage heiglit of Black Warrior River at Tuscaloosa, Ala., for 1898.

\begin{tabular}{|c|c|c|c|c|c|c|c|c|c|c|c|c|}
\hline Day & an. & Feb. & Mar. & pril & May & Jun. & July & Aug. & Sept. & Oct. & Nov. & Dec \\
\hline & .10 & & 3.67 & .50 & 1.34 & 0.23 & .66 & .45 & 1.20 & -0.60 & .90 & . \\
\hline & & & & & 9.82 & .08 & 43 & .95 & .82 & -.70 & & \\
\hline & & & 4. & & 8.67 & .23 & .30 & 2. & .70 & -.80 & .60 & \\
\hline & & 1 & 4. & & 7.68 & .27 & .53 & 2.80 & .30 & -.90 & .40 & \\
\hline & & 1 & 3.98 & & 6.88 & .27 & .16 & 2.96 & .00 & -1.00 & .00 & \\
\hline & 4. & 9.42 & 3.90 & 70 & 6.20 & .15 & .10 & 3.90 & .10 & -.90 & .30 & \\
\hline & 4. & & 3.5 & & 5.59 & 02 & -.18 & 3.40 & .30 & .90 & .50 & \\
\hline & & & 3.33 & & 5.00 & -.10 & -.04 & 3.10 & .20 & .70 & .40 & \\
\hline & 4.1 & & 3.12 & 0 & 4.50 & -.29 & -.04 & 3.98 & .00 & -.30 & .40 & \\
\hline & 3. & & 3.00 & 2 & 4.20 & -.41 & +.10 & 6.20 & .20 & +2.00 & .50 & \\
\hline & & & 2. & & 3.91 & -.54 & .88 & 12.20 & .40 & 3.30 & .50 & \\
\hline & & & 2.8 & & & -.62 & -.07 & 14.10 & .20 & .60 & .40 & \\
\hline & 6 & & 2.8 & 1 & 3.21 & -.71 & -.10 & 10 & .00 & .00 & & \\
\hline & & & & & & & -.07 & 7.30 & .30 & .40 & & \\
\hline & 11.9 & & 4.8 & 1 & 2.58 & -.53 & -.11 & 5.00 & .30 & 1.00 & 1.10 & \\
\hline & & & 8. & & 2.30 & -.38 & -.07 & 3.50 & .40 & .80 & & \\
\hline & & & 15. & & 2.08 & -.48 & -.14 & 2.62 & .60 & .4 & & \\
\hline & & & & & 1.97 & -.56 & .00 & 1.91 & .70 & .8 & & \\
\hline & & & & & 1.64 & -.30 & 1.24 & 1.50 & .70 & .8 & & \\
\hline & & 4.8 & & & 1.43 & $\{-.21$ & 1.30 & 1.46 & .80 & 1.20 & & \\
\hline & & & & & 1. & .00 & 1.12 & 1.12 & .90 & & & \\
\hline & & & & & 1. & .07 & .88 & 1.26 & .50 & & & \\
\hline & & & & & $1.0 n$ & .18 & & 1.20 & -.60 & & & \\
\hline & & & & & .93 & .30 & 1.08 & .90 & .70 & 80 & & \\
\hline & & & & & .78 & .17 & & .50 & .80 & 4.30 & 11. & \\
\hline & 4 & 3. & & 2 & .57 & .06 & 2. & 1.10 & -.90 & & & \\
\hline & & & & & .43 & 1.30 & 2.00 & 1.02 & -.90 & & & \\
\hline & & 3.72 & & & .43 & 3. 1.10 & 1.80 & .98 & -.80 & 2.3 & & \\
\hline & & & & & .52 & .46 & 2. & 1.30 & -.80 & 2.00 & & \\
\hline & 28.50 & & 13. & 13.03 & .44 & .68 & 2.55 & 1.52 & $2-.60$ & 1.40 & 5.30 & \\
\hline & 24.90 & & 25. & & .00 & & 2.86 & 1.56 & & 1.10 & & \\
\hline
\end{tabular}

The following measurements were made by B. M. Hall, and Prof. George S. Wilkins, of the Alabama University, in I899:

Measurements of Black Warrior River at Tuscaloosa, Ala.

\begin{tabular}{|c|c|c|c|c|c|}
\hline Date. & 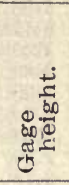 & 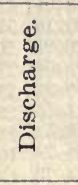 & Date. & 离 & 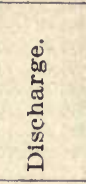 \\
\hline 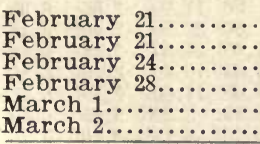 & $\begin{array}{l}19.36 \\
19.25 \\
22.85 \\
39.47 \\
35.50 \\
30.35\end{array}$ & $\begin{array}{l}12,855 \\
12,640 \\
16,216 \\
48,010 \\
24,988 \\
18,052\end{array}$ & 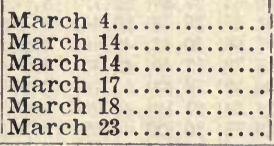 & $\begin{array}{l}23.70 \\
31.18 \\
34.37 \\
59.50 \\
56.40 \\
40.30\end{array}$ & $\begin{array}{r}12,609 \\
36,653 \\
40,331 \\
119,533 \\
86,410 \\
23,911\end{array}$ \\
\hline
\end{tabular}


Daily gage heigtht of Black Warrior Piver at Tuscaloosa, Ala., for 1899.

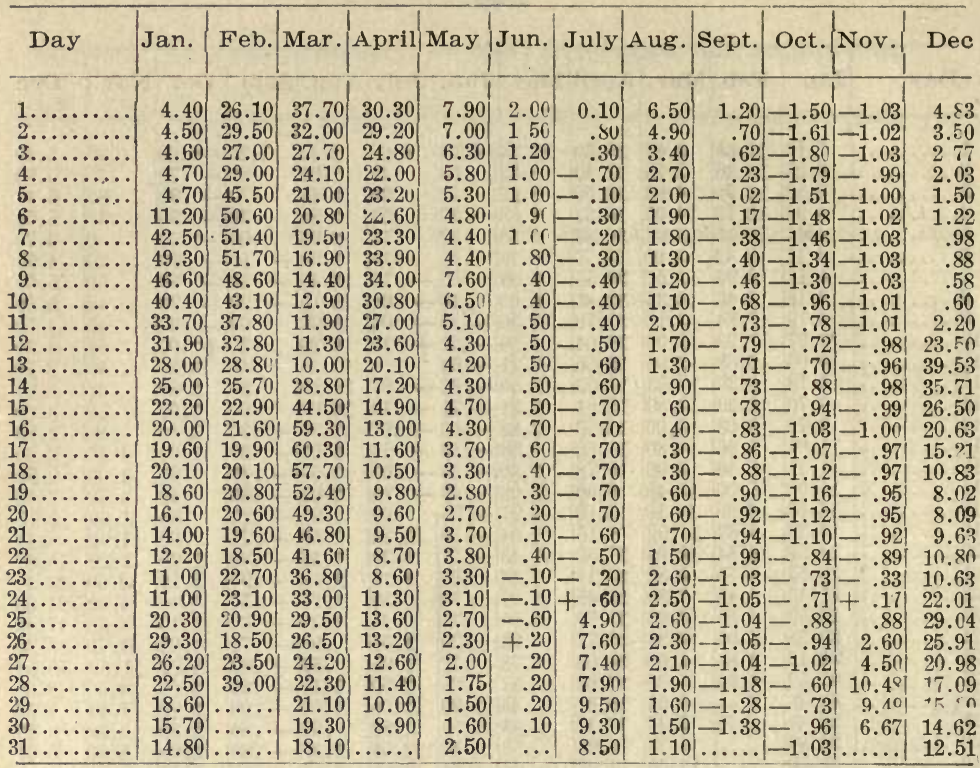

Daily gage height of Black Warrior River at Tuscaloosa, Ala., for 1900.

\begin{tabular}{|c|c|c|c|c|c|c|c|c|c|c|c|c|}
\hline Day & Jan. & Feb. & Mar. & Aprill & May & Jun. & July. & Aug. & Sept. & Oct. & Nov. & Dec \\
\hline & & & & & & 4.24 & 41.00 & 9.85 & 5.50 & 0.75 & $4.2^{n}$ & 1250 \\
\hline & $\begin{array}{l}9.48 \\
0.29\end{array}$ & 6.40 & $|25.44|$ & $\begin{array}{l}18.60 \\
150\end{array}$ & 18. & $\begin{array}{l}5 \mid 5.30 \\
513\end{array}$ & $\begin{array}{l}33.88 \\
9\end{array}$ & 8.00 & 5.00 & .80 & 5.10 & 9.65 \\
\hline & $\begin{array}{l}8.32 \\
7.10\end{array}$ & $\begin{array}{l}5.00 \\
5.71\end{array}$ & \begin{tabular}{|}
26.00 \\
23.30
\end{tabular} & $\begin{array}{l}15.80 \\
13.60\end{array}$ & \begin{tabular}{|l|}
16.33 \\
13.82
\end{tabular} & & 32.13 & $\begin{array}{l}6.501 \\
5.15\end{array}$ & $\begin{array}{l}4.07 \\
4.30\end{array}$ & . .65 & $\begin{array}{l}5.40 \\
5.55\end{array}$ & $\begin{array}{l}8.50 \\
790\end{array}$ \\
\hline & 6.18 & 6.40 & & 12.27 & 11.60 & 020.98 & 30.96 & 4.40 & 3.58 & 40 & 5.05 & 8.20 \\
\hline & 5.53 & 9.58 & & 12.17 & | $9.6 n$ & & 27.31 & 3.80 & $2.80 !$ & $40 \mid$ & 4.45 & 10.20 \\
\hline & 5.12 & 11.50 & & 11.44 & 8.2 & $0 \mid 20.92$ & 23.70 & 3.35 & 2.30 & .801 & 3.00 & 1065 \\
\hline & 4.84 & 10.53 & & 10.29 & 7.2 & $8 \mid 21.75$ & 20.80 & 3.00 & $1: 90$ & 1.80 & 3.50 & 9.65 \\
\hline & 4.60 & 12.23 & & 9.97 & 6.8 & & 18.45 & 2.501 & 1.50 & 3.90 & 3.10 & 8.5 \\
\hline & 4.50 & 20.60 & & & 6.5 & & 15.96 & 2.30 & 1.40 & 6.05 & 2.90 & 815 \\
\hline & 7.12 & 23.00 & & 26.35 & 6.1 & & 12.96 & 2.10 & 1.10 & 6.10 & 9.70 & 7.5 \\
\hline & 31.63 & 20.64 & 27.34 & 52.79 & 5. & 019.60 & 11.05 & 2.00 & .90 & 14.30 & 2.50 & 6.90 \\
\hline & 31.80 & $41.37 \mid$ & & 53.4 & 5.4 & & 10.60 & 1.75 & .80 & 22.50 & & \\
\hline & .18 & 47.96 & & 48. & 4.9 & & 12. & 1.50 & 1.40 & 21.85 & & 6. \\
\hline & 24.09 & 45.73 & 17.40 & 42.30 & $4.5^{p}$ & & $|10.35|$ & 1.40 & 5.45 & 16.40 & 2.20 & 6. \\
\hline & 20.12 & 40.23 & 16.63 & 37.10 & & & | $8.50 \mid$ & 1.50 & $|10.65|$ & $11.60 \mid$ & 2.10 & 6. \\
\hline & 16.50 & 34.74 & 21. & & 3. & & & 1.48 & 8.95 & 8.201 & 2.05 & 6. \\
\hline & .28 & 29.75 & & 64.05 & 3.5 & & 6.001 & 1.40 & 6.34 & 6.13 & & 5. \\
\hline & .80 & 25.881 & 18. & 62. & 3.52 & $2 \mid 24$ & 5.60 & 3.001 & 4.44 & 5.10 ! & 1.95 & 5. \\
\hline & 25.00 & 23.43 & 45. & & 3. & & 6.35 & $4.40^{\prime}$ & 3.35 & 4.30 & 3.90 & 5. \\
\hline & 32.60 & 19.90 & 51.0 & 56.10 & 3.65 & & $8.50 \mid$ & 3.75 & 2.60 & 3.60 & $\mid 8.40$ & 6. \\
\hline & 29.44 & 23.58 & $\mid 47.98$ & 51. & 3.50 & & 8.00 & 3.00 & 2.20 & $3.15 !$ & $|10.25|$ & 8. \\
\hline & 24.54 & 26.50 & 42.40 & & 3.35 & 5124. & $8.10 !$ & 2.50 & $1.87 \mid$ & 4.601 & | $12.5 n \mid$ & 1190 \\
\hline & 20.4 & $24.20 \mid$ & 38.24 & & $4.4 n$ & & $8.70 !$ & 2.55 & 1.65 & $7.30 \mid$ & 11.00 & 17.00 \\
\hline & 17.18 & & & & 7.5 & & $7.40 !$ & 2.10 & 1.60 & $\mid 10.40$ & $\begin{array}{l}9.4 \\
\end{array}$ & 18. \\
\hline & & 21.00 & 36. & & 7.68 & $8 \mid 56.35$ & 6.801 & 4.48 & 1.45 & 8.55 & 15. & \\
\hline & & & & 30.89 & 6.70 & & $9.2 n$ & 4.30 & $1.3 n$ & $6.75^{\prime}$ & 22.2 & 14.0 \\
\hline & 10.4 & 16.52 & 31.80 & 28.15 & 5.30 & 0 & 16.201 & 3.25 & 1.05 & 5.401 & $121 . \mathrm{nl}$ & \\
\hline & & & 28.85 & 25.73 & 4.25 & & 13.50 & $2.4 \mathrm{C}$ & .90 & & & \\
\hline & & & & & & & & & & & & \\
\hline & & & & & & & & & & & & \\
\hline
\end{tabular}


During 1901 the following discharge measurements were made on Black Warrior River at Tuscaloosa, Ala.:

1901.

Feb. 1-Hydrographer, K. T. Thomas; gage height, 15.10; discharge, 9,300 second-feet.

March 15-Hydrographer, K. T. Thomas; gage height, 18.72; discharge, 9,461 second-feet.

June 27-Hydrographer, K. T. Thomas; gage height, 1.77; discharge, 828 second-feet.

Daily gage heiglit of Black Warrior River at Tuscaloosa, Ala, for 1901.

\begin{tabular}{|c|c|c|c|c|c|c|c|c|c|c|c|c|}
\hline Day & Jan, & Feb. & Mar. & April & May & Jun. & July & Aug. & Sept. & Oct. & Nov. & Dec \\
\hline & 19.50 & 15.10 & 7.50 & 28.00 & 9.40 & 6.50 & 1.70 & 0.70 & 6.11 & 5.40 & $0 .\{5$ & 1.60 \\
\hline & 19.00 & 14.80 & 7.30 & 6.50 & & & 1.73 & 0.60 & 5.10 & 6.91 & 0.80 & 1.50 \\
\hline & 17.90 & 15.00 & 8.40 & 32.60 & 7.90 & 16.65 & 2. CO & 0.85 & 4.05 & 9.00 & 0.75 & 1.40 \\
\hline & 14.70 & 35.20 & 9.65 & 35.10 & 7.30 & 14.80 & $z_{.} r_{0}$ & 1.00 & 3.60 & 6.72 & 1.00 & 1.40 \\
\hline & 13.00 & 42.00 & 9.70 & 31.50 & 7.00 & 12.00 & 2.50 & 0.80 & 3.05 & 5.30 & 1.10 & 1.31 \\
\hline & 11.50 & 38.35 & 9.50 & 27.50 & 6.35 & 11.20 & 2.30 & 0.75 & 2.81 & 4.50 & 1.10 & 1.95 \\
\hline & 10.25 & 32.15 & 9.00 & 24.30 & 6.15 & 11.10 & 2.50 & 0.60 & 2.35 & 3.45 & 1.05 & 1.90 \\
\hline & 9.50 & 29.15 & 8.50 & 20.90 & 6.00 & 11.50 & 2.40 & 0.50 & 2.04 & 3.02 & 1.001 & 2.58 \\
\hline 9 & 8.75 & 28.05 & 8.05 & 17.90 & 5.70 & 9.40 & 2.25 & 0.35 & 1.90 & 2.50 & 0.95 & 2.60 \\
\hline 10 & 8.30 & 30.97 & 8.70 & 14.50 & 5.60 & $7.6 \mathrm{f}$ & 2.10 & 0.25 & 1.70 & 2.31 & 0.90 & 3.80 \\
\hline 11. & 17.40 & 29.75 & 29.50 & 12.50 & 5.00 & $6.5 r$ & 2.00 & 0.20 & 1.50 & 2.10 & 0.90 & 5.00 \\
\hline & 52.70 & 27.45 & 34.00 & 11.00 & 4.60 & 6.21 & 1.70 & 0.65 & 1.41 & 1.95 & 0.85 & 5.80 \\
\hline 13. & 56.50 & 25.15 & 28.50 & 10.00 & 5.00 & 6.40 & 1.30 & 0.65 & 1.30 & 3.41 & 1.00 & 5.70 \\
\hline & 53.25 & 22.50 & 23.50 & 11.35 & 6.60 & $55 f$ & 0.90 & 0.80 & 2.52 & 3.30 & 1.05 & 7.40 \\
\hline & 47.25 & 19.60 & 19.70 & 12.80 & 8.70 & 5.05 & 0.70 & 1.20 & 6.70 & 3.97 & 1.0 ก & 21. \\
\hline 16 & 41.45 & 17.45 & 16.00 & 13.00 & 8.30 & 4.80 & 0.60 & 5.70 & 8.00 & 3.8 ก & 0.98 & 40.75 \\
\hline & 36.30 & 15.70 & 13.00 & 11.70 & 6.80 & 4.50 & 0.43 & 17.00 & 6.10 & 3.511 & 0.9 ? & 35.00 \\
\hline & 31.85 & 13.70 & 11.05 & 12.80 & 5.50 & 4.10 & 2.60 & 26.30 & 13.21 & $3.0 \cap 1$ & ก.93 & $2^{7} .00$ \\
\hline & 28.15 & 12.50 & 10.00 & 25.70 & $4: 70$ & $3.8 \mathrm{f}$ & 4.00 & 22.70 & 16.00 & $2.7 n !$ & 1.10 & 2150 \\
\hline & 25.15 & 11.90 & 9.60 & 39.80 & 4.90 & 3.95 & $5.7 \mathrm{n}$ & 25.70 & 14.50 & 2.301 & 1.30 & 16.41 \\
\hline & 22.50 & 10.90 & 13. & 42.60 & 8.30 & 3.00 & 7.33 & 32.10 & 10.60 & 2.00 & 1.40 & 12.10 \\
\hline 2 & 19.85 & 9.95 & 16.50 & 38.00 & 17.30 & 2.75 & 6.00 & 32.50 & 7.50 & 1.811 & 1.70 & 9.95 \\
\hline & 17.55 & 9.20 & 15.70 & 32.80 & 19.80 & 2.30 & 4.50 & 31.97 & 5.70 ! & 1.61 & 2.0 & 7.00 \\
\hline & 15.35 & 8.75 & 14.00 & 28.41 & 17.50 & 2.20 & 3.20 & 26.80 & 4.51 & 1.45 & 2.05 & 6.80 \\
\hline & 14.60 & 8.65 & 13.80 & 24.40 & 14.10 & 2.00 & 2.50 & 22.40 & 3.90 & 1.31 & 2.00 & 7.30 \\
\hline & 18.00 & 8.50 & 28.50 & 21.00 & 11.30 & 1.97 & 2.00 & 18.30 & 3.40 & 1.20 & 1.95 & 8.24 \\
\hline & 17.20 & $8.2 n$ & 37.25 & 17.90 & 9.30 & 1.85 & 1.50 & 14.10 & 2.91 & 1.05 & 1.951 & 9.80 \\
\hline & 15.50 & 7.90 & 34.50 & 14.90 & 8.15 & 1.70 & 1.05 & $\begin{array}{l}9.85 \\
\end{array}$ & 2.60 & 0.81 & $9 . n^{\prime \prime} 1$ & 0.96 \\
\hline 2 & 14.80 & & 29.00 & 12.50 & 7.00 & 1.65 & 0.90 & $7.9^{n !}$ & 2.911 & $n .55$ & 1.951 & - 630 \\
\hline 30 & 15.10 & & 24.30 & 10.90 & 6.90 & 1.65 & 0.80 & 7.41 & 4.801 & 0.90 & 1.911 & $4^{\circ} 00$ \\
\hline 31 & $14.6 n \mid$ & & 24.85 & |....... & 6.35 & 1...... & $0.6^{n !}$ & 7.10 ! & .....। & 0.90 & $1 \times . . .1 .1$ & 49.00 \\
\hline
\end{tabular}


Rating table for Black Warrior River at Tuscaloosa, Ala.

[This table is applicable from Jan. 1, 1895, to Dec. 31, 1901.]

\begin{tabular}{|c|c|c|c|c|c|c|c|}
\hline 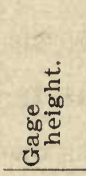 & 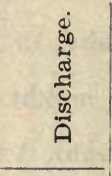 & ס & 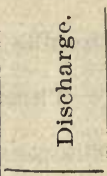 & 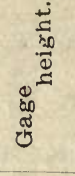 & 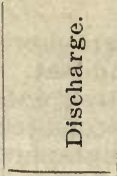 & & 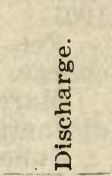 \\
\hline Feet. & Second $f t$. & H'ee'. & Second ft & Feet. & Secımd ft. & Feet. & Second ft. \\
\hline-1.0 & 90 & 6.0 & 3,110 & 13.0 & 6,995 & 20.0 . & 10,880 \\
\hline-0.9 & 105 & 6.1 & 3,160 & 13.1 & 7,045 & 20.1 & 10,950 \\
\hline-0.8 & 120 & 6.2 & 3,210 & 13.2 & 7,095 & 20.2 & 11,020 \\
\hline-0.7 & 140 & 6.3 & 3,260 & 13.3 & 7,145 & 20.3 & 11,090 \\
\hline-0.6 & 160 & 6.4 & 3,310 & 13.4 & 7,195 & 20.4 & 11,160 \\
\hline-0.5 & 180 & 6.5 & 3,370 & 13.5 & 7,255 & 20.5 & 11,230 \\
\hline-0.4 & 200 & 6.6 & 3,430 & 13.6 & 7,315 & 20.6 & 11,305 \\
\hline-0.3 & 220 & 6.7 & 3,490 & 13.7 & 7,375 & 20.7 & 11,380 \\
\hline-0.2 & 240 & 6.8 & 3,550 & 13.8 & 7,435 & 20.8 & 11,455 \\
\hline-0.1 & 260 & 6.9 & 3,610 & 13.9 & 7,495 & 20.9 & 11,530 \\
\hline 0.0 & 280 & 7.0 & 3,665 & 14.0 & 7,550 & 21.0 & 11,600 \\
\hline 0.1 & 310 & 7.1 & 3,715 & 14.1 & 7,600 & 21.1 & 11,690 \\
\hline 0.2 & 340 & 7.2 & 3,765 & 14.2 & 7,650 & 21.2 & 11,780 \\
\hline 0.3 & 370 & 7.3 & 3,815 & 14.3 & 7,700 & 21.3 & 11,870 \\
\hline 0.4 & 400 & 7.4 & 3,865 & 14.4 & 7,750 & 21.4 & 11,960 \\
\hline 0.5 & 430 & 7.5 & 3,925 & 14.5 & 7,810 & 21.5 & 12,050 \\
\hline 0.6 & 460 & 7.6 & 3,985 & 14.6 & 7,870 & 21.6 & 12,140 \\
\hline 0.7 & 490 & 7.7 & 4,045 & 14.7 & 7,930 & 21.7 & 12,230 \\
\hline 0.8 & 530 & 7.8 & 4,105 & 14.8 & 7,990 & 21.8 & 12,320 \\
\hline 0.9 & 565 & 7.9 & 4,165 & 14.9 & 8,050 & 21.9 & 12,410 \\
\hline 1.0 & 600 & 8.0 & 4,220 & 15.9 & 8,105 & 22.0 & 12,500 \\
\hline 1.1 & 635 & 8.1 & 4,270 & 15.1 & 8,155 & 22.1 & 12,600 \\
\hline 1.2 & 670 & 8.2 & 4,320 & 15.2 & 8,205 & 22.2 & 12,700 \\
\hline 1.3 & 710 & 8.3 & 4,370 & 15.3 & 8,255 & 22.3 & 12,800 \\
\hline 1.4 & 750 & 8.4 & 4,420 & 15.4 & 8,305 & 22.4 & 12,900 \\
\hline 1.5 & 790 & 8.5 & 4,480 & 15.5 & 8,365 & 22.5 & 13,000 \\
\hline 1.6 & 830 & 8.6 & 4,540 & 15.6 & 8,425 & 22.6 & 13,100 \\
\hline 1.7 & 870 & 8.7 & 4,600 & 15.7 & 8,485 & 22.7 & 13,200 \\
\hline 1.8 & 910 & 8.8 & 4,660 & 15.8 & 8,545 & 22.8 & 13,300 \\
\hline 1.9 & 955 & 8.9 & 4,720 & 15.9 & 8,605 & $22: 9$ & 13,400 \\
\hline 2.0 & 1,000 & 9.0 & 4,775 & 16.0 & 8,660 & 23.0 & 13,500 \\
\hline 2.1 & 1,045 & 9.1 & 4,825 & 16.1 & 8,710 & 23.1 & 13,620 \\
\hline 2.2 & 1,090 & 9.2 & 4,975 & 16.2 & 8,760 & 23.2 & 13,740 \\
\hline 2.3 & 1,135 & 9.3 & 4,925 & 16.3 & 8,810 & 23.3 & 13,860 \\
\hline 2.4 & 1,180 & 9.4 & 4,975 & 16.4 & 8,860 & 23.4 & 13,980 \\
\hline 2.5 & 1,225 & 9.5 & 5,035 & 16.5 & 8,920 & 23.5 & 14,100 \\
\hline 2.6 & 1,270 & 9.6 & 5,095 & 16.6 & 8.980 & 23.6 & 14,220 \\
\hline 2.7 & 1,320 & 9.7 & 5,155 & 16.7 & 9,040 & 23.7 & 14,340 \\
\hline 2.8 & 1,370 & 9.8 & 5,215 & 16.8 & 9,100 & 23.8 & 14,460 \\
\hline 2.9 & 1,420 & 9.9 & 5,275 & 16.9 & 9,160 & 23.9 & 14,580 \\
\hline 3.0 & 1,470 & 10.0 & 5,330 & 17.0 & 9,215 & 24.0 & 14,700 \\
\hline 3.1 & 1,520 & 10.1 & 5,380 & 17.1 & 9,265 & 24.1 & 14,830 \\
\hline 3.2 & 1,570 & 10.2 & 5,430 & 17.2 & 9,315 & 24.2 & 14,960 \\
\hline 3.3 & 1,620 & 10.3 & 5,480 & 17.3 & 9,365 & 24.3 & 15,090 \\
\hline 3.4 & 1,670 & 10.4 & 5,530 & 17.4 & 9,415 & 24.4 & 15,200 \\
\hline
\end{tabular}




\begin{tabular}{|c|c|c|c|c|c|c|c|}
\hline 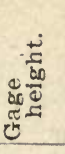 & 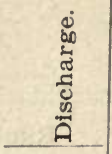 & 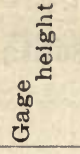 & 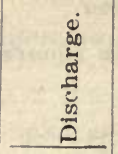 & 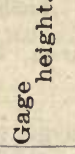 & 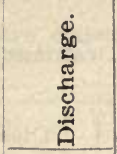 & 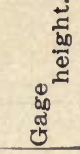 & 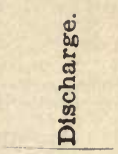 \\
\hline Feet. & Second $f t$. & Feet. & Second $f t$. & Feet. & sicond $f t$. & Feet. & Second $f t$. \\
\hline 3.5 & 1,725 & 10.5 & 5,590 & 17.5 & 9,475 & 24.5 & 15,350 \\
\hline 3.6 & 1,780 & 10.6 & 5,650 & 17.6 & 9,535 & 24.6 & 15,480 \\
\hline 3.7 & 1,835 & 10.7 & 5.710 & 17.7 & 9,595 & 24.7 & 15,610 \\
\hline 3.8 & 1,890 & 10.8 & 5,770 & 17.8 & 9,655 & 24.8 & 15,740 \\
\hline 3.9 & 1,945 & 10.9 & 5,830 & 17.9 & 9,715 & 24.9 & 15,870 \\
\hline 4.0 & 2,000 & 11.0 & 5,885 & 18.0 & 9,770 & 25.0 & 16,000 \\
\hline 4.1 & 2,055 & 11.1 & 5,935 & 18.1 & 9,820 & 26.0 & 17,600 \\
\hline 4.2 & 2,111 & 11.2 & 5,985 & 18.2 & 9,870 & 28.0 & 21,500 \\
\hline 4.3 & 2,166 & 11.3 & 6,035 & 18.3 & 9,920 & 30.0 & 26,500 \\
\hline 4.4 & 2,222 & 11.4 & 6,085 & 18.4 & 9,970 & 32.0 & 31,700 \\
\hline 4.5 & 2,277 & 11.5 & 6,145 & 18.5 & 10,030 & 34.0 & 38,000 \\
\hline 4.6 & 2,333 & 11.6 & 6,205 & 18.6 & 10,090 & 36.0 & 45,000 \\
\hline 4.7 & 2,388 & 11.7 & 6,265 & 18.7 & 10,150 & 38.0 & 53,000 \\
\hline 4.8 & 2,444 & 11.8 & 6,325 & 18.8 & 10,210 & 40.0 & 61,000 \\
\hline 4.9 & 2,500 & 11.9 & 6,385 & 18.9 & 10,270 & 42.0 & 69,000 \\
\hline 5.0 & 2,555 & 12.0 & 6,440 & 19.0 & 10,325 & 44.0 & 77000 \\
\hline 5.1 & 2,610 & 12.1 & 6,490 & 19.1 & 10,375 & 46.0 & 85,000 \\
\hline 5.2 & 2,666 & 12.2 & 6,540 & 19.2 & 10,425 & 48.0 & 93,000 \\
\hline 5.3 & 2,721 & 12.3 & 6,590 & 19.3 & 10,475 & 50.0 & 101,000 \\
\hline 5.4 & 2,777 & 12.4 & 6,640 & 19.4 & 10,525 & 52.0 & 109,000 \\
\hline 5.5 & 2,832 & 12.5 & 6,700 & 19.5 & 10,585 & 54.0 & $117,00 \mathrm{C}$ \\
\hline 5.6 & 2,888 & 12.6 & 6,760 & 19.6 & 10,645 & 55.0 & 121,000 \\
\hline 5.7 & 2,943 & 12.7 & 6,820 & 19.7 & 10,705 & & \\
\hline 5.8 & 3,000 & 12.8 & 6,880 & 19.8 & 10,765 & & \\
\hline 5.9 & 3,054 & 12.9 & 6,940 & 19.9 & 10,825 & & \\
\hline
\end{tabular}

NoTE.-This table applied to the foregoing "Daily gage heights" gives the cubic feet per second flowing in the river on each date for which the gage height is given. 
Estimated monthly discharge of Black Warrior River at Tuscaloosa, Alabama.

[Drainage area, 4,900 square miles.]

\begin{tabular}{|c|c|c|c|c|c|c|}
\hline \multirow[b]{2}{*}{ Month. } & \multicolumn{3}{|c|}{$\begin{array}{l}\text { Discharge in second- } \\
\text { feet. }\end{array}$} & \multirow[b]{2}{*}{$\begin{array}{l}\text { Total in } \\
\text { acre- } \\
\text { feet. }\end{array}$} & \multicolumn{2}{|c|}{ Run-off. } \\
\hline & 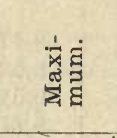 & 运完 & $\stackrel{\Xi}{\Xi}$ & & 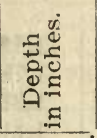 & 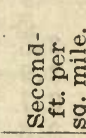 \\
\hline $189 \overline{.}$ & & & & & & \\
\hline January . & 103,400 & 2,777 & 25,464 & $1,565,720$ & 6.00 & 5.20 \\
\hline February $\ldots \ldots \ldots$ & 14,830 & 4,775 & 7,603 & 422,249 & 1.61 & 1.55 \\
\hline March $\ldots \ldots \ldots \ldots$ & 109,000 & 4,540 & 39,977 & $2,458,106$ & 9.42 & 8.16 \\
\hline April $\ldots \ldots \ldots \ldots$. & 14,700 & 3,665 & 6,895 & 410,280 & 1.57 & 1.41 \\
\hline May $\ldots \ldots \ldots \ldots$ & 13,860 & 2,000 & 5,511 & 338,860 & 1.29 & 1.12 \\
\hline June ........... & 3,665 & 1,000 & 2,133 & 126,922 & 0.49 & 0.44 \\
\hline July $\ldots \ldots \ldots \ldots \ldots$ & 9,970 & 1,000 & 3,581 & 220,189 & 0.84 & 0.73 \\
\hline August $\ldots \ldots \ldots \ldots$ & 2,500 & 565 & 1,098 & 67,514 & 0.25 & 0.22 \\
\hline September ....... & 1,835 & 310 & 883 & 52,542 & 0.20 & 0.18 \\
\hline October $\ldots \ldots \ldots$ & 310 & 140 & 233 & 14,327 & 0.06 & 0.05 \\
\hline November ........ & 750 & 280 & 488 & 29,038 & 0.11 & 0.10 \\
\hline December ........ & 11,600 & 530 & 2,021 & 124,267 & 0.47 & 0.41 \\
\hline 1896. & & & & & & \\
\hline January ......... & 24,610 & 2,444 & 5,981 & 367,757 & 1.41 & 1.22 \\
\hline February $\ldots \ldots \ldots$. & 47,000 & 3,665 & 19,161 & $1,102,153$ & 4.22 & 3.91 \\
\hline March...$\ldots \ldots \ldots$ & 52,600 & 3,160 & 12,996 & 799,093 & 3.06 & 2.65 \\
\hline April $\ldots \ldots \ldots \ldots$ & 14,100 & 2,721 & 6,072 & 361,309 & 1.38 & 1.24 \\
\hline May $\ldots \ldots \ldots \ldots \ldots$ & 49,800 & 1,370 & 7,420 & 456,238 & 1.74 & 1.51 \\
\hline June...$\ldots \ldots \ldots$ & 9,970 & 1,090 & 2,910 & 173,157 & 0.65 & 0.59 \\
\hline July $\ldots \ldots \ldots \ldots$ & 4,420 & 460 & 1,232 & 75,753 & 0.29 & 0.25 \\
\hline August $\ldots \ldots \ldots \ldots$ & 750 & 310 & 478 & 29,391 & 0.12 & 0.10 \\
\hline September ........ & 400 & 120 & 201 & 11,960 & 0.04 & 0.04 \\
\hline October ........... & 260 & 120 & 157 & 9,654 & 0.03 & 0.03 \\
\hline Nóvember $\ldots \ldots \ldots$ & 600 & 120 & 307 & 18,268 & 0.07 & 0.06 \\
\hline December ......... & 4,045 & 430 & 955 & 58,721 & 0.22 & 0.19 \\
\hline 1897. & & & & & & \\
\hline January . & 10,500 & 385 & 3,493 & 214,775 & 0.82 & 0.71 \\
\hline February ........ & 17,440 & 1,945 & 8,409 & 467,010 & 1.79 & 1.72 \\
\hline March $\quad \therefore \ldots \ldots \ldots$ & 120,080 & 4,540 & 52,883 & $|3,251,650|$ & 12.44 & 10.79 \\
\hline April $\ldots \ldots \ldots \ldots$ & 25,285 & 2,610 & 9,657 & $|574,630|$ & 2.20 & 1.97 \\
\hline May $\ldots \ldots \ldots \ldots$ & 11,195 & 1,000 & 3,600 & 221,355 & 0.84 & 0.73 \\
\hline June $\ldots \ldots \ldots \ldots$ & 1,697 & 260 & 715 & 42,545 & 0.17 & 0.15 \\
\hline July $\ldots \ldots \ldots \ldots \ldots$ & 7,810 & 240 & 1,809 & 111,230 & 0.43 & 0.37 \\
\hline August $\ldots \ldots \ldots \ldots$ & 1,595 & 355 & 701 & 43,100 & 0.16 & 0.14 \\
\hline September ........ & 600 & 102 & 295 & 17,555 & 0.07 & 0.06 \\
\hline October ........... & 102 & 90 & 93 & 5,718 & 0.02 & 0.02 \\
\hline November ....... & 125 & 107 & 115 & 6,843 & 0.03 & 0.02 \\
\hline $\begin{array}{c}\text { December } . . . \ldots \ldots \\
1898 .\end{array}$ & 29,000 & 115 & 5,549 & 341,195 & 1.30 & 1.13 \\
\hline January ......... & 75,000 & 1,972 & 16,577 & $\mid 1,019,287$ & 3.90 & 3.38 \\
\hline February ......... & 12,230 & 1,752 & 3,902 & 216,706 & 0.83 & 0.80 \\
\hline
\end{tabular}


Estimated monthly discharge of Black Warrior River at Tuscaloosa, Alabama.

[Drainage area, 4,900 square miles.]

\begin{tabular}{|c|c|c|c|c|c|c|}
\hline \multirow[b]{2}{*}{ Month. } & \multicolumn{3}{|c|}{$\begin{array}{l}\text { Discharge in second- } \\
\text { feet. }\end{array}$} & \multirow[b]{2}{*}{$\begin{array}{l}\text { Total in } \\
\text { acre-ft. }\end{array}$} & \multicolumn{2}{|c|}{ Run-off. } \\
\hline & 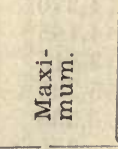 & 咅 & 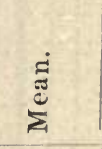 & & 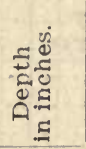 & 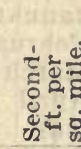 \\
\hline March a........... & 17,120 & 1,370 & 3,626 & 222,955 & 0.85 & 0.74 \\
\hline April ............ & 55,800 & 4,295 & 15,620 & 929,452 & 3.56 & 3.19 \\
\hline May.... & 6,060 & 370 & 1,766 & 108,589 & 0.41 & 0.36 \\
\hline June $\ldots$ & 710 & 160 & 303 & 18,030 & 0.07 & 0.06 \\
\hline July $\ldots \ldots \ldots \ldots$ & 1,395 & 250 & 549 & 33,757 & 0.13 & 0.11 \\
\hline August ...... & 7,600 & 430 & 1,785 & 109,756 & 0.41 & 0.36 \\
\hline September ....... & 670 & 140 & 252 & 14,995 & 0.06 & 0.05 \\
\hline October .......... & 2,444 & 130 & 880 & 54,109 & 0.21 & 0.18 \\
\hline November ....... & 6,265 & 280 & 1,626 & 96,754 & 0.37 & 0.33 \\
\hline December ........ & 14,580 & 1,045 & 3,763 & 231,379 & 0.89 & 0.77 \\
\hline 1899. & & & & & & \\
\hline January & $\begin{array}{l}81,375 \\
90,375\end{array}$ & $\begin{array}{r}2,222 \\
10,030\end{array}$ & $\begin{array}{l}18,118 \\
30\end{array}$ & $1,114,033$ & $\begin{array}{l}4.27 \\
6.57\end{array}$ & $\begin{array}{l}3.70 \\
6.31\end{array}$ \\
\hline & $\begin{array}{r}90,375 \\
122,625\end{array}$ & $\begin{array}{r}10,030 \\
5,330\end{array}$ & $\begin{array}{l}30,923 \\
35,308\end{array}$ & $\begin{array}{l}1,717,376 \\
2,171,004\end{array}$ & $\begin{array}{l}6.57 \\
8.31\end{array}$ & $\begin{array}{l}6.31 \\
7.21\end{array}$ \\
\hline $\begin{array}{l}\text { March } \quad \ldots \ldots \ldots \ldots \\
\text { April } \quad \ldots \ldots \ldots \ldots\end{array}$ & $\begin{array}{r}122,020 \\
32,800\end{array}$ & 4,540 & $\begin{array}{l}35,308 \\
11,901\end{array}$ & $\begin{array}{r}2,17,004 \\
708,158\end{array}$ & 2.71 & 2.43 \\
\hline & 4,165 & 790 & 2,092 & 128,632 & 0.49 & 0.43 \\
\hline June ........... & 1,000 & 175 & 448 & 26,658 & 0.10 & 0.09 \\
\hline July $\ldots \ldots \ldots \ldots$ & 5,035 & 160 & 1,111 & 68,313 & 0.26 & 0.23 \\
\hline August $\ldots \ldots \ldots \ldots$ & 3,370 & 370 & 963 & 59,213 & 0.23 & 0.20 \\
\hline September ..... & 670 & 110 & 200 & 11,901 & 0.04 & 0.04 \\
\hline October $\ldots \ldots \ldots$. & 175 & 92 & 130 & 7,993 & 0.03 & 0.03 \\
\hline November ........ & 5,590 & 127 & 721 & 42,902 & 0.17 & 0.15 \\
\hline December ........ & 47,650 & 460 & 8,880 & 546,010 & 2.09 & 1.81 \\
\hline 1900. & & & & & & \\
\hline January . & 29,760 & 2,277 & 9,857 & 606,083 & 2.01 & 2.32 \\
\hline February & 76,312 & 2,555 & 18,356 & $\mid 1,019,440$ & 3.75 & 3.90 \\
\hline March .. & 87,750 & 8,280 & 27,105 & $1,666,623$ & 5.53 & 6.37 \\
\hline April $\ldots$ & 136,687 & 5,302 & 48,426 & $2,881,547$ & 9.88 & 11.02 \\
\hline May $\ldots \ldots \ldots \ldots$. & 11,825 & 1,645 & 3,702 & 227,627 & 0.76 & 0.88 \\
\hline June $\ldots . . . \ldots \ldots$ & 115,312 & 2,138 & 32,614 & $1,940,668$ & 6.66 & 7.43 \\
\hline July & 52,000 & 2,888 & 10,952 & 673,412 & 2.24 & 2.59 \\
\hline August $\ldots \ldots \ldots \ldots$ & 5,245 & 750 & 1,674 & 102,930 & 0.34 & 0.39 \\
\hline September ........ & 5,680 & 512 & 1,580 & 94,017 & 0.32 & 0.36 \\
\hline October $\ldots \ldots \ldots$. & 13,000 & 355 & 3,382 & 207,951 & 0.69 & 0.80 \\
\hline November ....... & 12,700 & $977 !$ & 3,701 & 220,225 & 0.76 & 0.85 \\
\hline December ........ & 10,150 & 2,721 & 5,119 & 314,755 & 1.05 & 1.21 \\
\hline The year ....... & 136,687 & 355 & 13,872 & $\mid 9,955,278$ & 2.83 & 38.12 \\
\hline
\end{tabular}


Estimated monthly discharge of Black Warrior River at Tuscaloosa, Alabama.

[Drainage area, 4,900 square miles.]

\begin{tabular}{|c|c|c|c|c|c|}
\hline \multirow[b]{2}{*}{ Month. } & \multicolumn{3}{|c|}{$\begin{array}{l}\text { Discharge in } \\
\text { second-fert. }\end{array}$} & \multicolumn{2}{|c|}{ Run-off. } \\
\hline & $\begin{array}{l}\text { Maxi- } \\
\text { mum. }\end{array}$ & $\begin{array}{l}\text { Mini- } \\
\text { mum. }\end{array}$ & Mean. & $\begin{array}{c}\text { seeond- } \\
\text { feet per } \\
\text { square } \\
\text { mile. }\end{array}$ & $\begin{array}{l}\text { Depth } \\
\text { in } \\
\text { inches }\end{array}$ \\
\hline 1901. & & & & & \\
\hline January ... & $\mathbf{1 0 8 , 3 7 5}$ & 4,370 & 22,938 & 4.68 & 5.39 \\
\hline February $\ldots \ldots \ldots \ldots \ldots \ldots$ & 55,100 & 4,165 & 15,094 & 3.08 & 3.21 \\
\hline $\operatorname{March} \ldots \ldots \ldots \ldots \ldots \ldots \ldots$ & 40,900 & 3,815 & 11,947 & 2.44 & 2.81 \\
\hline$\ldots \ldots \ldots \ldots$ & 57,020 & 5,330 & 17,370 & 3.55 & 3.96 \\
\hline May $\ldots \ldots \ldots \ldots \ldots \ldots \ldots$ & 10,765 & 2,333 & 4,355 & .89 & 1.03 \\
\hline June $\ldots \ldots \ldots \ldots \ldots \ldots$ & 9,010 & 850 & 3,217 & .66 & .74 \\
\hline July $\ldots \ldots \ldots \ldots \ldots \ldots \ldots$ & 3,815 & 415 & 1,210 & .25 & .29 \\
\hline August $\ldots \ldots \ldots \ldots \ldots \ldots$ & 29,550 & 340 & 7,117 & 1.45 & 1.67 \\
\hline September $\ldots \ldots \ldots \ldots \ldots$ & 8,660 & 710 & 2,626 & .54 & .60 \\
\hline October $\ldots \ldots \ldots \ldots \ldots \ldots$ & 4,775 & 445 & 1,536 & .31 & .36 \\
\hline November $\ldots \ldots \ldots \ldots \ldots \ldots$ & 1,022 & 512 & 712 & .15 & .17 \\
\hline December $\ldots \ldots \ldots \ldots \ldots \ldots$ & 80,250 & 710 & 13,293 & 2.71 & 3.12 \\
\hline The year $\ldots \ldots \ldots \ldots \ldots \ldots$ & 108,375 & 340 & 8,454 & 1.73 & 23.35 \\
\hline
\end{tabular}

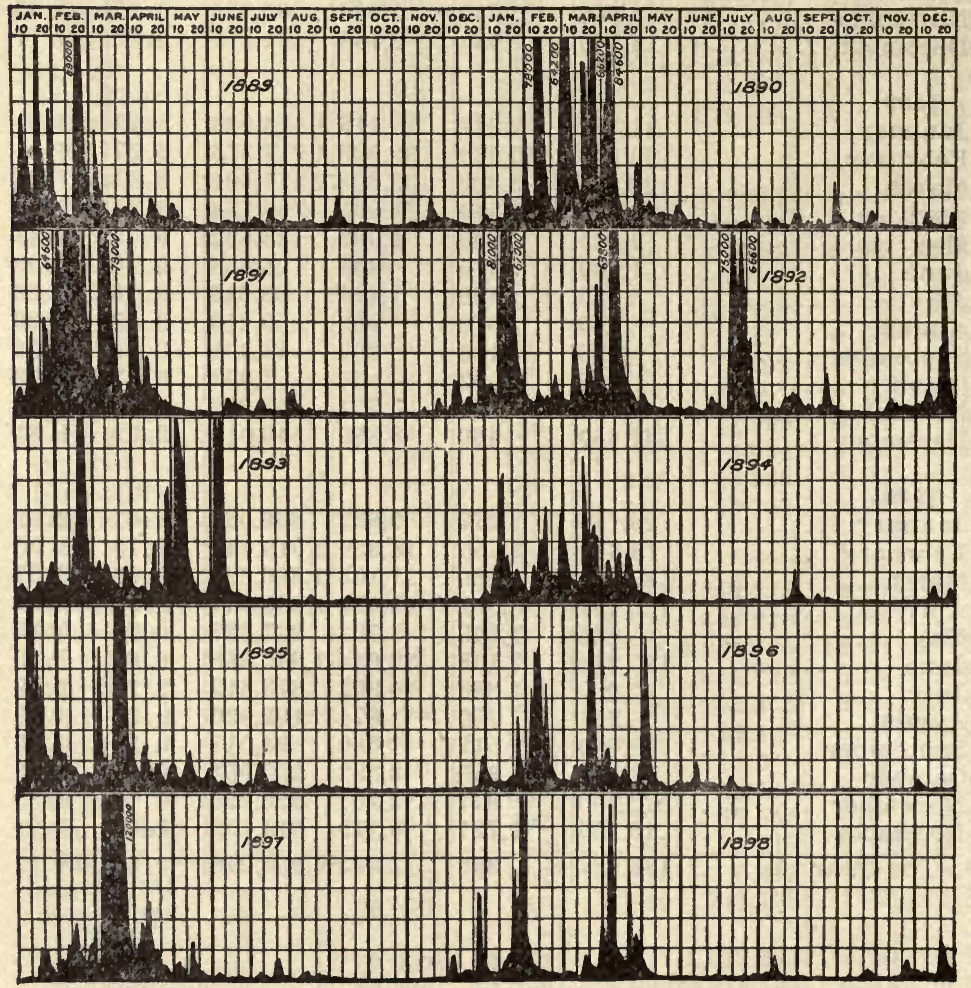

Fig. 14.-Discharge of Black Warrior River at Tuscaloosa, Alabama, 1889-1898. 


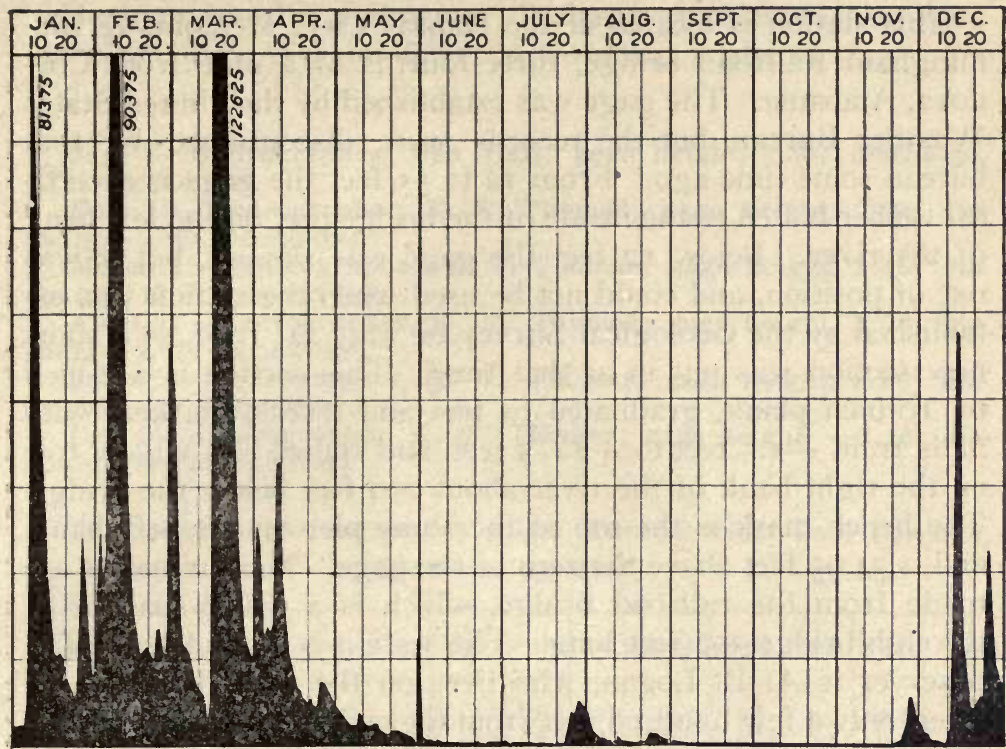

FIG., 15.-Discharge of Black Warrior R'iver at Tuscaloosa, Alabama, 1899.

Minimum monitily discharge of Black Warrior River at Tuscaloosa, Ala., with corresponding net horse power per foot of fall on a water wheel realizing 80 per cent. of the theoretical power.

[Drainage area, 4,900 square miles.]

\begin{tabular}{|c|c|c|c|c|c|c|c|c|c|}
\hline & \multicolumn{3}{|c|}{1899} & \multicolumn{3}{|c|}{1900} & \multicolumn{3}{|c|}{1901} \\
\hline & 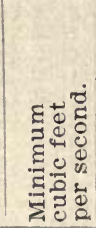 & 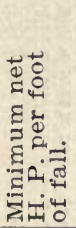 & 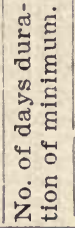 & 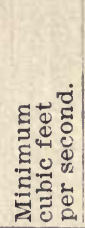 & 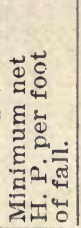 & 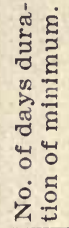 & 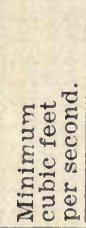 & 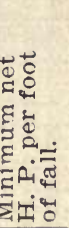 & 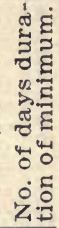 \\
\hline January & 2,222 & 202 & 1 & 2,277 & 207 & 1 & 4,370 & 398 & 1 \\
\hline February & 10,030 & 912 & 2 & 2,555 & 232 & 1 & 4,165 & 378 & 1 \\
\hline March ... & 5,330 & 485 & 1 & 8,282 & 753 & 1 & 3,815 & 347 & 1 \\
\hline April .... & 4,539 & 413 & 1 & 5,302 & 483 & 1 & 5,330 & 485 & 1 \\
\hline May $\ldots . .$. & 790 & 72 & 1 & 1,645 & 150 & 1 & 2,333 & 212 & 1 \\
\hline June & 175 & 16 & 1 & 2,139 & 194 & 1 & 850 & 77 & 2 \\
\hline July & 160 & 15 & 7 & 2,883 & 262 & 1 & 415 & 38 & 1 \\
\hline August $\ldots \ldots$ & 370 & 34 & 2 & 750 & 68 & 2 & 340 & 31 & 1 \\
\hline September ... & 110 & 10 & 1 & 512 & 47 & 1 & 710 & 65 & 1 \\
\hline October ...... & 92 & 9 & 1 & 355 & 32 & 1 & 445 & 40 & 1 \\
\hline November .... & 127 & 12 & 5 & 977 & 89 & 1 & 512 & 47 & 1 \\
\hline December .... & 460 & 42 & 1 & 2,721 & 247 & 1 & 710 & 65 & 1 \\
\hline
\end{tabular}


2. BLACK WARRIOR RIVER NEAF CORDOVA, ALABAMA.

This station is locater at the Kansas City, Memphis \& Birmingham Railroad bridge, three-fourths of a mile from Cordova, Alabama. The gage was established by the United States Weather Bureau, but the records were discontinued by that bureau some time ago. From 12 to 55 feet the gage is a vertical timber bolted to the inside of the bridge pier on the left bank of the river. Below 12 feet the gage was sloping, but it was out of position, and could not be used when the station was established by the Geological Survey on May 21, 1900, so a short new section was put in at that time. This section is a 2 -inch by Io-inch plank, graduated to feet and tenths, marked with nails from - I.5 feet to $+\mathrm{I} 2.5$ feet, and spiked to a willow tree on the right bank of the river about 200 feet below the bridge. The bench mark is the top of the stone pier on the left bank, and is 54.95 feet above the zero of the gage. Measurements are made from the railrolad bridge, which is a single-span, iron, through bridge 300 feet long. The section is a good one. The observer is A. B. Logan, who lives on the right bank of the river, only a few hundred feet from the end of the bridge. During 1900 measurement was made by Max Hall as follows :

Daily gage height, in feet, of Black Warrior River near Cordova, Alabama, for 1900 .

\begin{tabular}{|c|c|c|c|c|c|c|c|c|}
\hline Day & May. & June & July & Aug: & Sept. & Oct. & Nov. & Dec \\
\hline & & & & & & -0.8 & -0.1 & \\
\hline & & 2.8 & 5.2 & .5 & .2 & -.8 & $+.5 \mid$ & 1.0 \\
\hline & & $\begin{array}{l}5.3 \\
7.1\end{array}$ & $\begin{array}{l}7.1 \\
6.5\end{array}$ & .4 & .1 & $=.9$ & $\begin{array}{r}8 \\
4 \\
\end{array}$ & 1.8 \\
\hline & & 7.5 & 5.3 & .1 & -.1 & -1.0 & .3 & 1.9 \\
\hline . & & 7.6 & 4.6 & .0 & -.2 & -1.0 & .3 & 1.8 \\
\hline & & $\begin{array}{l}6.5 \\
7.3\end{array}$ & $\begin{array}{l}3.5 \\
2.1\end{array}$ & .12 & $=.3$ & $|-1.1|$ & .2 & $\begin{array}{l}1.4 \\
1.2\end{array}$ \\
\hline . & & 6.5 & 2.5 & .3 & -.5 & \begin{tabular}{|l|} 
\\
\end{tabular} & .1 & 1.0 \\
\hline 10 & & 4.5 & 2.7 & & -.5 & .9 & \begin{tabular}{|l|}
.0 \\
11
\end{tabular} & .8 \\
\hline & …... & $\begin{array}{l}3.2 \\
3.0\end{array}$ & 1.0 & .4 & $=.6$ & $\begin{array}{r}.1 \\
9\end{array}$ & $=.1$ & .7 \\
\hline & $\ldots \ldots$. & 4.6 & 1.0 & & -.7 & 9.2 & $=.24$ & .8 \\
\hline ............ & & 8.6 & .8 & .5 & & 3.2 & $-.3 \mid$ & .8 \\
\hline & & 9.8 & .5 & .6 & 1.5 & 2.3 & -.3 & .8 \\
\hline & & & & .6 & .9 & & -.3 & .6 \\
\hline$\ddot{\ddot{*}} \quad r$ & …... & 8.2 & $\begin{array}{l}4 \\
3 \\
\end{array}$ & .0 & .0 & 1.0 & I. $\cdot 4$ & .5 \\
\hline ........ & $\ldots \ldots$ & 13.4 & .3 & .1 & .0 & .3 & .2 & .4 \\
\hline$?$ & & 10.0 & 2 & .0 & -.1 & & .1 & .5 \\
\hline & 0.1 & 6.1 & & .2 & -.2 & .1 & & 1.5 \\
\hline & 1 & $\begin{array}{r}6.5 \\
15.2\end{array}$ & 1.0 & .3 & $=.3$ & .0 & 1.1 & 3.0 \\
\hline ..... & 1.5 & & .5 & .8 & -.4 & .7 & .8 & 5.0 \\
\hline 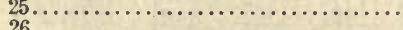 & 1.5 & 33.8 & .9 & 1.7 & -.4 & .5 & & $4 . n$ \\
\hline & 1.0 & 31.3 & .5 & .8 & -.5 & .3 & 5.1 & 3.1 \\
\hline & .6 & 22.1 & (1.6! & .2 & $=.5$ & .2 & $\begin{array}{l}4.9 ! \\
3.6 \\
\end{array}$ & 2.5 \\
\hline & .8 & 22.4 & & .1 & -.7 & 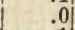 & 2.8 & 1.8 \\
\hline & .9 & 16.5 & & & -.7 & $-.1 \mid$ & 1.9 & 1.7 \\
\hline 31 . & .9 & & 1.5 & & & -.1 & $|\ldots . .|$. & 2.8 \\
\hline
\end{tabular}


During I90I the following discharge measurements were made on Black Warrior River at Cordova, Alabama: 1901.

Jan. 8-Hydrographer, Max Hall; gage height, 1.30; discharge, 1,781 second-feet.

Feb. 18-Hydrographer, K. T. Thomas; gage height, 2.40; discharge, 2,863 second-feet.

March 12-Hydrographer, K. T. Thomas; gage height, 9.45; discharge, 13,279 second-feet.

April 17-Hydrographer, K. T. Thomas; gage height, 1.70; discharge, 2,024 second-feet.

June 20-Hydrographer, K. T. Thomas; gage height, 0.00 ; discharge, 644 second-feet.

Oct. 26-Hydrographer, K. T. Thomas; gage height, -0.40 ; discharge, 385 second-feet.

Daily gage height, in feet, of Black Warrior River near Cordova, Alabama, for 1901.

\begin{tabular}{|c|c|c|c|c|c|c|c|c|c|c|c|c|}
\hline Day & Jan, & Feb. & Mar. & April & May & Jun. & July & Aug. & Sept. & Oct. & Nov.] & Dec \\
\hline & 3.7 & 4.09 & 1.00 & 5.40 & 1. & 1.6 & +0.1 & -0.6 & 0.7 & 1.2 & & \\
\hline & 3.1 & & 1.60 & & & 4.5 & 0.3 & & & 0.8 & $|-0.5|$ & 0.2 \\
\hline & 2.8 & $\begin{array}{r}4.50 \\
17.60\end{array}$ & $\begin{array}{l}2.30 \\
1.90\end{array}$ & $\begin{array}{l}14.50 \\
10.90\end{array}$ & 1. & 2.8 & 0.1 & -0.65 & 0.4 & 0.5 & $-0.5 \mid$ & 0.1 \\
\hline & 2.3 & 18.00 & $\begin{array}{l}1.60 \\
1.60\end{array}$ & 7.00 & 1. & 2.0 & $\begin{array}{r}0.0 \\
-0.2\end{array}$ & -0.70 & 0.1 & $\begin{array}{l}0.4 \\
0.3\end{array}$ & -0.4 & ${ }_{0.0}^{0.0}$ \\
\hline & 2.0 & 9.10 & 1.80 & 5.80 & 0. & 2.6 & -0.2 & -0.70 & & 0.2 & -0.4 & 0.1 \\
\hline & 1.7 & 5.6 & 1.50 & 4.90 & 0 . & 2.7 & -0.3 & -0.70 & -0.1 & 0.1 & -0.3 & 0.1 \\
\hline & 1.5 & 5.20 & 1.30 & 4.20 & 0. & 2.1 & -0.3 & -0.65 & -0.2 & 0.0 & $-0.3 \mid$ & 0.1 \\
\hline & $\begin{array}{l}1.3 \\
2.6\end{array}$ & 8.10 & $\begin{array}{l}1.00 \\
17.50\end{array}$ & $\begin{array}{l}3.50 \\
2.80\end{array}$ & 0. & 1.1 & 0.4 & -0.60 & -0.3 & -0.1 & & 0.1 \\
\hline & 199 & 6.20 & 20.50 & 2.30 & 0 & 2.2 & -0.6 & -0.60 & -05 & -2 & -0. & \\
\hline & & 5.40 & 10.80 & 2.10 & 0 . & 1.5 & -0.6 & -0.30 & & & -0.41 & \\
\hline & & 480 & 6.20 & 180 & 0. & 1.1 & -0.6 & +40 & & 00 & -0.0 & \\
\hline & & & 5.30 & 2.5 & 3. & 1.0 & & & & & -0. & \\
\hline & & 380 & 4.20 & 2.80 & 1. & 0.9 & -0.7 & 20 & & 0.6 & -0 & 6.2 \\
\hline 16 & & & 3.50 & 2.30 & 1. & 0.7 & -0 & 5.00 & & 3 & -0 & \\
\hline & & & & 1.80 & 0. & 0.6 & & 12.10 & & & -0 & \\
\hline & 4.30 & 2.60 & 2.20 & 2.20 & 0. & 0.5 & & 6.8 & & .0 & -0 & \\
\hline & 3.80 & & 1.90 & 9.40 & 0. & 0.3 & |+1.1 & 10.80 & & & -0 & \\
\hline & 3.10 & 2.00 & 2.40 & 16.10 & 1. & 0.2 & & 8.70 & & -0.2 & & \\
\hline & & 1.80 & 5.00 & 11.50 & 5. & 0.0 & & 8.50 & 1.1 & -0.2 & -0. & \\
\hline & & 1.60 & 4.20 & 6.80 & 7. & $3-0.1$ & 0.5 & 11.20 & 0.8 & -0.3 & -0. & 1. \\
\hline & & .30 & 3.50 & 5.40 & 5. & $5 \mid-0.2$ & 0.0 & 7.30 & 0.5 & -0.3 & 0. & 1. \\
\hline & 5. & 1.40 & 3.50 & 4.50 & 3. & $6 \mid=0.3$ & -0.2 & 5.20 & 0.4 & -0.4 & -0 & 2 \\
\hline & $\begin{array}{l}5.40 \\
5.00\end{array}$ & 1.20 & 11.20 & $\begin{array}{l}8.00 \\
3.00\end{array}$ & 2. & & -0.4 & $\begin{array}{l}2.00 \\
2.80\end{array}$ & 0.2 & -0.4 & -0.21 & 2 \\
\hline & & 10 & 6.90 & & 1. & & -0.5 & 2.00 & 0.1 & -0.4 & -0 & 2.6 \\
\hline & $\begin{array}{r}4.20 \\
3.80\end{array}$ & 1.00 & 5.30 & 2.20 & 1. & & -0.5 & 1.50 & 0.1 & -0.4 & $-0.1 \mid$ & 5.0 \\
\hline & & $\cdots$ & $\begin{array}{l}4.00 \\
4.00\end{array}$ & $\begin{array}{l}1.90 \\
1.90\end{array}$ & 1. & $0=0.5$ & & $\begin{array}{l}1.400 \\
1.20\end{array}$ & $\begin{array}{l}1.0 \\
2.3\end{array}$ & -0.5 & $=0.21$ & 20. \\
\hline & 4. & ... & 6.20 & $\ldots$ & 1. & & -0.6 & 1.00 & & -0.5 & ..... & 13.5 \\
\hline
\end{tabular}


Rating table for Black Warrior River at Cordova, Alabama, for 1900 and 1901.

\begin{tabular}{|c|c|c|c|c|c|c|c|}
\hline 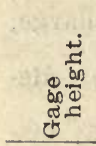 & 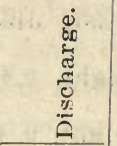 & 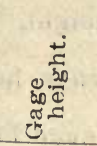 & 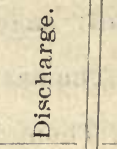 & 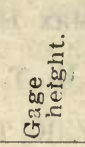 & 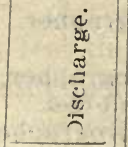 & 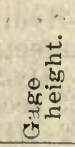 & 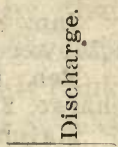 \\
\hline Feet. & Second $f t$. & Feet. & Sec. ft & Feet. & sicond fi. & Feet & Secoind $f t$. \\
\hline-0.7 & 205 & 3.3 & 4,144 & 1.3 & 10,064 & 11.3 & 15,984 \\
\hline-0.6 & 260 & 3.4 & 4,292 & 7.4 & 10,212 & 11.4 & 16,132 \\
\hline-0.5 & 320 & 3.5 & 4,440 & 7.5 & 10,360 & 11.5 & 16,280 \\
\hline-0.4 & 384 & 3.6 & 4,588 & 7.6 & 10,508 & 11.6 & 16,428 \\
\hline-0.3 & 450 & 3.7 & 4,736 & 7.7 & 10,656 & 11.7 & 16,576 \\
\hline-0.2 & 518 & 3.8 & 4,884 & 7.8 & 10,804 & 11.8 & 16,724 \\
\hline-0.1 & 588 & 3.9 & 5,032 & 7.9 & 10,952 & 11.9 & 16,872 \\
\hline 0.0 & 660 & 4.0 & 5,180 & 8.0 & 11,16 v & 12.0 & 17,020 \\
\hline 0.1 & 734 & 4.1 & 5,328 & 8.1 & 11,248 & 12.1 & 17,168 \\
\hline 0.2 & 81.0 & 4.2 & 5,476 & 8.2 & 11,396 & 12.2 & 17,316 \\
\hline 0.3 & 888 & 4.3 & 5,624 & 8.3 & 11,544 & 12.3 & 17,464 \\
\hline 0.4 & 968 & 4.4 & 5,772 & 8.4 & 11,692 & 12.4 & 17,612 \\
\hline 0.5 & 1,050 & 4.5 & 5,920 & 8.5 & $11,840^{\circ}$ & 12.5 & 17,760 \\
\hline 0.6 & 1,134 & 4.6 & 6,068 & 8.6 & 11,988 & 12.6 & 17,908 \\
\hline 0.7 & 1,220 & 4.7 & 6,216 & 8.7 & 12,136 & 12.7 & 18,056 \\
\hline 0.8 & 1,307 & 4.8 & 6,364 & 8.8 & 12,284 & 12.8 & 18,204 \\
\hline 0.9 & 1,396 & 4.9 & 6,512 & 8.9 & 12,432 & 12.9 & 18,352 \\
\hline 1.0 & 1,486 & 5.0 & 6,660 & 9.0 & 12,580 & 13.0 & 18,500 \\
\hline 1.1 & 1,577 & 5.1 & 6,808 & 9.1 & 12,728 & 13.1 & 18.648 \\
\hline 1.2 & 1,669 & 5.2 & 6,956 & 9.2 & 12,876 & 13.2 & 18,796 \\
\hline 1.3 & 1,762 & 5.3 & 7,104 & 9.3 & 13,024 & 13.3 & 18,944 \\
\hline 1.4 & 1,856 & 5.4 & 7,252 & 9.4 & 13,172 & 13.4 & 19,092 \\
\hline 1.5 & 1,951 & 5.5 & 7,400 & 9.5 & 13,320 & 13.5 & 19.240 \\
\hline 1.6 & 2,047 & 5.6 & 7,548 & 9.6 & 13,468 & 13.6 & 19,388 \\
\hline 1.7 & 2,144 & 5.7 & 7,696 & 9.7 & 13,616 & 13.7 & 19,536 \\
\hline 1.8 & 2,242 & 5.8 & $7,844 \mid$ & 9.8 & 13,764 & 13.8 & 19.684 \\
\hline 1.9 & 2,342 & 5.9 & 7,992 & 9.9 & 13,912 & 13.9 & 19,832 \\
\hline 2.0 & 2,444 & 6.0 & 8,140 & 10.0 & 14,060 & 14.0 & 19,980 \\
\hline 2.1 & 2,547 & 6.1 & 8,288 & 10.1 & 14,208 & 14.1 & 20,128 \\
\hline 2.2 & 2,652 & 6.2 & 8,436 & 10.2 & 14,356 & 14.2 & 20,276 \\
\hline 2.3 & 2,758 & 6.3 & 8,584 & 10.3 & 14,504 & 14.3 & 20,424 \\
\hline 2.4 & 2,868 & 6.4 & 8,732 & 10.4 & 14,652 & 14.4 & 20,572 \\
\hline 2.5 & $2,988 \mid$ & 6.5 & 8,880 & 10.5 & 14,800 & 14.5 & 20.720 \\
\hline 2.6 & 3,118 & 6.6 & $9,028 \mid$ & 10.6 & $14,948 \mid$ & 14.6 & 20868 \\
\hline 2.7 & 3,258 & 6.7 & 9.176 & 10.7 & 15,096 & 14.7 & 21.016 \\
\hline 2.8 & 3,404 & 6.8 & $9,324 \|$ & 10.8 & 15,244 & 14.8 & 21.164 \\
\hline 2.9 & 3,552 & 6.9 & $9,472||$ & 10.9 & 10,392 & 14.9 & 21,312 \\
\hline 3.0 & 3,700 & 7.0 & 9.620 & 11.0 & 15,540 & 15.0 & 21,460 \\
\hline 3.1 & 3,848 & 7.1 & $9,768 \mid 1$ & 11.1 & 15,688 & & \\
\hline 3.2 & 3,996 & 7.2 & $9,916 \|$ & 11.2 & 15,836 & & \\
\hline
\end{tabular}

Note.-This table applied to the foregoing "Daily gage heights" gives the cubic feet per second flowing in the river on each date for which the gage height is given. 
Estimated monthly discharge of Mulberry Fork of Black Warrior River, near Cordova, Alabama.

[Drainage area, 237 square miles.]

\begin{tabular}{|c|c|c|c|c|c|}
\hline \multirow[b]{2}{*}{ Month. } & \multicolumn{3}{|c|}{ Discharge in second-feet. } & \multicolumn{2}{|c|}{ Run-off. } \\
\hline & $\begin{array}{l}\text { Maxi- } \\
\text { mum. }\end{array}$ & $\begin{array}{l}\text { Mini- } \\
\text { mum. }\end{array}$ & Mean. & $\begin{array}{c}\text { Second- } \\
\text { feet per } \\
\text { square } \\
\text { mile. }\end{array}$ & $\begin{array}{l}\text { Depth } \\
\text { in } \\
\text { inches. }\end{array}$ \\
\hline 1900. & & & & & \\
\hline June . & 49,284 & 1,134 & 16,185 & 8.52 & 9.51 \\
\hline$\ldots \ldots \ldots \ldots$ & 11,248 & 810 & 2,975 & 1.57 & 1.81 \\
\hline August $\ldots \ldots \ldots \ldots$ & 2,144 & $660^{\dagger}$ & 1,016 & .53 & .61 \\
\hline September.$\ldots \ldots \ldots \ldots \ldots \ldots$ & 1,951 & 205 & 556 & .29 & .32 \\
\hline Octaber $\ldots . \ldots \ldots, \ldots, \ldots$ & 13,098 & $60^{\circ}$ & 1,732 & .91 & 1.05 \\
\hline November $\ldots \ldots \ldots$. & 6,808 & 384 & 1,487 & .78 & .87 \\
\hline December $\ldots \ldots \ldots \ldots \ldots$ & 6,660 & 968 & 2,154 & 1.13 & 1.30 \\
\hline 1901. & & & & & \\
\hline January ...... & 51,800 & 1,762 & 8,713 & 4.59 & 5.29 \\
\hline February $\ldots \ldots \ldots \ldots \ldots$ & 31,820 & 1,486 & 6,616 & 3.48 & 3.62 \\
\hline March $\ldots \ldots \ldots \ldots \ldots \ldots$ & 29,600 & 1,486 & 6,637 & 3.49 & 4.02 \\
\hline April $\ldots \ldots \ldots \ldots \ldots \ldots \ldots$ & 23,088 & 2,242 & 6,967 & 3.67 & 4.10 \\
\hline May $\ldots \ldots \ldots \ldots \ldots \ldots \ldots$ & 10,064 & 968 & 2,539 & 1.34 & 1.54 \\
\hline June $\ldots \ldots \ldots \ldots \ldots \ldots$ & 5,920 & 320 & 1,582 & .83 & .93 \\
\hline July $\ldots \ldots \ldots \ldots$ & 3,404 & 205 & 631 & .33 & .38 \\
\hline August $\ldots \ldots \ldots \ldots$. & 17,168 & 205 & 4,155 & 2.19 & 2.53 \\
\hline September.$\ldots \ldots \ldots \ldots$ & 6,660 & 320 & 1,415 & .74 & .83 \\
\hline October $\ldots \ldots \ldots \ldots \ldots \ldots$ & 1,669 & 320 & 687 & .36 & .42 \\
\hline November $\ldots \ldots \ldots \ldots \ldots$ & 660 & 320 & 468 & .25 & .28 \\
\hline December $\ldots \ldots \ldots \ldots \ldots \ldots$ & 31,820 & 518 & 4,923 & 2.59 & 2.99 \\
\hline The year $\ldots \ldots \ldots \ldots \ldots$ & 51,800 & 205 & 3,778 & 1.99 & 26.93 \\
\hline
\end{tabular}


Minimum monthly discharge of Black Warrior River at Cordova, Ala., with corresponding net horse power per foot of fall on a water wheel realizing 80 per cent. of the theoretical power.

[Drainage area, 237 square miles.]

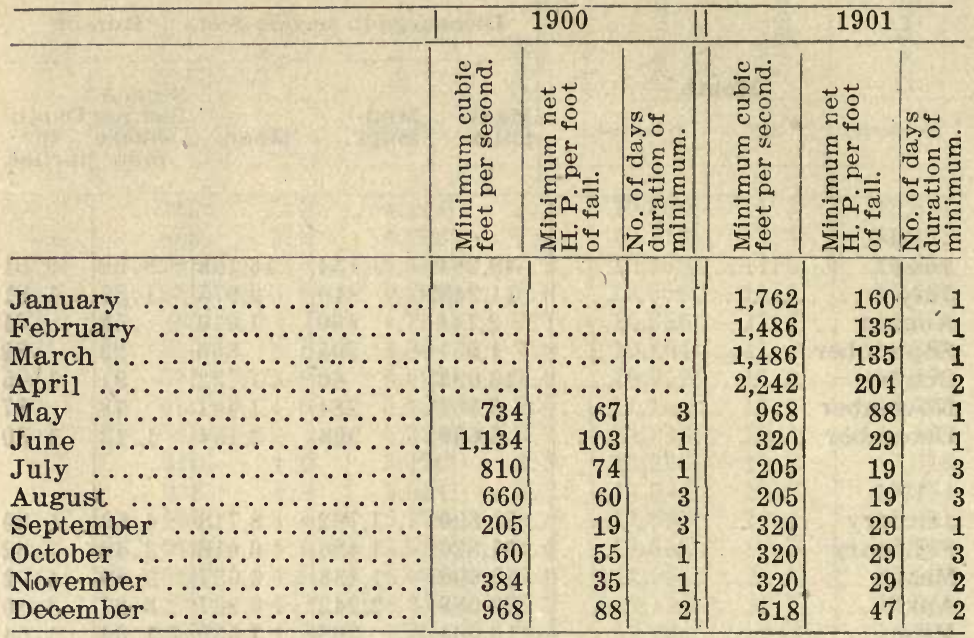

NoTE.-To find the minimum net horse power available at a shoal on this stream, near this station, for any month, multiply the total fall of the shoal by the "Net H. P. per foot of fall" in this table for that month.

\section{SURVEY OF BLACK WARRIOR RIVER, ALABAMA.}

The Black Warrior River is formed by the junction of the Mulberry and Sipsey forks of Black Warrior at Old Warrior Town in Walker County, Alabama, and runs in a southwesterly direction past Tuscaloosa to Demopolis, Ala., at which point it enters the Tombigbee River. Above Tuscaloosa it is known as the Black Warrior River, and below Tuscaloosa as the Warrior River.

The accompanying profile is made from the surveys of the Corps of Engineers, U. S. A.

A great deal of work is being done by the Government on this river in order to make it navigable as an outlet to important coal fields above.

In the 92 miles from Old Warrior Town to Tuscaloosa, there is a fall of 158 feet. The distribution of this fall is shown by the following table, giving distances in miles above Tuscaloosa and elevations of water surface above sea level. 


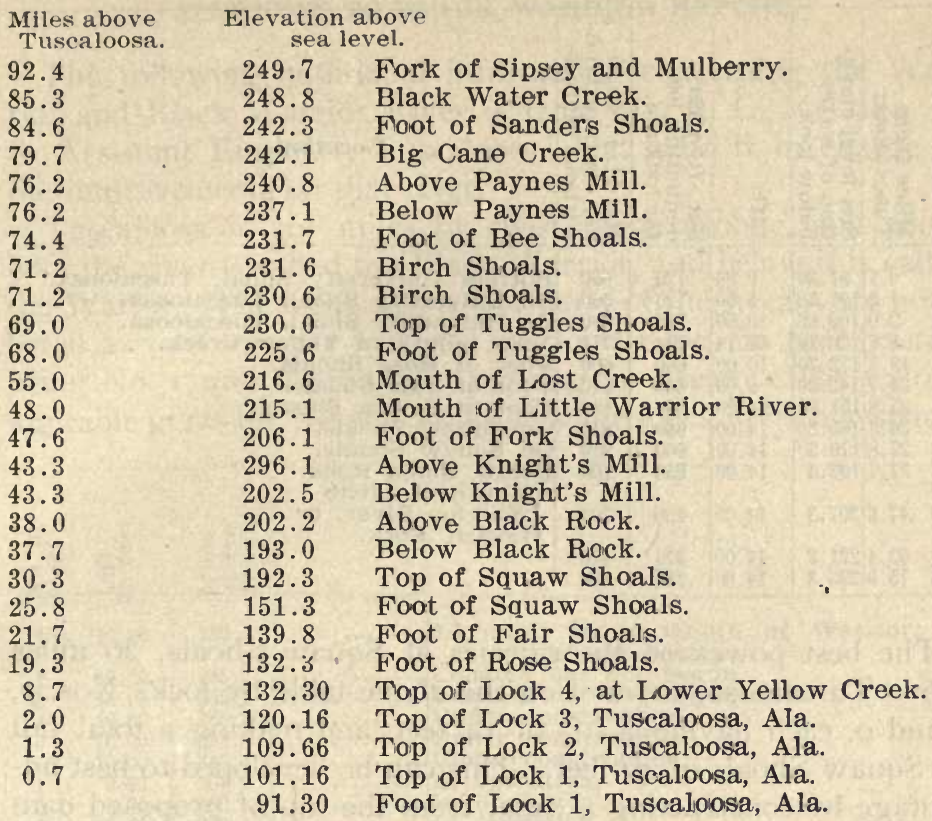

There are gage stations, both at Tuscaloosa and Cordova, Ala., where systematic discharge measurements have been made, the results of which are given in the foregoing pages. Comparative measurements at the two stations at same stage, which was same stage as low water in November, I90I, shows a discharge of 825 second-feet at Tuscaloosa, and 285 second-feet at Cordova. At minimum stage of dryest years the water gets considerably lower, as is shown by the records referred to, but the figures named are safe for low season in all ordinary years, and will be used in this discussion, for determining the power available at different sites along the river.

The locks and proposed locks on this section of the river begin with No. I, at Tuscaloosa, and are numbered up the river. Locks I, 2, 3 and 4 are about completed, and others are projected, but the locations of the latter in the following list are approximated. However, the exact location of each is immaterial in showing the power available. The following is a table showing positions of locks and lock sites in miles above Tuscaloosa, the sea level elevation of water below each, the lift at each and the net horsepower that can be developed at each day on an 80 per cent. turbine during dry season in ordinary years, like I900, after deducting 100 second-feet for lockage. 


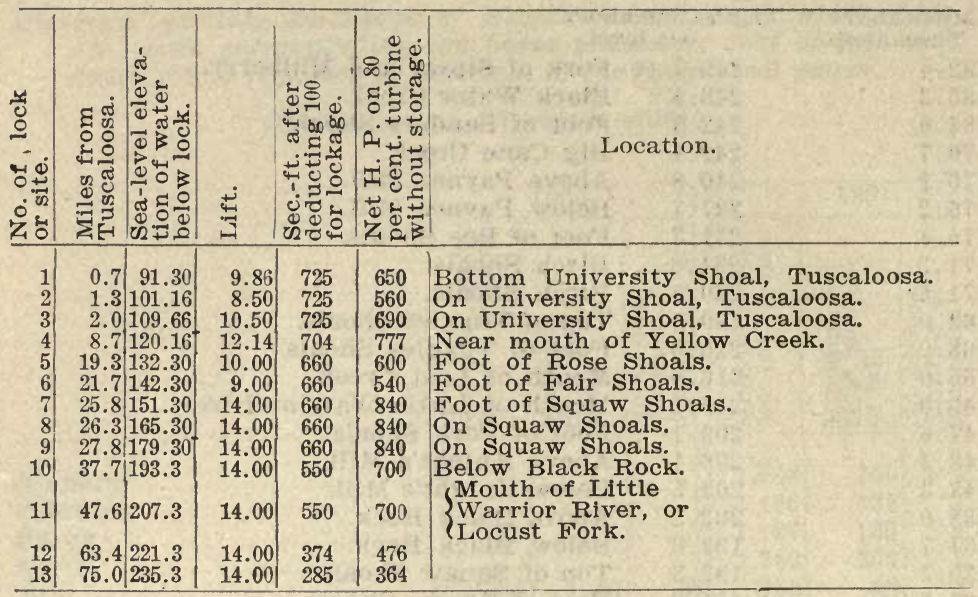

The best power on the river is at Squaw Shoals, 26 miles above Tuscaloosa, covered on the above table by locks Nos. 7 , 8 and 9 , each having a lift of $\mathrm{I}_{4}$ feet, and making a total fall on Squaw Shoals of 42 feet. This can be developed to best advantage by constructing a canal from the top of proposed dam at Lock No. 9, along the river bank, two miles in length, to a point opposite the foot of Squaw Shoals, below Lock No. 7 . This canal taking the river water not need for lockage, and allowing two feet for grade and storage, will utilize a net head of 40 feet, and produce 2,400 net horse power continuously, or 4,800 net horse power for a I2-hour run per day, storing the water above Lock No. 9 during the 12 idle hours.

It is to be remembered that the above estimates of power are for low season during ordinary years. There will be exceptional periods of minimum water in extremely dry years in which the entire flow of the river will be as low as Ioo secondfeet, and will, therefore, barely suffice for lockage during a busy season of boating on the river. See Nineteenth Annual Report, United States Geological Survey, Part IV, page 25I. But such seasons are rare, and the facilities for water transportation should compensate for them to a great extent. It is admitted that the cheapness of coal along this river would naturally make the water powers less valuable, but the cheapness of development in connection with Government dams would partly offset the cheapness of coal. It is believed that the proposed development at Squaw Shoals could be made at a very moderate cost, and that such an investment would pay handsomely. 


\section{APPENDIX TO BLACK WARRIOR REPORT.}

The following additional information concerning the Warrior and Black Warrior River is from Mr. R. C. McCalla, U. S. Assistant Engineer, Tuscaloosa, Ala., who is in charge of the improvements on that river.

Tuscaloosa is 361 miles by river above Mobile, and above here the river is called the Black Warrior, and below it is called the Warior. The locks on the two parts of the stream are numbered as two separate systems, the lowest lock in each system being No. I, and the numbers running up stream. The following table gives the lift and location of the locks in both systems:

\begin{tabular}{|c|c|c|c|c|}
\hline $\begin{array}{l}\overrightarrow{0} \\
\dot{0} \cdot \dot{0} \\
z \\
z\end{array}$ & 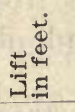 & 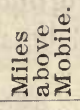 & & \\
\hline 1 & 10.00 & 230.5 & .......... 0.5 miles below mouth of & Warrior; lo- \\
\hline $\begin{array}{l}2 \\
3 \\
4 \\
5 \\
5 \\
6 \\
1 \\
2 \\
3 \\
4\end{array}$ & $\begin{array}{r}10.00 \\
10.00 \\
10.00 \\
10.00 \\
10.00 \\
9.86 \\
8.50 \\
10.50 \\
12.14\end{array}$ & $\begin{array}{l}246.2 \\
266.7 \\
282.3 \\
298.3 \\
315.2 \\
361.9 \\
362.3 \\
363.1 \\
370.1\end{array}$ & 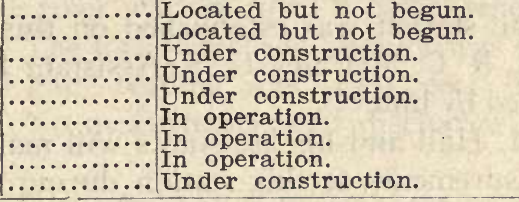 & \\
\hline
\end{tabular}

Between Lock No. 4 and the junction of Mulberry and Locust Forks, 407.8 miles above Mobile, there are projected seven locks at 14 feet lift each, but none of these are yet located. The following table gives the location, etc., of gages now established and read daily at 7 A. M. :

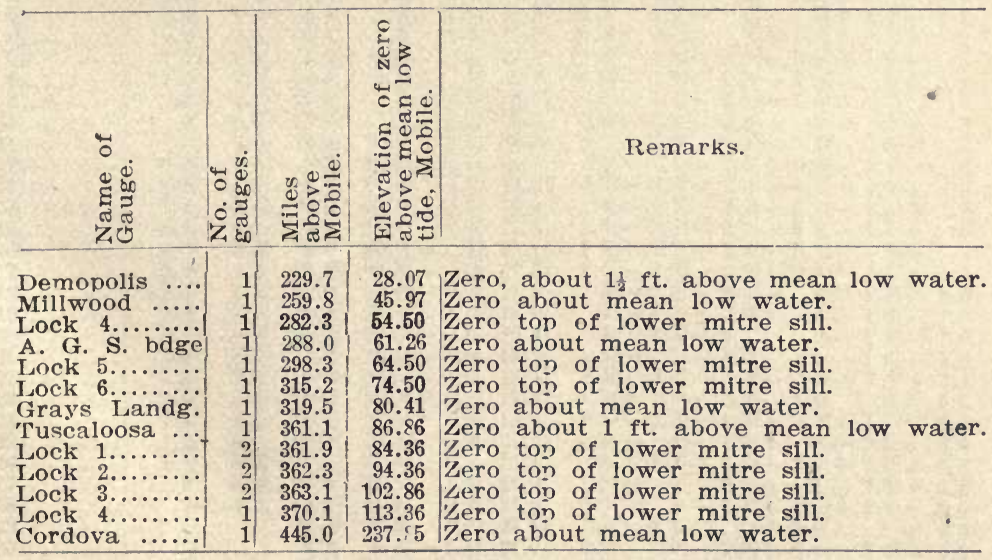


5. BLACK WARRIOR RIVER TRIBUTARIES.

At Clear Creek Falls, in Winston County, within a distance of half a mile, there is a fall of over 100 feet, distributed as follows :

Rapids above Upper Falls in 100 yards.......... 6 feet fall

Upper Falls, about ...................... 45 feet fall

Still pool for 275 yards $\ldots \ldots \ldots \ldots \ldots \ldots \ldots \ldots \ldots$. 00 feet fall

Lower Falls ........................... 27 feet fall

Rapids below Lower Falls .................... 30 feet fall

No discharge measurements have ever been made on this stream. It is thought best not to attempt to approximate its flow by any water-shed rule, as the stream originates from big springs. Actual discharge measurements will be made during 1902.

Little Warrior, or Locust Fork, is an important stream on which no surveys have been made. On December I, I90I, a hydrographic station was established on this stream at Palos, Ala., by Mr. R. C. McCalla, U. S. Assistant Engineer, and will be maintained by him.

Mr. B. M. Hall and his assistants will make a series of discharge measurements at this station during 1902. The only measurement so far was on January I8, 1902. The gage stood at 0.85 , and the discharge was 849 second-feet.

There are other tributaries having shoals that can be developed, but they have not been examined. 


\section{CHAPTER VI.}

\section{TOMBIGBEE RIVER AT COLLMBUS, MISSISSIPPI.}

This station is located about 1,000 feet below the highway bridge, $\mathrm{I} / 2$ miles from the Southern Railway depot at Columbus. The rod, which is in three sections, is fastened vertically to the rock bluff on the left bank. It is a 3 -inch by IO-inch pine timber 45 feet long, marked with brass figures and copper nails, the graduation extending fr $\mathrm{cm}-5.0$ feet to +40.0 feet. The initial point of sounding is the end of the iron bridge, right bank, downstream side. Bench mark No. I is 250 feet from the initial point of sounding. The bridge floor is 40.85 feet above the zero of the rod, and the top of the iron girder under the floor timbers is 39.85 feet above the zero. Bench mark No. 2 is the top of the rail at the depot of the Southern Railway, and is 55.2 feet above gage datum and I90.9 feet above mean sea level. The width of the river at low water is 160 feet. The maximum record height of the river was on April 8, 1892, when the gage registered 42 feet. The lowest recorded height was on October 26 , 1893 , when the gage reading was -3.9 feet. The danger line is at 33 feet. No measurements of discharge were made during 1900.

Daily gage height in feet of Tombigbee River at Columbus, Miss., for $19(1)$.

\begin{tabular}{|c|c|c|c|c|c|c|c|c|c|c|c|c|}
\hline Day & Jan, & Feb. & Mar. & April & May & Jun. & July & Aug. & Sept. & Oct. & Nov. & Dec \\
\hline & & & & & & & 9.7 & & & -2. & & \\
\hline & & & & & 6.5 & 8.0 & 18.3 & 4.0 & & -2 & & \\
\hline & $\begin{array}{l}8 \\
6 \\
\end{array}$ & & $\begin{array}{l}5.6 \\
4.4\end{array}$ & $\begin{array}{l}2.7 \\
1.9\end{array}$ & $\begin{array}{l}5.9 \\
4.5 \\
\end{array}$ & $\begin{array}{l}10.0 \\
13.4\end{array}$ & $\begin{array}{l}17.6 \\
16.2\end{array}$ & $\begin{array}{l}2.8 \\
1.9\end{array}$ & $=1.0$ & $=3.0$ & 5.5 & 1.9 \\
\hline & & 1.8 & & 1.5 & 2.8 & 15.3 & 15.9 & 1.0 & & -3.3 & 4.4 & 1.0 \\
\hline & & 3.5 & 3.4 & 1.3 & 1.5 & 17.0 & 15.4 & & & & & \\
\hline & & & & 1.3 & & 20.7 & 14.5 & & .3 & 3. & & \\
\hline & 1.0 & 3.3 & 14.4 & 1.1 & .5 & 23.6 & 13.5 & -.9 & & -2.2 & $2+$ & \\
\hline & 9 & $\begin{array}{l}4.2 \\
8.4\end{array}$ & $\begin{array}{l}15.1 \\
13.8\end{array}$ & .7 & 1.4 & $\begin{array}{l}25.5 \\
25.0\end{array}$ & $\begin{array}{r}10.0 \\
6.8\end{array}$ & -1.4 & 2.1 & & .1 & \\
\hline & & 7.8 & 11. & 11.7 & 3.3 & 23.6 & 5.5 & 2.2 & & & & \\
\hline & 6. & 7.6 & & & 3.6 & 21. & 4.0 & 2.6 & & +2.6 & -1.1 & \\
\hline & & 10.2 & & & 2.8 & 20. & 3.5 & 2.6 & & & & \\
\hline & & $\begin{array}{l}9.8 \\
8.1\end{array}$ & & & 2.2 & 18. & 2.0 & -2.7 & & & & \\
\hline & & & & 20.8 & 1.6 & 11. & 1.5 & $\frac{2.2}{9}$ & & b. & & \\
\hline & 2.8 & $\begin{array}{l}3.8 \\
4.6\end{array}$ & & $\begin{array}{l}20.09 \\
22.9\end{array}$ & .0 & 17. & 1.0 & -2.3 & & +0.4 & & \\
\hline & 2. & 3.8 & 4.6 & 26.9 & -.4 & 17.8 & .5 & -2.3 & 3. & & $\mid-1$ & 1. \\
\hline & & 3.2 & 9.4 & 27.6 & -1.0 & 18. & . & -1.8 & & & $4 \mid-1.6$ & \\
\hline & 2. & 2.8 & 15.6 & 27.5 & .8 & 16. & & -1.4 & $\mid-3.1$ & & & \\
\hline & & 3.5 & 18. & 27.1 & .5 & 15. & 1.3 & -1.1 & & -1.1 & & \\
\hline & & & & & & & & -1. & & & & \\
\hline & & o. & & & , & & 1.3 & -1.9 & & & & \\
\hline & & & & & & & 1.4 & -2.0 & & & & \\
\hline & & & & & & & 1 & 7.1 & & & & \\
\hline & 1.1 & & 11 & & & & (.J & $1+1.3$ & & & & \\
\hline & .9 & & & & ?. & & 5.8 & & -2.2 & & & \\
\hline &. & 4.2 & & & & & 7.21 & -1.1 & & & & \\
\hline & .5 & ... & & 9.4 & 1.0 & 21.7 & 5.9 & -1.0 & $|-2.5|$ & $\begin{array}{r}0 \\
+4\end{array}$ & & +4 \\
\hline & .4 &.. & & & .8 & .... & 6.7 & & $|-2.5|$ & +4.0 & $9+3.8$ & +4.8 \\
\hline
\end{tabular}


The following discharge measurements were made during I90I by K. T. Thomas:

March 11-Gage height, 12.33 feet; discharge, 19,425 second-feet. April 16-Gage height, 1.10 feet; discharge, 3,926 second-feet. June 25-Gage height, -2.50; discharge, 698 second-feet.

Oct. 30 - Gage height, -3.00; discharge, 657 second-feet.

Daily gage height in feet of Tombigbee River at Columbus, Miss., for 19() 1$.

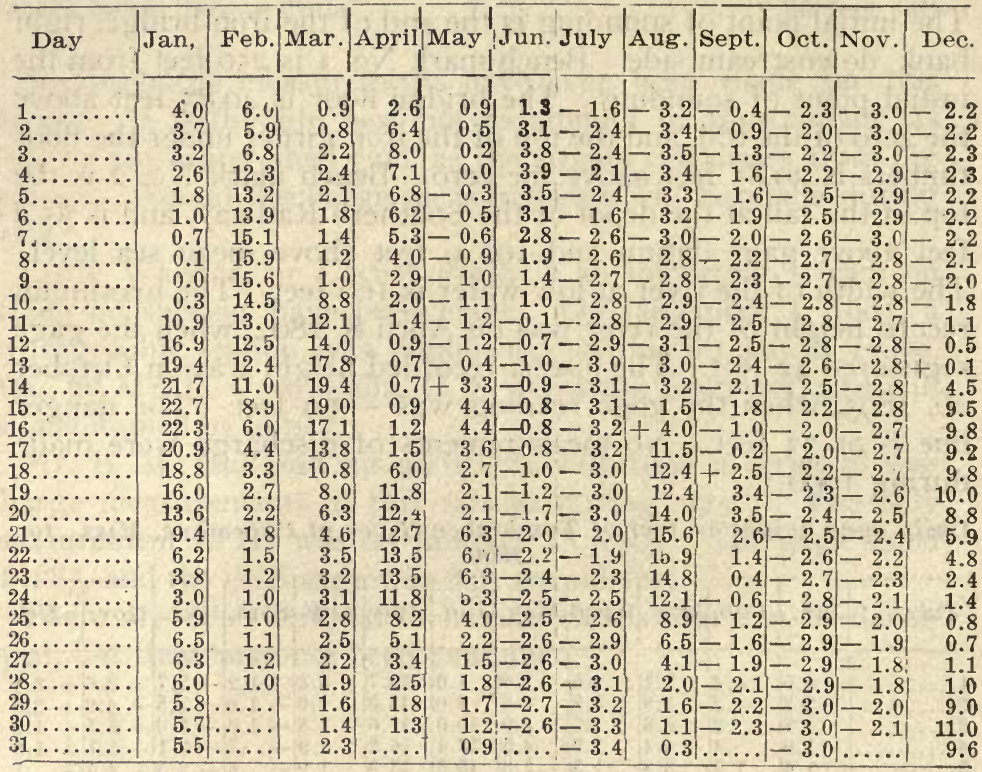


Rating table for Tombigbee River at Columbus, Miss., for 1900 and 191)1.

\begin{tabular}{|c|c|c|c|c|c|c|c|}
\hline 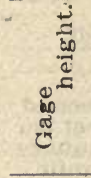 & 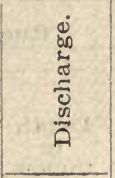 & 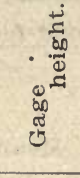 & 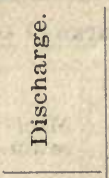 & & 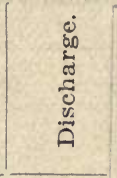 & 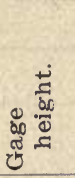 & 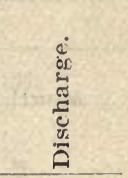 \\
\hline Feet. & Second $f t$. & Ficet. & secont:. & Feet. & secund ft. & Feet. & Second ft. \\
\hline-3.0 & 650 & 1.0 & 3,790 & 5.0 & 9,310 & 9.0 & 14,830 \\
\hline-2.9 & 668 & 1.1 & 3,928 & 5.1 & 9,448 & 9.1 & 14,968 \\
\hline-2.8 & 688 & 1.2 & 4,066 & 5.2 & 9,586 & 9.2 & 15,106 \\
\hline-2.7 & 712 & 1.3 & 4,204 & 5.3 & 9,724 & 9.3 & 15,244 \\
\hline-2.6 & 736 & 1.4 & 4,342 & 5.4 & 9,862 & 9.4 & 15,382 \\
\hline-2.5 & 752 & 1.5 & 4,480 & 5.5 & 10,000 & 9. & 15,520 \\
\hline-2.4 & 780 & 1.6 & 4,618 & 5.6 & 10,138 & 9.6 & 15,658 \\
\hline-2.3 & 810 & 1.7 & 4,756 & 5.7 & 10,276 & 9.7 & 15,796 \\
\hline-2.2 & 842 & 1.8 & 4,894 & 5.8 & 10,414 & 9.8 & 15,934 \\
\hline-2.1 & 877 & 1.9 & 5,032 & 5.9 & 10,552 & 9.9 & 16,072 \\
\hline-2.0 & 915 & 2.0 & 5,170 & 6.0 & 10,690 & 10.0 & 16,210 \\
\hline-1.9 & 956 & 2.1 & 5,308 & 6.1 & 10,828 & 10.1 & 16,348 \\
\hline-1.8 & 1,000 & 2.2 & 5,446 & 6.2 & 10,966 & 10.2 & 16,486 \\
\hline-1.7 & 1,047 & 2.3 & 5,584 & 6.3 & 11,104 & 10.3 & 16,624 \\
\hline-1.6 & 1,097 & 2.4 & 5,722 & 6.4 & 11,242 & 10.4 & 16,762 \\
\hline-1.5 & 1,150 & 2.5 & 5,860 & 6.5 & 11,380 & 10.5 & 16,900 \\
\hline-1.4 & 1,206 & 2.6 & 5,998 & 6.6 & 11,518 & 10.6 & 17,038 \\
\hline-1.3 & 1,265 & 2.7 & 6,136 & 6.7 & 11,656 & 10.7 & 17,176 \\
\hline-1.2 & 1,328 & 2.8 & 6,274 & 6.8 & 11,794 & 10.8 & 17,314 \\
\hline-1.1 & 1,394 & 2.9 & 6,412 & 6.9 & 11,932 & 10.9 & 17,452 \\
\hline-1.0 & 1,464 & 3.0 & 6,550 & 7.0 & 12,070 & 11.0 & 17,590 \\
\hline-0.9 & 1,537 & 3.1 & 6,688 & 7.1 & 12,208 & 11.5 & 18,280 \\
\hline-0.8 & 1,613 & 3.2 & 6,826 & 7.2 & 12,346 & 12.0 & 18,970 \\
\hline-0.7 & 1,692 & 3.3 & 6,964 & 7.3 & 12,484 & 12.5 & 19,660 \\
\hline-0.6 & 1,775 & 3.4 & 7,102 & 7.4 & 12,622 & 13. & 20,350 \\
\hline-0.5 & 1,863 & 3.5 & 7,240 & 7.5 & 12,760 & 13.5 & 21,040 \\
\hline-0.4 & 1,957 & 3.6 & 7,378 & 7.6 & 12,898 & 14.0 & 21,730 \\
\hline-0.3 & 2,057 & 3.7 & 7,516 & 7.7 & 13,036 & 14. & 22,420 \\
\hline-0.2 & 2,165 & 3.8 & 7,654 & 7.8 & 13,174 & 15. & 23,110 \\
\hline-0.1 & 2,283 & 3.9 & 7,792 & 7.9 & 13,312 & 15.5 & 23,800 \\
\hline 0.0 & 2,410 & 4.0 & 7,930 & 8.0 & 13,450 & 16. & 24,490 \\
\hline 0.1 & 2,548 & 4.1 & 8,068 & 8.1 & 13,588 & 16.5 & 25,180 \\
\hline 0.2 & 2,686 & 4.2 & 8,206 & 8.2 & 13,726 & 17.0 & 25,870 \\
\hline 0.3 & 2,824 & 4.3 & 8,344 & 8.3 & 13,864 & 17.5 & 26,560 \\
\hline 0.4 & 2,962 & 4.4 & 8,482 & 8.4 & 14,002 & 18.0 & 27,250 \\
\hline 0.5 & 3,100 & 4.5 & 8,620 & 8.5 & 14,140 & 18.5 & 27,940 \\
\hline 0.6 & 3,238 & 4.6 & 8,758 & 8.6 & 14,278 & 19,0 & 28,630 \\
\hline 0.7 & 3,376 & 4.7 & 8,896 & 8.7 & 14,416 & 19.5 & 29,320 \\
\hline 0.8 & 3,514 & 4.8 & 9,034 & 8.8 & 14,554 & 20.0 & 30,010 \\
\hline 0.9 & 3,652 & 4.9 & 9,172 & 8.9 & 14,692 & & \\
\hline
\end{tabular}


Estimated monthly discharge of Tombigbee River at Columbus, Miss. [Drainage area, 4,440 square miles.]

\begin{tabular}{|c|c|c|c|c|c|}
\hline \multirow[b]{2}{*}{ Month } & \multicolumn{3}{|c|}{ Discharge in second-feet. } & \multicolumn{2}{|c|}{ Run-off. } \\
\hline & $\begin{array}{l}\text { Maxi- } \\
\text { mum. }\end{array}$ & $\begin{array}{l}\text { Mini- } \\
\text { mum. }\end{array}$ & Mean. & $\begin{array}{l}\text { Depth } \\
\text { in } \\
\text { inches. }\end{array}$ & $\begin{array}{l}\text { Second } \\
\text { feet per } \\
\text { square } \\
\text { mile. }\end{array}$ \\
\hline 1900 & & & & & \\
\hline January . & 13,864 & 2,962 & 5,588 & 1.26 & 1.45 \\
\hline February & 16,486 & 2,686 & 8,659 & 1.95 & 2.03 \\
\hline March ... & 23,938 & 6,688 & 15,285 & 3.42 & 3.85 \\
\hline April $\ldots \ldots \ldots \ldots \ldots \ldots$ & 40,498 & 3,100 & 21,265 & 4.79 & 5.34 \\
\hline May $\ldots \ldots \ldots \ldots \ldots \ldots \ldots$ & 13,450 & 1,464 & 4,944 & 1.11 & 1.28 \\
\hline June $\ldots \ldots \ldots \ldots \ldots \ldots \ldots$ & 37,600 & 37,90 & 27,692 & 6.24 & 6.96 \\
\hline July $\ldots \ldots \ldots \ldots \ldots \ldots$ & 29,596 & 2,410 & 11,411 & 2.57 & 2.97 \\
\hline August...$\ldots \ldots \ldots \ldots \ldots$ & 10,414 & 707 & 2,257 & .51 & .59 \\
\hline September $\ldots \ldots \ldots \ldots \ldots \ldots$ & 1,775 & 632 & 950 & .21 & .23 \\
\hline October $\ldots \ldots \ldots \ldots \ldots \ldots$ & 10,138 & 566 & 3,989 & .90 & 1.04 \\
\hline November $\ldots \ldots \ldots \ldots \ldots$ & 10,000 & 1,097 & 4,304 & .97 & 1.08 \\
\hline December ................ & 11,794 & 1,464 & 5,239 & 1.18 & 1.36 \\
\hline The year $\ldots \ldots \ldots \ldots \ldots$ & 40,498 & 566 & 9,299 & 2.09 & 28.18 \\
\hline 1901. & & & & & \\
\hline January . & 33,736 & 2,410 & 14,193 & 3.20 & 3.69 \\
\hline February $\ldots \ldots \ldots \ldots \ldots$ & 24,352 & 3,790 & 12,533 & 2.83 & 2.95 \\
\hline March $\ldots \ldots \ldots \ldots \ldots \ldots$ & 29,182 & 3,514 & 10,884 & 2.45 & 2.33 \\
\hline April .................. & 21,040 & 3,376 & 9,890 & 2.23 & 2.49 \\
\hline May $\ldots \ldots \ldots \ldots \ldots \ldots \ldots$ & 11,656 & 1,328 & 4,949 & 1.11 & 1.28 \\
\hline June $\ldots \ldots \ldots \ldots \ldots \ldots \ldots$ & 7,792 & 707 & 2,767 & .62 & .69 \\
\hline July $\ldots \ldots \ldots \ldots \ldots \ldots$ & 10,97 & 582 & 730 & .16 & .18 \\
\hline August $\ldots \ldots \ldots \ldots \ldots \ldots \ldots$ & 24,352 & 582 & 7,673 & 1.73 & 1.99 \\
\hline September $\ldots \ldots \ldots \ldots \ldots \ldots$ & 7,240 & 753 & 2,008 & .45 & .50 \\
\hline October $\ldots \ldots \ldots \ldots \ldots \ldots$ & 915 & 650 & 748 & .17 & .20 \\
\hline November $\ldots \ldots \ldots \ldots \ldots$ & 1,000 & $650^{\circ}$ & 756 & .17 & .19 \\
\hline December .............. & 17,590 & 810 & 6,730 & 1.52 & 1.75 \\
\hline The year $\ldots \ldots \ldots \ldots \ldots$ & 33,736 & 582 & 6,155 & 1.39 & 18.74 \\
\hline
\end{tabular}


Minimum monthly discharge of Tombigbee River at Columbus, Miss., with corresponding net horse power per foot of fall on a water wheel realizing 80 per cent. of the theoretical power. [Drainage area, 4,440 square miles.]

\begin{tabular}{|c|c|c|c|c|c|c|}
\hline & \multicolumn{3}{|c|}{1900} & \multicolumn{3}{|c|}{1901} \\
\hline & 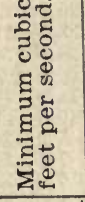 & 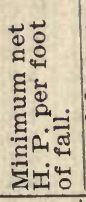 & 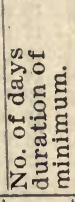 & 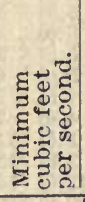 & 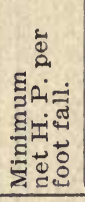 & 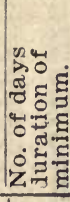 \\
\hline January . & 2,962 & 269 & 1 & 2,410 & 219 & 1 \\
\hline February ... & 2,686 & 244 & 1 & 3,790 & 345 & \\
\hline March ......... & 6,688 & 608 & 1 & 3,514 & 319 & 1 \\
\hline April .......... & 3,100 & 282 & 1 & 3,376 & 307 & 2 \\
\hline May $\ldots \ldots \ldots \ldots \ldots$ & 1,464 & 133 & 1 & 1,328 & 121 & 2 \\
\hline 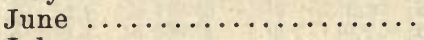 & 3,790 & 345 & 1 & 707 & 65 & 1 \\
\hline July $\ldots \ldots \ldots \ldots \ldots \ldots \ldots$ & 2,410 & 219 & 1 & 582 & 53 & 1 \\
\hline August $\ldots \ldots \ldots \ldots \ldots \ldots \ldots$ & 707 & 65 & 1 & 582 & 53 & 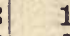 \\
\hline September $\ldots \ldots \ldots \ldots \ldots \ldots$ & 632 & 57 & 1 & 753 & 68 & 2 \\
\hline October $\ldots \ldots \ldots \ldots \ldots \ldots$ & 566 & 51 & 2 & 650 & 59 & 3 \\
\hline November $\ldots \ldots \ldots \ldots \ldots \ldots$ & 1,097 & 100 & 4 & 650 & 59 & 4 \\
\hline December $\ldots \ldots \ldots \ldots \ldots \ldots$ & 1,464 & 133 & 4 & 810 & 74 & 2 \\
\hline
\end{tabular}

NoTE.-To find the minimum net horse power available at a shoal on this stream, near this station, for any month, multiply the tow fall of the shoal by the "Net H. P. per foot of fall" in this table for that month.

\section{TOMBIGBEE RIVER NEAR EPES, ALABAMA.}

A record of gage heights has been kept at this station for the last ten years by the Alabama Great Southern Railway Company. The gage is painted on the center brick pier of the railway bridge of that company across the Tombigbee a half mile east of Epes, and is referred to two bench marks, the first, the top of the iron girder at the third cross-beam at the station, 8o feet from the right-bank end of the iron bridge, is 64.70 feet above datum of gage; the second, the top of the cross-tie or the base of the rail at the station, 80 feet from the right-bank end of he iron bridge, is 65.50 feet above datum of gage. The west bank of the river is a solid wall of limestone, the east bank is flat and is subject to overflow. The trestle at the east end of the bridge is seven-eighths of a mile long. The section is good, though the water is very deep and rather swift. 
Daily gage height in feet of Tombigbee River near Epes, Ala., for 1900.

\begin{tabular}{|c|c|c|c|c|c|c|c|c|c|c|c|c|}
\hline Day & Jan, & Feb. & Mar. & April & May & Jun. & July & Aug. & Sept. & Oct. & Nov. & Des. \\
\hline & & उ.0 & 18.0 & 24.5 & 43.0 & 6.0 & & 14.5 & & 0.5 & 7.0 & \\
\hline & & & 19.0 & & 41.0 & 8.0 & 44.5 & 12.0 & 2.5 & .5 & 8.0 & \\
\hline & & 3.0 & 18.0 & & . 0 & 15.0 & 44.5 & 10.5 & 2.0 & .5 & 9.0 & 7.0 \\
\hline & 6.0 & 3.2 & 17.0 & 12.0 & 33.5 & 21.0 & 44.0 & 8.0 & 2.0 & .5 & 8.5 & 6.0 \\
\hline & & 5.0 & 15.0 & 10.0 & 28.0 & 24.5 & 42.5 & 6.0 & 2.0 & .5 & 8.5 & 5.0 \\
\hline & & 6.0 & $\begin{array}{l}14.0 \\
1.0\end{array}$ & 8.0 & 20.5 & 2.0 & ${ }^{42.0}$ & 5.0 & 2.0 & .5 & 7.5 & 4.5 \\
\hline & 4.0 & 7.5 & $\begin{array}{l}10.0 \\
18.0\end{array}$ & 7.5 & 10.0 & 32.0 & 40.0 & 4.0 & 2.0 & 5 & 5.5 & $\begin{array}{l}4.0 \\
4.0\end{array}$ \\
\hline & & 11.5 & 21.0 & 7.0 & 7.0 & 34.5 & 39.0 & 3.0 & 2.0 & .5 & 4.5 & $\begin{array}{l}4.0 \\
4.0\end{array}$ \\
\hline & 3.5 & 13.0 & 23.0 & 6.5 & 7.0 & 37.0 & 38.0 & 2.0 & 2.0 & 2.0 & & $\begin{array}{l}.00 \\
3.5\end{array}$ \\
\hline & 13.0 & 15.0 & 24.0 & 20.5 & 7.0 & 38.5 & 34.0 & 2.0 & 1.5 & 4.0 & & 3.0 \\
\hline & 0.0 & 20.5 & 24.0 & 0 & 8.0 & 39.5 & 26.0 & 20 & 1.5 & 6.0 & 3. & 3. \\
\hline & & 26.0 & 23.0 & 9.0 & 8.0 & 40.5 & & & 1. & 7.5 & & 3. \\
\hline & 23 & 28.0 & 20.0 & & 7.0 & 41.0 & & & 2.0 & 8.5 & & 3.5 \\
\hline & 22.0 & 28.0 & 17.5 & 31.0 & 6.0 & 41.5 & 13.0 & & 1.5 & 10.0 & & 3. \\
\hline & .0 & 26.0 & 17.0 & & 6.0 & 42.0 & 8.0 & & 0 & 0.5 & 2.0 & 3. \\
\hline & & 24 & 18.0 & & 5.0 & 42.0 & 7. & & & 10.0 & & 3.0 \\
\hline & & .0 & 18.0 & & $4: 0$ & 42.0 & 6. & & 1.0 & & & 3. \\
\hline & 11.5 & 18.5 & 18.5 & & 3.5 & 41.5 & 5. & & 1.0 & 5.0 & & 3.0 \\
\hline & 10.0 & 16.0 & 26.0 & & 3.5 & 41.5 & 6.5 & & .5 & 4.0 & 2.5 & 6.0 \\
\hline & 8. & $14 . v$ & 30.0 & & 3.5 & 41.5 & 7.0 & 1. & .5 & 3.5 & 3.0 & 5. \\
\hline & & & & & 3.5 & 41.0 & 8. & & .5 & 3.0 & & 5 \\
\hline & 3.8 & .5 & 34.0 & & 3.5 & & 9. & & & & & 8.5 \\
\hline & 7. & 18.0 & 35.5 & & 4.0 & 42.5 & 8. & & .5 & 2.0 & 3.5 & 10.0 \\
\hline & & & 37.5 & & 4.5 & & 6. & & .5 & 3.0 & 6. & 11.5 \\
\hline & 6. & .0 & & & 5.0 & 43.5 & 5. & & .5 & 4.0 & 8.5 & 11.5 \\
\hline & 50 & & & & 0.0 & 43.5 & & & .5 & 4.5 & 7.5 & 12.5 \\
\hline & 3.0 & 17.0 & 38.5 & 46.5 & 7.0 & 43.5 & 5.5 & 4.5 & & 7.5 & 7.5 & 12.0 \\
\hline & & $\cdots$ & $\begin{array}{l}\begin{array}{l}5.0 \\
33.0\end{array} \\
0\end{array}$ & $\begin{array}{l}46.0 \\
44.5\end{array}$ & $\begin{array}{l}7.0 \\
6.0\end{array}$ & $\begin{array}{l}44.1 \\
44.5\end{array}$ & $\begin{array}{l}14.0 \\
14.5\end{array}$ & $\begin{array}{l}4.0 \\
3.0\end{array}$ & $\cdot 5$ & 8.0 & 8.0 & 10.0 \\
\hline & 3. & & 30.0 & & 5.0 & & 14.5 & & & 7.5 & .... & 10.5 \\
\hline
\end{tabular}

The following discharge measurements were made during I90I by K. T. Thomas :

Jan. 31-Gage height, 12.70 feet; discharge, 13,738 second-feet. March 14-Gage height, 21.10 feet; discharge, 23,824 second-feet. June 28-Gage height, 1.00 feet; discharge, 1,496 second-feet.

Nov. 13-Gage height, 0.70 feet; discharge, 1,290 second-feet. 
Daily gage height in feet of Tombigbse River near Epes, Ala., for 1901.

\begin{tabular}{|c|c|c|c|c|c|c|c|c|c|c|c|c|}
\hline Day & Jan, & Feb. & Mar. & April & May & Jun. & July & Aug. & Sept. & Oct. & Nov. & Dec. \\
\hline & & 2.0 & 6.5 & 3.0 & 7.5 & 5.5 & 1.0 & & 5.5 & 1.5 & $0.7 \mid$ & \\
\hline & & & & & 7.0 & 6.0 & 1.0 & & 4.0 & 6.0 & 0.7 & \\
\hline & & 16.8 & 9.0 & 15.5 & 6.0 & 7.0 & 1.0 & & 3.5 & 2.5 & 0.7 & \\
\hline & & $\begin{array}{l}21.5 \\
25.5\end{array}$ & $\begin{array}{l}9.0 \\
8.5\end{array}$ & $\begin{array}{l}18.0 \\
17.0\end{array}$ & $\begin{array}{l}0.0 \\
4.5\end{array}$ & 9.0 & 1.0 & & 2.0 & $\begin{array}{l}1.5 \\
1.5\end{array}$ & $\begin{array}{l}0.0 \\
0.0\end{array}$ & 3. \\
\hline & 6.0 & 26.5 & 7.0 & 16.0 & 5.0 & 11.0 & 1.0 & & 2.0 & 1.5 & 0.0 & 2. \\
\hline & & 27,5 & 6.5 & 15.0 & 4.0 & 12.0 & 1.0 & & 1.5 & 1.5 & 0.0 & \\
\hline & & 29.0 & 6.5 & 14.0 & 3.5 & 10.0 & 1.0 & & 1.5 & 1.0 & 0.0 & \\
\hline & & 30.5 & 6.5 & 11.0 & 3.5 & 8.0 & 1.0 & & .5 & 1.0 & 0.7 & \\
\hline & & & .0 & 1.5 & 3.0 & 6.5 & 1.0 & & .5 & .0 & 0.7 & \\
\hline & & & & & 3.0 & 5.5 & 0.5 & & & 0 & 0.7 & \\
\hline & & & & 7.5 & 3.5 & 4.5 & 0.5 & & & & & \\
\hline & & & & & 4.0 & 4.0 & 0.5 & & & 0 & 0.7 & \\
\hline & & & & 8.0 & 7.0 & 3.5 & 0.5 & & & & 0.7 & 13 \\
\hline & & & 25. & 7.0 & 8.0 & 3.5 & 0.5 & & & & 0.7 & \\
\hline & & 2.0 & 26. & 7.0 & 8.5 & 3.5 & 0.5 & 8.5 & & & & \\
\hline & & & 26.5 & 6.5 & 8.0 & 3.0 & 0.5 & & & & 1.0 & \\
\hline & & 16.0 & & . 0 & 7.0 & 3.0 & 0.5 & & 2. & & 1.8 & \\
\hline & & & & & 6.5 & 2.5 & 0.5 & 22.5 & 6.0 & & & \\
\hline & & 1.00 & 23. & .0 & 9.0 & $2 . ?$ & $1.0^{\prime}$ & 23. & & & 1.5 & \\
\hline & & & 20.0 & & 12.0 & 2.0 & 1.0 & & 7.5 & & & \\
\hline & & & 17. & & 12.0 & 2. & 1.0 & & & & & 14 \\
\hline & & 7.5 & 12. & 3.5 & 11.5 & 1.5 & 1.0 & 26. & & & & 12 \\
\hline & & 7.0 & 11. & 28.0 & 11.0 & & 1.0 & & & & & \\
\hline & & 6.5 & $1<$. & 27.0 & 10.0 & 1.0 & & & & & & \\
\hline & & & 13. & & 8.0 & 1.0 & 1.0 & 25. & & & & \\
\hline & & & 13. & & & 1.0 & 0.5 & 23. & & 0.5 & 2.0 & 7. \\
\hline & & 6.5 & 11.5 & 13.0 & 6.5 & 1.0 & 0.5 & 19.0 & 1. & 0.5 & 2.0 & 12. \\
\hline & 14.0 & & 10.0 & 11.0 & 6.5 & 1.0 & & 12.0 & 1.5 & & 1.9 & en \\
\hline & $\begin{array}{l}13.0 \\
12.7\end{array}$ & & 3. & & 6.5 & & $?$ & & 1.5 & & 1.8 & \\
\hline & & & & & & & .2 & & & 0.5 & 0 & 27. \\
\hline
\end{tabular}


Rating table for Tombigbee River at Epes, Ala., for 1900-1901..

\begin{tabular}{|c|c|c|c|c|c|c|c|}
\hline 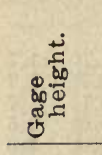 & 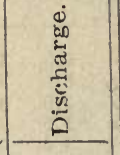 & 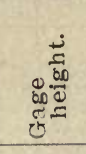 & $\begin{array}{l}\dot{0} \\
0 \\
0 \\
\tilde{0} \\
0 \\
0 \\
0 \\
0 \\
0 \\
\end{array}$ & 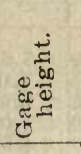 & 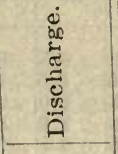 & 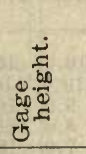 & 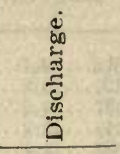 \\
\hline Feet. & second-ft. & Feet. & Second- $t$. & Feet. & Secon $-y t$. & Feet. & Second $f l$. \\
\hline-0.2 & 810 & 5.4 & 5,208 & 11.0 & 11,700 & 16.6 & 18,420 \\
\hline-0.1 & 840 & 5.5 & 5,308 & 11.1 & 11,820 & 16.7 & 18,540 \\
\hline 0.0 & 880 & 5.6 & 5,409 & 11.2 & 11,940 & 16.8 & 18,660 \\
\hline 0.1 & 830 & 5.7 & 5,511 & 11.3 & 12,060 & 16.9 & 18,780 \\
\hline 0.2 & 985 & 5.8 & 5,613 & 11.4 & 12,180 & 17.0 & 18,900 \\
\hline 0.3 & 1,043 & 5.9 & 5,716 & 11.5 & 12,300 & 17.1 & 19,620 \\
\hline 0.4 & 1,103 & 6.0 & 5,820 & 11.6 & 12,420 & 17.2 & 19,140 \\
\hline 0.5 & 1,164 & 6.1 & 5,925 & 11.7 & 12,540 & 17.3 & 19,260 \\
\hline 0.6 & 1,226 & 6.2 & 6,030 & 11.8 & 12,660 & 17.4 & 19,380 \\
\hline 0.7 & 1,289 & 6.3 & 6,136 & 11.9 & 12,780 & 17.5 & 19,500 \\
\hline 0.8 & 1,353 & 6.4 & 6,243 & 12.0 & 12,900 & 17.6 & 19,620 \\
\hline 0.9 & 1,418 & 6.5 & 6,350 & 12.1 & $\perp 3,020$ & 17.7 & 19,740 \\
\hline 1.0 & 1,484 & 6.6 & 6,458 & 12.2 & 13,140 & 17.8 & 19,860 \\
\hline 1.1 & 1,551 & 6.7 & $u, 566$ & 12.3 & 13,260 & 17.9 & 19,980 \\
\hline 1.2 & 1,619 & 6.8 & 6,675 & 12.4 & 13,380 & 18.0 & 20,100 \\
\hline 1.3 & 1,688 & 6.9 & 6,785 & 12.5 & 13,500 & 18.1 & 20,220 \\
\hline 1.4 & 1,758 & 7.0 & 6,900 & 12.6 & 13,620 & 18.2 & 20,340 \\
\hline 1.5 & 1,829 & 7.1 & 7,020 & 12.7 & 13,740 & 18.3 & 20,460 \\
\hline 1.6 & 1,903 & 7.2 & 7,140 & 12.8 & 13,860 & 18.4 & 20,580 \\
\hline 1.7 & 1,976 & 7.3 & 7,260 & 12.9 & 13,980 & 18.5 & 20,700 \\
\hline 1.8 & 2,050 & 7.4 & 7,380 & 13.0 & 14,100 & 18.6 & 20,020 \\
\hline 1.9 & 2,125 & 7.5 & 7,500 & 13.1 & 14,220 & 18.7 & 20,940 \\
\hline 2.0 & 2,200 & 7.6 & 7,620 & 13.2 & 14,340 & 18.8 & 21,060 \\
\hline 2.1 & 2,276 & 7.7 & 7,740 & 13.3 & 14,460 & 18.9 & 21,180 \\
\hline 2.2 & 2,353 & 7.8 & 7,860 & 13.4 & 14,580 & 19.0 & 21,500 \\
\hline 2.3 & 2,431 & 7.9 & 7,980 & 13.5 & 14,700 & 19.1 & 21,420 \\
\hline .2 .4 & 2,510 & 8.0 & 8,100 & 13.6 & 14,820 & 19.2 & 21,540 \\
\hline 2.5 & 2,590 & 8.1 & 8,220 & 13.7 & 14,940 & 19.3 & 21,660 \\
\hline 2.6 & 2,671 & 8.2 & 8,340 & 13.8 & 15,060 & 19.4 & 21,780 \\
\hline 2.7 & 2,753 & 8.3 & 8,460 & 13.9 & 15,180 & 19.5 & 21,900 \\
\hline 2.8 & 2,835 & 8.4 & 8,580 & 14.0 & 15,300 & 19.6 & 22,020 \\
\hline 2.9 & 2,918 & 8.5 & 8,700 & 14.1 & 15,420 & 19.7 & 22,140 \\
\hline 3.0 & 3,002 & 8.6 & 8,820 & 14.2 & 15,540 & 19.8 & 22,260 \\
\hline 3.1 & 3,087 & 8.7 & 8,940 & 14.3 & 15,660 & 19.9 & 22,380 \\
\hline 3.2 & 3,172 & 8.8 & 9,060 & 14.4 & 15,780 & 20.0 & 22,500 \\
\hline 3.3 & 3,258 & 8.9 & 9,180 & 14.5 & 15,900 & 20.1 & 22,620 \\
\hline 3.4 & 3,345 & 9.0 & 9,300 & 14.6 & 16,020 & 20.2 & 22,740 \\
\hline 3.5 & 3,432 & 9.1 & 9,420 & 14.7 & 16,140 & 20.3 & 22,860 \\
\hline 3.6 & 3,520 & 9.2 & 9,540 & 14.8 & $\cdot 16,260$ & 20.4 & 22,980 \\
\hline 3.7 & 3,609 & 9.3 & 9,660 & 14.9 & 16,380 & 20.5 & 23,100 \\
\hline 3.8 & 3,698 & 9.4 & 9,780 & 15.0 & 16,500 & 20.6 & 23,220 \\
\hline 3.9 & 3,788 & 9.5 & 9,900 & 15.1 & 16,620 & 20.7 & 23,340 \\
\hline 4.0 & 3,878 & 9.6 & 10,020 & 15.2 & 16,740 & 20.8 & 23,460 \\
\hline 4.1 & 3,969 & 9.7 & 10,140 & 15.3 & 16,860 & 20.9 & 23,580 \\
\hline 4.2 & 4,060 & 9.8 & 10,260 & 15.4 & 16,980 & 21.0 & 23,700 \\
\hline
\end{tabular}




\begin{tabular}{|c|c|c|c|c|c|c|c|}
\hline 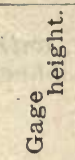 & 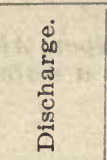 & 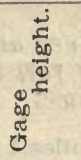 & 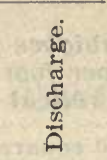 & 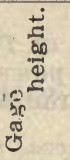 & 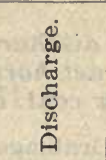 & & 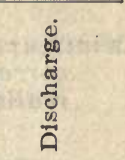 \\
\hline Feet. & Secnnd-ft. & Feet. & Second-ft. & F'eet & Second-ft. & Feet. & Second-feet. \\
\hline 4.3 & 4,152 & 9.9 & 10,380 & 15.5 & 17,100 & 21.1 & 23,820 \\
\hline 4.4 & 4,245 & 10.0 & 10,500 & 15.6 & 17,220 & 21.2 & 23,940 \\
\hline 4.5 & 4,338 & 10.1 & 10,620 & 15.7 & 17,340 & 21.3 & 24,06 () \\
\hline 4.6 & 4,432 & 10.2 & 10,740 & 15.8 & 17,460 & 21.4 & 24,180 \\
\hline 4.7 & 4,527 & 10.3 & 10,860 & 15.9 & 17,580 & 21.5 & 24,300 \\
\hline 4.8 & 4,622 & 10.4 & 10,980 & 16.0 & 17,700 & 21.6 & 24,420 \\
\hline 4.9 & 4,718 & 10.5 & 11,100 & 16.1 & 17,820 & 21.7 & 24,540 \\
\hline 5.0 & 4,815 & 10.6 & 11,220 & 16.2 & 17,940 & 21.8 & 24,660 \\
\hline 5.1 & 4,912 & 10.7 & 11,340 & 16.3 & 18,060 & 21.9 & 24,780 \\
\hline 5.2 & 5,010 & 10.8 & 11,460 & 16.4 & 18,180 & 22.0 & 24,900 \\
\hline 5.3 & 5,109 & 10.9 & 11,580 & 16.5 & 18,300 & & \\
\hline
\end{tabular}

Note.-This table applied to the foregoing "Daily gage heights" gives the cubic feet per second flowing in the river on each date for which the gage height is given.

Estimated monthly discharge of Tombigbee River at Epes, Ala.

[Drainage area, 8,830 square miles.]

\begin{tabular}{|c|c|c|c|c|c|}
\hline \multirow[b]{2}{*}{ Month. } & \multicolumn{3}{|c|}{ Discharge in second-feet. } & \multicolumn{2}{|c|}{ Run-off. } \\
\hline & $\begin{array}{l}\text { Maxi- } \\
\text { mum. }\end{array}$ & $\begin{array}{l}\text { Mini- } \\
\text { mum. }\end{array}$ & Mean. & $\begin{array}{c}\text { Second. } \\
\text { feet per } \\
\text { square } \\
\text { mile. }\end{array}$ & $\begin{array}{l}\text { Depth } \\
\text { in } \\
\text { inches. }\end{array}$ \\
\hline 1901. & & & & & \\
\hline January . & 47,100 & 3,878 & 25,579 & 2.90 & 3.34 \\
\hline February . & 35,700 & 6,350 & 20,999 & 2.38 & 2.48 \\
\hline March ........ & 30,300 & 6,350 & 16,198 & 1.83 & 2.11 \\
\hline April $\ldots \ldots \ldots$ & 34,500 & 6,350 & 18,102 & 2.05 & 2.29 \\
\hline May $\ldots . . . \ldots \ldots$ & 12,900 & 3,002 & 6,880 & .78 & .90 \\
\hline June $\ldots . . . \ldots \ldots \ldots$ & 12,900 & 1,484 & 4,585 & .52 & .58 \\
\hline July $\ldots \ldots \ldots \ldots \ldots \ldots$ & 1,484 & 810 & 1,295 & .15 & .17 \\
\hline August $16-31 \ldots \ldots \ldots \ldots \ldots$ & & $\ldots \ldots \ldots$ & 21,541 & 2.44 & 1.41 \\
\hline September $\ldots \ldots \ldots \ldots \ldots \ldots$ & 7,500 & 1,484 & 3,205 & .36 & .40 \\
\hline October $\ldots \ldots \ldots \ldots \ldots$. & 5,820 & 1,164 & 1,633 & .18 & .21 \\
\hline November ............... & 2,200 & 880 & 1,550 & .18 & .20 \\
\hline December $\ldots \ldots \ldots \ldots \ldots \ldots$ & 30,900 & 1,960 & 12,249 & 1.39 & 1.60 \\
\hline
\end{tabular}


Minimum monthly discharge of Tombigbee River at Epes, Ala., with corresponding net horse power per foot of fall on a water wheel realizing 80 per cent. of the theoretical power.

[Drainage area, 8,830 square miles.]

\begin{tabular}{|c|c|c|c|c|c|c|}
\hline \multirow[b]{2}{*}{ 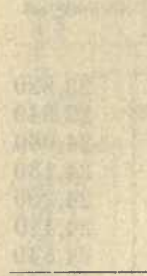 } & \multicolumn{3}{|c|}{1900.} & \multicolumn{3}{|c|}{$19 i 1}$. \\
\hline & 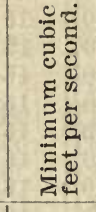 & 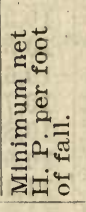 & 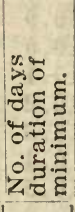 & 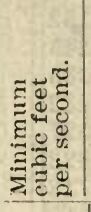 & 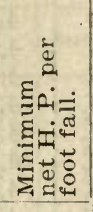 & 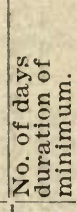 \\
\hline January & 3,002 & 273 & 4) & 3,878 & 353 & 1 \\
\hline February & 3,002 & 273 & 4 & 6,350 & 577 & 4 \\
\hline March ... & 14,100 & 1,282 & 1) & 6,350 & 577 & 5 \\
\hline April ... & 6,350 & 577 & 1 & 6,350 & 577 & 1 \\
\hline May .... & 3,432 & 312 & 5 & 3,002 & 273 & 2 \\
\hline June .... & 5,820 & 529 & 1 & 1,484 & 135 & 6 \\
\hline July .... & 4,815 & 438 & 2 & 810 & 74 & 3 \\
\hline August .. & 1,484 & 135 & 2 & & & \\
\hline September & 1,164 & 106 & 11 & 1,484 & 135 & 3 \\
\hline October. & 1,164 & 106 & 9 & 1,164 & 106 & 8 \\
\hline November & 2,200 & 200 & 4 & 880 & 80 & 5 \\
\hline December & 3,002 & 273 & 5 & 1,960 & 178 & 3 \\
\hline
\end{tabular}

Note-To find the minimum net horse power available at a shoal on this stream, near this station, for any month, multiply the total fall of the shoal by the "net H. P. per foot of fall" in this table for that month.

\section{TRIBUTARIES.}

There are several large creeks in Marion and Lamar Counties that flow into Mississippi, and enter the Tombigbee River near Columbus. One of these, the Buttahatchee Creek, in Marion County, has numerous rapids, especially near the crossing of the Military Road.

Luxapalila Creek, in Lamar County, has two prongs that are both good power streams. They come together before the creek enters Mississippi, making Big Luxapalila Creek, which enters the Tombigbee at Columbus, Miss. The following measurements have been made on this streani at Columbus, Miss.:

1901.

March 11-Gage height, 8.20 feet; discharge, 2,459 second-feet.

April 16-Gage height, 4.45 feet; discharge, 873 second-feet.

June 26-Gage height, 1.90 feet; discharge, 109 second-feet.

Oct. 31 -Gage height, 2.00 feet; discharge, 126 second-feet. 


\section{CHAPTER VII.}

1. TENNESSEE RIVER AT CHATtANOOGA, TENNESSEE.

This river, after passing Chattanooga, enters Alabama. It then makes a bend to the west and later to the north, returning to Tennessee. Flowing through this State and Kentucky, it empties into the Ohio 50 miles above Cairo. In 1879 a gage was established at Chattanooga, Tennessee, at the foot of Lookout street, just below Chattanooga Island, by the Signal Corps of the United States Army, which has been in charge of the Weather Bureau since July I, I89r. The drainage area above this station is 21,382 square miles, and is mapped on Morristown, Greenville, Roan Mountain, London, Knoxville, Mount Guyot, Asheville, Murphy, Briceville, Standingstone, Wartburg, Pikeville, Maynardville, Cumberland Gap, Jonesville, Estillville, Bristol, Whitesburg, Grundy, Abington, Tazewell, Pocahontas, Wytheville, Cranberry, Morganton, Mount Mitchell, Saluda, Pisgah, Como, Nantahala, Walhalla, Dahlonega, Ellijay, Dalton, Cleveand, Ringgold, Kingston, and Chattanooga atlas sheets. The gage is on an incline railroad iron for about 20 feet of its lower portion. Above this it is a vertical rorl, bolted to the rock bluff forming the river bank. The zero of the gage is 630.4 feet above sea level. Measurements are made from the Hamilton County steel highway bridge at the foot of Walnut street, a short distance below the gage. Gage heights are obtained from L. M. Pindell, United States Weather Bureau observer. During the year I900 a new gage on the same datum was established. It is a vertical rod bolted to the south side of the third stone pier from the south end of the bridge. 
Daily gage height of Tennessee River at Chattanooga, Tenn, for 1890.

[Furnished by L. M. Pindell, observer in charge, United States Weather Bureau.]

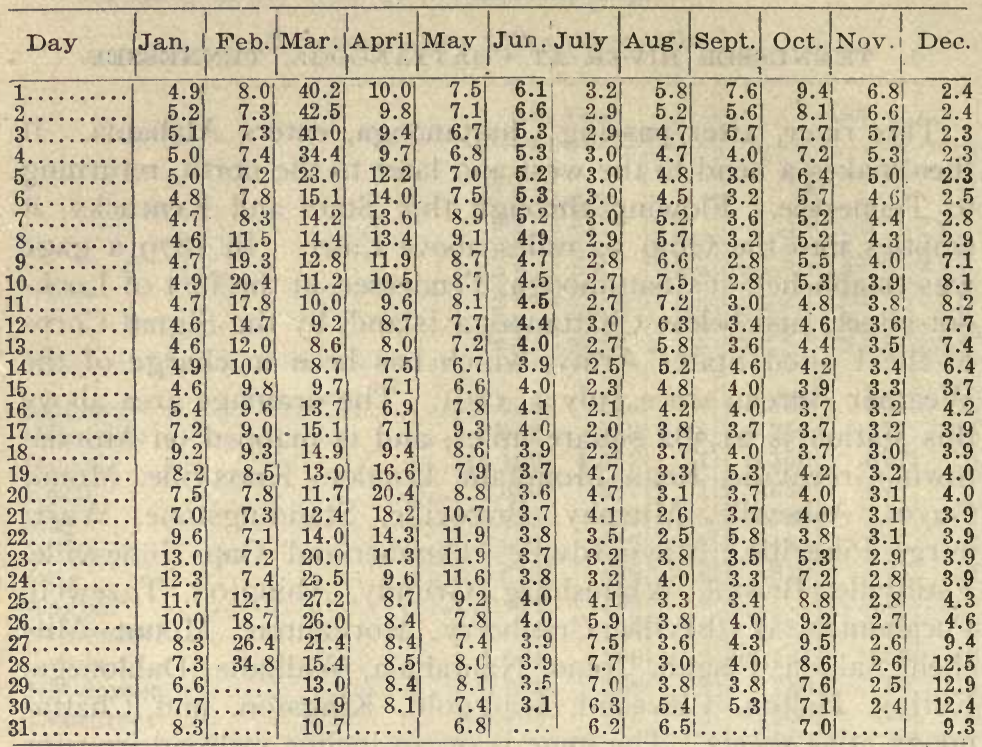

Daily gage height of Tennessee River at Chattanooga, Tenn, for 1891.

\begin{tabular}{|c|c|c|c|c|c|c|c|c|c|c|c|c|}
\hline Day & n, & & Mar. & April & May & Jun. & July & Aug. & Sept. & Oct. & Nov. & Dec \\
\hline & 7.7 & 9.8 & 18.6 & 15.4 & 5.9 & $\overline{5.8}$ & 3.9 & 8.3 & 5.4 & 1.8 & $\overline{1.3}$ & 3.7 \\
\hline & 7.8 & 13.2 & 17.5 & & & & & 10.3 & & & & \\
\hline & 9.9 & 16.1 & 15.6 & 16 & 6 & 5.3 & 3.6 & 15.1 & 4.3 & 1.9 & 1.3 & \\
\hline & 14.1 & 19.8 & 13.3 & 15.7 & 5.5 & 4.8 & 3.6 & 16.4 & 4.2 & 1.8 & 1.2 & 2. \\
\hline & & 22.6 & 15.4 & 15.1 & 5.2 & 4.4 & 3.6 & 12.0 & 5.1 & 1.8 & 1.2 & 5. \\
\hline & 15.2 & 21.6 & 20.0 & 12.6 & 5.1 & 4.1 & 3.6 & 8.7 & 5.1 & 1.7 & 1.2 & 6. \\
\hline & 10.4 & 18.3 & 23.6 & 11.6 & 4.9 & 3.9 & 3.4 & 6.9 & 5.2 & 1.7 & & 6. \\
\hline & 8.2 & 16.9 & 29.1 & 10.8 & 4.7 & 4.1 & 3.4 & 5.8 & 5.2 & 1.6 & 1.2 & 8. \\
\hline & $\begin{array}{l}0.1 \\
7.1\end{array}$ & $\begin{array}{l}10.9 \\
14.5\end{array}$ & 34.5 & 9.8 & 4.6 & 4.6 & $\begin{array}{l}0.4 \\
3.3 \\
3.3\end{array}$ & 5.1 & 5. & 1.7 & & 10. \\
\hline & 6.3 & 21.0 & $\begin{array}{l}57.0 \\
37.5\end{array}$ & 9.6 & 4.5 & 4.7 & $\begin{array}{l}0.0 \\
4.5\end{array}$ & 4.6 & 4. & 1.7 & & 10. \\
\hline & & 27.8 & 38.9 & 9.8 & 4.4 & 5.6 & 5. & 4. & & 1.8 & & 10 . \\
\hline & 8.9 & 34.3 & 37.6 & 9.9 & 4.3 & 7. & & 4. & & 1.8 & & 8. \\
\hline & 10.7 & 36.5 & 33.5 & 10.6 & 4.2 & 6. & & 4. & & & & \\
\hline & 10.0 & & 27.0 & 11.3 & & & & 3.9 & & 1.7 & & 5. \\
\hline & & & 22.2 & 12.2 & 4.2 & 5. & & 3.8 & & & & \\
\hline & 7.3 & 29 . & 19.8 & 10.8 & & 5. & & 3.6 & & & & \\
\hline & 7.8 & 21.1 & 18.1 & & & 5. & & 3.5 & & & & \\
\hline & 7.5 & 19. & 15.3 & 8.4 & 4.7 & 6 & & 3.4 & & & & 5 \\
\hline & 7.5 & & & 8.2 & & & & 3. & & & & \\
\hline & 7.6 & & 12. & 7.9 & & 7. & & & & & & \\
\hline & & & 11. & 7.9 & & 6. & & & & & & \\
\hline & 8.2 & & 10. & 7.6 & 4 & 6. & & 4.0 & & & & \\
\hline & 12.5 & & 10. & 7.4 & 4.1 & 6. & & 4. & & & & \\
\hline & & & 10 & 7.4 & & 7. & & & & & & 3. \\
\hline & 14.0 & 29. & 10 . & 7.5 & 3. & 7. & 3. & 5.6 & & & 6.2 & 4. \\
\hline & 13.6 & & 10. & 7.5 & & 7. & & & & & 6. & 4. \\
\hline & 11.2 & 20.6 & 10. & 7.4 & $4 . n$ & & & 8.2 & & & & 8. \\
\hline & 9.71 & 19.0 & 14.1 & 7.2 & 4. & 4. & 3. & 8. & & 1. & 5.6 & 10. \\
\hline & 7.9 & & 13. & 6.5 & 4. & 4.3 & & 7.0 & 1. & & $4 . ?$ & 9. \\
\hline & 7.9 & .. & 13.0 & 6.2 & 4.7 & 4.1 & 3.8 & 6.4 & 1.9 & 1.4 & 4.0 & 8. \\
\hline & 8.9 & & 13.1 & & 5.3 & & 5.7 & 6.1 & .... & 1.4 & ....... & 7.8 \\
\hline
\end{tabular}




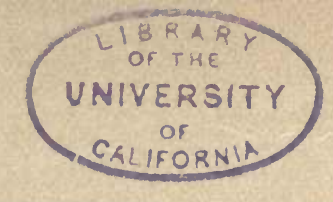

WATER-POWERS OF ALABAMA.

141

Daily gage lheight of Tennessee River at Chattanooga, Tenn, for 1892.

\begin{tabular}{|c|c|c|c|c|c|c|c|c|c|c|c|c|}
\hline Day & Jan, & Feb. & Mar. & April & May & Jun. & July & Aug. & Sept. & Oct. & Nov. & Dec \\
\hline & & & & & & & & 4.2 & & & & \\
\hline & & & & & 8.2 & & & 4.1 & 1.8 & 2.0 & & \\
\hline & & $\begin{array}{l}6.0 \\
5.8\end{array}$ & $\begin{array}{l}5.5 \\
5.3\end{array}$ & .4 & $\begin{array}{l}7.6 \\
7.3\end{array}$ & $\begin{array}{l}5.5 \\
5.8\end{array}$ & $\begin{array}{l}5.5 \\
5.4\end{array}$ & $\begin{array}{l}4.2 \\
4.0\end{array}$ & $\begin{array}{l}1.7 \\
1.5\end{array}$ & $\begin{array}{l}1.9 \\
1.8\end{array}$ & $\begin{array}{l}1.2 \\
1.6\end{array}$ & 8 \\
\hline & & 5.5 & 5.1 & & 7.4 & 8.8 & & 4.1 & 1.3 & $\begin{array}{l}1.7 \\
\end{array}$ & 2.0 & \\
\hline & & 5.4 & 5.0 & & 7.0 & 9. & 8.9 & 4.9 & 2.2 & 1.6 & 2.3 & \\
\hline & & & 4.9 & 21.7 & 6.6 & 8. & 11.2 & 4.2 & 2.1 & 1.6 & 2.4 & \\
\hline & 100 & 8.8 & 6.0 & 3 & 6.5 & 8.1 & 1.8 & & & & 2.4 & \\
\hline & 9.3 & 11.5 & 7.1 & 34. & & 8.3 & & & & & & \\
\hline & & 11.3 & 8.0 & & 5. & & & & & & & \\
\hline & & 10.5 & 7.9 & & & & & & & & & \\
\hline & & 8.9 & 7.6 & & & & & & & & & \\
\hline & & 7.7 & 7.6 & & & & & 3.6 & 2. & 1.4 & 4.4 & \\
\hline & $\begin{array}{r}32.9 \\
27\end{array}$ & 7.2 & 6.8 & 11.7 & 5.3 & & 8. & 3.7 & 2.1 & 1.4 & 4.2 & \\
\hline & 37.1 & 7.4 & 6.2 & 10.9 & 5.2 & 5. & & 3. & & & & \\
\hline & & 70 & . & 10. & b.1 & 4. & & 3. & & & & 5 \\
\hline & & 65 & 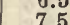 & & & 4. & & & & & 6.2 & \\
\hline & & 1.0 & 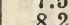 & & 0. & 4. & & & & & & \\
\hline & 19.0 & $\because 0$ & 8.4 & & & & & & & & & \\
\hline & $\begin{array}{l}10.0 \\
19.0\end{array}$ & 89 & 7.2 & & & 7. & & & & & & \\
\hline & & 8.9 & 7.6 & 16.3 & 6.5 & 7. & & 2. & & 1. & & 9 \\
\hline & & 8.4 & & & & & & & & & & \\
\hline & & 7.9 & & & & & & & & & & \\
\hline & 10.5 & 7.5 & 9.5 & & & & & & & & & 5 \\
\hline & 9. & 6.7 & & & & & & & & & & 5. \\
\hline & 8. & 6.4 & & & & & & & & 1.1 & & 4. \\
\hline & 7.7 & 5.9 & & & & & & & & .1 & & 4. \\
\hline & & $\cdots$ & & & 5. & 7.2 & & & 2.4 & 1 & 3.6 & 3. \\
\hline & & & & & & & & & & 1.1 & $\cdots$ & \\
\hline
\end{tabular}

List of discharge measurements madt on Tennessee River at Chattanooga, Tennessee.*

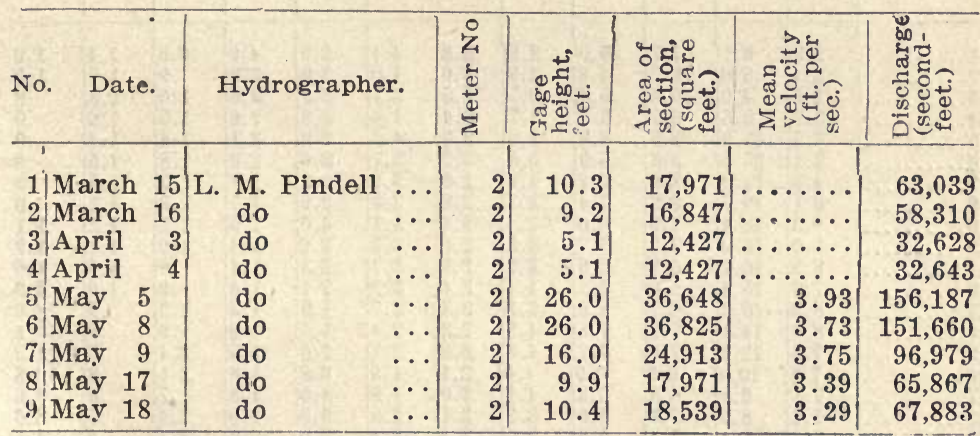

*Meter submerged at one-half depth. 
Daily gage height of Tennessee River at Chattanooga, Tenn., for 1893.

\begin{tabular}{|c|c|c|c|c|c|c|c|c|c|c|c|c|}
\hline Day & Jan, & Feb. & Mar. & April & May & Jun. & fuly & Aug. & ept. & Oct. & Nov. & De \\
\hline & & .4 & 8.4 & 5.5 & 10.2 & 7.4 & 3.9 & 2.6 & 8.4 & 2.6 & 3.1 & \\
\hline & & & & 5.3 & 9.6 & & & & & 2.5 & 3.0 & \\
\hline & & 0.6 & 8.9 & 5.1 & 11.0 & 10.0 & .2 & 3..1 & 5.0 & 2.4 & .8 & \\
\hline & & & 8. & 5.1 & 8.4 & & & 3.5 & 6. & & .7 & \\
\hline & & & 8.7 & 5.1 & 24.5 & & 1.8 & 3.3 & 6.0 & .6 & 2.6 & \\
\hline & & 7.7 & 9.0 & 5.2 & 8.2 & & & 3.2 & 4. & & 2.5 & \\
\hline & & 7.0 & 9.1 & 5.4 & 30.0 & 16.0 & 3.8 & 4.9 & 4.3 & 2.9 & 2.4 & \\
\hline & & 6.5 & 8.8 & 5.4 & 28.2 & & 3.5 & 5 & 3. & & 2.4 & \\
\hline & & 6.1 & 8.8 & 5.1 & 18.0 & 19.1 & 3.4 & 4.1 & 3.2 & 2. & 2.3 & \\
\hline 0. & & 6.2 & 9.4 & 5.1 & 12.8 & & .6 & 3.8 & 2. & & 2.4 & \\
\hline & & 8.5 & 11.1 & 5.1 & 11.7 & 11.8 & .4 & 3.3 & 2. & 2. & & \\
\hline & & 14.7 & 1.7 & 5.0 & 10.4 & & .4 & 3.0 & 3. & & & \\
\hline & 2. & & 11.5 & 4.8 & 9.4 & & 3.3 & 2.7 & 5. & & & \\
\hline & & 23.6 & 12.0 & 10.2 & 8.8 & & 3.2 & 2.5 & 10.9 & & 3.6 & \\
\hline & * & & 1 & 12.1 & 8.1 & & 3.0 & 2.8 & 12. & & & \\
\hline & * & 21.3 & 9 & 10.4 & 7.8 & 6 & 2.8 & 2 & & . & & \\
\hline & * & & 8. & 8.6 & 9.4 & 5 & & & & & 2. & \\
\hline & * & & 7.6 & 7.4 & 10.4 & 5 & 2.8 & 5. & 7. & & & \\
\hline & * & & 7. & 6.5 & 8.9 & & 3. & 4. & 6. & ( & $\overline{2}$. & \\
\hline & * & 33.4 & 6.7 & 6.4 & 7.7 & 5. & 3.2 & 4. & & & 2 & \\
\hline & * & & 6. & 7.2 & 7.4 & 5 & 3.5 & 2. & 4.2 & 1 & 2 & \\
\hline & * & 28.5 & 6. & 7.2 & 6.7 & & 3. & 2.6 & & & & \\
\hline & 2.9 & 18 & 5. & 7.1 & 6.1 & & 4. & 2. & & 3. & 2. & 3 \\
\hline & 3. & 12.3 & 5. & 6.8 & 5.7 & & 5 & 2 & & & 2. & \\
\hline & 3.1 & 10.4 & 6 & 6.7 & 5. & & 5. & 2. & & $\vdots$ & & \\
\hline & 3.4 & 9.3 & 6. & 6.0 & 5.2 & & 3. & 1 & & 3 & & \\
\hline & 3.7 & 8.4 & 6. & 5.7 & 5.0 & 5 & 3. & 1. & & 4. & & 2. \\
\hline & 3.8 & 8.2 & 6. & 7.0 & 4.6 & & 2. & 1. & 2.6 & 4. & 2 . & \\
\hline & 4.4 & & 5.9 & 9.5 & 5. & 4 & 3. & 1. & 2. & 4 & 2. & \\
\hline & 5.3 & & 5.8 & 10.4 & 6. & 4. & 2 & 1. & 2.5 & 3.5 & 2.5 & 2 \\
\hline $31, \ldots \ldots \ldots$ & 7.1 & & 5.7 & & & & 2.6 & 1.6 & & 3.2 & & 3. \\
\hline
\end{tabular}

*Frozen at gage.

Daily gage height of Tennessee River at Chattanooga, Tenn., for 1894 .

\begin{tabular}{|c|c|c|c|c|c|c|c|c|c|c|c|c|}
\hline Day & Jan, & Feb. & Mar. & Apri & May & Jun. & uly & Aug. & Sept. & Oct. & Nov. & Dec. \\
\hline $\begin{array}{l}21 \ldots \ldots \\
22 \ldots \ldots \\
23 \ldots \ldots \\
24 \ldots \ldots \\
25 \ldots \ldots \\
26 \ldots \ldots \\
27 \ldots \ldots \\
28 \ldots \ldots \\
39 \ldots \ldots \\
31 \ldots \ldots \\
\end{array}$ & $\begin{array}{l}2.9 \\
3.4 \\
3.8 \\
3.9 \\
3.5 \\
3.1 \\
4.9 \\
6.1 \\
9.3 \\
9.0 \\
8.5 \\
7.9 \\
8.3 \\
8.0 \\
7.8 \\
7.8 \\
7.1 \\
7.2 \\
6.3 \\
6.0 \\
5.3 \\
5.0 \\
5.0 \\
5.4 \\
5.3 \\
5.2 \\
5.4 \\
5.4 \\
5.1 \\
5.0 \\
4.9\end{array}$ & $\begin{array}{r}5.1 \\
5.0 \\
4.9 \\
5.5 \\
21.9 \\
2.5 \\
23.9 \\
19.7 \\
16.1 \\
16.0 \\
16.7 \\
15.4 \\
15.2 \\
14.1 \\
12.2 \\
10.5 \\
9.5 \\
8.6 \\
8.4 \\
8.3 \\
8.5 \\
8.7 \\
8.8 \\
8.2 \\
7.9 \\
7.0 \\
7.7 \\
7.7 \\
\ldots \ldots . \\
\ldots . .\end{array}$ & $\begin{array}{l}7.7 \\
8.2 \\
9.4 \\
9.7 \\
9.5 \\
9.3 \\
8.5 \\
8.2 \\
7.9 \\
7.2 \\
6.9 \\
6.6 \\
6.7 \\
7.2 \\
7.0 \\
6.9 \\
6.8 \\
7.3 \\
7.4 \\
7.7 \\
7.1 \\
8.8 \\
8.8 \\
8.1 \\
7.7 \\
7.3 \\
7.0 \\
6.5 \\
5.9 \\
5.7 \\
5.2\end{array}$ & $\begin{array}{l}5.0 \\
4.8 \\
5.4 \\
5.3 \\
6.8 \\
6.9 \\
7.2 \\
7.4 \\
6.6 \\
5.7 \\
5.9 \\
7.2 \\
8.5 \\
7.8 \\
7.2 \\
6.9 \\
6.3 \\
5.5 \\
5.0 \\
5.1 \\
4.9 \\
4.8 \\
4.7 \\
4.6 \\
4.5 \\
4.3 \\
4.2 \\
4.1 \\
4.0 \\
4.0\end{array}$ & $\begin{array}{l}3.9 \\
3.8 \\
3.7 \\
3.7 \\
3.6 \\
3.5 \\
3.4 \\
3.3 \\
3.4 \\
3.3 \\
3.2 \\
4.7 \\
5.1 \\
4.8 \\
4.3 \\
4.0 \\
4.1 \\
5.2 \\
5.0 \\
5.4 \\
5.6 \\
6.2 \\
6.8 \\
6.9 \\
7.1 \\
6.7 \\
6.0 \\
5.6 \\
5.1 \\
4.7 \\
4.2\end{array}$ & $\begin{array}{l}3.8 \\
3.6 \\
3.5 \\
3.4 \\
3.3 \\
3.2 \\
2.9 \\
2.8 \\
2.6 \\
2.5 \\
2.5 \\
2.4 \\
2.3 \\
2.3 \\
2.2 \\
2.1 \\
2.0 \\
2.1 \\
2.4 \\
2.5 \\
2.5 \\
2.5 \\
2.3 \\
2.2 \\
2.2 \\
2.5 \\
2.6 \\
2.7 \\
4.3\end{array}$ & $\begin{array}{l}4.4 \\
4.0 \\
3.7 \\
4.4 \\
4.2 \\
3.7 \\
3.2 \\
3.3 \\
3.1 \\
3.3 \\
3.7 \\
3.3 \\
2.7 \\
2.4 \\
.1 \\
1.9 \\
1.8 \\
2.8 \\
2.4 \\
2.4 \\
3.3 \\
3.7 \\
3.8 \\
3.4 \\
4.0 \\
1.4 \\
3.9 \\
3.8 \\
3.6 \\
3.3 \\
3.3\end{array}$ & $\begin{array}{l}2.9 \\
2.9 \\
2.9 \\
2.8 \\
2.9 \\
3.0 \\
2.9 \\
2.9 \\
3.0 \\
2.6 \\
2.3 \\
2.1 \\
1.9 \\
1.8 \\
2.0 \\
3.6 \\
4.6 \\
3.5 \\
3.0 \\
3.1 \\
3.6 \\
3.7 \\
4.0 \\
3.6 \\
3.0 \\
2.6 \\
2.2 \\
2.7 \\
2.4 \\
2.7\end{array}$ & $\begin{array}{r}4.0 \\
3.8 \\
3.0 \\
2.6 \\
2.1 \\
2.0 \\
1.8 \\
1.7 \\
1.5 \\
1.4 \\
1.4 \\
1.4 \\
1.3 \\
1.2 \\
1.5 \\
1.8 \\
2.0 \\
2.0 \\
1.6 \\
1.5 \\
1.5 \\
1.8 \\
1.8 \\
1.3 \\
1.1 \\
1.0 \\
.9 \\
.8\end{array}$ & $\begin{array}{r}0.9 \\
.9 \\
1.0 \\
1.5 \\
1.8 \\
1.5 \\
1.3 \\
1.1 \\
1.0 \\
.8 \\
.9 \\
1.2 \\
1.9 \\
2.1 \\
1.7 \\
1.4 \\
1.1 \\
1.0 \\
.9 \\
.8 \\
.8 \\
.8 \\
.8 \\
.7 \\
.7 \\
1.7 \\
1.1\end{array}$ & $\begin{array}{r}1.4 \\
1.7 \\
2.6 \\
1.4 \\
1.5 \\
1.5 \\
1.2 \\
1.1 \\
1.0 \\
.9 \\
.8 \\
.8 \\
.8 \\
.7 \\
.9 \\
.9 \\
.9 \\
1.0 \\
1.1 \\
1.2 \\
1.2 \\
1.1 \\
1.1 \\
1.2\end{array}$ & $\begin{array}{r}1.0 \\
1.0 \\
.9 \\
.9 \\
.9 \\
.9 \\
.9 \\
.9 \\
1.1 \\
1.2 \\
1.6 \\
38 \\
\times .6 \\
111 \\
11.2 \\
11.8 \\
.6 \\
6.6 \\
4.7 \\
4.2 \\
3.6 \\
3.2 \\
2.8 \\
2.7 \\
2.5 \\
2.4 \\
4.2 \\
6.9 \\
8.4 \\
7.9 \\
5.8\end{array}$ \\
\hline
\end{tabular}


Daily gage height of Tennessee River at Chattanooga, Tenn., for 1895 .

\begin{tabular}{|c|c|c|c|c|c|c|c|c|c|c|c|c|}
\hline Day & Jan, & Feb. & Mar. & April & May & Jun. & July & Aug. & Sept. & Oct. & Nov. & Dec. \\
\hline & & & 6.8 & 7.8 & & & & 4.4 & 3.3 & & & \\
\hline & & & 7.3 & & & 5.2 & 3.4 & & 3.4 & .8 & 1.2 & 1.5 \\
\hline & $\begin{array}{l}3 . \\
3 .\end{array}$ & $\begin{array}{l}7.3 \\
7.5\end{array}$ & $\begin{array}{l}12.1 \\
18.2\end{array}$ & $\begin{array}{l}6.8 \\
6.5 \\
\end{array}$ & $\begin{array}{l}5.5 \\
5.4\end{array}$ & $\begin{array}{l}4.8 \\
4.5\end{array}$ & $\begin{array}{l}3.8 \\
440\end{array}$ & & $\begin{array}{l}3.2 \\
3.1\end{array}$ & $\begin{array}{l}8 \\
8 \\
8\end{array}$ & 1.3 & 1.4 \\
\hline & & & & 6.3 & 5.7 & 4.2 & 4.0 & & 2. & 8 & 1.6 & 1 \\
\hline & & 7. & 18.2 & 6.2 & 6.0 & 4. & 5.0 & & 2. & .8 & & \\
\hline & & 6.9 & 13.4 & 6.0 & 6.5 & 4.6 & 5.0 & 3.2 & 2.8 & 8 & 1.3 & 1 \\
\hline & & 6.5 & 10.5 & 9.6 & & & 5.1 & & 2. & .9 & 1.2 & \\
\hline & & & 9.2 & 10.7 & & & 5.5 & & 2. & 8 & 1.1 & \\
\hline & & & 8 & & 8. & & & & & .9 & 1.2 & \\
\hline & & & 8.1 & & & & & & & 1.0 & 1.3 & \\
\hline & & & 7.5 & & & & & & & 1.0 & 1.7 & \\
\hline & & 4.2 & 7.8 & 10. & & & & & & 1.0 & & \\
\hline & & & 8.0 & & & & & & & 1.0 & 2.4 & \\
\hline & & 4.3 & 8.7 & & & & & & & .9 & & \\
\hline & & & 9.4 & 7.4 & & & & & & 1.0 & & \\
\hline & & & & & & & & & & & & \\
\hline & & & 9.6 & 9 & & & & & & 1.0 & & \\
\hline & & & 9. & 11 & & & & & & .9 & & \\
\hline & & & & & & & & & & .9 & & \\
\hline & & & & & & & & & & .8 & & \\
\hline & 10. & & & & & & & & & .8 & & \\
\hline & & & & & & & 2. & & & .7 & & \\
\hline & & 6.7 & & 7.1 & & 3. & 2. & & & .7 & & \\
\hline & & 6.8 & & & 5. & 2. & 2. & & & & 1. & \\
\hline & & 6. & & & & & & & & & & 4 \\
\hline & & 6. & & & & & & & & & & \\
\hline & 9. & 6.3 & 10. & & 7. & & 0 & & & & & \\
\hline & & & & & & & & & & & & \\
\hline & & & & .9 & & 2.6 & & & & .7 & 1.5 & \\
\hline & & & 8 & & 6.0 & & 5.3 & 3.4 & & 1.0 & .... & 4.2 \\
\hline
\end{tabular}

*Frozen. 
Rating table for Tennessee River at Chattanooga, Tennessee.

['This table is applicable from Jan, 1, 1890, to Dec. 31, 1895.]

\begin{tabular}{|c|c|c|c|c|c|c|c|}
\hline 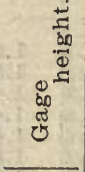 & 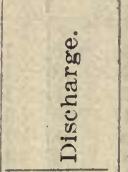 & & 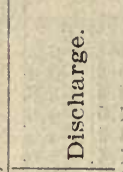 & 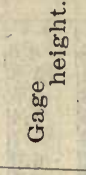 & 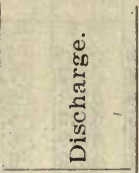 & 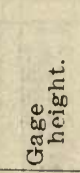 & 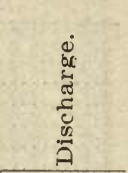 \\
\hline Feet. & Second $f t$. & Feet. & Second $f t$. & Feet. & Second ft. & Feet. & second $f t$. \\
\hline 0.7 & 16,360 & 11.0 & 66,850 & 22.5 & 133,665 & 34.0 & 293,820 \\
\hline 0.8 & 16,560 & 11.5 & 69,755 & 23.0 & 136,570 & 34.5 & 302,720 \\
\hline 0.9 & 16,780 & 12.0 & 72,660 & 23.5 & 139,475 & 35.0 & 311,620 \\
\hline 1.0 & 17,000 & 12.5 & 75,565 & 24.0 & 142,380 & 35.5 & 320,520 \\
\hline 1.5 & 18,160 & 13.0 & 78,470 & 24.5 & 145,285 & 36.0 & 329,420 \\
\hline 2.0 & 19,500 & 15.5 & 81,375 & 25.0 & 148,190 & 36.5 & 338,320 \\
\hline 2.5 & 21,100 & 14.0 & 84,280 & 25.5 & 151,095 & 37.0 & 347,220 \\
\hline 3.0 & 23,000 & 14.5 & 87,185 & 26.0 & 154,000 & 37.5 & 356,120 \\
\hline 3.5 & 25,090 & 15. & 90,090 & 26.5 & 162,500 & 38.0 & 365,020 \\
\hline 4.0 & 27,300 & 15.5 & 92,995 & 27.0 & 171,000 & 38.5 & 373,920 \\
\hline 4.5 & 29,660 & 16.0 & 95,900 & 27.5 & 179,900 & 39.0 & 382,820 \\
\hline 5.0 & 32,200 & 16.5 & 98,805 & 28.0 & 188,800 & 39.5 & 391,720 \\
\hline 5.5 & 34,895 & 17.0 & 101,710 & 28.5 & 197,700 & 40.0 & 400,620 \\
\hline 6.0 & 37,800 & 17.5 & 104,615 & 29.0 & 206,600 & 40.5 & 409,520 \\
\hline 6.5 & 40,705 & 18.0 & 107,520 & 29.5 & 215,500 & 41.0 & 418,420 \\
\hline 7.0 & 43,610 & 18.5 & 110,425 & 30.0 & 224,400 & 41.5 & 427,320 \\
\hline 7.5 & 46,515 & 19.0 & 113,330 & 30.5 & 233,300 & 42.0 & 436,220 \\
\hline 8.0 & 49,420 & 19.5 & 116,235 & 31.0 & 242,200 & 42.5 & 445,120 \\
\hline 8.5 & 52,325 & 20.0 & 119,140 & 31.5 & 251,100 & 43.0 & 454,020 \\
\hline 9.0 & 55,230 & 20.5 & 122,045 & 32.0 & 260,000 & 43.5 & 462,920 \\
\hline 9.5 & 58,135 & 21.0 & 124,950 & 32.5 & 268,900 & 44.0 & 471,820 \\
\hline 10.0 & 61,040 & 21.5 & 127,855 & 33.0 & 276,020 & 44.5 & 480,720 \\
\hline 10.5 & 63,945 & 22.0 & 130,760 & 33.5 & 284,920 & & \\
\hline
\end{tabular}

Note-This table applied to the foregoing "daily gage heights" gives the cubic feet per second flowing in the river on each date for which the gage height is given. 
Daily gage height of Tennessee River at Chattanooga, Tenn, for 1896.

\begin{tabular}{|c|c|c|c|c|c|c|c|c|c|c|c|c|}
\hline Day & an, & Feb. & Mar. & April & May & |Jun. & July & Aug. & Sept. & Oct. & Nov. & Dec \\
\hline $\begin{array}{l}3 \\
5 . \\
6 . \\
8 . \\
9 .\end{array}$ & $\begin{array}{l}4.9 \\
5.0 \\
4.9 \\
4.9 \\
4.7 \\
4.3 \\
3.6 \\
3.3 \\
3.2 \\
3.2 \\
3.1 \\
3.1 \\
2.9 \\
2.7 \\
2.6 \\
2.4 \\
2.3 \\
2.3 \\
2.3 \\
2.3 \\
2.3 \\
2.5 \\
3.1 \\
5.0 \\
6.5 \\
8.2 \\
8.0 \\
7.0 \\
6.0 \\
5.3 \\
4.8\end{array}$ & $\begin{array}{r}4.4 \\
6.2 \\
10.0 \\
11.6 \\
10.5 \\
9.3 \\
11.8 \\
14.0 \\
13.8 \\
13.2 \\
12.8 \\
11.4 \\
10.1 \\
11.1 \\
12.8 \\
13.6 \\
12.5 \\
11.0 \\
9.0 \\
7.6 \\
6.7 \\
6.0 \\
5.4 \\
4.9 \\
4.7 \\
4.6 \\
4.5 \\
4.4 \\
4.2\end{array}$ & $\begin{array}{r}4.1 \\
3.9 \\
3.8 \\
3.7 \\
3.6 \\
3.5 \\
3.4 \\
3.5 \\
3.5 \\
3.4 \\
3.6 \\
3.6 \\
3.8 \\
3.8 \\
3.7 \\
3.8 \\
5.5 \\
10.1 \\
13.1 \\
15.7 \\
13.8 \\
11.2 \\
9.5 \\
8.4 \\
7.9 \\
7.5 \\
7.2 \\
6.7 \\
6.2 \\
5.8 \\
7.7\end{array}$ & \begin{tabular}{|r|}
14.8 \\
27.7 \\
34.4 \\
38.8 \\
40.5 \\
36.9 \\
23.3 \\
11.6 \\
9.0 \\
8.0 \\
7.2 \\
6.7 \\
6.2 \\
5.8 \\
5.5 \\
5.2 \\
5.0 \\
4.8 \\
4.2 \\
4.4 \\
4.2 \\
4.1 \\
4.1 \\
4.0 \\
4.0 \\
3.8 \\
3.8 \\
3.8 \\
3.6 \\
3.6 \\
$\ldots .$.
\end{tabular} & $\begin{array}{l}3.4 \\
3.4 \\
3.4 \\
3.5 \\
4.0 \\
4.6 \\
4.6 \\
4.3 \\
4.0 \\
3.7 \\
3.4 \\
3.1 \\
2.9 \\
2.8 \\
2.7 \\
2.6 \\
2.5 \\
2.4 \\
2.4 \\
2.2 \\
2.1 \\
2.1 \\
2.5 \\
3.2 \\
3.6 \\
3.8 \\
3.2 \\
3.1 \\
2.8 \\
2.7 \\
2.5\end{array}$ & $\begin{array}{l}2.6 \\
3.0 \\
4.4 \\
5.7 \\
5.2 \\
4.7 \\
4.1 \\
3.5 \\
3.5 \\
4.5 \\
7.0 \\
6.3 \\
5.1 \\
4.3 \\
3.6 \\
3.2 \\
3.0 \\
2.8 \\
2.9 \\
3.1 \\
3.7 \\
3.5 \\
3.5 \\
3.3 \\
3.1 \\
2.9 \\
2.6 \\
2.6 \\
2.8 \\
3.0 \\
\ldots .0\end{array}$ & $\begin{array}{r}3.3 \\
3.2 \\
3.1 \\
3.2 \\
3.3 \\
3.2 \\
3.6 \\
5.0 \\
3.9 \\
14.2 \\
21.1 \\
21.6 \\
15.6 \\
11.5 \\
11.2 \\
11.4 \\
11.0 \\
13.9 \\
12.5 \\
9.6 \\
7.6 \\
6.5 \\
8.5 \\
8.8 \\
8.6 \\
7.8 \\
11.1 \\
12.2 \\
9.3 \\
7.2 \\
6.2\end{array}$ & $\begin{array}{l}5.5 \\
5.2 \\
4.8 \\
4.6 \\
4.5 \\
4.9 \\
5.0 \\
4.2 \\
3.8 \\
3.4 \\
3.3 \\
3.4 \\
3.2 \\
3.2 \\
3.1 \\
3.0 \\
3.0 \\
2.9 \\
2.7 \\
2.6 \\
2.4 \\
2.4 \\
2.2 \\
2.2 \\
2.8 \\
2.6 \\
2.7 \\
3.2 \\
4.0 \\
3.6 \\
2.8\end{array}$ & $\begin{array}{r}2.4 \\
2.1 \\
1.9 \\
1.8 \\
1.6 \\
1.6 \\
1.5 \\
2.0 \\
2.8 \\
2.7 \\
2.4 \\
2.0 \\
1.8 \\
1.6 \\
1.6 \\
1.3 \\
1.4 \\
1.4 \\
1.3 \\
1.2 \\
1.2 \\
1.4 \\
1.6 \\
2.0 \\
1.7 \\
1.5 \\
1.5 \\
2.7 \\
\ldots . .6\end{array}$ & $\begin{array}{l}2.5 \\
2.3 \\
2.6 \\
3.0 \\
2.7 \\
2.6 \\
2.1 \\
1.7 \\
1.5 \\
1.4 \\
1.2 \\
1.2 \\
1.2 \\
1.2 \\
1.5 \\
1.7 \\
1.6 \\
1.6 \\
1.6 \\
1.6 \\
1.4 \\
1.2 \\
1.2 \\
1.2 \\
1.2 \\
1.2 \\
1.2 \\
1.1\end{array}$ & 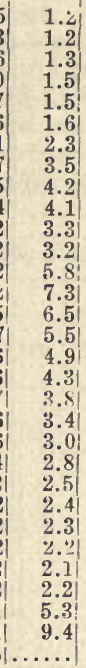 & $\begin{array}{l}2 . \\
2 . \\
2 . \\
2 . \\
2 . \\
3 . \\
2 . \\
2 . \\
2 . \\
2 . \\
4 .\end{array}$ \\
\hline
\end{tabular}

The following discharge measurements were made by Max Hall and others during 1897 :

May 8-Gage height, 7.07 feet; discharge, 44,187 second-feet. May 28-Gage neight, 4.52 feet; discharge, 25,892 second-feet. June 29-Gage height, 5.76 feet; discharge, 32,943 second-feet. July 13-Gage height, 4.59 feet; discharge, 26.884 second-feet. Sept. 7-Gage height, 1.67 feet; discharge, 10,313 second-feet. Oct. 6-Gage height, 0.48 feet; discharge, 5,969 second-feet. Nov. 16-Gage height, 0.83 feet; discharge, 5,552 second-feet. Dec. 23-Gage height, 10.30 feet; discharge, 67,000 second-feet. 
Daily gage height of Tennessee River at Chattanooga, Tenn., for 1897.

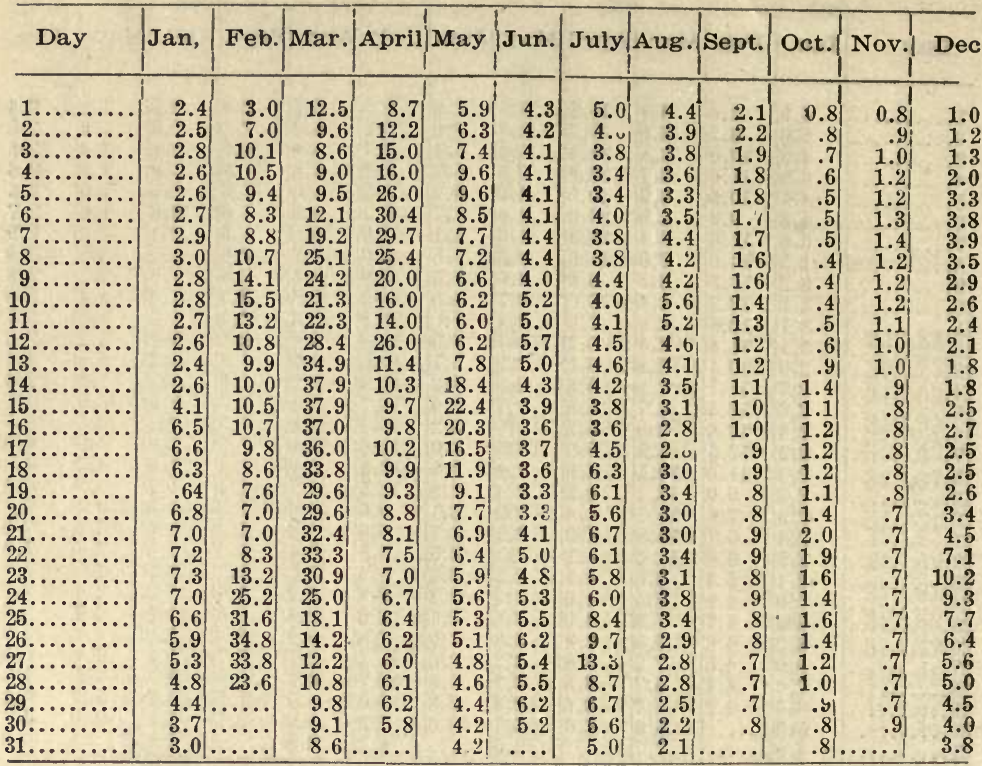

Rating table for Tennessee River at Chattanooga, Tennessee, for (a) 1896-189\%.

\begin{tabular}{|c|c|c|c|c|c|c|c|}
\hline 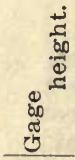 & 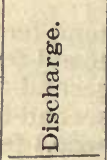 & 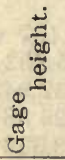 & 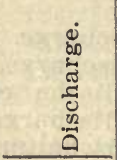 & 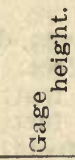 & 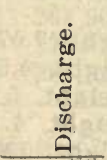 & 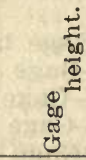 & 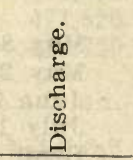 \\
\hline Fee. & Second $f t$. & Fee & Second ft. & Feet & Secmil ft & Feet. & Seeond ft. \\
\hline 0.2 & 3,080 & 1.4 & 10,208 & 3.4 & 22,088 & 12.0 & 73,172 \\
\hline 0.3 & 3,674 & 1.6 & 11,396 & 3.6 & 23,276 & 13.0 & 79,112 \\
\hline 0.4 & 4,268 & 1.8 & 12,584 & 3.8 & 24,464 & 14.0 & 85,052 \\
\hline 0.5 & 4,862 & 2.0 & 13,772 & 4.0 & 25,652 & 15.0 & 90,992 \\
\hline 0.6 & 5,456 & 2.2 & 14,960 & 4.4 & 28,028 & 16.0 & 96,932 \\
\hline 0.7 & 6,050 & 2.4 & 16,148 & 4.8 & 30,404 & 18.0 & 108,812 \\
\hline 0.8 & 6,644 & 2.6 & 17,336 & 6.0 & 37,532 & 20.0 & 120,690 \\
\hline 0.9 & 7,238 & 2.8 & 18,524 & 8.0 & 49,412 & 22.0 & 132,570 \\
\hline $1 .($ & 7,832 & 3.0 & 19,712 & 10.0 & 61,292 & 24.0 & 144,450 \\
\hline 1.2 & 9,020 & 3.2 & 20,900 & 11.0 & 67,232 & 26.0 & 156,330 \\
\hline
\end{tabular}

a Above 26 feet use table as published in the Eighteenth Ann. Rept., Part IV, p. 120.

NoTE-This table applied to the foregoing "daily gage heights" gives the cubic feet per second flowing in the river on each date for which the gage height is given. 
The following discharge measurements were made on the Tennessee River at Chattanooga, Tenn., by Max Hall and others during 1898 :

May 10-Gage height, 4.14 feet; discharge, 22,066 second-feet.

July 29-Gage height, 5.30 feet; discharge, 29,693 second-feet.

August 19-Gage height, 6.37 feet; discharge, 36,671 second-feet. Oct. 6-Gage height, 17.60 feet; discharge, 120,359 second-feet.

Oct. 28-Gage height, 6.00 feet; discharge, 35,953 second-feet.

Nov. 29-Gage meight, 4.75 feet; discharge, 29,569 second-feet.

Nov. 29-Gage height, 4.70 feet; discharge, 31,340 second-feet.

Daily gage height, in feet, of Tennessee River at Chattanooga, Tenn., for 1898.

\begin{tabular}{|c|c|c|c|c|c|c|c|c|c|c|c|c|}
\hline Day & Jan, & Feb. & Mar. & April & May & Jun. & July & Aug. & Sept. & Oct. & Nov. & Dec \\
\hline & 3.45 & 7.55 & $\mathbf{3 . 3 0}$ & 17.45 & 6.40 & 3.35 & 2.45 & 8.15 & 3.55 & 3.55 & 4.60 & \\
\hline & & & 3.15 & 17.80 & 5.80 & 3.30 & 2.35 & 7.55 & 3.95 & 3.30 & $\begin{array}{l}4.00 \\
4.30\end{array}$ & $\begin{array}{l}0.10 \\
5.05\end{array}$ \\
\hline & 3.05 & 6.15 & 3.00 & 15.00 & 5.40 & 3.30 & 2.25 & 6.45 & 9.15 & 3.20 & 4.25 & \\
\hline & 2.90 & 5.40 & 2.95 & 11.45 & 5.05 & 3.3 & 2.05 & 5.35 & 18.50 & 3.90 & 4.05 & \\
\hline & 2.75 & 5.00 & 3.30 & 10.35 & 4.70 & 2.85 & 2.35 & 6.25 & 25.00 & 8.90 & 3.90 & \\
\hline & 2.65 & 4.55 & 3.45 & 12.15 & 3.45 & 2.55 & 2.10 & 11.85 & 22.15 & 16.90 & 3.90 & \\
\hline & 2.70 & 4.45 & 3.50 & 11.60 & 4.35 & 2.35 & 2.10 & 14.65 & 15.70 & 16.50 & 4.25 & \\
\hline & 2.80 & 4.35 & 3.40 & 50301 & 4.10 & 2.20 & 2.15 & 12.55 & 11.25 & 10.75 & 4.45 & \\
\hline & 3.05 & 4.30 & 3.25 & y.30 & 2.29 & 2.15 & 2.60 & 10.15 & 9.50 & 8.80 & 4.501 & \\
\hline 0. & 3.25 & 4.15 & 3.05 & 0.20 & 4.15 & 1.95 & 3.05 & 8.50 & 8.60 & 8.40 & 4.45 & \\
\hline & 3.25 & 4.00 & 2.90 & 8. 601 & 4.45 & 1.30 & 3.50 & 9.05 & 7.45 & 7.55 & 4.65 & \\
\hline & 5.50 & 3.90 & $2.855^{\circ}$ & 9.50 & $4.65 !$ & 1.95 & 3.40 & 12.30 & 6.45 & 6.55 & 5.05 & \\
\hline & 13.20 & 3.80 & 2.80 & 2.40 & 1.411 & 1.60 & 3.20 & 14.85 & 5.70 & 6.00 & 5.30 & \\
\hline & 14.40 & 3.80 & 2.85 & 9.00 & 4.25 & 1.75 & .80 & 15.85 & 5.20 & 5.70 & 4.95 & \\
\hline & .25 & 3.80 & 3.15 & 9.05 & 3.95 & 1. 65 & .85 & 14.95 & 4.80 & 5.35 & 4.55 & \\
\hline & 12.20 & 3.70 & 5.10 & 9.15 & 3.901 & 1. 75 & .50 & 11.60 & 4.45 & 4.90 & 4.40 & \\
\hline & 12.35 & 3.55 & 5.05 & S. $60^{\circ}$ & 3.801 & 2.00 & 4 & 8.90 & 4.25 & 4.70 & 4.55 & \\
\hline & 10.00 & 3.50 & 5.20 & 3.20 & 3.70 & 2.35 & 5.35 & 7.10 & 3.95 & 5.25 & 4.75 & \\
\hline & 9.20 & 3.30 & 5.50 & 9.00 & 3.70 & 3.50 & 4.60 & 6.40 & 3.75 & 6.70 & $4.9 \mathrm{~b}$ & \\
\hline & 11.70 & 3.30 & 6.10 & 7.95 & 3.65 & 4.05 & 4.15 & 6.05 & 3.55 & 7.75 & 5.30 & \\
\hline & 13.80 & 3.20 & 5.70 & 7.40 & 8.30 & 5.3 & 4.00 & 5.95 & 3.4 & 9.30 & 5.85 & \\
\hline & 13.40 & 3.25 & 5.45 & 7.05 & $3.6 ; 0)$ & 5.55 & 3.30 & 5.65 & 3.55 & & 6.05 & \\
\hline & 12.55 & 3.40 & 5.15 & 6. $f 0$ & 3.50 & 5.05 & 3.30 & 5.40 & 5.00 & 7.65 & 6.55 & \\
\hline & 12.35 & 3.50 & 4.65 & 6.5 & 3.40 & 4.55 & 3.40 & 4.75 & 5.05 & 7.25 & 6.85 & \\
\hline & 12.35 & 3.60 & 4.30 & 6.65 & 3.4 & 3.70 & 3.55 & 4.30 & 6.4 & 7.20 & 6.55 & \\
\hline & 16.0 & 3.8 & 4.15 & 5.3 & 3.701 & & 3.55 & 4.05 & 7.20 & 7.6 & 6.10 & \\
\hline & 18.20 & 3.5 & 4.45 & 6.45 & 4.95 & 2.90 & 4.55 & 4.00 & 6.15 & 6.90 & 5.65 & \\
\hline & 16.70 & 3.35 & 4.45 & 7.29 & 5.60 & 2.80 & 5.55 & 4.10 & 5.00 & 6.20 & 5.15 & \\
\hline & 14.15 & & 4.65 & 7,05 & 4.97 & 2.80 & 5.35 & 4... & 4.30 & 5.65 & 4.80 & 5.15 \\
\hline & 11.20 & & 5.55 & 5.75 & 4. 50? & 2.55 & 5.90 & 4.10 & 3.85 & 5.15 & 4.95 & \\
\hline & 8.95 & & & & 3.70 & & 7.90 & 3.85 & ....... & 4.85 & .....। & \\
\hline
\end{tabular}


Rating table for Tennessee River at Chattanooga, Tenn., for 1898.

\begin{tabular}{|c|c|c|c|c|c|c|c|}
\hline 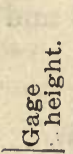 & 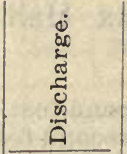 & 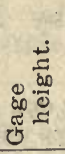 & 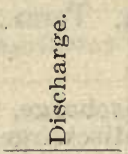 & 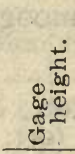 & 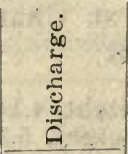 & 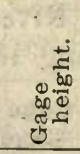 & 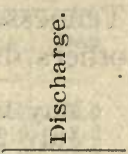 \\
\hline Feet. & Second $f l$. & Feet. & Second feet. & Feet. & Second feet. & Feet. & Second feet. \\
\hline 0.5 & 5,900 & 3.7 & 19,550 & 6.9 & 42,370 & 10.5 & 68,650 \\
\hline 0.6 & 6,266 & 3.8 & 20,120 & 7.0 & 43,100 & 11.0 & 72,300 \\
\hline 0.7 & 6,634 & 3.9 & 20,700 & 7.1 & 43,830 & 11.5 & 75,950 \\
\hline 0.8 & 7,004 & 4.0 & 21,320 & 7.2 & 44,560 & 12.0 & 79,600 \\
\hline 0.9 & 7,376 & 4.1 & 21,950 & 7.3 & 45,290 & 12.5 & 83,250 \\
\hline 1.0 & 7,750 & 4.2 & 22,580 & 7.4 & 46,020 & 13.0 & 86,900 \\
\hline 1.1 & 8,126 & 4.3 & 23,350 & 7.5 & 46,750 & 13.5 & 90,550 \\
\hline 1.2 & 8,504 & 4.4 & 24,120 & 7.6 & 47,480 & 14.0 & 94,200 \\
\hline 1.3 & 8,884 & 4.5 & 24,850 & 7.7 & 48,210 & 14.5 & 97,850 \\
\hline 1.4 & 9,266 & 4.6 & 25,580 & 7.8 & 48,940 & 15.0 & 101,500 \\
\hline 1.5 & 9,650 & 4.7 & 26,310 & 7.9 & 49,670 & 15.5 & 105,150 \\
\hline 1.6 & 10,046 & 4.8 & 27,040 & 8.0 & 50,400 & 16.0 & 108,800 \\
\hline 1.7 & 10,444 & 4.9 & 27,770 & 8.1 & 51,130 & 16.5 & 112,450 \\
\hline 1.8 & 10,844 & 5.0 & 28,500 & 8.2 & 51,860 & 17.0 & 116,100 \\
\hline 1.9 & 11,246 & 5.1 & 29,230 & 8.3 & 52,590 & 17.5 & 119,750 \\
\hline 2.0 & 11,650 & 5.2 & 29,960 & 8.4 & 53,320 & 18.0 & 123,400 \\
\hline 2.1 & 12,056 . & 5.3 & 30,690 & 8.5 & 54,050 & 18.5 & 127,050 \\
\hline 2.2 & 12,464 & 5.4 & 31,420 & 8.6 & 54,780 & 19.0 & 130,700 \\
\hline 2.3 & 12,874 & 5.5 & 32,150 & 8.7 & 55,510 & 19.5 & 134,350 \\
\hline 2.4 & 13,286 & 5.6 & 32,880 & 8.8 & 56,240 & 20.0 & 138,000 \\
\hline 2.5 & 13,700 & 5.7 & 33,610 & 8.9 & ๖6,970 & 20.5 & 141,650 \\
\hline 2.6 & 14,126 & 5.8 & 34,340 & 9.0 & 57,700 & 21.0 & 145,300 \\
\hline 2.7 & 14,562 & 5.9 & 35,070 & 9.1 & 58,430 & 21.5 & 148,950 \\
\hline 2.8 & 15,008 & 6.0 & 35,800 & 9.2 & 59,160 & 22.0 & 152,600 \\
\hline 2.9 & 15,464 & 6.1 & 36,530 & 9.3 & 59,890 & 22.5 & 156,250 \\
\hline 3.0 & 15,930 & 6.2 & 37,260 & 9.4 & 60,620 & 23.0 & 159,900 \\
\hline 3.1 & 16,410 & 6.3 & 37,990 & 9.5 & 61,350 & 23.5 & 163,550 \\
\hline 3.2 & 16,900 & 6.4 & 38,720 & 9.6 & 62,080 & 24.0 & 167,200 \\
\hline 3.3 & 17,400 & 6.5 & 39,450 & 9.7 & 62,810 & 24.6 & 171,680 \\
\hline 3.4 & 17,920 & 6.6 & 40,180 & 9.8 & 63,540 & & \\
\hline 3.5 & 18,460 & 6.7 & 40,910 & 9.9 & 64,270 & & \\
\hline 3.6 & 19,000 & 6.8 & 41,640 & 10.0 & 65,000 & & \\
\hline
\end{tabular}

Note-This table applied to the foregoing "daily gage heights" gives the cubic feet per second flowing in the river on each date for which the gage height is given.

The following measurements. were made by Max Hall and others during 1899 :

May 3-Gage height, 6.71 feet; discharge, 37,770 second-feet.

May 26-Gage height, 4.76 feet; discharge, 25,526 second-feet.

June 21-Gage height, 4.15 feet; discharge, 21.391 second-feet.

Sept. 15-Gage height, 1.90 feet; discharge, 10,819 second-feet.

Oct. 27-Gage height, 0.80 foot; discharge, 6,566 second-feet. 
Daily gage height in feet of Tennessee River at Chattanooga, Tenn., for 1899.

\begin{tabular}{|c|c|c|c|c|c|c|c|c|c|c|c|c|}
\hline Day & Jan. & Feb. & Mar. & April & May & Jun. & July & ug. & ept.| & & & Dee. \\
\hline & 4.75 & 5.70 & 19.25 & 22.80 & 7.601 & 4.15 & 3.45 & 4.20 & 2.20 & 1.20 & 1.10 & 1.70 \\
\hline & & 5.65 & 17.60 & 19.50 & 7.10 & 4.25 & 3.30 & 3,55 & 2.35 & 1.10 & 1.10 & 1.70 \\
\hline & 5.30 & 5.60 & 15.15 & 14.90 & 6.70 & 4.4 & 3.05 & 3.05 & 2.80 & 1.05 & & 1.70 \\
\hline & 5.80 & 10.70 & 14.15 & 12.95 & 6.30 & 4.8 & 2.80 & 2.75 & 3.05 & .95 & 1.10 & 1.70 \\
\hline & 5.95 & 23.10 & 17.95 & 13.25 & 6.15 & 4.6 & 2.60 & 2.45 & 2.65 & .90 & 50 & 1.80 \\
\hline & 7.25 & 30.45 & 24.50 & 14.70 & 7.10 & 4.2 & 2.60 & 2.45 & 2.25 & .80 & 1.50 & 1.70 \\
\hline & 18.80 & 34.30 & 26.05 & 15.70 & $8.5 C$ & 4. & 2.65 & 2.40 & 1.95 & .85 & 1.5 & 1.6 \\
\hline & 18.40 & 36.95 & 27.60 & 18.05 & 9.35 & 3.7 & 3.05 & 2.40 & 1.80 & 1.00 & 1.45 & 1.50 \\
\hline & $17.3 \mathrm{~b}$ & 38.20 & 27.70 & 17.75 & 10.00 & & 2.90 & 2.25 & 1.60 & 1.15 & 1.35 & 1.4 \\
\hline & .15 & 36.75 & 16.15 & 15.70 & $10.7 \mathrm{C}$ & 3.40 & 2.60 & 2.10 & 1.80 & 1.60 & 1.20 & 1.40 \\
\hline & 13.85 & 30.30 & 11.85 & 14.20 & 11.15 & 3. & 2.60 & 2.10 & 1.70 & 1.80 & 1.15 & 1. \\
\hline & 10.50 & 19.35 & 10.60 & 12.90 & 10.40 & 4.30 & 2.45 & 2.00 & 2.00 & 1.85 & 1. & 5.20 \\
\hline & 9.15 & 12.15 & 9.55 & 11.65 & 9.60 & 5. & 2.30 & 2.00 & 1.80 & 1.70 & 1.00 & 6.45 \\
\hline & .10 & 9.50 & 11.20 & 10.70 & 9.30 & 5.8 & 2.20 & 2.25 & 2.00 & 1.65 & & 7. \\
\hline & & 8.50 & 24.55 & 10.00 & 9.55 & 6.4 & 2.15 & 2.65 & 1.85 & 1.40 & 1.00 & 7.15 \\
\hline & 7.30 & 7.55 & 34.25 & 9.40 & 9.20 & 6. & 1.95 & 2.65 & 1.65 & 1.25 & 1. & 6. \\
\hline & 7.40 & 7.95 & 36.90 & 8.75 & 8.70 & 6.4 & 1.90 & $2.40 !$ & 1.45 & 1.15 & 1.00 & 5.2 \\
\hline & 7.45 & 9.55 & 36.15 & 8.40 & 7.75 & 6.2 & 1.80 & 2.30 & 1.35 & 1.15 & 1.00 & 4.25 \\
\hline & 7.25 & 11.30 & 35.85 & 8.00 & 6.90 & 5.2 & 1.90 & 2.15 & 1.20 & 1.10 & 1.00 & 3.8 \\
\hline & 7.00 & 12.65 & 37.05 & 7.55 & 6.40 & 4.7 & 2.05 & 1.90 & 1.05 & 1.10 & .95 & 4.25 \\
\hline & 6.8 & 11.50 & 39.20 & 7.35 & 5.90 & 4.2 & 2.05 & 1.70 & 1. & 1.10 & .85 & 4.40 \\
\hline & 6.45 & 10.65 & 40.00 & 7.05 & 5.60 & 3.7 & 2.40 & 1.60 & 1.05 & 1.10 & .85 & 4.40 \\
\hline & 5.90 & 10.10 & 38.70 & 7.85 & 5.35 & & 2.70 & 1.45 & 1.30 & 1.05 & 1.00 & 4.15 \\
\hline & 5.65 & 9.75 & 32.70 & 9.65 & 5.30 & 3.25 & 3.50 & 1.30 & 1.50 & 1.00 & 1.15 & 5.65 \\
\hline .. & 6.05 & 9.50 & 23.15 & 9.35 & 5.05 & 3.15 & 3.40 & 1.20 & 1.50 & .95 & 1.301 & fi. 15 \\
\hline & 6.35 & 9.20 & 16.30 & 10.75 & 4.80 & 3.00 & 3.00 & 1.20 & 1.45 & .851 & $1.70 \mid$ & 6.30 \\
\hline & 5.85 & 13.20 & 13.65 & 10.30 & 4.65 & 3.25 & 3.05 & 1.20 & 1.30 & .80 & 1.80 & 5.85 \\
\hline & 5.75 & 18.45 & 13.95 & 9.20 & 4.40 & 3.65 & 3.65 & $1.2 \mathrm{~b}$ & 1.20 & .80 & 1.85 & 5.55 \\
\hline & 5.5 & & 17.30 & 8.35 & 4.30 & 3.50 & 4.25 & 1.50 & 1.25 & .90 & 1.80 & 5.10 \\
\hline & & & 21.20 & 7.75 & 4.20 & 3.30 & 4.20 & 1.85 & 1.30 & 1.00 & 1.75 & 4.65 \\
\hline & & & $|22.80|$ & & 4.25 & $\ldots$ & 5.10 & 1.75 & $1 \cdots \cdots \cdots$ & 1.05 & ....... & 3.85 \\
\hline
\end{tabular}

During 1900 the following measurements were made by Max Hall and others :

March 13-Gage height, 11.25 feet; discharge, 66,012 secod-feet.

July 27-Gage height, 3.45 feet; discharge, 18,470 second-feet. 
Daily gage height in feet of Tennessee River at Chattanooga, Tenn., for 1900.

\begin{tabular}{|c|c|c|c|c|c|c|c|c|c|c|c|c|}
\hline $\mathrm{Da}$ & an, & Feb. & Mar. & April & May & Jun. & July & ug. & Sept. & Oct. & Nov. & De \\
\hline & & & .05 & .85 & 6.20 & 2.85 & 8.85 & 6.20 & .10 & 2.00 & 2.90 & \\
\hline & & & .70 & .20 & & 2.80 & 8.15 & 5.40 & & 1.80 & & \\
\hline & * & & 0.90 & 6.85 & & 3.00 & & 70 & & & & \\
\hline & & & 12.50 & 7.25 & 5 & 3.20 & 30 & .20 & & & & \\
\hline & & & .75 & 8.05 & & 3.20 & & 60 & & & & \\
\hline & 2.10 & 3. & .65 & 8.55 & & 3.50 & .40 & .20 & & & 3.70 & \\
\hline & & 3. & 0.00 & 7.85 & & 5.6 & & 90 & & & & \\
\hline & & 3. & 11.65 & 7.05 & & 6.6 & .50 & 60 & & & & \\
\hline & & 5. & 14.55 & 6.50 & & & & & & & & \\
\hline & & 8.4 & 16.50 & 6.10 & & 5.30 & .20 & $30 \mid$ & & & 3.20 & \\
\hline & & & 16.15 & & & 5. & & & & & & \\
\hline & & 8. & 14.2 & & & 4.90 & 30 & .00 & & & & \\
\hline & & 13.90 & 11.65 & 7.40 & & 4.5 & & & & & & \\
\hline & & 21 & 9.8 & & & & & & & & & \\
\hline & & 2 & & 6.5 & & 5. & & & & & & \\
\hline & & & 8.00 & 6.30 & & 5. & & .20 & & & 2.10 & \\
\hline & & 17.0 & 7.80 & 8.75 & & 5. & & & & & & \\
\hline & & 12. & 7.55 & 10.65 & & & & 30 & & (1) & & \\
\hline & & 9. & 7.55 & 9.75 & & 8.8 & & .30 & & & & \\
\hline & & 7.70 & 8.55 & & & & & & & & & \\
\hline & & 7.10 & 11.60 & 11.7 & & 8.5 & & .90 & & & & \\
\hline & & 7.7 & 14.95 & & & 7. & & 1.80 & & & & \\
\hline & & 8.5 & 17.4 & & & 6. & & 1.7 & & & & \\
\hline & & 8.5 & 16.45 & 10.70 & & 6. & & & & & & \\
\hline & & 8.5 & 12. & & & 7. & & & & & & \\
\hline & & 9. & 11.1 & & & 7. & & & & & & \\
\hline & & 9. & 10.9 & & & 8. & & & & & & \\
\hline & & 8.45 & $10 . ?$ & 7.4 & 3. & 8. & & & & & & \\
\hline & & & 10.20 & 7.0 & & 8.60 & & 2.5 & & & & \\
\hline & & & 9.35 & 6.60 & 3. & 8.70 & & 2.30 & 2.20 & 3.70 & 13.20 & \\
\hline & & & & & 3.05 & & 7.30 & 2,20 & $|\ldots \ldots|$. & $3.40 \mid$ & $\ldots \ldots$ & 4.5 \\
\hline
\end{tabular}

*Frozen at gage. 
Rating table for Tennessee River at Chattanooga, Tennessee, for 1899-1900.

\begin{tabular}{|c|c|c|c|c|c|c|c|}
\hline 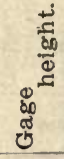 & 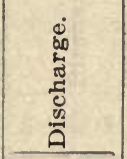 & 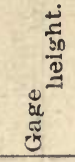 & 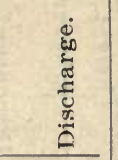 & & 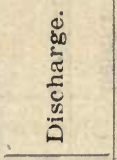 & 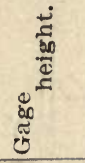 & 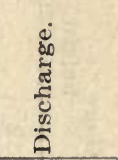 \\
\hline Feel. & SSecond $f i$. & Beet. & Second $f t$. & Feet- & Second $f t$ & Feet. & Sicond $f t i$ \\
\hline 0.8 & 6,600 & 10.7 & 62,340 & 20.6 & 123,720 & 30.5 & 185,100 \\
\hline 0.9 & 6,950 & 10.8 & 62,960 & 20.7 & 124,340 & 30.6 & 185,720 \\
\hline 1.0 & 7,300 & 10.9 & 63,580 & 20.8 & 124,960 & 30.7 & 186,340 \\
\hline 1.1 & 7,670 & 11.0 & 64,200 & 20.9 & 125,580 & 30.8 & 186,960 \\
\hline 1.2 & 8,040 & 11.1 & 64,820 & 21.0 & 126,200 & 30.9 & 187,580 \\
\hline 1.3 & 8,430 & 11.2 & 65,440 & 21.1 & 126,820 & 31.0 & 188,200 \\
\hline 1.4 & 8,820 & 11.3 & 66,060 & 21.2 & 127,440 & 31.1 & 188,820 \\
\hline 1.5 & 9,220 & 11.4 & 66,680 & 21.3 & 128,060 & 31.2 & 189,440 \\
\hline 1.6 & 9,620 & 11.5 & 67,300 & 21.4 & 128,680 & 31.3 & 190,060 \\
\hline 1.7 & 10,020 & 11.6 & 67,920 & 21.5 & 129,300 & 31.4 & 190,680 \\
\hline 1.8 & 10,430 & 11.7 & 68,540 & 21.6 & 129,920 & 31.5 & 191,300 \\
\hline 1.9 & 10,840 & 11.8 & 69,160 & 21.7 & 130,540 & 31.6 & 191,920 \\
\hline 2.0 & 11,250 & 11.9 & 69,780 & 21.8 & 131,160 & 31.7 & 192,540 \\
\hline 2.1 & 11,660 & 12.0 & 70,400 & 21.9 & 131,780 & 31.8 & 193,160 \\
\hline 2.2 & 12,080 & 12.1 & 71,020 & 22.0 & 132,400 & 21.9 & 193,780 \\
\hline 2.3 & 12,500 & 12.2 & 71,640 & 22.1 & 133,020 & 32.0 & 194,400 \\
\hline 2.4 & 12,930 & 12.3 & 72,260 & 22.2 & 133,640 & 32.1 & 195,020 \\
\hline 2.5 & 13,360 & 12.4 & 72,880 & 22.3 & 134,260 & 32.2 & 195,640 \\
\hline 2.6 & 13,800 & 12.5 & 73,500 & 22.4 & 134,880 & 32.3 & 196,260 \\
\hline 2.7 & 14,240 & 12.6 & 74,120 & 22.5 & 135,500 & 32.4 & 196,880 \\
\hline 2.8 & 14,680 & 12.7 & 74,740 & 22.6 & 136,120 & 32.5 & 197,500 \\
\hline 2.9 & 15,140 & 12.8 & 75,360 & 22.7 & 136,740 & 32.6 & 198,120 \\
\hline 3.0 & 15,600 & 12.9 & 75,980 & 22.8 & 137,360 & 32.7 & 198,740 \\
\hline 3.1 & 16,080 & 13.0 & 76,600 & 22.9 & 137,980 & 32.8 & 199,360 \\
\hline 3.2 & 16,550 & 13.1 & 77,220 & 23.0 & 138,600 & 32.9 & 199,980 \\
\hline 3.3 & 17,050 & 13.2 & 77,840 & 23.1 & 139,220 & 33.0 & 200,600 \\
\hline 3.4 & 17,550 & 13.3 & 78,460 & 23.2 & 139,840 & 33.1 & 201,220 \\
\hline 3.5 & 18,050 & 10.4 & 79,080 & 23.3 & 140,460 & 33.2 & 201,840 \\
\hline 3.6 & 18,550 & 13.5 & 79,700 & 23.4 & 141,080 & 33.3 & 202,460 \\
\hline 3.7 & 19,050 & 13.6 & 80,320 & 23.5 & 141,700 & 33.4 & 203,080 \\
\hline 3.8 & 19,600 & 13.7 & 80,940 & 23.6 & 142,320 & 33.5 & 203,700 \\
\hline 3.9 & 20,200 & 13.8 & 81,560 & 23.7 & 142,940 & 33.6 & 204,320 \\
\hline 4.0 & 20,800 & 13.9 & 82,180 & 23.8 & 143,560 & 33.7 & 204,940 \\
\hline 4.1 & 21,420 & 14.0 & 82,800 & 23.9 & 144,180 & 33.8 & 205,560 \\
\hline 4.2 & 22,040 & 14.1 & 83,420 & 24.0 & 144,800 & 33.9 & 206,180 \\
\hline 4.3 & 22,660 & 14.2 & 84,040 & 24.1 & 145,420 & 34.0 & 206,800 \\
\hline 4.4 & 23,280 & 14.3 & 84,660 & 24.2 & 146,040 & 34.1 & 207,420 \\
\hline 4.5 & 23,900 & 14.4 & 85,280 & 24.3 & 146,660 & 34.2 & 208,040 \\
\hline 4.6 & 24,520 & 14.5 & 85,900 & 24.4 & 147,280 & 34.3 & 208,660 \\
\hline 4.7 & 25,140 & 14.6 & 86,520 & 24.5 & 147,900 & 34.4 & 209,280 \\
\hline 4.8 & 25,760 & 14.7 & 87,140 & 24.6 & 148,520 & 34.5 & 209,900 \\
\hline 4.9 & 26,380 & 14.8 & 87,760 & 24.7 & 149,140 & 34.6 & 210,520 \\
\hline 5.0 & 27.000 & 14.9 & 88,380 & 24.8 & 149,760 & 34.7 & 211,140 \\
\hline 5.1 & 27,620 & 15.0 & 89,000 & 24.9 & 150,380 & 34.8 & 211,760 \\
\hline 5.2 & 28,240 & 15.1 & 89,620 & 25.0 & 151,000 & 34.9 & 212,380 \\
\hline 5.3 & 28,860 & 15.2 & 90,240 & 25.1 & 151,620 & 35.0 & 213,000 \\
\hline 5.4 & 29,480 & 15.3 & 90,860 & 25.2 & 152,240 & 35.1 & 213,620 \\
\hline 5.5 & 30,100 & 15.4 & 91,480 & 25.3 & 152,860 & 35.2 & 214,240 \\
\hline 5.6 & 30,720 & 15.5 & 92,100 & 25.4 & 153,480 & 35.3 & 214,860 \\
\hline
\end{tabular}


Rating table for Tennessee River at Chattanooga, Tennessee, for 1899-1900.

\begin{tabular}{|c|c|c|c|c|c|c|c|}
\hline 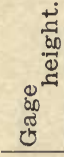 & 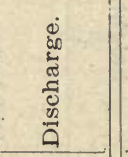 & 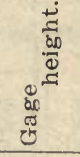 & 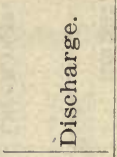 & 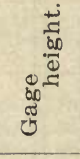 & 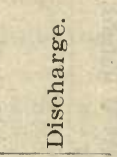 & 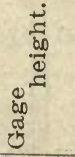 & 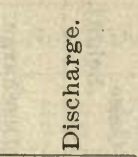 \\
\hline Feet. & Second ft. & Feel. & Second fit. & Feet, & Second $f t$. & Feet. & Second ft. \\
\hline 5.7 & 31,340 & 15.6 & 92,720 & 25.5 & 154,100 & 35.4 & 215,480 \\
\hline 5.8 & 31,960 & 15.7 & 93,340 & 25.6 & 154,720 & 35.5 & 216,100 \\
\hline 5.9 & 32,580 & 15.8 & 93,960 & 25.7 & 155,340 & 35.6 & 216,720 \\
\hline 6.0 & 33,200 & 15.9 & 94,580 & 25.8 & 155,960 & 35.7 & 207,340 \\
\hline 6.1 & 33,820 & 16.0 & 95,200 & 25.9 & 156,580 & 35.8 & 217,960 \\
\hline 6.2 & 34,440 & 16.1 & 95,820 & 26.0 & 157,200 & 35.9 & 218,580 \\
\hline 6.3 & 35,060 & 16.2 & 96,440 & 26.1 & 159,820 & 36.0 & 219,200 \\
\hline 6.4 & 35,680 & 16.3 & 97,060 & 26.2 & 158,440 & 36.1 & 219,820 \\
\hline 6.5 & 36,300 & 16.4 & 97,680 & 26.3 & 159,060 & 36.2 & 220,440 \\
\hline 6.6 & 36,920 & 16.5 & 98,300 & 26.4 & 159,680 & 36.3 & 221,060 \\
\hline 6.7 & 37,540 & 16.6 & 98,920 & 26.5 & 160,300 & 36.4 & 221,680 \\
\hline 6.8 & 38,160 & 16.7 & 99,540 & 26.6 & 160,920 & 36.5 & 222,300 \\
\hline 6.9 & 38,780 & 16.8 & 100,160 & 26.7 & 161,540 & 36.6 & 222,920 \\
\hline 7.0 & 39,400 & 16.9 & 100,780 & 26.8 & 162,160 & 36.7 & 223,540 \\
\hline 7.1 & 40,020 & 17.0 & 101,400 & 26.9 & 162,780 & 36.8 & 224,160 \\
\hline 7.2 & 40,640 & 17.1 & 102,020 & 27.0 & 163,400 & 36.9 & 224,780 \\
\hline 7.3 & 41,260 & 17.2 & 102,640 & 27.1 & 164,020 & 37.0 & 225,400 \\
\hline 7.4 & 41,380 & 17.3 & 103,260 & 27.2 & 164,640 & 37.1 & 226,020 \\
\hline 7.5 & 42,500 & 17.4 & 103,880 & 27.3 & 165,260 & 37.2 & 226,640 \\
\hline 7.6 & $43,120^{\dagger}$ & 17.5 & 104,500 & 27.4 & 165,880 & 37.3 & 227,260 \\
\hline 7.7 & 43,740 & 17.6 & 105,120 & 27.5 & 166,500 & 37.4 & 227,880 \\
\hline 7.8 & 44,360 & 17.7 & 105,740 & 27.6 & 167,120 & 37.5 & 228,500 \\
\hline 7.9 & 44,980 & 17.8 & 106,360 & 27.7 & 167,740 & 37.6 & 229.120 \\
\hline 8.0 & 45,600 & 17.9 & 106,980 & 27.8 & 168,360 & 37.7 & 229,740 \\
\hline 8.1 & 46,220 & 18.0 & 107,600 & 27.9 & 168,980 & 37.8 & 230,360 \\
\hline 8.2 & 46,840 & 18.1 & 108,220 & 28.0 & 169,600 & 37.9 & 230,980 \\
\hline 8.3 & 47,460 & 18.2 & 108,840 & 28.1 & 170,220 & 18.0 & 231,600 \\
\hline 8.4 & 48,080 & 18.3 & 109,460 & 28.2 & 170,840 & 38.1 & 232,220 \\
\hline 8.5 & 48,700 & 18.4 & 110,080 & 28.3 & 171,460 & 38.2 & 232,840 \\
\hline 8.6 & 49,320 & 18.5 & 110,700 & 28.4 & 172,080 & 38.3 & 233,460 \\
\hline 8.7 & 49,940 & 18.6 & 111,320 & 28.5 & 172,700 & 38.4 & 234,080 \\
\hline 8.8 & 50,560 & 18.7 & 111,940 & 28.6 & 173,320 & 38.5 & 234,700 \\
\hline 8.9 & 51,180 & 18.8 & 112,560 & 28.7 & 173,940 & 38.6 & 235,320 \\
\hline 9.0 & 51,800 & 18.9 & 113,180 & 28.8 & 174,560 & 38.7 & 235,940 \\
\hline 9.1 & 52,420 & 19.0 & 113,800 & 28.9 & 175,180 & 38.8 & 236,560 \\
\hline 9.2 & 53,040 & 19.1 & 114,420 & 29.0 & 175,800 & 38.9 & 237,180 \\
\hline 9.3 & $53,660^{\dagger}$ & 19.2 & 115,040 & 29.1 & 176,420 & 39.0 & 237,800 \\
\hline 9.4 & 54,280 & 19.3 & 115,660 & 29.2 & 177,040 & 39.1 & 238,420 \\
\hline 9.5 & 54,300 & 19.4 & 116,280 & 29.3 & 177,660 & 39.2 & 239,040 \\
\hline 9.6 & 55,520 & 19.5 & 116,900 & 29.4 & 178,280 & 39.3 & 239,660 \\
\hline 9.7 & 56,140 & 19.6 & 117,520 & 29.5 & 178,900 & 39.4 & 240,280 \\
\hline 9.8 & 56,760 & 19.7 & 118,140 & 29.6 & 179,520 & 39.5 & 240,900 \\
\hline 9.9 & 57,380 & 19.8 & 118,760 & 29.7 & 180,140 & 39.6 & 241.520 \\
\hline 10.0 & 58,000 & 19.9 & 119,380 & 29.8 & 180,760 & 39.7 & 242,140 \\
\hline 10.1 & 58,620 & 20.0 & 120,000 & 29.9 & 181,380 & 39.8 & 242,760 \\
\hline 10.2 & 59,240 & 20.1 & 120,620 & 30.0 & 182,000 & 39.9 & 243,380 \\
\hline 10.3 & 59,860 & 20.2 & 121,240 & 30.1 & 182,620 & 40.0 & 244,000 \\
\hline
\end{tabular}




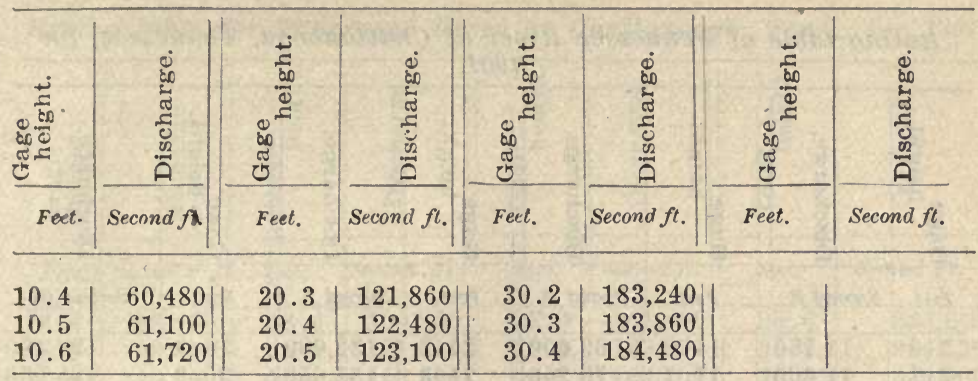

Note-This table applied to the foregring "daily gage heights" gives the cubic feet per second flowing in the river on each date for which the gage height is given.

The following discharge measurements were made on the Tennessee River at Chattanooga, Tenn., during Igo' :

1901.

Jan. 24-Hydrographer, Max Hall: Gage height, 5.60 feet; discharge, 30.317 second-feet.

April 4-Hydrographer, K. T. Thomas: Gage height, 24.20 feet; discharge, 155,457 second-feet.

July 31-Hydrographer, K. T. Thomas: Gage height, 2.80 feet; discharge, 15,393 second-feet.

Aug. 18-Hydrographer, K. T. Thomas: Gage height, 31.70 feet; discharge, 198,718 second-feet.

Daily gage height of Tennessee River at Chattanooga, Tenn., for 1901.

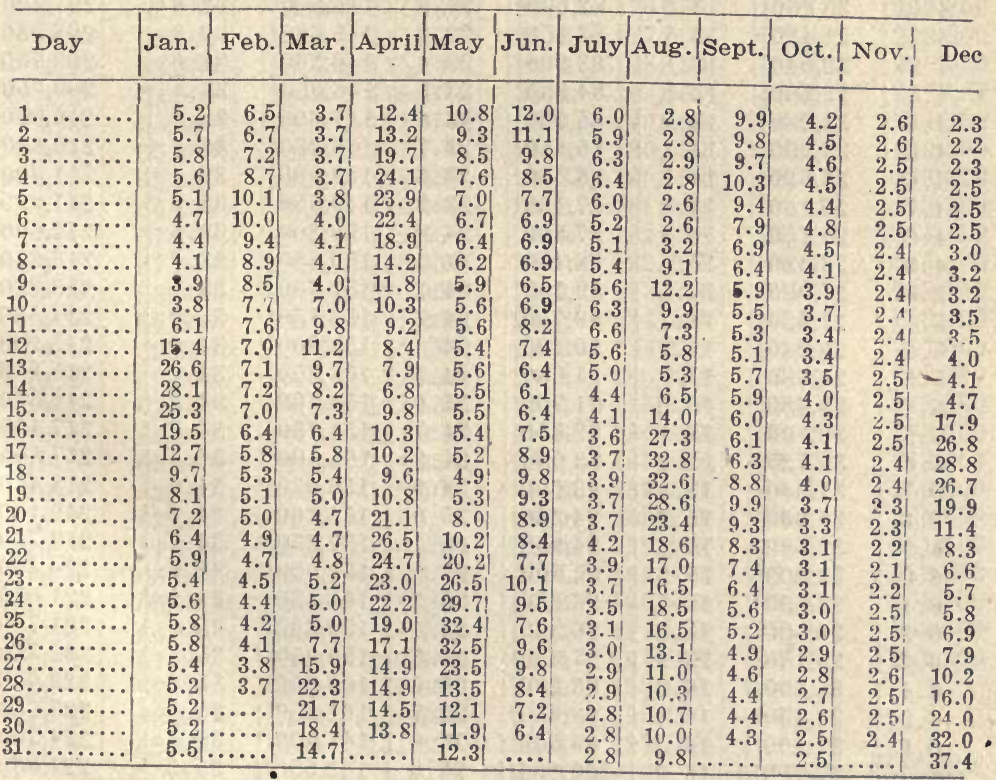


Rating table of Tennessee River at Chattanooga, Tennessee, for 1901.

\begin{tabular}{|c|c|c|c|c|c|c|c|}
\hline 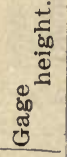 & 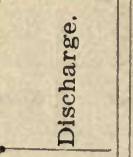 & 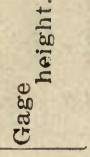 & 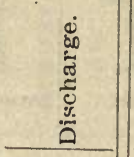 & & 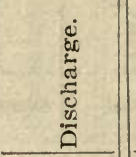 & 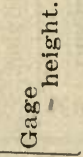 & 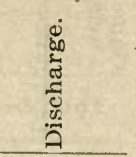 \\
\hline Feet. & Second ft. & Feet. & Seoond $f t$. & Feet. & Second $f i$. & Feet. & Second $f t$ \\
\hline 2.0 & 11,250 & 11.6 & 69,600 & 21.2 & 132,000 & 30.8 & 194,400 \\
\hline 2.1 & 11,660 & 11.7 & 70,250 & 21.3 & 132,650 & 30.9 & 195,050 \\
\hline 2.2 & 12,080 & 11.8 & 70,900 & 21.4 & 133,300 & 31.0 & 195,700 \\
\hline 2.3 & 12,500 & 11.9 & 71,550 & 21.5 & 133,950 & 31.1 & 196,350 \\
\hline 2.4 & 12,930 & 12.0 & 72,200 & 21.6 & 134,600 & 31.2 & 197,000 \\
\hline 2.5 & 13,360 & 12.1 & 72,850 & 21.7 & 135,250 & 31.3 & 197,650 \\
\hline 2.6 & 13,800 & 12.2 & 73,500 & 21.8 & 135,900 & 31.4 & 198,300 \\
\hline 2.7 & 14,240 & 12.3 & 74,150 & 21.9 & 136,550 & 31.5 & 198,950 \\
\hline 2.8 & 14,680 & 12.4 & 74,800 & 22.0 & 137,200 & 31.6 & 199,600 \\
\hline 2.9 & 15,140 & 12.5 & 75,450 & 22.1 & 137,850 & 31.7 & 200,250 \\
\hline 3.0 & 15,600 & 12.6 & 76,100 & 22.2 & 138,500 & 31.8 & 200,900 \\
\hline 3.1 & 16,080 & 12.7 & 76,750 & 12.3 & 139,150 & 31.9 & 201.550 \\
\hline 3.2 & 16,550 & 12.8 & 77,400 & 22.4 & 139,800 & 32.0 & 202,200 \\
\hline 3.3 & 17,050 & 12.9 & 78,050 & 22.5 & 140,450 & 32.1 & 202,850 \\
\hline 3.4 & 17,550 & 13.0 & 78,700 & 22.6 & 141,100 & 32.2 & 203,500 \\
\hline 3.5 & 18,050 & 13.1 & 79,350 & 22.7 & 141,750 & 32.3 & 204,150 \\
\hline 3.6 & 18,550 & 13.2 & 80,000 & 22.8 & 142,400 & 32.4 & 204,800 \\
\hline 3.7 & 19,050 & 13.3 & 80,650 & 22.9 & 143,050 & 32.5 & 205,450 \\
\hline 3.8 & 19,600 & 13.4 & 81,300 & 23.0 & 143,700 & 32.6 & 206,100 \\
\hline 3.9 & 20,200 & 13.5 & 81,950 & 23.1 & 144,350 & 32.7 & 206,750 \\
\hline 4.0 & 20,800 & 13.6 & 82,600 & 23.2 & 145,000 & 32.8 & 207,400 \\
\hline 4.1 & 21,420 & 13.7 & 83,250 & 23.3 & 145,650 & 32.9 & 208,050 \\
\hline 4.2 & 22,040 & 13.8 & 83,900 & 23.4 & 146,300 & 33.0 & 208,700 \\
\hline 4.3 & 22,660 & 13.9 & 84,550 & 23.5 & 146,950 & 33.1 & $209,{ }^{2} 50$ \\
\hline 4.4 & 23,280 & 14.0 & 85,200 & 23.6 & 147,600 & 33.2 & 210,000 \\
\hline 4.5 & 23,900 & 14.1 & 85,850 & 23.7 & 148,250 & 33.3 & 210,650 \\
\hline 4.6 & 24,520 & 14.2 & 86,500 & 23.8 & 148,900 & 33.4 & 211,300 \\
\hline 4.7 & 25,140 & 14.3 & 87,150 & 23.9 & 149,550 & 33.5 & 211,950 \\
\hline 4.8 & 25,760 & 14.4 & 87,800 & 24.0 & 150,200 & 33.6 & 212,600 \\
\hline 4.9 & 26,380 & 14.5 & 88,450 & 24.1 & 150,850 & 33.7 & 213,250 \\
\hline 5.0 & 27,000 & 14.6 & 89,100 & 24.2 & 151,500 & 33.8 & $213,9 \mathrm{C} 0$ \\
\hline 5.1 & 27,620 & 14.7 & 89,750 & 24.3 & 152,150 & 33.9 & 214,550 \\
\hline 5.2 & 28,240 & 14.8 & 90,400 & 24.4 & 152,800 & 34.0 & 215,200 \\
\hline 5.3 & 28,860 & 14.9 & 91,050 & 24.5 & 153,450 & 34.1 & 215,850 \\
\hline 5.4 & 29,480 & 15.0 & 91,700 & 24.6 & 154,100 & 34.2 & 216,500 \\
\hline 5.5 & 30,100 & 15.1 & 92,350 & 24.7 & 154,750 & 34.3 & 217.150 \\
\hline 5.6 & 30,720 & 15.2 & 93,000 & 24.8 & 155,400 & 34.4 & 217.800 \\
\hline 5.7 & 31,340 & 15.3 & 93,650 & 24.9 & 156,050 & 34.5 & 218.450 \\
\hline 5.8 & 31,960 & 15.4 & 94,300 & 25.0 & 156,700 & 34.6 & 219,100 \\
\hline 5.9 & 32,580 & 15.5 & 94,950 & 25.1 & 157,350 & 34.7 & 219,750 \\
\hline 6.0 & 33,200 & 15.6 & 95,600 & 25.2 & 158,000 & 34.8 & $220.4 \subset 0$ \\
\hline 6.1 & 33,850 & 15.7 & 96,250 & 25.3 & 158,650 & 34.9 & 221.050 \\
\hline 6.2 & 34,500 & 15.8 & $96.900 \mid$ & 25.4 & 159,300 & 35.0 & 221,700 \\
\hline 6.3 & 35,150 & 15.9 & 97,550 & 25.5 & 159,950 & 35.1 & 222350 \\
\hline 6.4 & 35,800 & 16.0 & 98,200 & 25.6 & 160,600 & 35.2 & 223,000 \\
\hline 6.5 & 36,450 & 16.1 & 98,850 & 25.7 & 161,250 & 35.3 & 223.650 \\
\hline 6.6 & 37,100 | & 16.2 & 99,500 & 25.8 & $161,900 \mid$ & 35.4 & 224,300 \\
\hline 6.7 & 37,750 & 16.3 & 100,150 & 25.9 & 162,550 & 35.5 & 224.950 \\
\hline
\end{tabular}


Rating table for Tennessee River at Chattanooga, Tenn., for 1901.

\begin{tabular}{|c|c|c|c|c|c|c|c|}
\hline 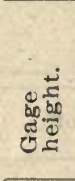 & 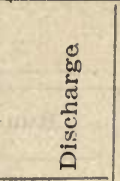 & 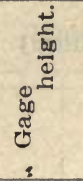 & 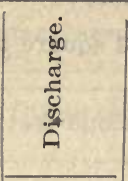 & 点 & 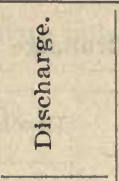 & & 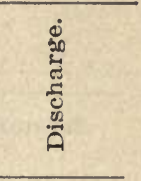 \\
\hline Feet. & Second $F t$. & Feet. & Secund $F t$. & Feet. & Second $f t$. & Feel. & Second $F t$. \\
\hline 6.8 & 38,400 & 16.4 & 100,800 & 26.0 & 163,200 & 35.6 & 225,600 \\
\hline 6.9 & 39,050 & 16.5 & 101,450 & 26.1 & 163,850 & 35.7 & 226,250 \\
\hline 7.0 & 39,700 & 16.6 & 102,100 & 26.2 & 164,500 & 35.8 & 226,900 \\
\hline 7.1 & 40,350 & 16.7 & 102,750 & 26.3 & 165,150 & 35.9 & 227,550 \\
\hline 7.2 & 41,000 & 16.8 & 103,400 & 26.4 & 165,800 & 36.0 & 228,200 \\
\hline 7.3 & 41,650 & 16.9 & 104,050 & 26.5 & 166,450 & 36.1 & 228,850 \\
\hline 7.4 & 42,300 & 17.0 & 104,700 & 26.6 & 167,100 & 36.2 & 229,500 \\
\hline 7.5 & 42,950 & 17.1 & 105,350 & 26.7 & 167,750 & 36.3 & 230,150 \\
\hline 7.6 & 43,600 & 17.2 & 106,000 & 26.8 & 168,400 & 36.4 & 230,800 \\
\hline 7.7 & 44,250 & 17.3 & 106,650 & 26.9 & 169,050 & 36.5 & 231,450 \\
\hline 7.8 & 44,900 & 17.4 & 107,300 & 27.0 & 169,700 & 36.6 & 232,100 \\
\hline 7.9 & 45,550 & 17.5 & 107,950 & 27.1 & 170,350 & 36.7 & 232,750 \\
\hline 8.0 & 46,200 & 17.6 & 108,600 & 27.2 & 171,000 & 36.8 & 233,400 \\
\hline 8.1 & 46,850 & 17.7 & 109,250 & 27.3 & 171,650 & 36.9 & 234,050 \\
\hline 8.2 & 47,500 & 17.8 & 109,900 & 27.4 & 172,300 & 37.0 & 234,700 \\
\hline 8.3 & 48,150 & 17.9 & 110,550 & 27.5 & 172,950 & 37.1 & 235,350 \\
\hline 8.4 & 48,800 & 18.0 & 111.200 & 27.6 & 173,600 & 37.2 & 233,000 \\
\hline 8.5 & 49,450 & 18.1 & 111,850 & 27.7 & 174,250 & 37.3 & 236,650 \\
\hline 8.6 & 50,100 & 18.2 & 112,500 & 27.8 & 174,900 & 37.4 & 237,300 \\
\hline 8.7 & 50,750 & 18.3 & 113,150 & 27.9 & 175,550 & 37.5 & 237,950 \\
\hline 8.8 & 51,400 & 18.4 & 113,800 & 28.0 & 176,200 & 37.6 & 238,600 \\
\hline 8.9 & 52,050 & 18.5 & 114,450 & 28.1 & 176,850 & 37.7 & 239,250 \\
\hline 9.0 & 52,700 & 18.6 & 115,100 & 28.2 & 177,500 & 37.8 & 239,900 \\
\hline 9.1 & 53,350 & 18.7 & 115,750 & 28.3 & 178,150 & 37.9 & 240,550 \\
\hline 9.2 & 54,000 & 18.8 & 116,400 & 28.4 & 178,800 & 38.0 & $2: 1,200$ \\
\hline 9.3 & 54,650 & 18.9 & 117,050 & 28.5 & 179,450 & 38.1 & 241,850 \\
\hline 9.4 & 55,300 & 19.0 & 117,700 & 28.6 & 180,100 & 38.2 & 242,500 \\
\hline 9.5 & 55,950 & 19.1 & 118,350 & 28.7 & $\cdot 180,750$ & 38.3 & 243,150 \\
\hline 9.6 & 56,600 & 19.2 & 119,000 & 28.8 & 181,400 & 38.4 & 243,800 \\
\hline 9.7 & 57,250 & 19.3 & 119,650 & 28.9 & 182,050 & 38.5 & 244,450 \\
\hline 9.8 & 57,900 & 19.4 & 120,300 & 29.0 & 182,700 & 38.6 & 245,100 \\
\hline 9.9 & 58,550 & 19.5 & 120,950 & 29.1 & 183,350 & 38.7 & 245,750 \\
\hline 10.0 & 59,200 & 19.6 & 121,600 & 29.2 & 184,000 & 38.8 & 246,400 \\
\hline 10.1 & 59,850 & 19.7 & 122,250 & 29.3 & 184,650 & 38.9 & 247,050 \\
\hline 10.2 & 60,50 & 19.8 & 122,900 & 29.4 & 185,300 & 39.0 & 247,700 \\
\hline 10.3 & 61,150 & 19.9 & 123,550 & 29.5 & 185,950 & 39.1 & 248,350 \\
\hline 10.4 & 61,800 & 20.0 & 124,200 & 29.6 & 186,600 & 39.2 & 249,000 \\
\hline 10.5 & 62,450 & 20.1 & 124,850 & 29.7 & 187,250 & 39.3 & 249,650 \\
\hline 10.6 & 63,100 & 20.2 & 125,500 & 29.8 & 187,900 & 39.4 & 250,300 \\
\hline 10.7 & 63,750 & 20.3 & 126,150 & 29.9 & 188,550 & 39.5 & 250,950 \\
\hline 10.8 & 64,400 & 20.4 & 126,800 & 30.0 & 189,200 & 39.6 & 251,600 \\
\hline 10.9 & 65,050 & 20.5 & 127,450 & 30.1 & 189,850 & 39.7 & 252,250 \\
\hline 11.0 & 65,700 & 20.6 & 128.100 & 30.2 & 190,500 & 39.8 & 252,900 \\
\hline 11.1 & 66,350 & 20.7 & 128,750 & 30.3 & 191,150 & 39.9 & $253,55 n$ \\
\hline 11.2 & 67,000 & 20.8 & 129,400 & 30.4 & 181,800 & 40.0 & 254,200 \\
\hline 11.3 & 67,650 & 20.9 & 130,050 & 30.5 & 192,450 & & \\
\hline 11.4 & 68,300 & 21.0 & 130,700 & 30.6 & 193,100 & & \\
\hline 11.5 & 68,950 & 21.1 & 131,350 & 30.7 & 193,750 & & \\
\hline
\end{tabular}

NoTE-This table applied to the foregoing "daily gage heights" gives the cubic feet per second flowing in the river on each date for which the gage height is given. 
Estimated monthly discharge of Tennessee River at Chattanooga, Tennessee.

[Drainage area, 21,382 square miles.]

\begin{tabular}{|c|c|c|c|c|c|c|c|}
\hline \multirow[b]{2}{*}{ Month. } & \multirow[t]{2}{*}{ • } & \multicolumn{3}{|c|}{ Discharge in second-feet } & \multirow[b]{2}{*}{$\begin{array}{l}\text { Total in } \\
\text { acre- } \\
\text { feet. }\end{array}$} & \multicolumn{2}{|c|}{ Run-off. } \\
\hline & & $\begin{array}{l}\text { Maxi- } \\
\text { mum. }\end{array}$ & $\begin{array}{l}\text { Mini- } \\
\text { mum. }\end{array}$ & Mean. & & $\begin{array}{l}\text { Depth } \\
\text { in } \\
\text { inches. }\end{array}$ & $\begin{array}{l}\text { Second. } \\
\text { feet per } \\
\text { square } \\
\text { mile. }\end{array}$ \\
\hline 1890. & & & & & & & \\
\hline January & & 78,470 & 30,150 & 42,749 & $2,628,551$ & 2.31 & 2.00 \\
\hline February & $\ldots$ & 308,060 & 44,191 & 76,081 & $4,225,310$ & 3.72 & 3.56 \\
\hline March ... & . $\quad \ldots \ldots \ldots$ & 445,120 & 52,906 & 129,093 & $7,937,670$ & 6.96 & 6.03 \\
\hline April .... & $\ldots \ldots \ldots$ & 121,464 & 43,029 & 64,855 & $3,859,132$ & 3.38 & 3.03 \\
\hline May ..... & ...... & 72,079 & 41,286 & 51,200 & $3,148,186$ & 2.76 & 2.39 \\
\hline June .... & $\ldots \ldots \ldots$ & 41,286 & 23,400 & 29,102 & $1,731,685$ & 1.52 & 1.36 \\
\hline July $\ldots .$. & . $\quad \ldots \ldots \ldots$ & 47,677 & 19,500 & 27,036 & $1,662,390$ & 1.45 & 1.26 \\
\hline August ... & $\ldots \ldots \ldots$ & 46,515 & 21,100 & 30,881 & $1,898,810$ & 1.66 & 1.44 \\
\hline September & $\ldots \ldots$ & 47,096 & 22,200 & 27,843 & $1,656,770$ & 1.45 & 1.30 \\
\hline October .. & $\ldots \ldots \ldots$ & 58,135 & 25,950 & 37,982 & $2,335,437$ & 2.04 & 1.77 \\
\hline November & $\ldots \ldots$ & 42,448 & 20,700 & 26,394 & $1,570,549$ & 1.37 & 1.23 \\
\hline December & $\ldots \ldots$ & $\begin{array}{r}77,889 \\
\end{array}$ & 20,400 & 36,088 & $2,218,980$ & 1.95 & 1.69 \\
\hline Per ann & um .... & 445,120 & 19,500 & 48,275 & $34,873,470$ & 30.57 & 2.26 \\
\hline 1891. & & & & & & & \\
\hline January & $\ldots$ & 92,995 & 39,543 & 59,484 & $3,657,552$ & 3.21 & 2.78 \\
\hline February & $\ldots$ & 356,120 & 59,878 & 154,822 & $8,598,380$ & 7.53 & 7.23 \\
\hline March ... & 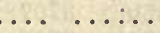 & 381,040 & 63,364 & 135,160 & $8,310,718$ & 7.30 & 6.32 \\
\hline April .... & $\ldots \ldots \ldots$ & 97,643 & 38,962 & 61,873 & $3,681,690$ & 3.22 & 2.89 \\
\hline May ..... & . $\quad \ldots \ldots \ldots$ & 37,219 & 26,380 & 30,215 & $1,857,860$ & 1.63 & 1.41 \\
\hline June & & 47,096 & 26,840 & 36,276 & $2,158,567$ & 1.90 & 1.70 \\
\hline July .. & . . . & 36,057 & 21,800 & 26,429 & $1,625,066$ & 1.43 & 1.24 \\
\hline August .. & .. . & 98,224 & 23,000 & 40,402 & $2,484,238$ & 2.18 & 1.89 \\
\hline September & $\ldots$ & 36.638 & 19,200 & 25,777 & $1,533,835$ & 1.34 & 1.20 \\
\hline October .. & $\ldots \quad \ldots$ & 19,200 & 17,910 & 18,461 & $1,135,130$ & .99 & .86 \\
\hline November & $\ldots$ & 41,867 & 17,440 & 23,510 & $1,398,939$ & 1.23 & 1.10 \\
\hline December & $\ldots \ldots$ & 66,269 & 22.200 & 39,299 & $2,416,417$ & 2.12 & 1.84 \\
\hline Per ann & $\mathrm{um} \ldots$ & 381,040 & 17,440 & 54,309 & $38,858,392$ & 34.08 & 2.54 \\
\hline & & & & & & & \\
\hline January & & 363,240 & 41,286 & 103,453 & $6,361,118$ & 5.57 & 4.83 \\
\hline February & & 69,755 & 33,733 & 46,755 & $2,689,348$ & 2.43 & 2.25 \\
\hline March .. & & 64,526 & 31,680 & \begin{tabular}{|l|l|} 
& 45,769
\end{tabular} & $2,814,244$ & 2.47 & 2.14 \\
\hline April & & 299,160 & 40,705 & 101,287 & $6,026,982$ & 5.27 & 4.73 \\
\hline May .. & & 53,487 & 31,160 & 39,772 & $2,445,501$ & 2.14 & 1.86 \\
\hline June . & & 56,972 & 28.680 & 43,265 & $2,574,447$ & 2.25 & 2.02 \\
\hline July & & 71,498 & 26,380 & 44,520 & $2,737,446$ & 2.40 & 2.08 \\
\hline August ... & & 31,680 & 21,800 & 25,121 & $1,544,640$ & 1.35 & 1.17 \\
\hline September & - $\ldots$ & 29,660 & 17,660 & 21,403 & $1,273,564$ & 1.11 & 1.00 \\
\hline October . & & 19,800 & 17,220 & 17,952 & $1,103,833$ & .97 & .84 \\
\hline November & $\ldots \ldots \ldots$ & 43,610 & 17,220 & 27,924 & $1,661,590$ & 1.45 & 1.30 \\
\hline December & $\ldots$ & 56,972 & 21,450 & 32,793 & $2,016,376$ & 1.76 & 1.53 \\
\hline Per ann & aum $\ldots \ldots$ & $|363,240|$ & 17,220 & 45,835 & $33,249,089$ & .29 .17 & 2.15 \\
\hline
\end{tabular}


Estimated monthly discharge of Tennessee River at Chattanooga, T'ennessee-Continued.

[Drainage area, 21,382 square miles.]

\begin{tabular}{|c|c|c|c|c|c|c|}
\hline \multirow[b]{2}{*}{ Month. } & \multicolumn{3}{|c|}{$\begin{array}{l}\text { Discharge in second- } \\
\text { feet. }\end{array}$} & \multirow[b]{2}{*}{$\begin{array}{l}\text { Total in } \\
\text { acre-ft. }\end{array}$} & \multicolumn{2}{|c|}{ Run-off. } \\
\hline & 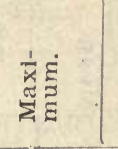 & $\underset{Z}{\frac{1}{\Xi}}$ & $\frac{\dot{E}}{\frac{E}{2}}$ & & 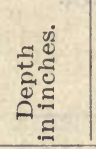 & 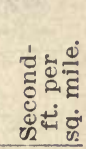 \\
\hline $\begin{array}{l}1893 . \\
\text { January }\end{array}$ & & 22,600 & 26,812 & $1,648,616$ & 1.44 & 1.25 \\
\hline $\begin{array}{l}\text { January } \\
\text { February }\end{array}$ & $\left|\begin{array}{r}44,191 \\
283,140\end{array}\right|$ & 38,381 & 105,921 & $\begin{array}{l}1,040,010 \\
5,882,535\end{array}$ & $\begin{array}{l}1.44 \\
5.15\end{array}$ & $\begin{array}{l}1.20 \\
4.95\end{array}$ \\
\hline March ........ & 72,660 & 36,057 & 50,320 & $3,094,076$ & 2.71 & 2.35 \\
\hline April ......... & 73,241 & 31,160 & 42,137 & $2,507,326$ & 9.20 & 1.97 \\
\hline May $\ldots \ldots \ldots \ldots$ & 224,400 & 30,150 & 71,525 & $4,397,929 !$ & 3.85 & 3.34 \\
\hline June $. . . \ldots \quad \ldots . .$. & 123,207 & 27,760 & 49,679 & $2,956,099 i$ & $2.59 !$ & 2.32 \\
\hline July $\ldots$ & 34,895 & 21,450 & 25,741 & $1,582,763$ & $1.39 !$ & 1.20 \\
\hline ugust $\ldots \ldots$.... & 33,152 & 18,410 & 23,477 & $1,443,554$ & 1.27 & 1.10 \\
\hline eptember ... & 76,7 & 20,7 & 33,933 & 2,0 & 1.77 & 1.59 \\
\hline ctober ... & 58,716 & 18,660 & 25,550 & 1,57 & 1.37 & 1.19 \\
\hline November $\ldots . . .$. & 31 , & 20 & 22,2 & & 1. & 1.04 \\
\hline December ........ & 30,640 & 21,100 & 24,970 & $1,535,355$ & 1.35 & 1.17 \\
\hline Per annum $\ldots . .$. & 283,140 & 18,410 & 41,861 & $29,963,158$ & 26.24 & 1.96 \\
\hline & & & & & & \\
\hline January . & 56,972 & 22,600 & 37,389 & $2,298,975$ & 2.02 & 1.75 \\
\hline February $\ldots . . .$. & 151,095 & 31,6 & 0,893 & 3,9 & 3.45 & 3.81 \\
\hline $\operatorname{March} \ldots \ldots \ldots$ & 59,297 & 33,152 & 46,796 & 7,392 & 2.53 & 2.19 \\
\hline April $\ldots \ldots \ldots \ldots$ & 52,325 & 27,300 & 36,287 & $2,159,222$ & 1.90 & 1.79 \\
\hline May ........ & 44,191 & 23,800 & 31,137 & $1,914,552$ & 1.68 & 1.43 \\
\hline June $\ldots \ldots \ldots$..... & 28, & 19,500 & 21,983 & $1,308,076$ & 1.15 & 1.03 \\
\hline July $\ldots \ldots \ldots$. $\ldots \ldots$ & 29,1 & 18,910 & 24,486 & $1,505,595$ & 1.31 & 1.14 \\
\hline August $\ldots \ldots \ldots \ldots$ & & 3,910 & 22,971 & 2,441 & 1.23 & 1.07 \\
\hline September $\ldots . . .$. & 27 , & 16 & 19,1 & 1,1 & 1.00 & .90 \\
\hline October $\ldots \ldots \ldots$..... & 20,750 & 16,360 & & & .94 & .82 \\
\hline November $\ldots . . .$. & 20,4 & 16,360 & & 1,0 & .90 & .81 \\
\hline December ........ & 68,012 & 16,780 & & $1.897,643$ & 1.66 & 1.44 \\
\hline Per annum $\ldots . .$. & 151,095 & 16,360 & 31,395 & $|22,555,040|$ & 19.77 & 1.53 \\
\hline & & & & & & \\
\hline $\begin{array}{l}\text { January } \\
\text { February }\end{array}$ & 47,0 & $\begin{array}{l}23,4 \\
24,2\end{array}$ & 35, & & $\begin{array}{l}4.12 \\
1.74\end{array}$ & 1.67 \\
\hline March $\ldots \ldots \ldots$ & 134,827 & 42,448 & 72,341 & & 3.90 & 3.38 \\
\hline April $\ldots \ldots \ldots \ldots$ & & 37,219 & & & 2.67 & 2.39 \\
\hline May $\ldots \ldots \ldots \ldots$ & & 34,314 & 43,929 & 2,7 & 2.37 & 2.05 \\
\hline June $\ldots \ldots$. $\ldots \ldots$. & 35,476 & 21,100 & $\angle 6,417$ & $1,651,917$ & 1.37 & 1.23 \\
\hline July $\ldots \ldots \ldots \ldots$ & & & & & 1.60 & 1.39 \\
\hline August $\ldots \ldots \ldots \ldots$ & & 21,800 & 26,927 & $1,655,687$ & 1.45 & 1.26 \\
\hline September $\ldots . . .$. & 24,660 & 16,780 & 20,316 & $1,208,883$ & 1.05 & .95 \\
\hline October $\ldots \ldots \ldots$ & & & & & .90 & .78 \\
\hline November $\ldots . . .$. & 20,750 & 17,220 & 18,162 & $1,080,714$ & .94 & .85 \\
\hline December $\ldots \ldots \ldots$ & 33,152 & 17,660 & $21,561 \mid$ & $1,325,743$ & 1.16 & 1.01 \\
\hline Per annum ....... & & 16,360 & 36,603 & $26,564,748 \mid$ & 23.27 & 1.71 \\
\hline
\end{tabular}


Estimated monthly discharge of Tennessee River at Chattanooga, Tennessee-Continued.

[Drainage area, 21,418 square miles.]

\begin{tabular}{|c|c|c|c|c|c|c|}
\hline \multirow[b]{2}{*}{ Month. } & \multicolumn{3}{|c|}{$\begin{array}{l}\text { Discharge in second. } \\
\text { feet. }\end{array}$} & \multirow[b]{2}{*}{$\begin{array}{l}\text { Total in } \\
\text { acre- } \\
\text { feet. }\end{array}$} & \multicolumn{2}{|c|}{ Run-off. } \\
\hline & 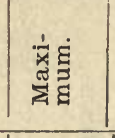 & 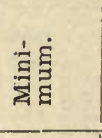 & $\frac{E}{\tilde{\sigma}}$ & & 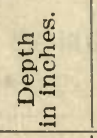 & 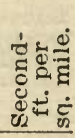 \\
\hline 1896. & & & & & & \\
\hline January ... ..... & 50,600 & 15,554 & 26,169 & $1,609,079$ & 1.41 & 1.22 \\
\hline February $\ldots \ldots \ldots$ & 85,052 & 26,840 & 55,577 & $3,196,826$ & 2.79 & 2.59 \\
\hline March $\ldots \ldots \ldots$ & 95,150 & 22,088 & 39,257 & $2,413,834$ & 2.11 & 1.83 \\
\hline April $\ldots \ldots \ldots \ldots$ & 409,520 & 23,276 & 87,649 & $5,215,478$ & 4.56 & 4.09 \\
\hline May $\ldots \ldots \ldots \ldots$ & 29,216 & 14,366 & 20,574 & $1,265,054$ & 1.10 & 0.96 \\
\hline June $\ldots \ldots . . . \ldots$ & 43,472 & 17,336 & 24,365 & $1,449,815$ & 1.27 & 1.14 \\
\hline July $\ldots \ldots \ldots \ldots \ldots$ & 130,196 & 20,306 & 55,390 & $3,405,820$ & 2.99 & 2.59 \\
\hline August $\ldots \ldots \ldots$ & 34,562 & 14,960 & 22,433 & $1,379,360$ & 1.21 & 1.05 \\
\hline September $\ldots \ldots$ & 18,524 & 9,020 & 12,346 & 734,636 & .64 & .58 \\
\hline October $\ldots \ldots \ldots$ & 19,712 & 8,426 & 11,588 & 712,523 & .62 & .54 \\
\hline November $\ldots \ldots \ldots$ & 57,728 & 9,020 & 22,603 & $1,344,969$ & 1.18 & 1.06 \\
\hline December ........ & 45,254 & 16,148 & 27,951 & $1,718,651$ & 1.51 & 1.31 \\
\hline The year...... & 409,520 & 8,426 & 33,825 & $\mid 24,446,045$ & 21.39 & 1.58 \\
\hline 1897. & & & & & & \\
\hline January .... & 45,254 & 16,148 & 27,932 & $1,717,483$ & 1.50 & 1.30 \\
\hline February $\ldots \ldots \ldots$ & 308,060 & 19,712 & 89,962 & $4,996,236$ & 4.37 & 4.20 \\
\hline March $\ldots \ldots \ldots$ & 363,240 & 52,976 & 165,448 & $10,173,067$ & 8.90 & 7.72 \\
\hline April $\ldots \ldots \ldots \ldots$ & 231,520 & 36,344 & 81,056 & $4,823,156$ & 4.22 & 3.78 \\
\hline May $\ldots \ldots . \ldots \ldots$ & 134,948 & 26,543 & 50,124 & $3,082,025$ & 2.70 & 2.34 \\
\hline June $\ldots \ldots . . . .$. & 38,126 & 21,494 & 29,107 & $1,731,983$ & 1.52 & 1.36 \\
\hline July $\ldots \ldots \ldots \ldots$ & 74,657 & 21,791 & 34,428 & $2,116,909$ & 1.86 & 1.61 \\
\hline August $\ldots \ldots \ldots \ldots$ & 34,562 & 14,366 & 25,847 & $1,589,280$ & 1.39 & 1.21 \\
\hline September $\ldots . . .$. & 14,960 & 6,050 & 8,951 & 532,620 & .47 & .42 \\
\hline October $\ldots \ldots \ldots$ & 13,772 & 4,268 & 7,842 & 482,189 & .43 & .37 \\
\hline November $\ldots \ldots \ldots$ & 9,614 & 6,050 & $7,3 \doteq 0$ & 436,164 & .38 & .34 \\
\hline December $\ldots . . .$. & 62,183 & 8,129 & 24,627 & $1,514,265$ & 1.33 & 1.15 \\
\hline The year $\ldots . . .$. & $|363,240|$ & 4,268 & 46,055 & $\mid 33,195,377$ & 29.07 & 2.15 \\
\hline & & & & & & \\
\hline January ........... & 124,860 & 14,344 & 59,509 & $3,778,785$ & 3.20 & 2.77 \\
\hline February $\ldots \ldots \ldots \ldots$ & 47,115 & 16,900 & 22,994 & $1,277,022$ & $1.11 \mid$ & 1.07 \\
\hline March $\ldots \ldots \ldots$ & 88,725 & 15,008 & 24,774 & $1,523,304$ & 1.28 & 1.11 \\
\hline April $\ldots \ldots \ldots \ldots$ & $|121,940|$ & 39,085 & 60,048 & $3,573,096$ & 3.12 & 2.80 \\
\hline May $\ldots \ldots \ldots \ldots$ & 38,720 & 17,920 & 23,701 & $1,457,327$ & 1.28 & 1.11 \\
\hline June ...... & 32,515 & 10,245 & 16,395 & 975,569 & .85 & .77 \\
\hline July ...... & 49,670 & 11,853 & 20,063 & $1,233,633$ & 1.08 & .94 \\
\hline August $\ldots \ldots \ldots$ & 107,705 & 20,410 & 50,638 & $3,113,629$ & 2.72 & 2.36 \\
\hline September $\ldots \ldots \ldots$ & 174,500 & 18,190 & 47,349 & $2,817,454$ & 2.46 & 2.21 \\
\hline October $\ldots . . . . .$. & $\mid 115,370$ & 16,900 & 44,215 & $2,718,691$ & 2.38 & 2.06 \\
\hline November $\ldots . . .$. & 42,005 & 20,700 & 28,415 & $1,690,806$ & 1.48 & 1.33 \\
\hline December $\ldots . . . .$. & 35,800 & 19,000 & 28,909 & $1,777,556$ & 1.56 & 1.25 \\
\hline The year $\ldots . . .$. & $|174,500|$ & 10,245 & 35,584 & $\mid 25,936,872$ & 22.52 & 1.66 \\
\hline
\end{tabular}


Estimated monthly discharge of Tennessee River at Chattanooga, Tennessee-Continued.

[Drainage area, 21,418 square miles.]

\begin{tabular}{|c|c|c|c|c|c|c|}
\hline \multirow[b]{2}{*}{ Month. } & \multicolumn{3}{|c|}{$\begin{array}{l}\text { Discharge in } \\
\text { second-feet. }\end{array}$} & \multirow{2}{*}{$\begin{array}{l}\text { Total in } \\
\text { acre- } \\
\text { feet. }\end{array}$} & \multicolumn{2}{|c|}{ Run-off. } \\
\hline & $\begin{array}{l}\text { Maxi- } \\
\text { mum. }\end{array}$ & $\begin{array}{l}\text { Mini- } \\
\text { mum. }\end{array}$ & Mean. & & $\begin{array}{c}\text { Depth } \\
\text { in } \\
\text { inches. }\end{array}$ & $\begin{array}{c}\text { feet per } \\
\text { square } \\
\text { mile. }\end{array}$ \\
\hline 1899. & & & & & & \\
\hline January ... & 112,560 & 25,450 & 47,250 & $2,905,289$ & 2.55 & 2.21 \\
\hline February .... & 233,150 & 30,720 & 95,554 & $5,306,801$ & 4.64 & 4.46 \\
\hline March $\ldots \ldots \ldots \ldots$ & 244,000 & 55,210 & 142,700 & $8,774,281$ & 7.68 & 6.66 \\
\hline April $\ldots \ldots \ldots \ldots$ & 137,360 & 39,710 & 69,286 & $4,122,803$ & 3.59 & 3.23 \\
\hline May $\ldots \ldots \ldots$. & 65,130 & 22,040 & 40,450 & $2.487,173$ & 2.18 & 1.89 \\
\hline June $\ldots . . .$. & 35,990 & 15,600 & 23,088 & $1,373,831$ & 1.20 & 1.08 \\
\hline July $\ldots \ldots \ldots$ & 27,930 & 10,430 & 15,053 & 925,573 & .81 & .70 \\
\hline August $\ldots .$. & 22,040 & 8,040 & 11.900 & 731,702 & .64 & .56 \\
\hline September ... & 15,840 & 7,300 & 10,118 & 603,063 & .53 & .47 \\
\hline October $\ldots \ldots \ldots$ & 10,635 & 6,600 & 7,851 & 482,739 & .43 & .37 \\
\hline November .......... & 10,635 & 6,775 & 8,216 & 488,886 & .43 & .38 \\
\hline December $\ldots . . . .$. & 41,880 & 8,820 & 22,061 & $1,356,478$ & 1.19 & 1.03 \\
\hline i'he year .. & $|244,000|$ & 6,600 & 41,127 & $29,557,619$ & 25.87 & 1.09 \\
\hline 1900. & & & & & & \\
\hline January & 54,280 & 11,660 & 30,807 & $1,894,248$ & 1.66 & 1.44 \\
\hline February .... & 144,800 & 13.360 & 52,077 & $2,892,210$ & 2.53 & 2.43 \\
\hline March ....... & 103,880 & 42,810 & 66,020 & $4,059,412$ & 3.55 & 3.08 \\
\hline April $\ldots \ldots \ldots$ & 70,400 & 32,820 & 46,819 & 5,924 & 2.44 & 2.19 \\
\hline May $\ldots \ldots \ldots \ldots$ & 34,440 & 15,600 & 21,086 & $1,296,528$ & 1.13 & .98 \\
\hline June ....... & 53,040 & 14,680 & 33,295 & $1,981,190$ & 1.73 & 1.55 \\
\hline July ........ & 50,870 & 13,360 & 24,674 & $1,517,145$ & 1.33 & 1.15 \\
\hline August $\ldots \ldots$. & 34,440 & 10,020 & 14,602 & 897,841 & .78 & .68 \\
\hline September ... & 25,140 & 7,300 & 13,393 & 796,939 & .70 & .63 \\
\hline October ...... & 42,500 & 8,040 & 14,230 & 874,968 & .76 & .66 \\
\hline November .... & 92,720 & 10,840 & 25,138 & $1,495,815$ & $1.31\}$ & 1.17 \\
\hline December .. & 53,040 & 17,050 & 29,001 & $1,783,201$ & $1.56 !$ & 1.35 \\
\hline The year ........ & $|144,800|$ & 7,300 & 30,928 & $22.275,421 \mid$ & $19.48 \mid$ & 1.44 \\
\hline & & & & & & \\
\hline January . & & 189,2 & 19,6 & 50,641 & 2.72 & 2.36 \\
\hline February. & & . 59,8 & 19,0 & 36,516 & $1.77 !$ & 1.70 \\
\hline March $\ldots \ldots \ldots \ldots$ & & . $\mid 139,1$ & 19,0 & 44,952 & 2.42 & 2.10 \\
\hline April .. & & 166,4 & 38,4 & 95.080 & 4.95 & 4.44 \\
\hline May $\ldots \ldots \ldots \ldots \ldots$ & ......... & $\ldots 205,4$ & 26,3 & 68.736 & 3.701 & 3.21 \\
\hline June $\ldots \ldots \ldots \ldots \ldots$ & ....... & .. 72,2 & $00 \mid 33,8$ & 47,673 & 2.49 & 2.23 \\
\hline July ........... & . $\ldots$. & .137 .1 & $00 \mid 14,6$ & 23.932 & 1.29 & 1.12 \\
\hline August...$\ldots \ldots \ldots$ & ....... & $\ldots 1207,4$ & $00 \mid 13.8$ & 75,761 & 4.081 & 3.54 \\
\hline September ......... & & . 61.1 & $50 \mid 22,6$ & 38.859 & 2.021 & 1.81 \\
\hline October $\ldots \ldots \ldots$ & & 25.7 & 60। 13,3 & $60 \mid 18.979$ & $1.03 !$ & .89 \\
\hline November ........ & & 13,8 & $00 \mid 11,6$ & $60 \mid 13,076$ & .681 & .61 \\
\hline December ......... & & 237.3 & $00 \mid 12,0$ & 65.509 & 3.531 & 3.06 \\
\hline The year $\ldots . . \ldots$ & & $\mid 237,2$ & $00 \mid 11,6$ & 48,310 & 30.68 & 2.26 \\
\hline
\end{tabular}




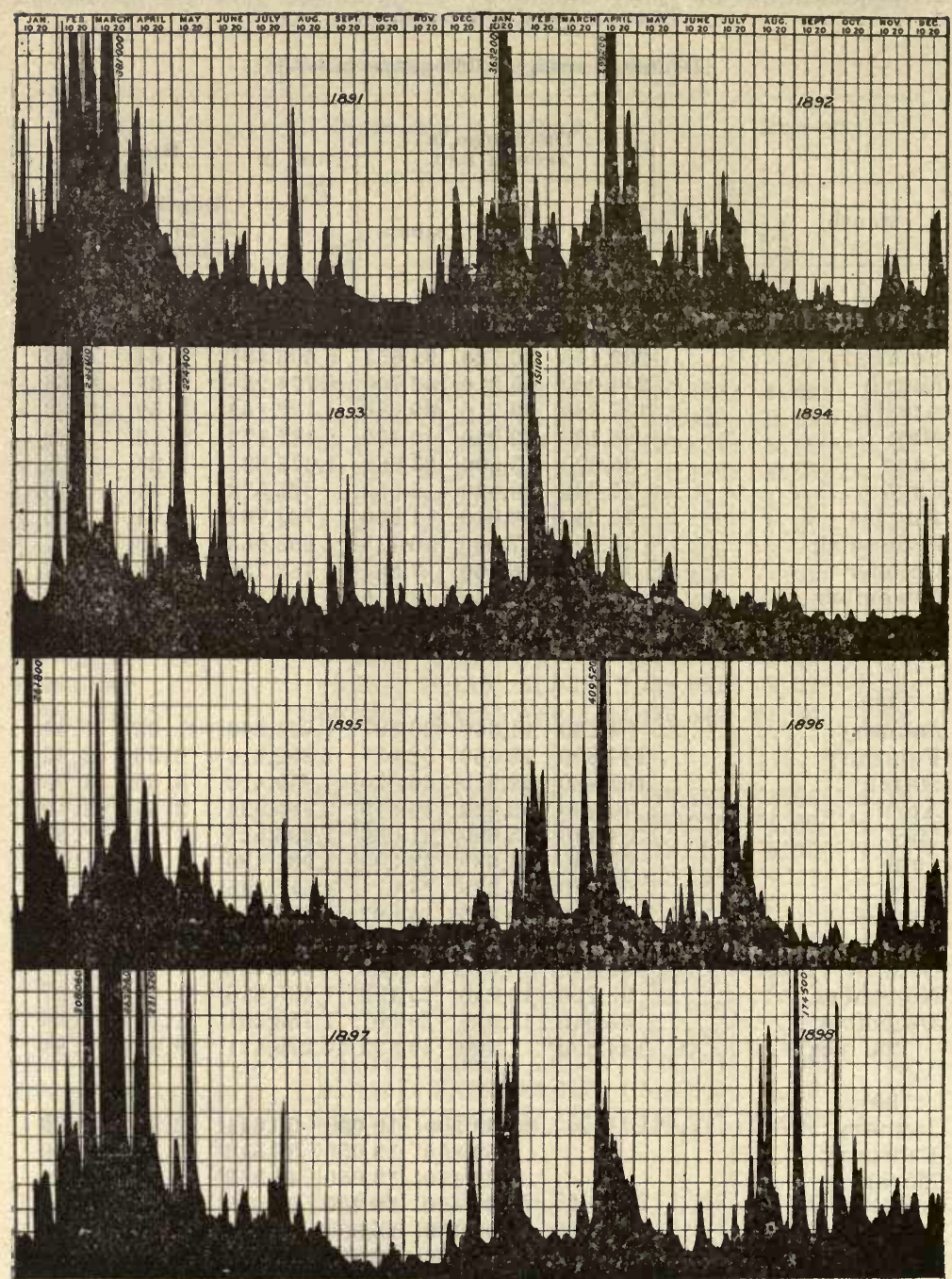

Fig. 16-Discharge of Tennessee River at Chattanooga, Tenn, 1891-1898. 
Minimum monthly discharge of Tennessee River at Chattanooga, Tenn., with corresponding net horsepower per foot of fall on $a$ water wheel realizing 80 per cent. of the theoretical power.

\begin{tabular}{|c|c|c|c|c|c|c|c|c|c|}
\hline & & 1899. & & & 1300. & & & 1961. & \\
\hline & 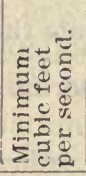 & 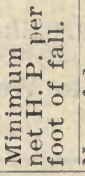 & & 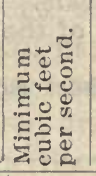 & 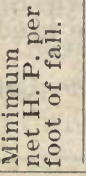 & & 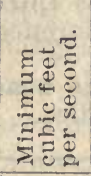 & 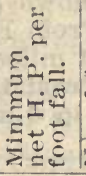 & 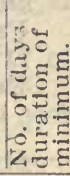 \\
\hline & 5,450 & 2,314 & 1 & 11,660 & 1,060 & 1 & 19,600 & 1.782 & \\
\hline ebruary & 30,720 & 2,793 & 1. & 13,360 & 1,215 & 1 & 19,050 & 1,732 & \\
\hline $\operatorname{arch} . . .$. & 55,210 & 5,019 & 1 & 42,810 & 3,892 & 2 & 19,050 & 1,732 & \\
\hline -il & 39,71 & 3,610 & 1 & 32,820 & 3,075 & 1 & 38,400 & 3.491 & \\
\hline & 22,040 & 2,004 & 1 & 15,600 & 1,418 & 1 & $23,3 \leq 0$ & 2,398 & \\
\hline une..... & 15,600 & 1,418 & 1 & 14,6 & 1,335 & 1 & 33,850 & 3.077 & \\
\hline $1 \pi \quad-25$ & $10,4 i$ & 948 & 1 & 13,3 & 1,215 & 2 & 14,680 & 1,335 & \\
\hline & 8,0 & & 3 & 10,0 & 911 & 1 & & 1,255 & \\
\hline mber .. & 7,30 & 664 & 1 & & 664 & 1 & 22.660 & 2,060 & ) \\
\hline October .... & 6,600 & 600 & 3 & 8,040 & 731 & 2 & 13,360 & 1,215 & 5 \\
\hline ber .. & & 616 & 2 & & 985 & 2 & 11,660 & 1,060 & ) \\
\hline December .. & & 802 & 2 & 17,050 & 1,550 & 2 & 12,080 & 1,098 & 8 \\
\hline
\end{tabular}

Note-To find the minimum net horse power available at a shoal on this stream, near this station, for any month, multiply the total fall of the shoal by the "net H. P. per foot of fall" in this table for that month.

2. SHOALS IN TENNESSEE RIVER NEAR FLORENCE, ALABAMA.

In Tennessee River, in the vicinity of Florence, Ala., (see Fig. 96), are several shoals capable of the development of power in large quantities. The compiler has brought together the data regarding these, his intention being not to discuss the manner in which the immense water power of these shoals can be developed, but to give some idea of its magnitude and the possibility of its utilization.

The shoals are a succession of cascades amid many islands, in a river bed varying in width from a half mile to three miles. The numerous channels thus formed are very irregular in fall and direction. The difference between high and low water is only 5 or 6 feet, corresponding to a rise of 50 feet at Chattanooga. Beginning at Brown's Ferry, 12 miles below Decatur. Ala., the river has the following falls: 
From Browns Ferry to the mouth of Elk River the fall is 26 feet in 11 miles. This is known as Elk River Shoals. Its most precipitous part is at the lower end, where there is a fall of 16.5 feet in about 4 miles.

From the mouth of Elk River to the head of Muscle Shoals, a distance of 5 miles, there is a fall of only 2 feet.

From the head of Muscle Shoals to Bainbridge the fall is 85 feet in 17 miles, and is known as Big Muscle Shoals.

From Bainbridge to Florence the fall is 23 feet in 7 miles, and is known as Little Muscle Shoals.

From Florence to the head of the Colbert Shoals the fall is 3 feet in 11 miles.

From the head of the Colbert Shoals to Waterloo the fall is 21 feet in 6 miles.

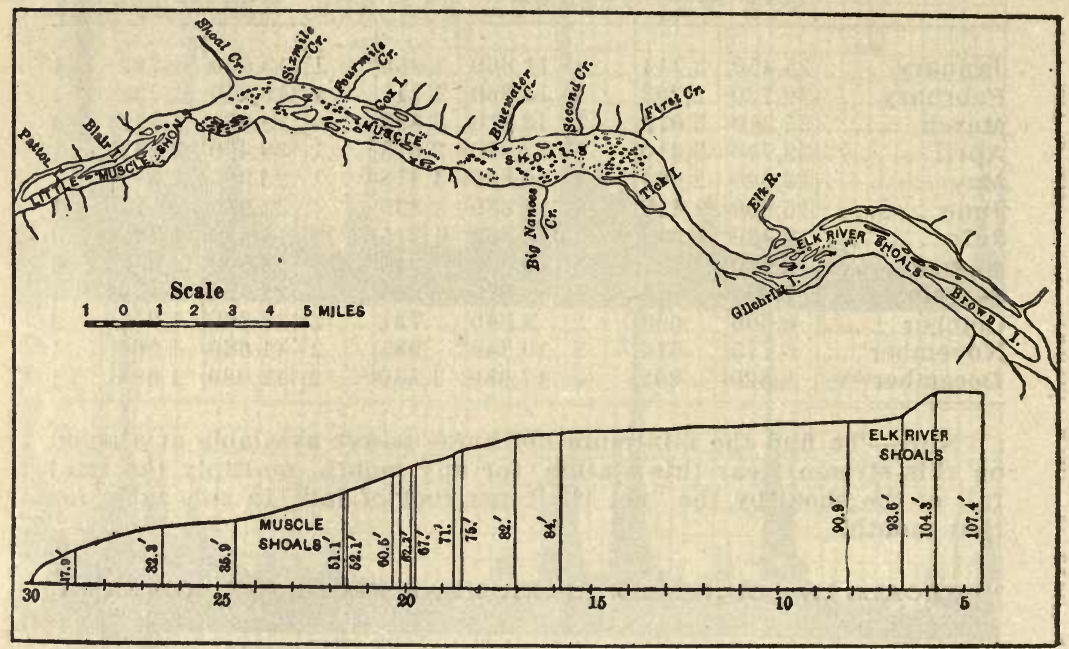

Fig. 17.-Map showing shoals in Tennessee River, near Florence, Ala.

The total fall from Browns Ferry to Waterloo is, therefore, I6o feet in a distance of 57 miles. Sixteen miles of the distance, howeer, has a fall of only 5 feet, leaving a fall of 155 feet in the $4 \mathrm{I}$ miles that cover the four shoals mentioned. The shoals are really more precipitous than the foregoing figures would indicate. For instance, 84.6 feet of the fall at Big Muscle Shoals is in a distance of 14 miles.

The bed rock at Elk River Shoals is Carboniferous limestone; that of Muscle Shoals is a hard silicous rock of dark color and flinty structure.

The following is a statement of the minimum discharge of Tennessee River at Chattanooga :

*The numbers 5 to 30 at bottom of cut represent miles. 
From 1890 to 1895 , inclusive $\ldots \ldots \ldots \ldots \ldots \ldots \ldots \ldots \ldots \ldots \ldots \ldots \ldots \ldots$ 16.

From 1896 to 1900 , inclusive...................6. 600

From January 1 to November 16,1901 , inclusive ........ 12,930

From this it is estimated that 6,600 second-feet is the minimum discharge for driest years, and that 12,930 second-feet is the minimum for average years. Assuming that tributaries entering the river below Chattanooga will safely supply all of the water needed for lockage, we can use these discharges in estimating the water power of these shoals, which are about 200 miles below Chattanooga, by river, and drain an area more than 7,000 square miles greater than the watershed above Chattanooga.

Estimated minimum net horsepower of Tennessee River in Alabama on turbines realizing 80 per cent. of the theoretical power.

\begin{tabular}{|c|c|c|c|}
\hline \multirow[t]{2}{*}{ Locali.y. } & Fall & $\begin{array}{l}\text { Minimum } \\
\text { not p'wer in } \\
\text { driest ycars. }\end{array}$ & $\begin{array}{l}\text { Minimum } \\
\text { net power in } \\
\text { average } \\
\text { years. }\end{array}$ \\
\hline & Fall. & Horsepower, & Horsepower \\
\hline Elk River Shoals ................ & 26 & 15,600 & 30,050 \\
\hline Big Muscle Shoals ............ & 85 & 51,000 & 99.875 \\
\hline Little Muscle Shoals $\ldots \ldots \ldots \ldots \ldots \ldots$ & 23 & 13,800 & 27,025 \\
\hline Colbert Shoals .................. & 21 & 12,600 & 24.675 \\
\hline Total $\ldots \ldots \ldots \ldots \ldots$ & 155 & 93,000 & 182,125 \\
\hline
\end{tabular}

The foregoing table assumes that the total fall can in each case be utilized. While this assumption is not correct, it stands as an offset to the assumption that the water supply available will be as low as the minimum discharge at Chattanooga, 200 miles above. The drainage area above Chattanooga is $2 \mathrm{I}, 4 \mathrm{I} 8$ square miles, while the drainage area above the shoals under consideration is about 29,000 square miles. It may therefore safely be assumed that the actual power available for development at the shoals is greater than that shown by the table.

The foregoing statements of fall and distance are from a report by Mr. William B. Gaw, chief assistant engineer, United States Army, 1868, and the map and profile are from drawings prepared under the direction of Lieut. Col. J. W. Barlow, United States Engineers, I890. 
3. TRIBUTARIES OF TENNESSEE RIVER.

Paint Rock Creek, Elk River, Shoal Creek, Flint Creek, Big Nance Creek, Town Creek, and Big Bear Creek are all large streams, and most of them have fine undeveloped water powers. But no surveys have been made of them, and no measurements of discharge so far. There are also many large bold springs in this basin, that are said to have a pure and unfailing water supply, but the Hydrographic Survey has not reached them. and no report can be made on them at this time. 



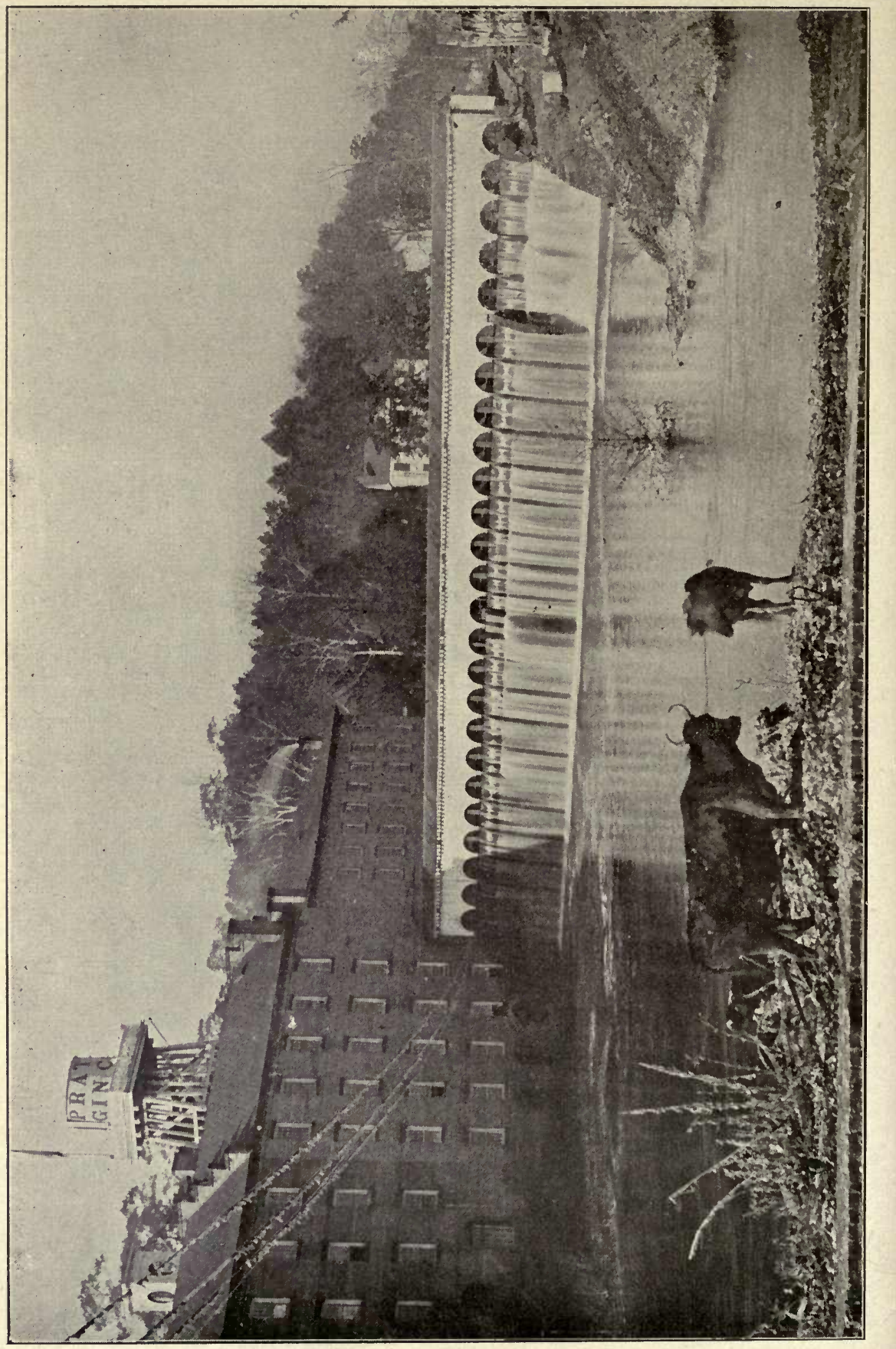




\section{CHAPTER VIII.}

\section{UTILIZED WATER POWERS OF ALABAMA.}

The following is a list by counties of the water powers that are utilized. The most of these powers are small, but they make a large aggregate, and they represent only an insignificant part of the power that is capable of development.

\section{*AUTAUGA COUNTY.}

NAME.

POSTOFFICE.

INDUSTRY.

H. P.

Charity P. Carter, Billingsley, flour and grist mill........ 15

Montgomery's Mill, Prattville, flour and grist mill ........ 30

Public Grist Mill, Billingsley, flour and grist mill ......... 9

Parker's Mill, Milton, flour and grist mill............. 20

Dawson's Mill, Netezen, lumber and timber mill.......... 20

Ellis Mill, Jones Switch, lumber and timber mill........... 4

Long Leaf Yellow Pine Saw Mill, Autaugaville, lumber and

Ray's Saw Mill, Jones Switch, lumber and timber mill....... 10

Swift Creek Mill vo. (Swift Creek), Autaugaville, lumber and timber mill ............................. 70

John H. Herod, Netezen, lumber and timber mill........... 6

Prattville Cotton M. \& Banking Co. (Autauga Creek), Prattville, cotton goods. The dam at Prattville is shown in

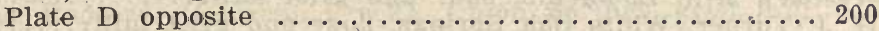

Continental Gin Co., (Autauga Creek), Prattville, cotton gin Prattville Ice Factory (Autauga Creek), Prattville, ice factory Doster Ginnery (Autauga Creek), Prattville, cotton gin..... G. H. Roy, Vine Hill, cotton gin $\ldots \ldots \ldots \ldots \ldots \ldots \ldots \ldots$

\section{WATER POWER AT PRATTVILLE.}

The water power at Prattville was first developed about I 830 , when it was used by a man named May to operate a small saw mill. About 1833 this water power and the adjacent lands were purchased by Mr. Daniel Pratt, who then erected a cotton gin factory, which was driven by the water power. The dam at that time was about eight feet high. A number of years after the purchase of this property by Mr. Pratt he increased the dam so that it now has a height of 16 feet, and is built of brick. At present it is used jointly by the Prattville Cotton Mills \& Banking Company and the Continental Gin Company, the former using about 255 horse-power and the latter about Ioo horse-power. About half a mile below the dam above referred to is another dam affording about 8 feet head, and owned by the M. E. Pratt estate. This power operates a grist

*From U. S. Census, 1900.

$\dagger$ From report of Probate Judge. 
mill, cotton ginnery and ice factory, and the water wheel at that point has a rated capacity of 54 horse-power. About one mile above the dam of the Cotton Mill and Gin Company, there was formerly another dam 12 feet high, which afforded power for a cotton mill. This mill, however, was burned a number of years ago, and the dam has been allowed to go to ruin. It would probably afford 200 horse-power, or possibly a little more, should it be rebuilt.

There is also a dam about two miles below Prattville known as the Montgomery mill property. This dam is about 12 feet high and affords power for a grist mill and ginnery. Only a small portion of the available power is used. It could afford, easily, 250 horse-power if the proper wheels were installed.

\section{*BARBOUR COUNTY.}

NAME. POSTOFFICE.

INDUSTRY. H. P.

Hagler's Mill, Louiville, flour and grist mill............ 17

Carpenter's Mill, Louisville, flour and grist mill........... 15

H乞ffman's Mill, Clayton, flour and grist mill ............ 50

Hartman's Mill, Clayton, flour and grist mill ............ 10

Zorn Mills, Lodi, flour and grist mill ................ 8

William M. Wood, Bush, flour and grist mill............ 12

Will Stewart, White Oak springs, flour and grist mill....... 12

Winn's Mill, clayton, flour and grist mill............... 12

John White, Spivey, flour and grist mill ............. 10

Weston's Mill, Louisville, flour and grist mill............. 8

H. J. Turner, White Oak Springs, flour and grist mill....... 10

Spencer's Mill, Clayton, flour and grist mill............. 10

Perkin's Mill, Elamville, flour and grist mill ........... 12

Angus McSwain, White Oak Springs, flour and grist mill..... 12

William Johnson, Clayton, flour and grist mill........... 10

John M. Jenkins, Starhill, flour and grist mill........... 10

Sclomon's Mills, Solomon's Mills, flour and grist mill....... 25

Danner Mill, Elamville, flour and grist mill............. 12

William H. Chambers, Oateston, flour and grist mill........ 12

Wilson Deshazo, Cottonhill, flour and grist mill........... 16

\section{BIBB COUNTY.}

Scottsville Flour \& Grist Mill, Scottsville, flour and grist mill 30

Palmetto Flouring \& Grist Mill, Brierfield, flour and grist mill 30

Williams Grist Mill, Blocton, flour and grist mill........... 10

William S. Mathews, Data, flour and grist mill.......... 8

Six Mile Custom Mill, Six Mile, flour and grist mill........ 15

Mayfield Bros., Merte, lumber and timber mill............ 29

Scottsville, Wool Carder, Scottsville, woolen goods.......... 20

J. M. Battle, (Six Mile Creek), Six Mile, flour and grist mill. 50

W. C. Trott, (Six Mile Creek), Six Mile, cotton gin and grist

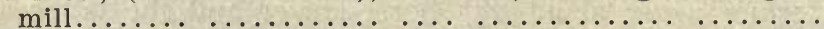

W. H. Thomas, (Six Mile Creek), Ashley, lumber and grist mill Dock Mahan, (Mahn's Creek), Brierfield, wool carder and

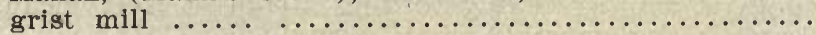

*From U. S. Census, 1900.

†From report of Probate Judge. 
NAMK.

POSTOFFICE.

INDUSTRY.

H. P.

Bessemer Land \& Improvement Co. (Schultz Crøek), Lopez,

mill and cotton gin $\ldots \ldots \ldots \ldots \ldots \ldots$....... 100

R. R. McCally, (Hills Creek), Blocton, gin, lumber and grist 30

E. M. Timbro, (Schultz Creek), Centerville, grist mill........ 30

F. H. James, (Haysoppy Creek), Centerville, grist mill....... 20

A. L. Elam, (Affonee Creek,) Áî́onee, grist mill........... 15

*BLOUNT COUNTY.

Logan Snead, Snead, flour and grist mill............... 10

E. B. Head, Gum Spring, flour and grist mill............... 16

E. R. Wood, Wynnville, flour and grist mill............ 8

Hendrick's Mill, Swansea, flour and grist mill........... 30

Jones M. Burns, Clarence, flour and grist mill........... 15

Wilson Adcock, Tidmore, flour and grist mill........... 10

G. M. D. Tidwell \& Sons, Tidwell, flour and grist mill....... 20

Alldridge \& Brother, Liberty, flour and grist mill.......... 10

Brittain Mill, Summit, flour and grist mill............ 20

Morris' Mill, Ensley, flour and grist mill............... 10

Rufus F. Wyatt, Bangor, flour and grist mill............ 10

Sam Mardis, Blountsville, flour and grist mill........... 60

Jno. H. Donahoo \& Geo. W. Darden, Rosa, lumber and timber 20

\section{BULLOCK COUNTY.}

Brooks' Mill, Mascotte, flour and grist mill............ 6 Union Springs Waste Mill, Union Springs, flour and grist mill 15 Chappell's Grisı Mill, Unton Springs, flour and grist mill..... 10 D. H. Mason, (McBride's Creek), Indian, lumber, gin and grist 20 From report of Probate Judge.

†Chas. Radfcrd, (Conecuh Creek), Union Springs, grist mill.. 10

\section{*BUTLER COUNTY.}

John W. Halso, Pigeon Creek, flour and grist mill........ 10

Glen Graham, Pontus, flour and grist mill............. 6

The Four Mile Mill, Greenville, flour and grist mill........ 10

The N. M. Rhodes Mill \& Mercantile Co., Shell, flour, grist and

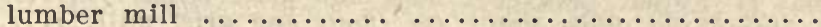

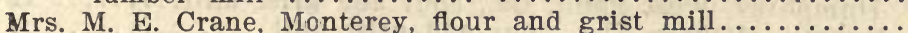

Rouse \& Whiddon, Greenville, flour and grist mill.........

CALHOUN COUNTY.

Joseph Francis, Cane Creek, flour and grist mill.......... 50

Richey Mill, Jacksonville, flour and grist mill.......... 20

Canada Grist Mill, Womack, flour and grist mill........... 16

Cold Water Mills, Cold Water, flour and grist mill......... 20

Read's Mill, Reads, flour and grist mill............... 60

* Luther Barton, Piedmont, flour and grist mill...............

W. F. McCulley, Oxford, flour and grist mill............ 20

A. McCurdy, White Plains, flour and grist mill............. 34

Morris Grist Mill, Morrisville, flour and grist mill.......... 18

Nisbet's Mill, Jacksonville, flour and grist mill.......... 30

James A. Weatherly, DeArmanville, flour and grist mill..... 8

*From U. S. vensus, 1900.

†From report of Probate Judge. 
NAME. POSTOFFICE.

INDUSTRY.

H. P.

Wood Milling lo., Ohatchee, flour and grist mill.......... 26

Davis \& Henderson, Piedmont, flour and grist mill................ 24

Hendon's Grist Mill, Iron City, flour and grist mill . . . . . . . . . 10

(Hughes' Saw Mill, Oxford, lumber and timber mill........ 28

F. M. Whiteside, (Choccolocco Creek), White Plains.....25 or 30

Downing \& Morris, (Choccolocco Creek), Choccolocco ....... 50

J. T. DeArman, (Choccolocco Creek), Anniston ........... 15

W. E. Mellon, (Choccolocco Creek), Oxford .............. 40

Lee's Mill, (Choccolocco Creek), Oxford.................. 30

T. G. Slaughter, (Choccolocco Creek), Oxford ........... 15

J. H. Savage, (Terrapin Creek), Anniston ............. 20

J. H. Savage, (Terrapin Creek), Anniston .............. 20

Frank Aderhold, (Nances Creek), Ladiga................. 20

John Ramagnand, (Champion Creek), Jacksonville.......... 15

James Crook, (Tallasseehatchee Creek), Jacksonville........ 10

W. J. Edmondson, (Tallasseehatchee Creek), Anniston ...... 30

W. A. Prickett, (Tallasseehatchee Creek), Alexandria....... 10

Beaty Estate, (Tallasseehatchee Creek), Alexandria........ 30

$\ddagger\{$ Peter Heifner, (Tallasseehatchee Creek), Alexandria.......... 15

James Aderhold, (Ohatchee Creek), Reads............... 20

Pleas. Martin, (Ohatchee Creek), Peekshill.............. 25

C. J. Wood, (Ohatchee Creek), Jacksonville............... 30

Wm. Thompson, (Ohatchee Creek), Peekshill............. 8

R. L. Treadway, (Tallasseehatchee Creek), Anniston, R. F. D 10

J. H. Francis, (Tallasseehatchee Creek) ............... 25

R. H. Cobb, (Tallasseehatchee Creek), Anniston............ 20

G. W. S. Loyd, (Cane Creek), Peaceburg................. 10

Mrs. Loyd, (Cane Creek), Peaceburg, gin ................ 6

Morris Mfg. Co., (Cane Creek), Morrisville, shops......... 30

E. G. Morris, (Cane Creek), Morrisville ................ 30

P. H. Brothers, (Cane Creek), Zula ................... 30

(J. H. Francis, (Cane Creek) ...................... 50

\section{*CHAMBERS COUNTY.}

D. E. M. Smith, Barber, flour and grist mill............ 24

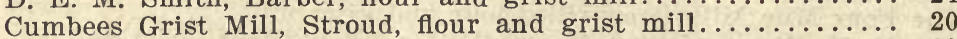

Thomas H. Fuller, Lafayette, flour and grist mill.......... 10

R. T. Humphrey, West Point, Ga., flour and grist mill....... 42

J. T. Hudson, Hickory Flat, flour and grist mill.......... 4

Wyche Robinson, Lafayette, flour and grist mill........... 16

Stephens' Mill, Driver, flour and grist mill............... 8

Ripville Mills, Wise, flour and grist mill............... 20

Charles F. Higins, Finley, flour and grist mill............. 20

J. E. Dixon, Lafayette, flour and grist mill ............. 10

Ratchford \& Tucker, Lafayette, flour and grist mill........ 10

Benjamin F. Knight, Lafayette, flour and grist mill......... 10

Wooddy \& Beall, Moorefield, flour and grist mill........... 6

Leverett's \& Abernathy's. Mill, Milltown, flour and grist mill.. 4

John B. Calhoun, Camphill, flour and grist mill.......... 8

G. L. Leverett, Lafayette, flour and grist mill . . . . . . . . 16

West Point Mfg. Co., West Point, cotton goods.............1,100

* From U. S. Census, 1900.

$\uparrow$ From report of Probate Judge.

from report of L. J. Morris. 


\section{CHEROKEE COUNTY.}

NAME.

POSTOFFICE.

INDUSTRY.

H. P.

(Shamblin \& Toles Mill, Broamtown, flour and grist mill..... 8

Chandler \& Stinson, Center, flour and grist mill............ 20

Shamblin \& Toles Mill, Broomtown, flour and grist mill..... 20

J. A. Lumpkin, Forney, flour and grist mill.............. 13

Hurleys Mill, Hurley, flour and grist mill............. 12

Tyre G. Craig, Grover, flour and grist mill............. 12

* Rush Mill, Lawrence, flour and grist mill..................... $10^{\circ}$

E. W. Ragdale, Spring Garden, flour and grist mill........ 30

W. F. Timmerman, Round Mountain, flour and grist mill... 8

M. E. Cohia, Cedar Bluff, flour and grist mill............. 24

M. J. Abernathy, Pleasant Gap, lumber and timber mill...... 15

Hurricane Creek Mfg. \& Min. Co., Spring Garden, cotton goods 65

W. A. Stinson, (Terrapin Creek), Center, gin, flour and grist 60

J. J. Scroggin, (Terrapin Creek), Coloma, gin, flour and grist 60

T. F. Stewart, (Terrapin Creek), Spring Garden, flour and grist 60

J. M. Adderhold, (Mill Creek), Piedmont, flour, grist and gin 40

M. L. Braswell, (Hurricane Creek), Pleasant Gap, flour \& grist 40

B. F. Newberry, (Yellow Creek), Round Mountain, flour, grist, and gin mill ............................ 40

E. Cobia, (Chattooga River), Cedar Bluff, flour, grist, and gin 60

$+\{$ R. A. Russell \& Co. (Chattooga River), Gaylesville, flour, grist

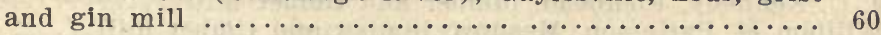

W. F. Henderson, (Mill Creek), Fullerton, flour, grist and gin 40

Rush \& Rinehart, (Chattooga River), Fullerton, flour, grist, gin 60

J. G. Toles, (Mill Creek), Broomtown, grist and gin mill.... 40

Elliott Bros., (North Spring Creek), Grassland, grist and gin 40

J. T. Webb \& Bros., (Spring Creek), Hurley, grist and gin mill 40

J. D. Jordan, (South Spring Creek), Noah, grist and gin mill.. 20

\section{*CHILTON COUNTY.}

James Dorming, Jemison, flour and grist mill........... 10

Mahan's Mill, Clanton, flour and grist mill.............. 20

W. W. Sansome, Adams, flour and grist mill............... 12

Honeycutt Mill, Jemison, flour, grist, lumber and timbor mill 20

\section{*CHOCTAW COUNTY.}

Pink Blackwell, Hinton, flour and grist mill............. 12

Aquilla Mills, Aquilla, lumber and timber mill............... 16

\section{*CLARKE COUNTY.}

Gate's Mill, Vashti, flour and grist mill.............. 30

Fleming's Grist Mill, Nealton, flour and grist mill............ 10

Dacy's Mill, Whatley, flour and grist mill................ 5

*From U. S. Census, 1900.

†From report of Probate Judge. 


\section{*CLAY COUNTY.}

NAME. POSTOFFICE.

INDUSTRY.

H. P.

Henry F. Smedley, Mellow Valley, flour and grist mill....... 15

Hezakiah Ingram, tatchett Creek, flour and grist mill...... 10

Allen P. Jenkins, Delta, flour and grist mill............. 14

Knight's Mill, Wesobulga, flour and grist mill........... 14

F. M. Munroe, Millerville, flour and grist mill............ 40

John R. Gilbert, Pinckneyville, flour and grist mill........ 8

Hodnett \& Co., Hat, flour and grist mill............... 10

Moses R. Watts, Dean, flour and grist mill.............. 6

Thomas J. Watts, Shinbone, flour and grist mill.......... 8

Bishop, Carpenter \& Co., Cherrry, flour and grist mill....... 10

Cockrell \& Mitchell, Goldburg, flour and grist mill.......... 14

McRairie, Gladney \& Co., Cherry, flour and grist mill....... 20

Virginia Whellen, Coleta, flour and grist mill........... 6

Stephens \& East, Delta, flour and grist mill.............. 4

Deberry \& Griffin, Fiatrock, flour and grist mill............. 15

Child's Mill, Swann, flour and grist mill.............. 5

James B. Brown, Pinckneyville, flour and grist mill......... 6

James J. Bachus, Fishhead, flour and grist mill............. 24

Brooks \& Handley, Hatchett Creek, flour and grist mill..... 8

Columbus Bell, Lineville, lumber and timber mill......... 10

J. C. Kennedy, Fishhead, lumber and timber mill........... 14

William M. Patterson, Meadow, lumber and timber mill....... 30

Ward \& Ford, Lineville, lumber and timber mill.......... 15

\section{*CLEBURNE COUNTY.}

J. T. \& E. W. Beason, Beasons Mill, flour and grist mill..... 10

W. M. Evans, Edwardsville, flour and grist mill........... 20

Robert Mill, Oaklevel, flour and grist mill.............. 16

Teague \& Co., Eudora, flour and grist mill............. 13

H. F. Alsabrook, Borden Springs, flour and grist mill...... 30

Buttram's Mill, Bucham, flour and grist mill............ 20

John A. Brown, Bell Mills, flour and grist mill.......... 16

John I. Burgess, Edwardsville, flour and grist mill......... 20

Wade H. Barnes, Muscadine, flour and grist mill.......... 4

J. W. Conner, Chulafinnee, flour and grist mill........... 6

Lyon \& Killebrue, flour and grist mill............... 34

W. G. Miligan, Oakfuskee, flour and grist mill.............. 8

James McMahan, Edwardsville, flour and grist mill........ 12

E. W. Pitchford, Oaklevel, flour and grist mill........... 15

William J. Thrash, Oakfuskee, flour and grist mill............ 6

Wade H. Barnes, Muscadine, flour and grist mill.......... 30

W. H. Tumlin \& D. S. Baber, Ai, flour and grist mill....... 16

\section{*COFFEE COUNTY.}

Levy Wise, Ino, flour and grist mill $\ldots \ldots \ldots \ldots \ldots \ldots \ldots \ldots$

Bell Mill, Dot, flour and grist mill...................... 8

Lenora F. Hildreth, Enterprise, flour and grist mill........ 17

Harper Flour Mills, Brockton, flour and grist mill.......... 4

F. M. Prestwood, Fresco, flour and grist mill.............. 20

McIntosh Mill, Eta, flour and grist mill................ 8

Wise's Lower Mill, Elba, flour and grist mill............ 12

Wise's Upper Mill, Elba, flour and grist mill.............. 10

Buck \& Co., Penn, lumber and timber mill.............. 50

*From U. S. Census, 1900. 


\section{*COLBERT COUNTY.}

NAME.

POSTOFFICE.

INDUSTRY.

I. $\mathbf{P}$.

George Martin, Allsboro, flour and grist mill............ 8

James Burns, Mand, flour and grist mill............. 4

Tuscumbia Mill, Tuscumbia, flour ana grist mill.......... 40

C. C. Hester, Tuscumbia, flour and grist mill........... 40

Chambee's Grist Mill, Tuscumbia, flour and grist mill....... 8

Dillard's Mills, Russellville, lumber and timber............ 12

Steenson's Mill, Sheffield, lumber and timber ............ 30

\section{*CONECUH COUNTY.}

George Stenson, Bonnette, flour and grist mill.......... 12

James B. Pate, Brooklyn, flour and grist mill............. 5

William M. Robinson, Brooklyn, flour and grist mill.......... 5

Jimson C. Cox, Gem, flour and grist mill............. 5

John N. Varner \& Chas. M. Varner, Herbert, flour and grist.. 10

James E. Wilson, Mount Union, flour and grist mill........ 20

Ransom H. Finley, Zern, flour and grist mill............. 8

G. G. Broker, Bowles, lumber and timber mill.......... 10

Cary \& Johnston, Brooklyn, lumber and timber mill........ 15

T. N. Piggott, Gravella, lumber and timber mill............ 40

Robinson Bros., Brooklyn, lumber and timber mill.......... 30

H. J. Robinson, Burnt Corn, lumber and timber mill....... 40

Henry Wills, Finklet, lumber and timber mill.......... 30

\section{*COOSA COUNTY.}

Miller's Mill, Bentleyville, flour and grist mill........... 20

Nolen's Mill, Darden, flour and grist mill................ 15

J. T. M. Hodnett \& O. P. Hodnett, Equality, flour and grist mill 12

W. N. Neighbors, Goodwater, flour and grist mill.......... 23

Smith's Mill, Nixburg, flour and grist mill............. 10

George P. Waits, Rockford, flour and grist mill.......... 8

Crawford Mill, Rockford, flour and grist mill........... 4

Lawson Grist and Saw Mill, Rockford, lumber and timber mill 36

\section{*COVINGTON COUNTY.}

A. J. Fletcher, Andalusia, flour and grist mill........... 10

Uatu Grist Mill, Andalusia, flour and grist mill.......... 10

William Sharp, Ealums, flour and grist mill............. 10

Davis B. Gantt, Gantt, flour and grist mill.............. 12

C. E. Rawls, Gantt, flour and grist mill............... 10

Dorsey's Mill, Glasiasko, flour and grist mill............. 10

James Aplin, Green Bay, flour and grist mill............ 20

William Watkins, Liberty Hill, flour and grist mill........ 8

Kearsey's Mill, Redlevel, flour and grist mill............. 5

Ephram F. Lassiter, Rosehill, flour and grist mill........... 10

Thomas Saw Mill, Redlevel, lumber and timber mill........ 25

Simmons Mill, Beck, lumber and timber mill............. 40

J. A. Prestwood, Jr., Andalusia, lumber and timber mill...... 40

George W. Lee, Rat, lumber and timber mill............... 20

Buck Creek Mill, River Falls, lumber and timber .......... 80

J. F. Guthrie, Vera Cruz, lumber and timber mill......... 25

Gunter's Mill, Andalusia, lumber and timber mill........... 40

Gunter's Saw Mill, Gantt, lumber and timber mill.............. 15

Gantt's Mill, River Falls, lumber and timber mill......... 70

Pollard Gantt, Searight lumber and timber mill........... 35

Davis B. Gantt, Gantt, lumber and timber mill............ 40

N. B. Dixon, Mason, lumber and timber mill............. 60

Bartlett \& Barker, lumber and timber mill.............. 60

*From U. S. Census, 1900. 


\section{*CRENSHAW COUNTY.}

POSTOFFICE.

INDUSTRY.

H. $\mathbf{P}$.

E. P. Lasseter, Bullock, flour and grist mill............. 8

G. B. Morgan, Bullock, flour and grist mill............. 15

Folmar's Mill, Goshen, flour and grist mill............... 8

N. Skipper, Honoraville, flour and grist mill .................. 10

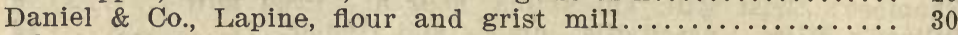

John S. Marsh, Rutledge, flour and grist mill............ 20

G. B. Sasser, Luverne, flour and grist mill.............. 15

*CULLMAN COUNTY.

Joseph W. Hyatt, Baileyton, flour and grist mill.......... 10

Miles Humphries, Baileyton, flour and grist mill........... 4

D. H. Laney, Battleground, flour and grist mill........... 6

Robert J. Waldrop, Cranehill, flour and grist mill........ 20

Andrew J. Miller, Summit, flour and grist mill............ 6

\section{*DALE COUNTY.}

Archer McCall, Candy, flour and grist mill............. 10

Floyd Mill, Dothan, flour and grist mill.............. 10

Lewis Mill, Clopton, flour and grist mill ............... 15

Murphy Mill, Dothan, flour and grist mill............ 5

Maunds Corn Mill, Ewells, flour and grist mill............ 10

Pope's Mill, Grimes, flour and grist mill................ 60

Charles Thrower, Kleg, flour and grist mill.............. 16

Daniel McSwean, Ozark, flour and grist mill............ 20

Preston's Mill, Peach, flour and grist mill.............. 20

The Kelley Grist Mill, Pinckard, flour and grist mill....... 150

Atkinson's Saw Mill, Newton, lumber and timber mill....... 16

J. F. Bell, Daleville, lumber and timber mill.............. 22

*DALLAS COUNTY.

Calhoun's Mill, Carlowville, flour and grist mill........... 10

Ivey \& Williams, Morrowville, flour and grist mill........ 8

DEKALB COUNTY.

L. D. Wooten, Blake, flour and grist mill............. 8

J. D. Hall, Chavies, flour and grist mill.................... 10

J. S. Ward, Chumley, flour and grist mill................ 12

Kean \& Warren, Cordell, flour and grist mill............ 20

Swindell's Mill, Cotnam, flour and grist mill................ 12

Griffin's Mill, Cotnam, flour and grist mill.............. 12

Emeline Clayton, Crossville, flour and grist mill........... 6

Swader's Mill, Dekalb, flour and grist mill.............. 15

James Clark, Eula, flour and grist mill................ 15

* David J. Harper, Floy, flour and grist mill.............. 3

Elrod's Grist Mill, Flay, flour and grist mill.............. 4

Davis Mill, Fort Payne, flour and grist mill............. 16

Thomas F. Everett, Luna, flour and grist mill............ 8

Elrod's Mill, Geraldine, flour and grist mill.............. 30

Pruitt's Mill, Skirum, flour and grist mill.............. 12

Lebanon Flour \& Grist Mill, Lebanon, flour and grist mill... 36

Robert F. Ellison, Mentone, flour and grist mill......... 25

Ellic Ellsworth, Opnir, flour and grist mill............... 6

Warren's Grist Mill, Portersville, flour and grist mill........ 12

*From U. S. Census, 1900. 
YAME.

POSTOFFICE.

IXDUSTRY.

H. P.

John F. Williams, Rains, flour and grist mill............. 8

Edward W. Williams, Rains, flour and grist mill.......... 6

McGee's Mill, Sand Rock, flour and grist mill.............. 5

Charles G. Matheny, Sauty Mills, flour and grist mill...... 20

Dixie Mills, Sulphur Springs, flour and grist mill.......... 10

* Phillips' Mill, Valleyhead, flour and grist mill............. 4

The Roberts Mill Co., Collinsville, four and grist mill...... 25

W. E. Brown \& Son, Sulphur Springs, lumber and timber mill.. 15

James M. Durham, Chavies, Iumber and timber mill.......... 16

William C. Hill \& Co., Blanche, lumber and timber mill..... 40

D. D. Hughes, Hughes, lumber and timber mill........... 15

Ward, Pickens \& Co., Dawson, lumber and timber mill......... 15

John A. Davis, (Wills Creek), Fort Payne, grist mill and gin

M. S. Brown and W. C. Thomas, (Lookout Creek), Sulphur

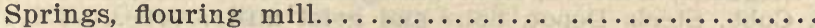

$+\{$ D. D. Hughes, (Wills Creek), Hughes $P$. O.; flour \& grist mill P. M. Frazier, (Wills Creek), Lebanon, flour and grist mill.. S. D. Warren, (Wills Creek), Lebanon, flour and grist mill.... Grif. Elrod, (Town Creek), South Hill, flour and grist mill.... Durham \& Co., (Town Creek), Chavies, flour, grist \& saw mill

*ELMORE COUNTY.

E. \& H. T. Andrews, Channahatchee, flour and grist mill.....

Benjamin Spigener, Elmore, flour and grist mill............

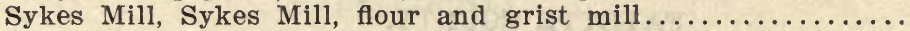

John C. Birt (Lancaster Old Mill,) Tallassee, flour and grist

Freeman's Grist Mill, Tallassee, flour and grist mill.........

J. J. Benson, Kowaliga, lumber and timber mill............

J. T. Rogers, Spigners, lumber and timber mill............... (From Chapter III.)

\$allassee Falls Mfg. Co., (Tallapoosa River,) Tallassee, cotton

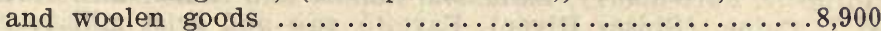

Montgomery Power Co. (Tallapoosa River), Tallassee, electric

transmission to Montgomery, Ala...............5,600

*ESCAMBIA COUNTY.

Bradley Mill, flour and grist mill .................... 10

S. S. Uverstreet, Roberts, flour and grist mill........... 20

James F. Douglas, Mason, lumber and timber ........... 25

*From U. S. Census, 1900.

\#This is the same company that is now organized under the name of the Mt. Vernon Woodbury Cotton Duck Company, with office at Montgomery, Ala. 


\section{ETOWAH COUNTY.}

NAME.

POSTOFFICE.

INDUSTRY.

H. $P$.

(Wesson Mills, Attalla, flour and grist mill............. 25

Cox \& Brother, Avery, flour and grist mill................... 6

B. H. Rogers, Etowahton, flour and grist mill................ 40

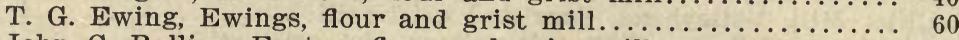

John C. Rollins, Fenton, flour and grist mill................. 8

Reese Mill, Hill, flour and grist mill...................... 10

John H. Helms, Ballplay, flour and grist mill.............. 6

Ford \& Sibert's Mill, Hokes Bluff, flour and grist mill....... 30

* $\{$ Morgan \& Cochran, Keener, flour and grist mill............. 8

W. J. Harris, Nix, flour and grist mill.................... 12

John B. Burns, Seaborn, flour and grist mill............. 8

A. B. Stephens, Seaborn, flour and grist mill............. 8

W. H. Cobb, Steels Depot, flour and grist mill.............. 20

P. C. Turner, Walnut Grove, flour and grist mill............ 30

P. C. Turner, Walnut Grove, woolen goods............... 13

W. M. Brothers \& Son, Gallant, woolen goods.............. 8

(Gadsden Times-News, Gadsden, printing and publishing...... 4

「J. M. Morague, (Big Wills Creek), Gadsden, grist mill....... 100

Wm. McClendon, (Big Wills Creek), Attalla, grist mill........ 40

$\nmid\{$ - Griffith, (Big Wills Creek), Keener, grist mill.......... 35

Bob Rigers, (Big Canoe Creek), Gadsden, grist mill.......... 75

(Tom Ewing, (Cane Creek), Gadsden, grist mill........... 40

FAYETTE COUNTY.

「Rodolphus Cotton, Bankston, flour and grist mill.......... 20

I D. G. Hester, Covin, flour and grist mill................... 12

* John W. Anthony, Glenallen, flour and grist mill............ 30

Landon Miles, Hester, flour and grist mill.................. 13

Bishop Emick, Rena, lumber and timber mill.............. 40

Phillip N. Fortenberry, Bankston, lumber and timber mill....... 8

W. L. Caine, (Sipsey River), Fayette, saw and grist mill..... 40

T. E. Newton \& Bro., (S1spey River), Fayette, saw and grist 40

Licurgas Ray, (Luxapelila Creek), Montcalm, saw and grist.. 30

John Barnes, (Luxapelila Creek), Covin, gin and grist mill.. 30

E. Bishop, (Luxapelila Creek), Rainy, saw, gin and grist mill 30

John Williams, (Luxapelila Creek), Covin, gin and grist mill.. 30

Washington Hubbert, (Shirley Creek), gin and grist mill... 10

Gilpin \& Jones, (Shirley Creek), saw, gin and grist mill..... 16

Jones \& Jones, (Shirley Creek), Hugent, saw, gin \& grist mill 20

P. N. Fortenberry, (Davis Creek, Bankston, saw, gin and grist 8

G. H. White, (Davis Creek), Davis Creek, saw, gin and grist 16

$+\{$ J. W. Blackburn, (Davis Creek), Davis Creek, saw, gin \& grist 18

M. I. Barnette, (Davis Creek), Ridge, saw, gin and grist mill.. 20

Dolphus Cotton, (Clear Creek), Bankston, saw, gin and grist 16

M. Miller, (Clear Creek), Bankston, saw, gin and grist mill..

John G. Kizer, (North River), Berry Station, saw, gin \& grist 40

Marshall Jones, (Bear Creek), Bear, saw, gin and grist mill 20

R. G. Walker, (Bear Creek), Bear, saw, gin and grist mill.... 24

Landon Miles, (Stewart Creek), Hester, grist mill.......... 12

J. T. McCaleb, (Mountain Creek), New River, grist mIII..... It

W. A. Ayers, (Beaver Creek), Fayette, gin and grist mill...... 12

G. W. Gray, (Boxes Creek), Stough, grist mill............ 16

Miles Whitson, (Clear Creek), Handy, grist mill........... 12

Bud Wade, (Hollingsworth Creek), New River, grist mill... 12

*From U. S. Census, 1900.

$\dagger$ From report of Probate Judge. 


\section{*FRANKLIN COUNTY.}

Helm's Mill, Belgreen, flour and grist mill............... 6

M. J. Height, Baggett, flour and grist mill............ 10

James McNair, Kirby, flour and grist mill.............. 20

Andrew Posey, Igoburg, flour and grist mill........... 24

Thomas Watson, Phil Campbell, flour and grist mill......... 20

S. T. Bonds, Pleasant Site, flour and grist mill............ 80

Jes. S. Scott, Russellville, flour and grist mill........... 10

Sparks Mill, Underwood, flour and grist mill............ 10

John T. McAlister, Phil Campbell, lumber and timber mill.... 10

*GENEVA COUNTY.

Avant's Mill, Geneva, flour and grist mill.............. 15

Lowry's Mill, Geneva, flour and grist mill............. 10

Bell's Mill, Fadette, flour and grist mill................ 15

W. J. Keith and R. Y. Daniels, Geneva, flour and grist mill.. 15

Clark's Grist Mill, Highnote, flour and grist mill.......... 4

Underwood's Grist Mill, Sanders, flour and grist mill........ 20

Condry's Grist Mill, Whitaker, flour and grist mill......... 15

John T. Coleman, Geneva, lumber and timber mill........... 30

Clark Bros. \& Co., Wicksburg, lumber and timber mill....... 10

Wilson Deshoga, Dundee, lumber and timber............. 15

Nathan Hall, Dotham, lumber and timber............ 20

HALE COUNTY.

(William Steward, Fivemile, flour and grist mill........... 8

William A. Avery, (Five-Mile Creek), Five-Mile, flour and grist 10

J. H. Payne \& Co., Ingram, flour and grist mill........... 10

* M. M. Avery, Havanna, flour and grist mill.............. 15

Pickens Mill, Greensboro, lumber and timber mill......... 15

Greensboro Carriage \& Wagon Shops, Greensboro, carriages and

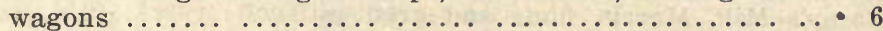

Richardsons Mills, (Five-Mile Creek), Five-Mile, grist mill

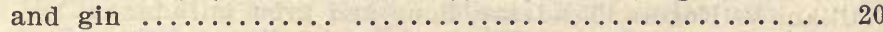

J. H. Payne's Mill, (Five-Mile Creek), Havana, grist and gin. 20

Avery's Mill, (Five-Mile Creek), Havana, grist mill and gin.. 25

J. A. Stephenson, (Prairie Creek), Newbern, grist mill \& gin.. 20

(Irwin \& Martin, (Big Creek), Greensboro, grist mill and gin.. 25

\section{*HENRY COUNTY.}

Kennedy's Mill, Shorterville, flour and grist mill.......... 8

Joshua A. Hart, Granger, flour and grist mill........... 15

Jeffcoat Mill, Gordon, flour and grist mill................. 8

Blacksheer \& Saunders, Haleburg, flour and grist mill...... 25

Cumming's Mill, Bush, flour and grist mill.. ............. 20

Joe Baker, Hadland, flour and grist mill ................ 27

Badiford Grist Mill, Little Rock, flour and grist mill........ 15

Blackshe \& Sanders, Haleburg, flour and grist mill......... 15

John L. Smith, Ashford, flour and grist mill............... 13

Mark Shelley, Balkum, flour and grist mill............ 6

Singleterry's Water Mill, Kinsey, lumber and timber........ 27

J. P. Williams \& Co., Columbia, lumber and timber.......... 25

*From U. S. Census, 1900.

†From report of Probate Judge. 


\section{*JACKSON COUNTY.}

NAME.

POSTOFFICE.

INDUSTRY.

H. P.

Moody's Flouring Mill, Kyles, flour and grist mill......... 40

George $W$. Brown, Kosh, flour and grist mill.............. 8

J. F. Bell, Maxwell, flour and grist mill .................. 4

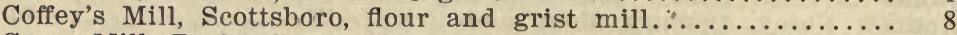

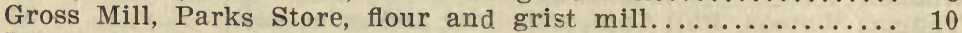

Hackworth's Mills, Bolivar, flour and grist mill............... 8

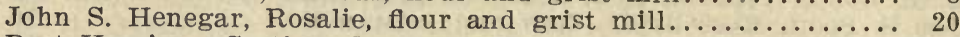

Bort Harrison, Section, flour and grist mill................. 6

W. A. Howell, Hollytree, flour and grist mill $\ldots \ldots \ldots \ldots \ldots \ldots \ldots$.

Mathew's Grist Mill, Carns, flour and grist mill................. 10

Page's Mill, Woodville, flour and grist mill.................. 6

Paint Rock Milling Co., Paint Rock, flour and grist mill....... 8

Reid \& Prince, Estillfork, flour and grist mill........... 20

David H. Starkey, Kosh, flour and grist mill............. 8

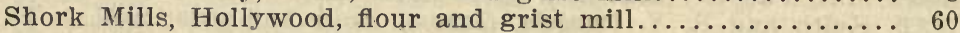

Cagle Mill, Oakley, flour and grist mill.................. 12

John Thomas, Pisgah, flour and grist mill.............. 20

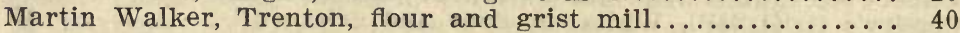

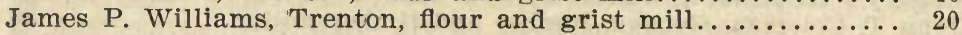

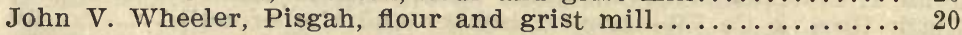

Charles W. Brown, Glenzaida, lumber and timber mill........ 25

J. N. Gonce, Anderson, lumber and timber mill........... 20

Melton Morris, Daugherty, lumber and timber mill......... 12

David M. Starkey, Kosh, lumber and timber mill........... 20

Tomon Shingle Mill, Culver, lumber and timber mill.......... 10

\section{*JEFFERSON C6OUNTY.}

J. M. Landrum, Pinson, flour and grist mill ............ 20

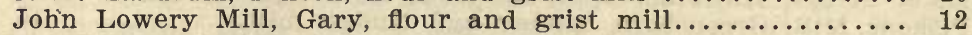

Hendon's Corn Mill, Trussville, flour and grist mill........... 10

Posey's Mill, Morris, flour and grist mill.................. 20

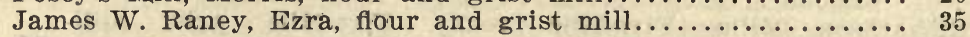

William B. Rogers, Toadvine, flour and grist mill........... 32

G. W. Underwood, Argo, flour and grist mill $\ldots \ldots \ldots \ldots \ldots \ldots \ldots \ldots$

William J. Wedgworth, Cardiff, flour and grist mill................ 10

W. W. Woodruff, Adamsville, flour and grist mill ........ 8

W. M. Self, Oneonto, flour and grist mill............... 15

William M. Phillip, Greene, flour and grist mill.......... 40

Hurst \& Johnson, Pinson, lumber and timber mill......... 18

James W. Raney, Ezra, woolen goods ................ 35

\section{LAMAR COUNTY.}

John H. Cantrell, Pharos, flour and grist mill........... 15

Claborn E. Carter, Detroit, flour and grist mill.................... 12

Kirk's Mill, (Yellow Creek), Sizemore, flour and grist mill.... 8

Mote's Mill, (Beaver Creek), Guin, flour and grist mill....... 6 Jóhn T. Moore, (Yellow Creek), Vernon, flour and grist mill.. 35

.H. W. Miller, (Luxapelila Creek), Millport, flour and grist mill 20

Stanford Mills, Detroit, flour and grist mills............. 12

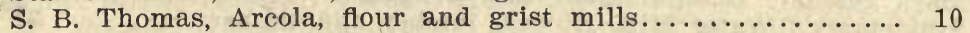

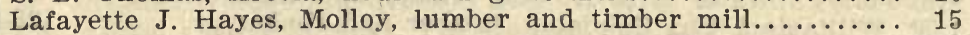

H1ram Hollis, Vernon, lumber and timber mill............ 35

Dr. Wm. H. Kennedy, Kennedy, lumber and timber mill..... 50

S. B. Thomas, Arcola, lumber and timber mill..............

15

*From U. S. Census, 1900. 
NAME.

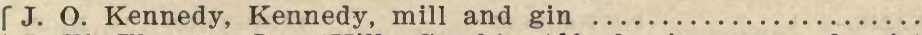
J. W. Thomas, Jr., (Hills Creek), Alfred, gin, saw and grist W. M. Thomas, (Hills Creek), Alfred, gin, saw and grist mill Osborn \& Hill, (Yellow Creek), Blowhorn, gin, saw and grist D. M. Hollis, (Beaver Creek), Beaverton, gin, saw and grist B. G. Boman, (Yellow Creek), Vernon, gin, saw and grist mill A. A. Mathews, (Yellow Creek), Arcola, gin, saw and grist mill W. L. Morton, (Yellow Creek), Vernon, gin, saw and grist mill Penning Bros., Baxter, gin, saw and grist mill...............

\section{*LAUDERDALE COUNTY.}

William M. Thornton, Rogersville, flour an grist mill......... James A. Bevis, Threet, flour and grist mill...............

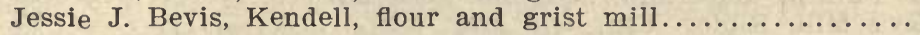
George M. Bretherick, Hines, flour and grist mill...........

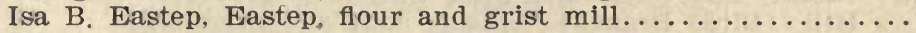
Ingram Brothers, Anderson, flour and grist mill.......... Thomas D. Pruitt, Pruitton, flour and grist mill.............. Sharpe's Mill, Florence, flour and grist mill............... Nancy Williams, Lexington, flour and grist mill.............

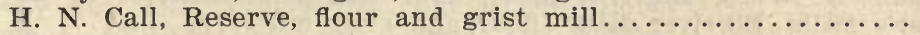
Chandler \& Chittam, Oliver, flour and grist mill.............

LAWRENCE COUNTY.

Burrell \& Casteel, Progress, flour and grist mill...........

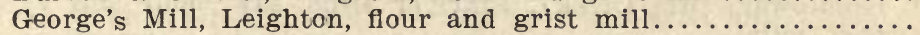
Jones' Estate, Kinlock, flour and grist mill...............

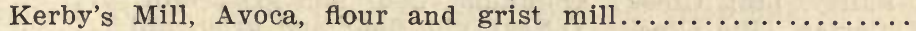

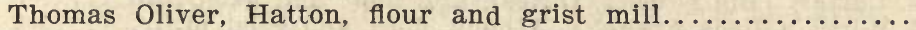
John S .Stephenson \& Co., Kinlock, flour and grist mill........

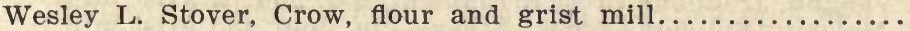
Terry \& Terry, Courtland, flour and grist mill.............

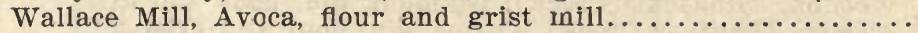
W. M. Willingham, Camp Spring, lumber and timber mill.... H. C. McClannaher, (Town Creek), Mount Hope, grist mill.... John S. Stephenson, (Sipsey River,) Moulton, flour and grist Ben F. Masterson, (Big Nance Creek), Moulton, grist mill....

W. G. Hamilton, (Big Nance Creek), Pitt, grist mill......... J. M. Key, (Brushey Creek), Pool, grist mill............... W. L. Stover, (Flint Creek), Oakville, flour and grist mill.... B. A. Casteel, (Flint Creek), Sewickley, flour and grist mill..

LEE COUNTY.

Shelton's Mill, Opelika, flour and grist mill...............

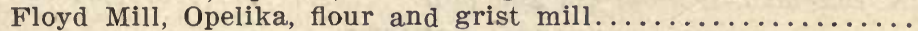
George W. McKinnon, Yale, flour and grist mill.............. Vaugh Mill, Loachapoka, flour and grist mill............... N. G. Macon, (Reed Creek), Loachapoka, flour and grist mill..

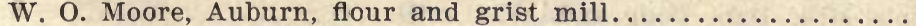
W. K. Meadows, (Halawochee Cr.), Hattie, flour and grist mill James Crosby, Osanippa, flour and grist mill .............. Benjamin F. Stripling, Yale, lumber and timber............... W. W. Wright, (Chewacla Creek), Auburn, not in use now......

W. W. Wright \& Geo. P. Harrison, Opelika, (Saugahatchee Cr.) H. J. Spratling, (Frazer Creek), Opelika, grist mill......... 25 B. F. Meadows, (Halawochee Creek), Opelika, grist mill.......

*From U. S. Census, 1900.

$\dagger$ From report of Probate Judge. 


\section{LIMESTONE COUNTY.}

NAME. POSTOFHICE.

INDUSTRY.

H. P.

(Weatherford Bros., Elkmont, flour and grist mill..........

Carter's Mill, Athens, flour and grist mill................ 16

Dupree \& Stepp, Mount Rozell, flour and grist mill............ 25

Haye's Grist Mill, Mooresville, flour and grist mill............ 15

T. M. Holmes, Elkmont, flour and girst mill............... 12

John M. Head, Pettusville, flour and grist mill............. 8

Nancy Haney, Legg, flour and grist mill................... 20

Edward G. Hampleton, Goodsprings, flour and grist mill....... 15

Thomas D. Hastings, Elkmont, flour and grist mill......... 5

James L. Lamar, Goodsprings, flour and grist mill........... 8

Eugene Parham, (Piney Creek), Athens, flour and grist mill.. 8

M. A. Phillips, Shoalford, flour and grist mill............ 12

Ripley's Mill, Ripley, flour and grist mill................ 15

George Vassar, Lax, flour and grist mill.................. 8

Witty's Mill, (Birds Branch), Athens, flour and grist mill.... 15

William J. Woodfin, Pettusville, flour and grist mill........ 15

Pioneer Mill, Mount Rozell, flour and grist mill............ 20

A. P. Andrews, Elkmont, flour and grist mill........... 8

William N. Webb, Elkriver Mills, flour and grist mill...... 12

Baker's Mills, Elkriver Mills, flour and grist mill........... 8

Allison Miller, Rowland, flour and grist mill................ 10

Grisham Bros., Elkriver Miıls, lumber and timber.......... 40

Grisham Bros., Elkriver Mills, carriages and wagons....... 40

( L. C. Hightower, (Big Creek), Elkriver Mills, saw, flour and

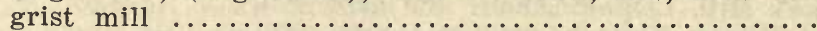

Wm. Bailey, (Big Creek), Quidnunc, flour and grist mill....

J. W. Carter, (Big Creek), O'Neal, gin, flour and grist mill....

M. J. Witty, (Birds Branch), Athens, flour and grist mill......

J. C. Vaughn, (Sulphur Creek), Elkmont, gin, flour \& grist mill

R. B. Malone, (Sulphur Creek), Athens, gin, flour and grist mill

Wm. Woodfin, (Ragsdale Creek), Elkmont, gin, flour and grist

J. W. Carter, (Panther Creek), Carter, gin, flour and grist mill

John Carroll, (Leslie Creek), Centerhill, gin, flour and grist mill

Wm. Davidson, (Limestone Creek), Lax, gin, flour and grist mill

R. M. Clem, (Piney Creek), Fairmount, gin, flour and grist mill

Eugene Parkam, (Piney Creek), Athens, gin, flour and grist mill

W. M. Hayes, (Limestone Cr.), Mooresville, gin, flour and grist

W. H. Roberts, (Sugar Creek), Athens, gin, flour and grist mill

W. H. Marbut, Goodsprings, gin, flour and grist mill.........

\section{LOWNDES COUNTY.}

${ }^{*}$ G. B. Holley, Lowndesboro, flour and grist mill..........

$\dagger W$. N. Bozeman, Benton, gin and mill................

*MADISON COUNTY.

Fannie J. Ridley, Haden, flour and grist mill..............

D. L. Middleton Water Mill, Gurley, flour and grist mill......

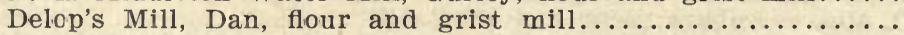

Hardy Keel Water Mill, Gurley, flour and grist mill.........

Annie M. Taylor, Hazelgreen, flour and grist mill...........

Bellfactory Mill, Huntsville, flour and grist mill............

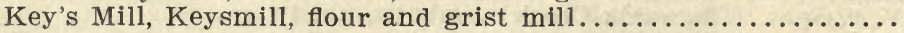

William S. Russell, Madison Station, flour and grist mill......

Chas. F. Rountree, Maysville, flour and grist mill..........

William S. Garvin, Monrovia, flour and grist mill............

A. D. and W. E. Rogers, Newmarket, flour and grist mill.....

Butler Mill Co., Poplarridge, flour and grist mill...........

Payne \& Miller, Huntsville, flour and grist mill............

Martin's Grist Mill, Huntsville, flour and grist mill..........

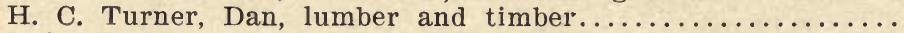

Daily Mercury, Huntsville, printing and publishing..........

*From U. S. Census, 1900.

$\dagger$ From report of Probate Judge. 
*MACON COUNTY.

NAME. POSTOFFICE.

INDUSTRY.

H. 1 .

H. H. Robinson, Loachapoka, flour and grist mill........ 4

M. W. Glass, Societyhill, flour and grist mill........... 8

J. O. H. Perry, Tuskegee, flour and grist mill........... 20

\section{MARION COUNTY.}

The Carter Mill, Ur, nour and grist mi $1 \ldots \ldots \ldots \ldots \ldots \ldots \ldots \ldots$ Bexar Mercantile Co., Bexar, flour and grist mill............

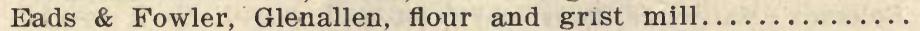

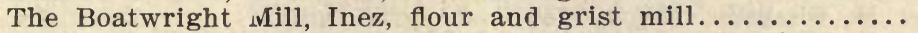
Samuel A. \& Wm. V. Read, Eldridge, flour and grist mill...... Jasper N. Green \& sons, Brilliant, flour and grist mill....... Elishu Vickery, Winfield, flour and grist mill.............

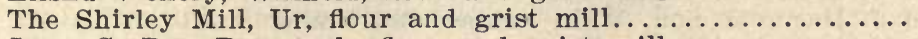
Jesse G. Poe, Bearcreek, flour and grist mill............. Bull, Atkins \& Donaldson, Haleysville, fiour and grist mill.... Buttahatchee Mill Co., Haleysville, lumber and timber....... John Cumens, Haleysville, lumber and timber ............. Kelly Saw Mill, Haleysville, lumber and timber............

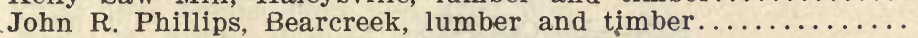

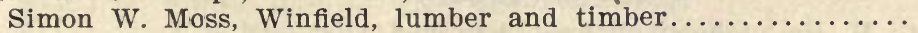
The Powell Mill \& Wool Carder, Duffey, woolen goods........ Albert J. Hamilton, (Williams Creek), Hamilton, flour and grist W. C. Gann, (Sipsey Creek), Bexar, flour and grist mill....... Q. Northington, (Sipsey Creek), Hamilton, flour and grist mill Crane \& Riggs, (Sipsey Creek), Delhi, flour and grist mill...... T. L. Shotts, (Bull Mountain Creek), Shottsville, flour and grist I. J. Loyd, (Bull Mountain Creek), Bull Mountain, flour and grist D. F. Ballard, (Williams Creek), Hamilton, flour and grist mill James P. Pearce, (Buttahatchee River), Pearce's Mill, flour and

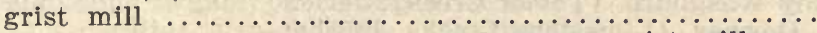

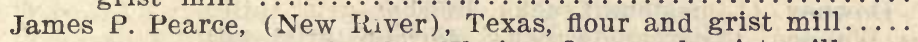
J. C. Carter, (Woods Creek), Elmira, flour and grist mill..... James Young, (Cantrell Mill Creek), Hamilton, flour and grist W. J. Wright, (Barnesville Mill Creek); Barnesville, flour and grist mill

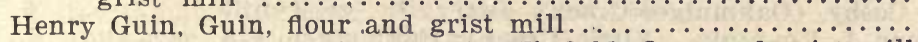
Tucker Moss, (Luxapelila Creek), Winfield, flour and grist mill D. G. Morrow, (Woods Creek), Elmira, flour and grist mill.....

\section{*MARSHALL COUNTY.}

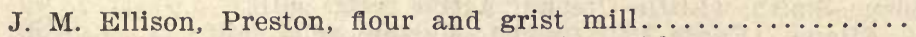

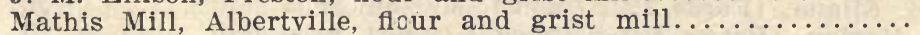
James B. Powell, Columbus City, flour and grist mill.......... James F. Prentice, Arab, flour and grist mill.............

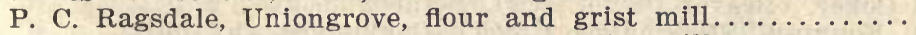
James P. Smith, Warrenton, flour and grist mill...........

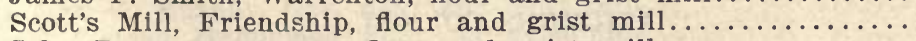

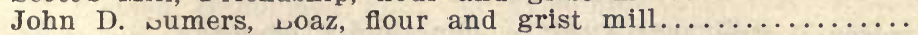

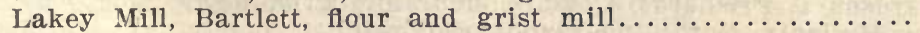
George E. Whisnant \& Son, Oleander, flour and grist mill..... I. G. Gross, Columbus City, flour and grist mill........... Walker \& Fowler Mills, Friendship, flour and grist mill...... William J. Copelan, Diamond, flour and grist mill........... James Wm. Barclay, Woodville, flour and grist mill........ The Winston Mil, Meltonsville, flour and grist mill......... W. G. Smith Estate, Sidney, flour and grist mill............ Jas. M. Selvage, Grant, flour and grist mill.............. 


\section{MARENGO COUNTY}

NAME.

POSTOFFICE.

INDUSTRY.

H. $P$.

*Rhodes Mill, Sweetwater, flour and grist mill........... 12

*MOBILE COUNTY.

N. Q. Thompson, Citronelle, flour and grist mill......... 10

H. Brannan \& Son, Pierce, lumber and timber................ 30

T. A. Hatter \& Son, Creola, lumber and timber............ 75

Littleton Lee, Pierce, lumber and timber.................6 60

*MONROE COUNTY.

J. B. Solomon, Manistree, flour and grist mill............ 15

James H. Simpson, Mexia, flour and grist mill................ 10

Benjamin Johnson, Hollinger, flour and grist mill.............. 15

Andrew Bohanon, Franklin, flour and grist mill............... 15

David J. Hatter \& Son, Wait, lumber and timber........... 60

David J. Hatter \& Son, Wait, lumber and timbèr............ 20

C. C. Yarbrough, Monroeville, lumber and timber ........... 20

*MONTGOMERY COUNTY.

Daniel's Mill, Sellers, flour and grist mill............. 25

Montgomery Cotton Mill, Montgomery, cotton goods ......... 35

MORGAN COUNTY.

*Sarah M. McCutcheon, Briscoe, flour and grist mill........ 10

PERRY COUNTY.

Henry C. Nichols, (Dobynes Creek), Theo, flour and grist mill. 20

Mary G. Wallace, Marion, flour and grist mill............ 4

Hodger's Mill, Newbern, flour and grist mill............... 15

*

W. F. Moore, Marion, flour and grist mill.............. 4

Downey's Saw Mill, Greensboro, lumber and timber ........ 15

Stevenson's Saw \& Water Mills, Newbern, lumber and timber.. 20

Lucindy Washburn, (Taylor Creek), Jericho, lumber and timber 18

W. T. Downey, (Limestone Creek), Folsom, grist mill......... 6

James Wallace, (Legroane Creek), Jericho, grist mill....... 8

Dr. J. B. Tucker, (Taylors Creek), Jericho, grist mill ......... 6

Lucindy Washburn, (Taylors Creek), Jericho, grist mill....... 8

S. M. Bolling, (Branch of Oakmulgee Cr.), Pinetucky, grist mill 8

C. C. Cosby, (Oakmulgee Creek), Perryville, grist mill........ 8

Thomas J. Fountain, (Little Creek), Oakmulgee, gin, saw and

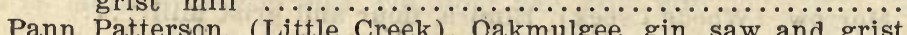

Sarah Fountain, (Little Creek), Oakmulgee, gin, saw and grist

Thaddeus Smith, (Little Creek), Active, grist mill...........

W. M. Eiland, (Fords Mill Creek), Marion, grist mill..........

J. F. Morton, (Potato Patch Creek), Levert, grist mill.........

Elijah Smith, (Beaver Creek), Bliss, grist mill.............

Noah Coker, (Beaver Dam Creek), Bethlehem, grist mill......

'W. A. Fountain, (Oakmulgee Creek), Oakmulgee, rice mill.... 10

*PICKENS COUNTY.

Richardson \& Prichards, Coalfire, flour and grist mill.......

James Mullenix, Gordo, flour and grist mill...............

H. B. \& A. W. Latham, Carrollton, flour and grist mill.......

Slaughter's Mill, Raleigh, flour and grist mill...............

W. A. Kerr, Reform, lumber and timber ...................

*From U. S. Census, 1900.

†From report of Probate Judge. 
*PIKE COUNTY.

$\begin{array}{llll}\text { NAME. } & \text { POSTOFFICE. } & \text { INDUSTRY. P. }\end{array}$

M. J: Youngblood, Youngblood, flour and grist mill.........110

William F. Ingram, Josie, flour and grist mill........... 20

Nancy Cotton, (Cotton's Mill), Milo, flour and grist mill....... 12

Ely Dees \& J. D. Murphee, Pronto, flour and grist mill....... 20

George W. King, Gosnen, flour and grist mill............. 30

The Lewis Mill, Rodney, flour and grist mill............. 24

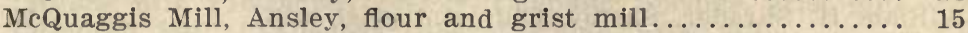

George F. Williams, Tatum, flour and grist mill........... 4

Slatting's Grist Mill, Henderson, flour and grist mill....... 25

P. A. Motia, Wingard, flour and grist mill............. 8

Bowden \& Daughtry, Tennille, flour and grist mill.......... 16

William E. Brown, Josie, flour and grist mill........... 10

G. B. Howard, Goshen, flour and grist mill............. 20

\section{*RANDOLPH COUNTY.}

W. W. Dobson, Wedowee, flour and grist mill............. 20

J. H. White \& Z. N. Lipham, Clack, flour and grist mill....... 11

Mrs. Georgia Gibos, Wedowee, flour and grist mill.......... 10

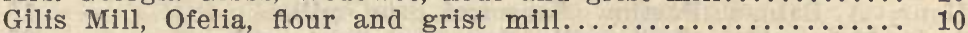

Eppie M. White, Bernice, flour and grist mill........... 5

Larkin \& M. B. Taylor, Lamar, flour and grist mill.......... 8

Joseph B. Taylor. Roanoke, flour and grist mill........... 24

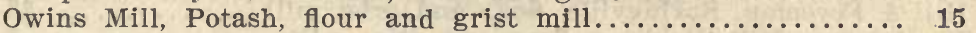

Rogers Mill, Ofelia, flour and grist mill................. 8

C. A. Prescott, Wedowee, flour and grist mill............ 20

H. A. Merrill, Lamar, flour and grist mill............... 6

Elizabeth H. Merrill, Micaville, flour and grist mill.......... 12

J. E. McCosh \& Co., Lime, flour and grist mill........... 40

William S. McCarley, Graham, flour and grist mill........ 20

John $H$. Landers, Lofty, flour and grist mill............. 8

Edward Lavoorn, — flour and grist mill............ 8

Thomas J. Lavoorn, Hawk, flour and grist mill........... 16

Thomas J. Lavoorn, Sr., Newell, flour and grist mill......... 8

James L. \& John T. Kaylor, Kaylor, flour and grist mill...... 60

Henry C. Jordon, Clack, flour and grist mill............. 6

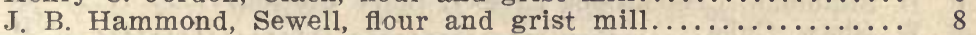

T. M. Halaway, Tolbut, flour and grist mill............. 15

Robert $H$. Harris, Louina, flour and grist mill........... 15

Dock Huckaby. Almond, flour and grist mill............... 10

Holley's Mill, Rock Mills, flour and grist mill.............. 30

E. C. Heaton, Hawk, flour and grist mill............... 10

William N. Gladney, Roanoke, flour and grist mill.......... 12

A. B. East, Christiana, flour and grist mlll.............

Adamson \& Edward's Mills, Ofelia, flour and grist mill ....... 25

Bailey Mill, Haywood, flour and grist mill.............. 12

F. P. Parker, Foresters Chapel, flour and grist mill.......... 10

John C. Murphy, Gay, flour and grist mill............... 2

E. L. Pool, Happyland, flour and grist mill............. 20

James.M. Kitchens, Rockdale, flour and grist mill.......... 8

James H. Wright, Jeptha, flour and grist mill............ 12

Adamson \& Edwards, Ofelia, lumber and timber.......... 40

William W. Brooks, Lofty, lumber and timber............. 15

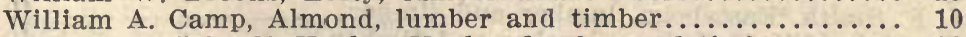

James L. \& John T. Kaylor, Kaylor, lumber and timber...... 20

H. H. Stephens, Pencil, lumber and timber.............. 20

Samuel H. Striplin, Roanoke, leather, tanned, curried \& finished 6

Wehadkee Cotton Mills, Rock Mills, cotton goods........... 108

*From U. S. Census, 1900. 


\section{RUSSELL COUNTY.}

NAME. POSTOFFICE. IXDUSTRY.

( Davis' Mill, Crawford, flour and grist mill.............. 20

$\{$ H. R. Dudley, Seale, lumber and timber..................... 40 $\dagger$ E. M. Anderson, (Watermelon Cr.), Seale, grist mill and gin 20

\section{*SHELBY COUNTY.}

W. C. Denson, Pelham, flour and grist mill...............12 William H. Shrader, Shelby, flour and grist mill.............. 20 William H. Pledger, Pelham, flour and grist mill............... 40 Hendrick \& Alverson, Vincent, flour and grist mill......... 40 David A. Whitfield, Vandiver, flour and grist mill.......... 10 Brownings Mill, Columbiana, lumber and timber............ 30

\section{*ST. CLAIR COUNTY.}

The Yarbrough Mill, Ashville, flour and grist mill........ 8 Hare's Mill, Ashville, flour and grist mill............... 8 John R. Dyke, Wolfcreek, flour and grist mill............. 30 Perry E. Wyatt, Coal City, flour and grist mill.............. 10 Henry A. Palmer, Partlow, flour and grist mill............ 10 J. M. McLaughlin, Springville, flour and grist mill......... 25 The Machen Mill, Partlow, flour and grist mill.............. 10 The Lindsey Mill, Ashville, flour and grist mill............. 10 Hill \& Foreman, Springville, flour and grist mill.......... 28 Henderson's Mill, Ragland, flour and grist mill.............. 5 Helm \& Truss, Helms, flour and grist mill............... 20 Grout's Mills, Wolfereek, flour and grist mill........... 10 The Gilchrist Mill, Ashville, flour and grist mill.......... 5 The Cox Mill, Ashville, flour and grist mill............. 10 Rufus W. Beason, Whitney, flour and grist mill........... 11 Rock Bridge Mill, Gallant, lumber and timber mill........... 20

\section{*SUMTER COUNTY.}

E. B. Hearn, (Kinterbish Creek), Gaston, ............... 40

R. H. Stephens, (Kinterbish Creek), Alamuchee........... 20

R. D. Simmons, (Toomsooba Creek), Bell's Station.......... 30

R. W. Shaw, Cuba ........................... 10

W. H. Walker, (Silver Creek). Alamuchee............. 20

J. U. Gillespie, (Coatopa Creek), Coatopa .............. 10

\section{TALLADEGA COUNTY.}

「 Jefferson Roberson, Fayetteville, flour and grist mill........ 10

J. C. Brock, Eastaboga, flour and grist mill............. 12

Riser \& Bro., Talladega, flour and grist mill............. 40

Shock E. Jemison, Sunnyside, flour and grist mill.......... 15

Vincent Mill, Talladega, flour and grist mill.............. 25

O. F. Luttrell, Talladega, flour and grist mill............... 40

Riddle Mills, Waldo, flour and grist mill............... 16

\{ J. F. Smith, Eastaboga, flour and grist mill............... 40

John W. Thweatt, McFall, flour and grist mill............ 12

J. B. Turner, McFall, flour and grist mill............... 15

Allison's Mill, Talladega, flour and grist mill............ 60

J. F. Smith, Eastaboga, lumber and timber.............. 40

Cragdale Mill, Talladega, lumber and timber.............. 40

J. B. Turner, McFall, lumber and timber............... 20

*From U. S. Census, 1900.

$\nmid$ From report of Probate Judge. 
NAME.

POSTOFFICE.

INDUSTRY.

H. P.

(Priebes Mill, (Choccolocco Creek), Jenifer, grist mill........ 200

J. F. Smith's Mill, (Choccolocco Creek), Oxford, grist mill.... 225

B. Schmidt's Mill, (Choccolocco Creek), Lincoln, grist mill... 200

Craig's Mill, (Choccolocoo Creek), Oxford, grist mill......... 150

Wilson's Mill, (Choccolocco Creek), Jenifer, grist mill....... 150

Eureka Mills, (Choccolocco Creek), Eureka, grist mill........ 150

Turner's Mill, (Chehawhaw Creek), McFall, grist mill....... 150

Kants Mill, (Talladega Creek), Chandler wprings, grist mill.. 50

Riddle's Mill, (Talladega Creek), Waldo, grist mill......... 75

Taylor's Mill, (Talladega Creek), Talladega, grist mill...... 150

Reynold's Mill, (Talladega Creek), Nottingham, grist mill..... 150

Allison's Mill, (Talladega Creek), Talladega, grist mill....... 75

Duncan's Mill, (Talladega Creek), Alpine, grist mill........ 75

Baker's Mill, (Talladega Creek), Kymulga, grist mill........ 100

Vincent's Mill, ,Crooked Creek), Sylacauga, grist mill....... 50

Oden's Mill, (Short Creek), Sylacauga, grist mill.......... 75

Jemison's Mill, (Kelley's Creek), Sunnyside, grist mill....... 50

Camp \& Sons' Mill, (Salt Creek), Hopeful, grist mill......... 50

Robinson's Mill, (Cedar Creek), Fayetteville, grist mill....... 50

Lackey's Mill, (Horse Creek), Ironaton. grist mill .......... 25

Talladega Company, (Choccolocco Creek), Talladega, organized

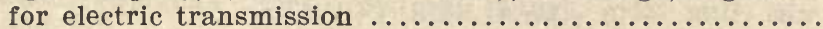

\section{*TALLAPOOSA COUNTY.}

George Stewart, Thaddeus, flour and grist mill........... 12

John W. Britt, Jacksons Gap, flour and grist mill......... 20

Benjamin F. Jarvis, Yates, flour and grist mill........... 12

T. J. Hamlet, Hamlet, flour and grist mill................ 15

T. W. Whitman, Dadeville, flour and grist mill.......... 20

Sanford Milling \& Mfణ. Co., Dadeville, flour and grist mill...25

John W. Hay, Camphill, flour and grist mill............ 15

Hammond's Mill, Dadeville, flour and grist mill.......... 20

Hodnett Grist \& Flour Mill. Acme, flour and grist mill........ 16

Thomas L. Bulger, Dadeville, flour and grist mill.......... 15

Vines Mills, Easton, flour and grist mill.............. 40

A. T. \& H. C. Vickers, Newsite, flour and grist mill........ 20

J. C. Street, Anniston, flour and grist mill.............. 25

Shephard Bros. \& Co., Tohopeka, flour and grist mill......... 10

G. W. Stewart, Thaddeus, flour and grist mill........... 25

Albert J. Hollaway, Alexander City, flour and grist mill..... 20

Mrs. Milliner, Mary, flour and grist mill.............. 25

Jno. L. Patterson, Hackneyville, flour and grist mill.......... 12

Thomas B. Griffin, Matilda, flour and grist mill........... 10

Daviston Mill, Daviston, flour and grist mill............ 8

Lamberth \& Dewberry, Logpit, flour and grist mill........ 20

Silver Shoals Mill, Buttston ,flour and gris mill.......... 80

M. R. Hays \& Bro., Notasulga, flour and grist mill.......... 40

Farrows Flour \& Grist Mill, Susanna, flour and grist mill..... 60

J. H. Yarbrough, Hackneyville. flour and grist mill......... 12

T. F. Garnett, Tallassee, lumber and timber............ 20

G. W. Stewart, Thaddeus, lumber and timber........... 20

*From U. S. Census, 1900.

†From report of Probate Judge. 


\section{*TUSCALOOSA COUNTY.}

NAME.

POSTOFFICE.

INDUSTRY.

H. $\mathbf{P}$.

Price's Mill, Binion, flour and grist mill............... 8

Keene's Mill, Cottondale, flour and grist mill................. 20

B. E. Thompson, Cottondale, flour and grist mili ............. 15

Wm. D. Shadix, (Sandy Creek), Double Springs, flour and grist 4

J. W. Spencer, Elrod, flour and grist mill............... 10

Webb's Mill, Elrod, flour and grist mill................ 20

Hagler's Mill, Falls, flour and grist mill................. 40

Patton's Mill, Fosters, flour and grist mill................. 12

David M. Montgomery, Moores Bridge, flour and grist mill.... 10

Looney John Mills, New Lexington, flour and grist mill...... 6

Eitson's Mill, New Lexington, flour and grist mill........... 12

Alfred Gilliland, Newtonvile, flour and grist mill............ 12

Andrew J. Hewett, Skelton, flour and grist mill............ 5

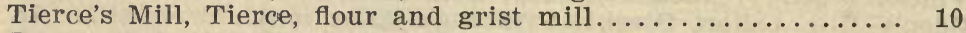

James M. Yerby, Tuscaloosa, flour and grist mill........... 20

O. W. Glenn, Tyner, flour and grist mill............... 8

The Rope \& Yarn Mills, (Binion's Creek), Samantha, cordage

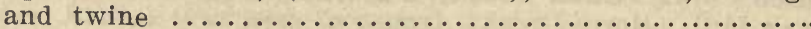

\section{*WALKER COUNTY.}

Boldo Grist Mill, Boldo, flour and grist mill............ 40

James B. Wakefield, Prospect, flour and grist mill.......... 10

Lewis W. Odom, Oakman, flour and grist mill............. 10

Mahala E. \& Dalton Odom, Parrish, flour and grist mill...... 10

Joseph Z. Norris, Galloway, flour and grist mill........... 5

Thomas J. King, Oakman, flour and grist mill............ 10

Lewis Guthrie, Pocahontas, flour and grist mill........... 10

Wm. Cobb, Oakman, flour and grist mill................ 10

Peter McGough, Carbonhill, lumber and timber........... 10

*WASHINGTON COUNTY.

Mrs. Samuel Wilkins, Healing Springs, flour and grist mill.....

Consey's Mill, Healing Springs, lumber and timber mill.......

WILCOX COUNTY.

(Ward \& Grimes, (Pine Barren Creek), Pineapple, flour and grist

* \{ George A. Barge, Snowhill, flour and grist mill.............

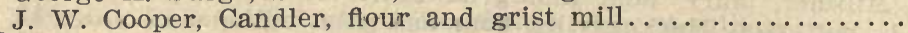

George Barge, (Pine Barren Creek), Furman, grist mill and gin

Glover \& Carter, (Pine Barren Cr.), Pineapple, grist mill and gin S. McCormick, (Pine Barren Cr.), Pineapple, grist mill and gin D. McIntosh, (Pursley Creek), Camden, grist mill and gin....

*From U. S. Census, 1900.

$\dagger$ From report of Probate Judge. 


\section{WINSTON COUNTY.}

NAME

POSTOFFICE.

INDUSTRY.

H. $\mathbf{P}$.

(Richard H. Blake, Houston, flour and grist mill.......... 8

Thomas 0 . Partridge, Elk, flour and grist mill............... 10

Wm. D. Shadix, (Sandy Creek), Double Springs), flour and grist 4

George D. Wilson, Haleysville, flour and grist mill......... 8

- Manna A. Posey, Motes, flour and grist $\backslash$ mill................. 10

Martin A. \& Martha reak, Peaks Mill, flour and grist mill...... 10

Miligan Mill, Double Springs, flour and grist mill.......... 10

James Cantrell, Addison, flour and grist mill.............. 4

Burks Mill, Cranal, flour and grist mill................... 10

( Nauvoo Mill, (Black Water Creek), Nauvoo, grist mill and gìn Anderson Ward Mill, (Clear Creek), Haleysville, flour and grist J. Calvin Cagle, (Clear Creek), Double Springs, saw, flour and

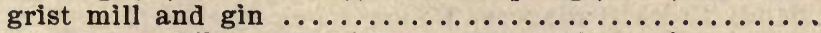
Jonathan Barton Mill, (Clear Creek), Deer, grist mill .......... Hadder Mill, (Clear Creek), Double Springs, grist mill.......... Posey Mill, (Clear Creek), Motes, grist mill, saw and gin...... S. D. Spain, (Clear Creek), Malta, grist mill, saw and gin..... Gus Posey Mill, (Clear Creek), Elk, grist mill, saw and gin.... Wm. Dodd, (Splunge Creek), Natural Bridge, grist mill, saw

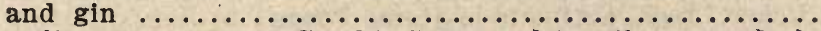
Kelley Mill, (Black Water Creek), Lynn, grist mill, saw and gin Peaks Mill, (Grindstone Creek), Peaks Mill, grist mill, saw and

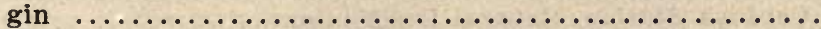
Jack Curtis, (Sandy Creek), Double Springs, grist mill, saw and

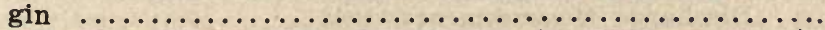
Manley Payne, (Beech Creek), Gumpond, grist mill, saw and gin (Christian Mill, (Christian Creek), Peaks Mill, grist mill, saw

*From U. S. Census, 1900.

†From report of Probate Judge. 


\section{INDEX.}

Alabama River at Selma:

General Description of Station ................. 85

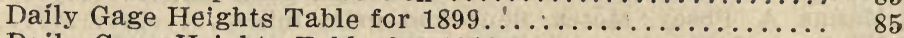

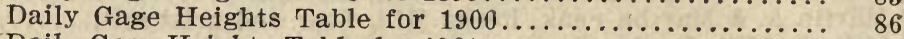

Daily Gage Heights Table for $1901 \ldots \ldots \ldots \ldots \ldots \ldots \ldots \ldots \ldots \ldots \ldots . .6 \%$

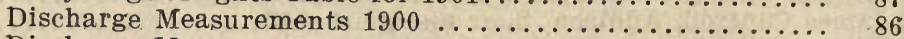

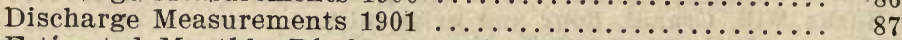

Estimated Monthly Discharge, $1900-1901 \ldots \ldots \ldots \ldots \ldots \ldots . . . . . .69$

Minimum Monthly Discharge and Horse Power, 1899-1901.. 91

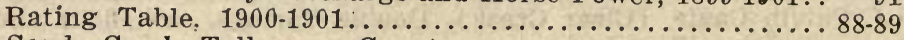

Big Sandy Creek, Tallapoosa County:

Daily Gage Heights, $1900-1901 \ldots \ldots \ldots \ldots \ldots \ldots \ldots \ldots \ldots . . \ldots \ldots$

Discharge Measurements, $1900 \ldots \ldots \ldots \ldots \ldots \ldots \ldots \ldots \ldots . \ldots \ldots$

Elevations and Bench Marks along .................... 45

Minimum' Monthly Discharge and Horse Power, 1900-1901. 43

Profile ................................ 44

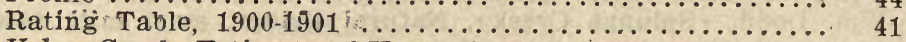

Big Uchee Creek, Estimate of Horse Power................ 11

Big Wills Creek, Wesson's Mill, Discharge Measurements...... 91

Black Warrior River:

Cordova Station, General Descrintion ............. 120

Daily Gage Heights, $1900-1901$................. 120-121

Discharge Measurements, $1901 \ldots \ldots \ldots \ldots \ldots \ldots \ldots \ldots . \ldots \ldots \ldots . \ldots \ldots 121$

Estimated Monthly Discharge Mulberry Fork, 1900-1901 123

Minimum Monthly Discharge and H. P., 1900-1901..... 124

Rating Taoles, $1900-1901 \ldots \ldots \ldots \ldots \ldots \ldots \ldots \ldots \ldots, 122$

Tuscaloosa Station, General Description .............. in $4^{2}$

Daily Gage Heights, $1889-1901 \ldots \ldots \ldots \ldots \ldots \ldots \ldots \ldots \ldots \ldots \ldots \ldots \ldots \ldots .113$

Discharge Measurements, 1895-1901.......105, 110, 111. 113

Discharge (Graphíc), 1889-1899...............118-119

Estimated Monthly Discharge, $1895-1900 \ldots \ldots 116,117,118$

Minimum Monthly Discharge and H. P., 1899-1901... 119

Rating Table, 1895-1901 ......................... 115

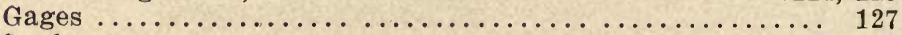

Locks ...............................125, 126, 127

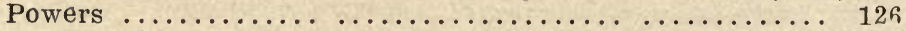

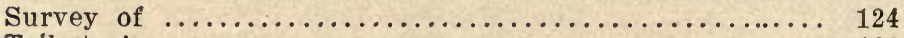

Tributaries .................................. 198

Clear Creek ......................... 128

Little Warrior or Locust Fork ............. 128

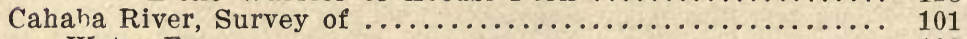

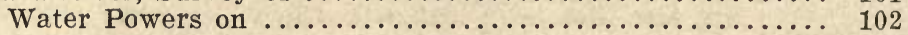

Centerville Station. General Description ............. 99

Daily Gage Heights, $1901 \ldots \ldots \ldots \ldots \ldots \ldots \ldots \ldots \ldots . \ldots \ldots$

Discharge Measurements, $1901 \ldots \ldots \ldots \ldots \ldots \ldots \ldots . . . . . .69$

Minimum Monthly Discharge and H. P., $1901 \ldots \ldots \ldots, 101$

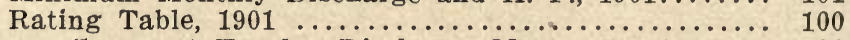

Choccolocco Creek, at Eureka, Discharge Measurements..... 91

Near Juniper ............................ 91 
INDEX.

Coosa River:

Riverside, Ala., Station, General Description......... 53 Daily Gage Heights, $1896-1901 \ldots \ldots \ldots .54,56,58,60,62,63$ Discharge Measurements, $1896-1901 \ldots \ldots 54,55,57,60,62,63$ Estimated Monthly Discharge, 1896-1901.........65, 66 Graphic Illustration of Discharge, $1897-1899 \ldots \ldots \ldots 67,68$ Minimum Monthly Discharge and H. P., 1899-1901... 68

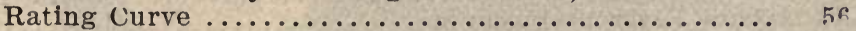
Rating Table, $1896-1901 \ldots \ldots \ldots \ldots \ldots \ldots .55,57,59,61,64$

Rome, Ga., Station, General Description ............ 69 Daily Gage Heights $1897-1901 \ldots \ldots \ldots \ldots 70,71,73,76,77$ Discharge Measurements, $1896-1901 \ldots \ldots .69,70,72,75,76$ Estimated Monthly Discharge $1897-1901 \ldots \ldots \ldots \ldots .78,79$ Graphic Illustration of Discharge, $1897-1899 \ldots \ldots \ldots .80 .81$ Minimum Monthly Discharge and H. P., 1899-1901... 81 Rating Table, $1897-1901 \ldots \ldots \ldots \ldots \ldots \ldots \ldots \ldots 72,74,75,77$

Survey of. above Wetumpka .................. 93

Table of Discharge and Net H. P. at Lock Sites on....... 95

Tributaries of above Wetumpka ................ 92

Water Powers on Tributaries .................. 92-93

Cowikee Creek, Estimate of Horse Power.............. 11

Discharge Measurements-Miscellaneous:

Big Wills Creek, Wesson's Mill ..................,91

Choccolocco Creek at Eureka and near Juniper.......... 91

Hatchet Creek at Goodwater ................... 91

Talladega Creek at Kymulga .................... 91

Tallasseehatchee Creek at Childersburg............. 91

Lstimates of Horse Powers-Miscellaneous:

Big Uchee Creek ............................. 11

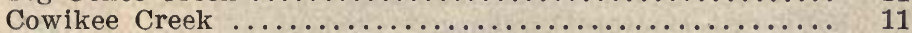

Hatchechubbee Creek ........................ 11

Ihagee Creek ............................. 11

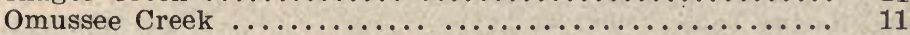

Yattayabba Creek ........................... 11

Estimated Monthly D.scharge, Explanations of ........... 13

Gage Heights. Explanation of ..................... 11

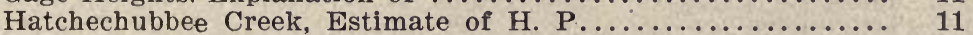

Hatchet Creek at Goodwater, Discharge Measurement....... 91

Hillabee Creek:

Description of Station $\ldots \ldots \ldots \ldots \ldots \ldots \ldots \ldots \ldots \ldots \ldots \ldots$

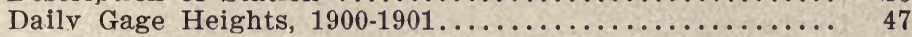

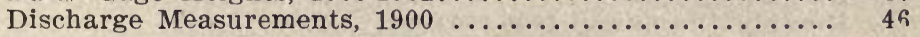

Estimated Monthly Discharge, $1900-1901 \ldots \ldots \ldots \ldots \ldots \ldots .49$

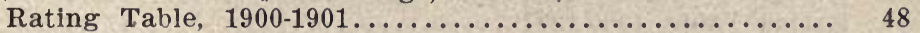

Ihagee Creek, Estimate of H. P................. 11

Omussee Creek, Estimate of H. P.................. 11

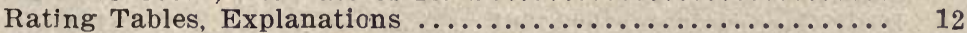

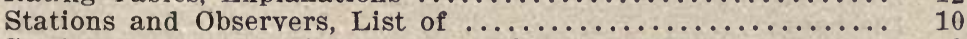

Stations and Water Powers, General Statements........... 9

Talladega Creek at Kymulga, Discharge Measurement....... 81

Talladega Creek at Nottingham ...................... 82

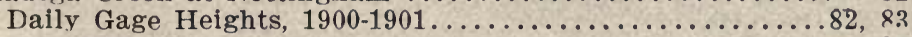

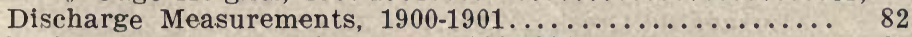

Estimated Monthly Discharge, $1900-1901 \ldots \ldots \ldots \ldots \ldots \ldots . \ldots . \ldots$

Minimum Monthly Discharge and H. P., 1900-1901....... 84

Rating Table, $1900-1901 \ldots \ldots \ldots \ldots \ldots \ldots \ldots \ldots \ldots \ldots \ldots \ldots \ldots \ldots$ 
Tallapoosa River at Milstead ..................... 15

Daily Gage Heights $\ldots \ldots \ldots \ldots \ldots \ldots \ldots \ldots \ldots 16,17,19,21,22$

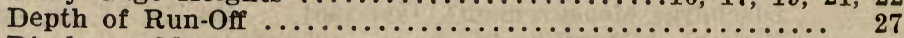

Discharge Measurements ................. 15, 18, 20, 21

Minimum Monthly Discharge ................. 27

Rating Table ............................ $18,20,23$

Tallapoosa River at Susanna ........................ 28

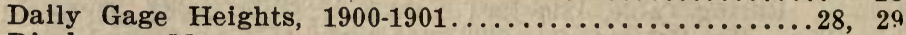

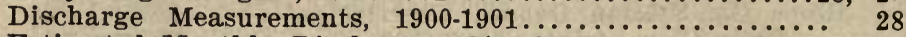

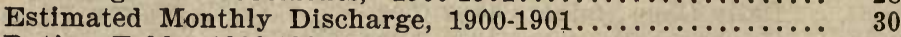

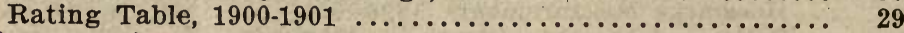

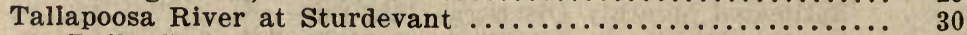

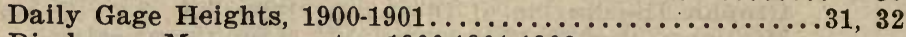

Discharge Measurements, $1900-1901-1902 \ldots \ldots \ldots \ldots \ldots \ldots \ldots . \ldots \ldots \ldots . \ldots \ldots$

Tallapoosa River, Elevations and Bench Marks along between

Millstead and Griffin Stations ............... 34

Tallapoosa River, Map of Milstead to Griffin Shoals, Fig. $4 \ldots \ldots$.... 37

Tallapoosa River, Prafile of Griffin Shoals to Milstead....... 38

Survey of in Alabama ........................ 32

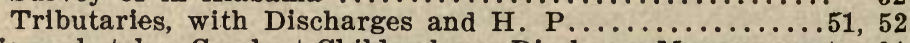

Tallasseehatchee Creek at Childersburg, Discharge Measurement 91

Tennessee River at Chattanooga . ................... 139

General Description ....................... 139

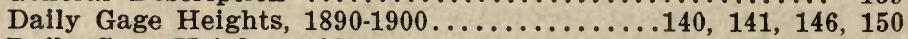

Daily Gage Helghts, $1901 \ldots \ldots \ldots \ldots \ldots \ldots \ldots \ldots \ldots \ldots \ldots$

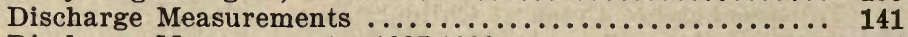

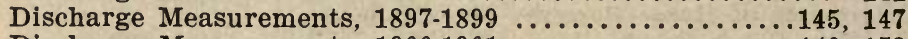

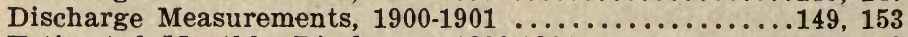

Estimated Monthly Discharge, $1890-1901 \ldots \ldots \ldots \ldots \ldots .156,159$

Estlmated Monthly Discharge (Graphic), 1891-1898...... 160

Minimum Monthly Discharge, $1899-1901 \ldots \ldots \ldots \ldots \ldots \ldots 1$

Rating Table, $1890-1900 \ldots \ldots \ldots \ldots \ldots \ldots \ldots$. $\ldots \ldots \ldots, 148,151,152$

Tennessee River, Shoals near Florence ................ 161

Estimated Minlmum H. P.................... 163

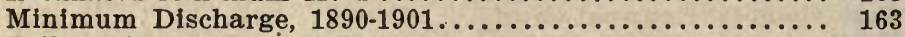

Tributaries ................................. 164

Tombigbee River at Columbus, Miss ................. 129

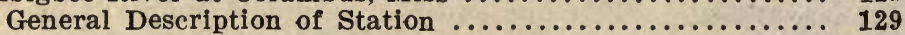

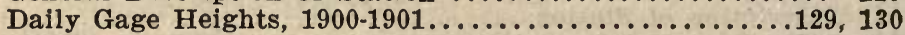

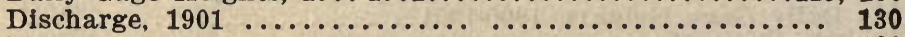

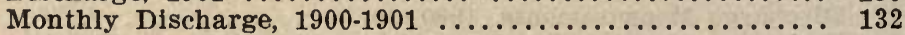

Minimum Monthly Discharge, $1900-1901 \ldots \ldots \ldots \ldots \ldots \ldots . \ldots 133$

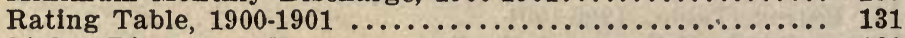

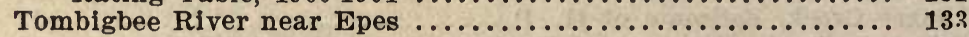

General Description ......................... 133

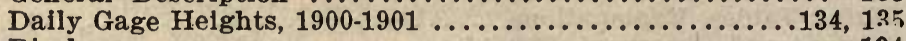

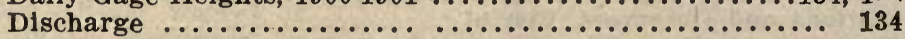

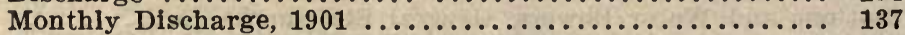

Minimum Monthly Discharge, $1900-1901 \ldots \ldots \ldots \ldots \ldots \ldots . \ldots \ldots \ldots \ldots$

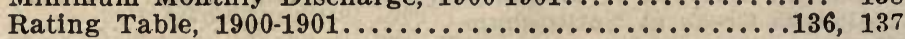

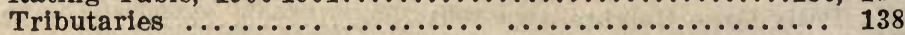

Luxapalila Creek ................................. 138

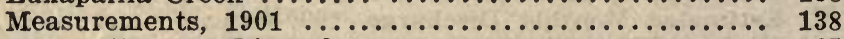

Utilized Water Powers, List of ................... 165

Yattayabba Creek, Estimated H. P. of ............... 11 





\section{DAY USE}

RETURN TO DESK FROM WHICH BORROWED

EARTH SCIENCES LIBRARY

This book is due on the last date stamped below, or

on the date to which renewed.

Renewed books are subject to immediate recall.

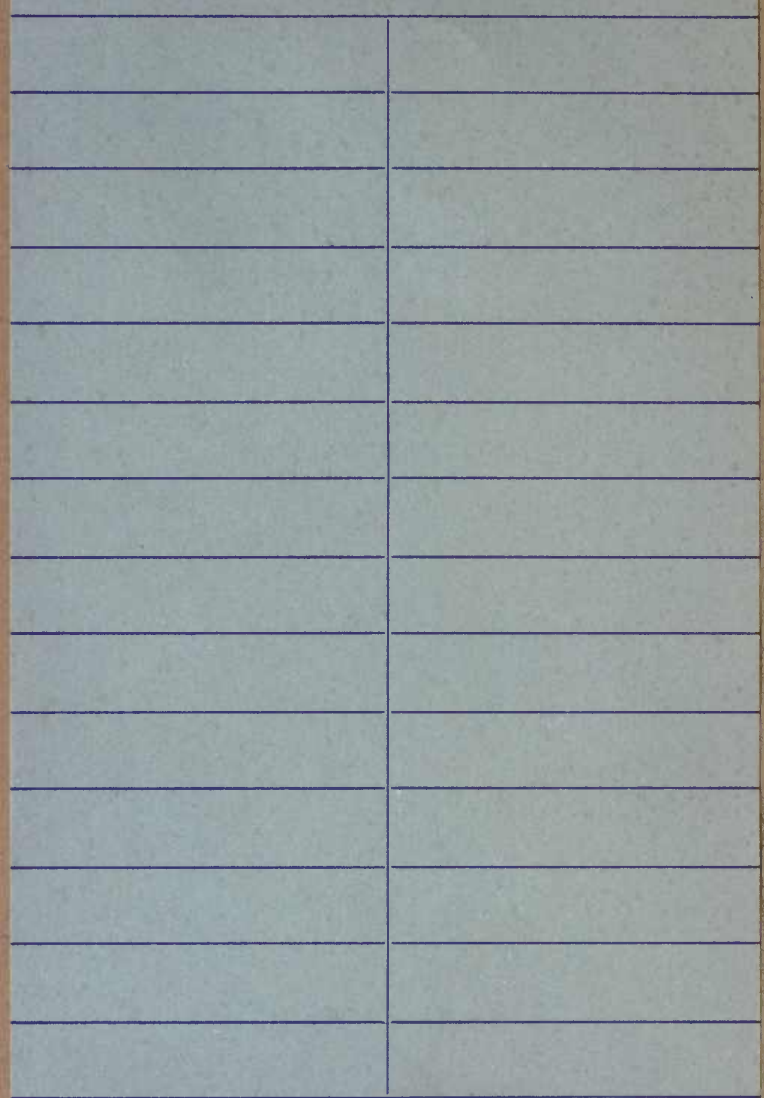

LD 21-40m-5,'65

(F4308s10) 476

General Library

University of California Berkeley 
857

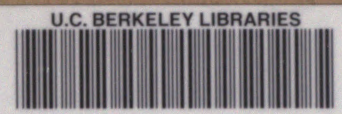
C0335b2725 
